\title{
Critical issues for predicting worker exposure to gaseous contaminants in a wind tunnel
}

Jun Li

West Virginia University

Follow this and additional works at: https://researchrepository.wvu.edu/etd

\section{Recommended Citation}

$\mathrm{Li}$, Jun, "Critical issues for predicting worker exposure to gaseous contaminants in a wind tunnel" (2005). Graduate Theses, Dissertations, and Problem Reports. 2665.

https://researchrepository.wvu.edu/etd/2665

This Dissertation is protected by copyright and/or related rights. It has been brought to you by the The Research Repository @ WVU with permission from the rights-holder(s). You are free to use this Dissertation in any way that is permitted by the copyright and related rights legislation that applies to your use. For other uses you must obtain permission from the rights-holder(s) directly, unless additional rights are indicated by a Creative Commons license in the record and/ or on the work itself. This Dissertation has been accepted for inclusion in WVU Graduate Theses, Dissertations, and Problem Reports collection by an authorized administrator of The Research Repository @ WVU.

For more information, please contact researchrepository@mail.wvu.edu. 


\title{
Critical Issues for Predicting Worker Exposure to Gaseous Contaminants in a Wind Tunnel
}

\author{
Jun Li \\ Dissertation submitted to the \\ College of Engineering and Mineral Resources \\ at West Virginia University \\ in partial fulfillment of the requirements \\ for the degree of \\ Doctor of Philosophy \\ in \\ Mechanical Engineering \\ Ismail Celik, Ph.D., Chair \\ Nigel Clark, Ph.D. \\ John Kuhlman, Ph.D. \\ Steve Guffey, Ph.D. \\ Ibrahim Yavuz, Ph.D., Co-chair
}

Mechanical and Aerospace Engineering Department

Morgantown, West Virginia

2005

Keywords: Worker exposure assessment, Reynolds-averaged Navier-Stokes equations, Non-linear turbulence model

Copyright 2005 Jun Li 


\section{ABSTRACT}

\section{Critical Issues for Predicting Worker Exposure to Gaseous Contaminants in a Wind Tunnel \\ Jun Li}

In this study, three dimensional computational fluid dynamics (CFD) simulations are used to investigate the distribution and level of contaminant concentrations in the breathing zone of a worker when airborne contaminants are released within an arm's-length in front of the worker who has his back to the airflow. The main goals were to numerically evaluate the effect of different factors on the worker exposure and to recommend a turbulence model preferable for this type of simulation. These factors include the body shape, the heat flux from the body, the ventilation intensity, the free stream turbulence, and the unsteadiness. The comparison between the numerical results and the experimental data has shown good agreement.

An extensive case study with FLUENT concluded with the following observations: 1) The heat flux from the body significantly affects the flow field and the subsequent contaminant concentration field at low Reynolds numbers; 2) The free stream turbulence plays an important role in the variation of exposure measurements at low Reynolds numbers; 3) Results calculated with the Large Eddy Simulation (LES) illustrate the turbulence structure in the wake of the manikin and indicate that the flow unsteadiness plays an important role in the variation of exposure measurements; 4) Calculations with various body shapes suggests that oversimplified body shapes may lead to inaccurate predictions in worker exposure assessment; 5) The concentrations measured at the lapel could be very different than the concentrations measured near the mouth.

To further improve the predictability of turbulence models for the present study, a non-linear (cubic) low-Re turbulence model has been selected, modified and implemented in the DREAM code which was developed at West Virginia University. Benchmark tests on turbulent channel flow, backward facing step flow and flow around a square cylinder have shown that this model is remarkably superior to linear eddy-viscosity models, and the results are even comparable to others' predictions with LES, which is much more computationally expensive. So it could be a good alternative as a reliable and accurate turbulence model in simulating turbulent flow past a bluff body. 


\section{ACKNOWLEDGMENTS}

I would like to give my profound thanks to my advisor and committee chair, Dr. Ismail Celik, for not only providing the opportunity and challenge to advance my education but more importantly teaching and encouraging me throughout my Ph.D. study. I would also like to thank my committee members, Dr. Nigel Clark, Dr. John Kuhlman, Dr. Steve Guffey and Dr. Ibrahim Yavuz for generously allotting their time, advice and encouragement.

A great number of other people helped in the completion of this study. Special thanks give to my co-advisor, Dr. Ibrahim Yavuz, who has always been there for me when the work was not going well. To Dr. Steve Guffey and his lab, who supported constantly on providing the experimental results. To Dr. Aaron J. Bird, who provided the grid of the human torso. To Dr. Shaoping Shi, who gave me valuable suggestions when I began to use FLUENT, and to the CFD lab, all of whom made my journey at WVU fun and enjoyable.

I will be forever indebted to my parents and parents-in-law for all their support and encouragement. Especially, I am grateful to my wife Chang for the inspiration and moral support she provided throughout my research work. Without her loving support and understanding I would never have completed my present work. Particularly, I owe to my son Frank for the happy moments he brings to us. I dedicate this work to my wife and my son.

This work has been performed under a U.S. National Institute of Occupational Safety and Health (NIOSH) project sponsored by the Center for Disease Control (CDC) under the grant R01 OH07587-011. 


\section{Nomenclature}

\begin{tabular}{|c|c|}
\hline$a_{i j}$ & strain rate \\
\hline $\mathrm{C}$ & mean concentration \\
\hline $\mathrm{C}_{1 \ldots 7}, \mathrm{C}_{\mu}$ & empirical constants in turbulence models \\
\hline $\mathrm{D}$ & dimension of square cylinder \\
\hline $\mathrm{D}_{\mathrm{P}}$ & diameter of the particle \\
\hline $\mathrm{E}$ & near-wall source term in $\varepsilon$ equation of the cubic model \\
\hline $\mathrm{F}_{\mathrm{x}}$ & additional force in particle transport equation \\
\hline f & frequency \\
\hline $\mathrm{f}_{1}, \mathrm{f}_{2}$ & damping functions \\
\hline $\mathrm{G}_{\mathrm{ij}}$ & buoyancy induced production rate of turbulence \\
\hline $\mathrm{Gr}$ & Grashof number \\
\hline$g_{\mathrm{i}}$ & component of the gravitational vector in the ith direction. \\
\hline $\mathrm{H}$ & step height \\
\hline h & enthalpy, mesh size \\
\hline $\mathrm{k}$ & turbulent kinetic energy \\
\hline $\mathrm{L}_{\mathrm{ij}}$ & molecule diffusion \\
\hline $1_{\mu}, 1_{\varepsilon}$ & length scales \\
\hline $\mathrm{P}$ & dynamic pressure \\
\hline $\mathrm{P}_{\mathrm{k}}$ & production rate of turbulent kinetic energy \\
\hline $\mathrm{Pe}$ & Peclet number \\
\hline $\operatorname{Re}$ & Reynolds number \\
\hline $\mathrm{Re}_{\mathrm{P}}$ & particle Reynolds number \\
\hline $\mathrm{R}_{\mathrm{ij}}$ & pressure redistribution \\
\hline Rt & local Reynolds number \\
\hline $\mathrm{Sc}_{\mathrm{L}}$ & laminar Schmidt number \\
\hline $\mathrm{Sc}_{\mathrm{T}}$ & turbulent Schmidt number \\
\hline $\mathrm{S}_{\mathrm{ij}}$ & strain rate \\
\hline $\mathrm{S}_{\mathrm{T}}$ & heat generation source term \\
\hline $\mathrm{St}$ & Strouhal number \\
\hline $\mathrm{S}_{\phi}$ & scalar generation source term \\
\hline $\mathrm{T}$ & temperature \\
\hline $\mathrm{T}_{\mathrm{ij}}$ & turbulent diffusion term \\
\hline $\mathrm{T}_{\text {ref }}$ & reference temperature \\
\hline $\mathrm{t}$ & time \\
\hline $\mathrm{U}$ & mean velocity \\
\hline
\end{tabular}




$\begin{array}{ll}\mathrm{U}_{0} & \text { inflow velocity } \\ \mathrm{U}+ & \text { normalized velocity } \\ \mathrm{u}_{\mathrm{i}}(\mathrm{u}, \mathrm{v}, \mathrm{w}) & \text { Cartesian velocity component in direction } \mathrm{x}_{\mathrm{i}} \\ u^{\prime}, v^{\prime}, w^{\prime} & \text { velocity fluctuation components } \\ \mathrm{u}_{\tau} & \text { friction velocity } \\ \overline{u_{i} u_{j}} & \text { turbulent stress } \\ \mathbf{u}_{\mathrm{P}} & \text { velocity of the particle } \\ \mathrm{V} & \text { inlet velocity for the wind tunnel } \\ \mathrm{x}_{\mathrm{i}} & \text { Cartesian coordinate in tensor notation } \\ \mathrm{y} & \text { normal distance to the wall } \\ \mathrm{y}+ & \text { normalized distance to the wall }\end{array}$




\section{Greek nomenclature}

$\begin{array}{ll}\alpha & \text { grid refinement ratio } \\ \beta & \text { thermal expansion ration } \\ \delta_{\mathrm{ij}} & \text { Kronecker delta } \\ \varepsilon & \text { turbulent dissipation energy } \\ \phi & \text { a scalar } \\ \kappa & \text { von Karman constant } \\ \rho & \text { density } \\ \rho_{0} & \text { density at reference temperature } \\ \rho_{\mathrm{P}} & \text { density of the particle } \\ \sigma_{\mathrm{k}}, \sigma_{\varepsilon} & \text { empirical constants in the standard k- } \varepsilon \text { turbulence model } \\ \sigma_{\mathrm{T}}^{\mathrm{t}} & \text { turbulent Prandtl number } \\ \mu & \text { fluid viscosity } \\ \nu & \text { kinematic viscosity } \\ v_{\mathrm{t}} & \text { turbulent eddy viscosity } \\ \tau & \text { time scale } \\ \Omega_{\mathrm{ij}} & \text { vorticity tensor }\end{array}$




\section{Abbreviations}

$\begin{array}{ll}\text { AES } & \text { approximate error spline } \\ \text { ASM } & \text { algebraic stress model } \\ \text { CD } & \text { central differencing } \\ \text { CFD } & \text { computational fluid dynamics } \\ \text { DES } & \text { detached eddy simulation } \\ \text { DNS } & \text { direct numerical simulation } \\ \text { EVM } & \text { eddy viscosity model } \\ \text { LES } & \text { large eddy simulation } \\ \text { LEVM } & \text { linear eddy viscosity model } \\ \text { QUICK } & \text { quadratic upstream interpolation for convective kinematics } \\ \text { RANS } & \text { Reynolds-averaged Navior-Stokes } \\ \text { RE } & \text { Richardson extrapolation } \\ \text { RSM } & \text { Reynolds stress model } \\ \text { SGS } & \text { subgrid scale } \\ \text { UW } & \text { upwinding }\end{array}$




\section{Table of Contents}

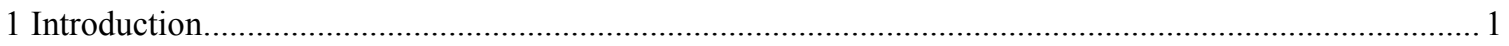

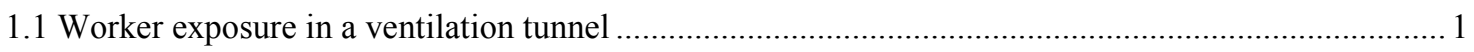

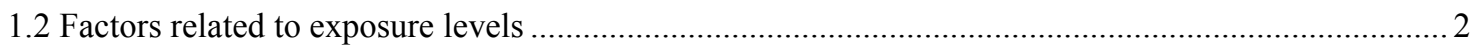

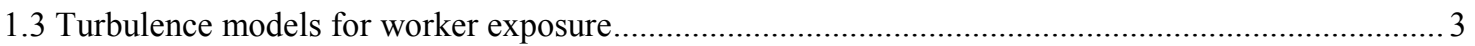

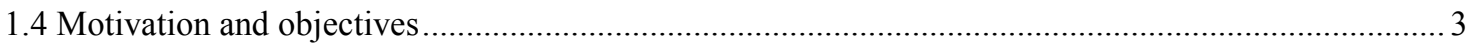

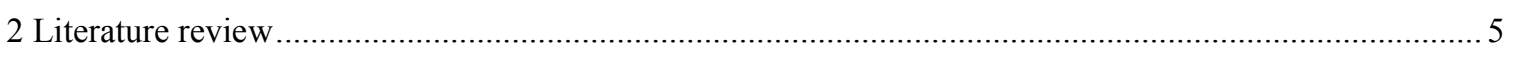

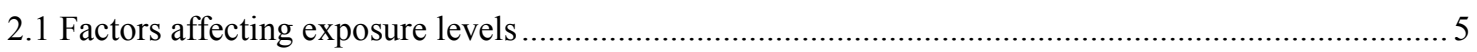

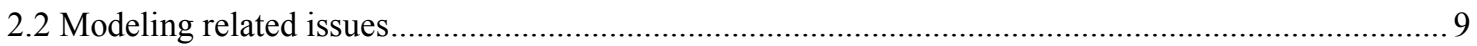

2.2.1 Advanced RANS turbulence models................................................................................ 10

2.2.1.1 Non-linear k- $\varepsilon$ turbulence model...................................................... 11

2.2.1.2 Reynolds stress model (RSM) /Second momentum closure (SMC)..... 12

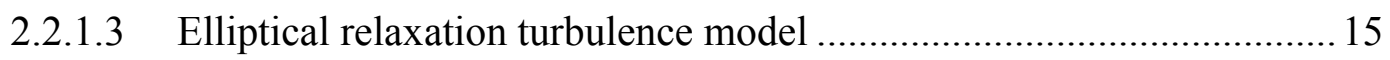

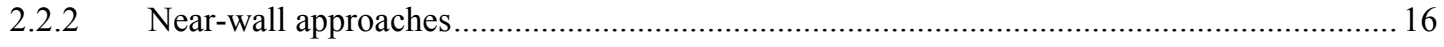

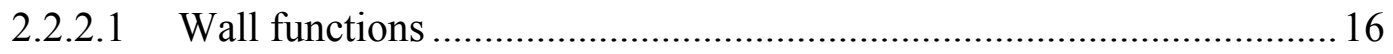

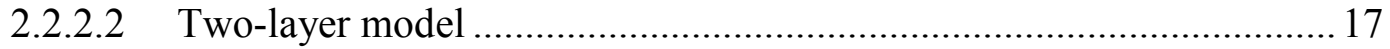

2.2.2.3 Low Reynolds number model .................................................. 18

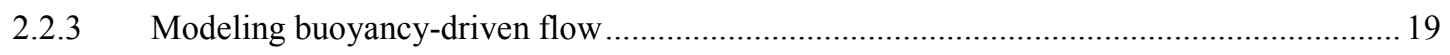

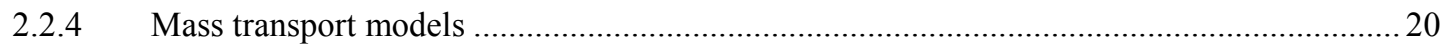

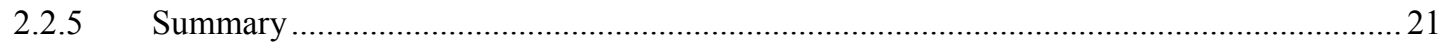

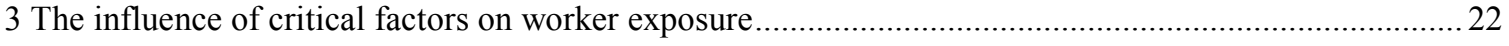

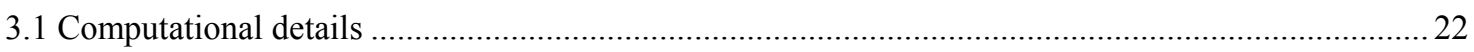

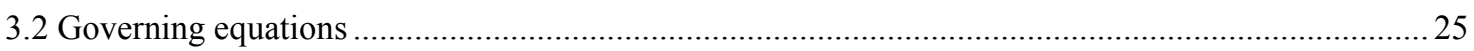

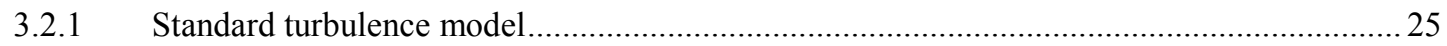

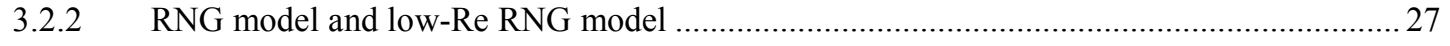

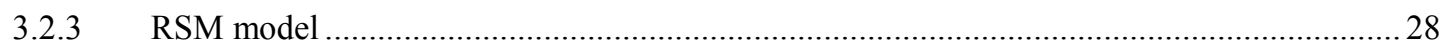

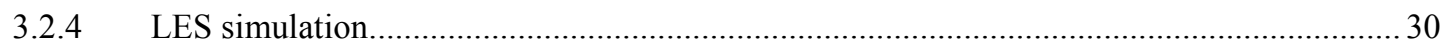

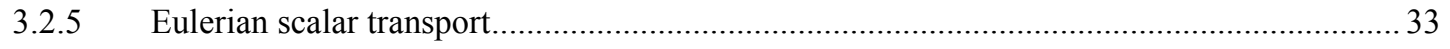

3.2.6 Lagrangian trajectory tracking …………........................................................................ 33

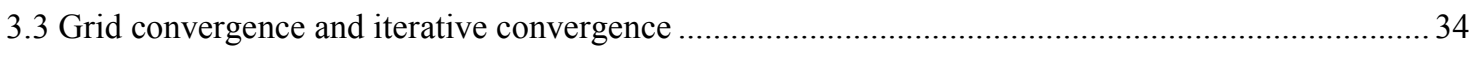

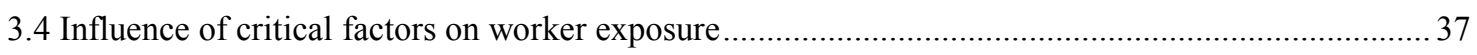

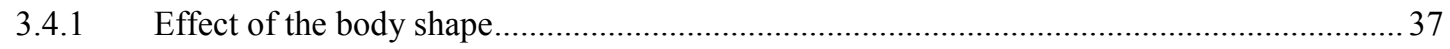

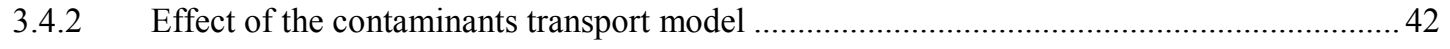

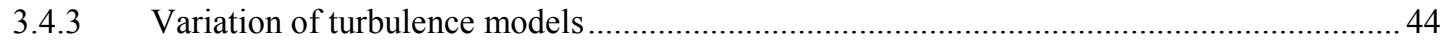

3.4.4 Effect of ventilation intensity, free stream turbulence, and heat flux from the body ............ 49 


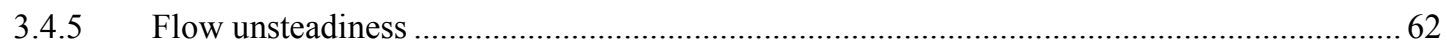

3.5 Summary

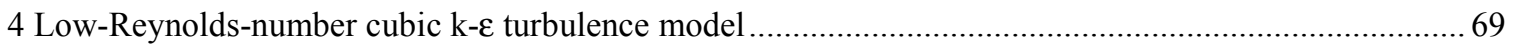

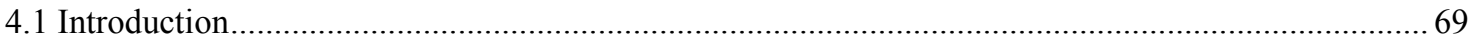

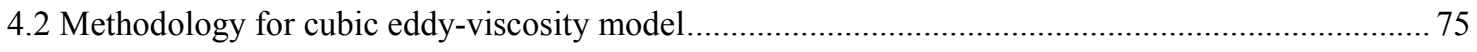

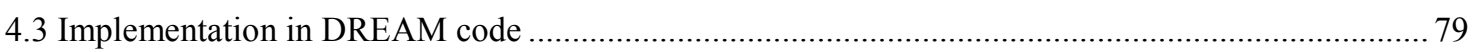

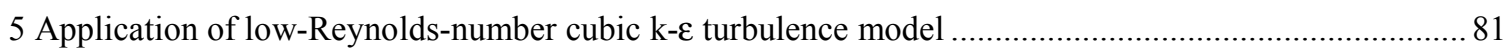

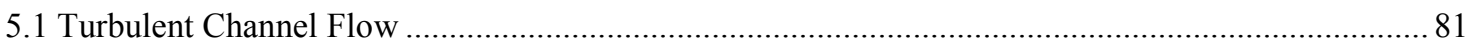

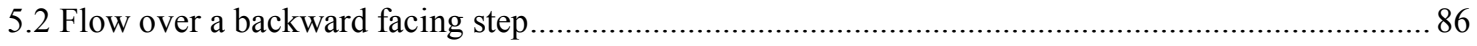

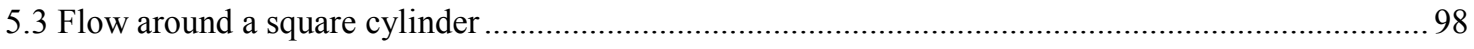

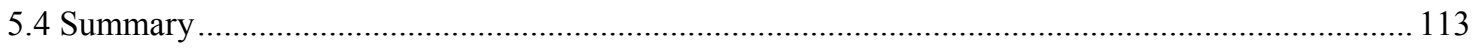

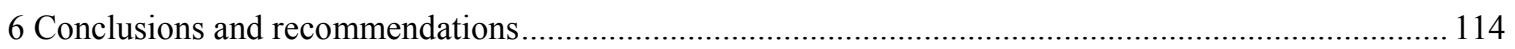

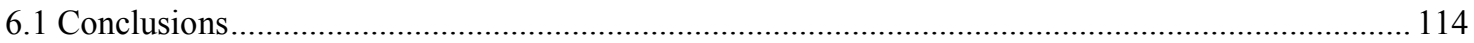

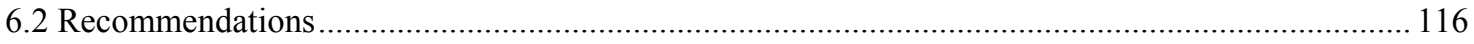

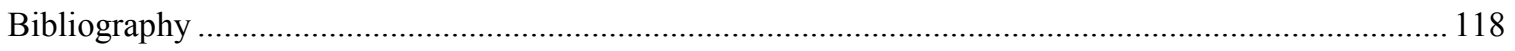

Appendix A. Methods for Numerical Uncertainty Estimation.................................................................. 128

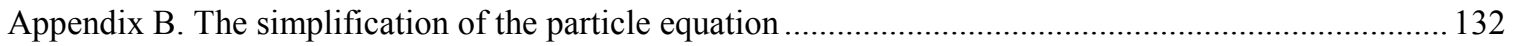

Appendix C. User guide for the cubic turbulence model in DREAM code .............................................. 133 


\section{List of Figures}

Figure 3-1 Schematic view of the wind tunnel model and location of contaminant release plane 23

Figure 3-2 The geometry of the wind tunnel and the human body (all dimensions in $\mathrm{m}$ ) 23 Figure 3-3 Different body shapes used in the present study (not to scale) ...................... 24 Figure 3-4 An example of a computational mesh used in the simulations (Mesh \#2 for the sharp body, 115,824 hexahedral cells; the empty area represents the center of the sharp body).....

Figure 3-5 Streamwise velocities, turbulent kinetic energy, and concentrations at several vertical lines downstream of the body on the central plane with different grid distributions for the sharp body.....

Figure 3-6 Concentrations at the breathing zone with different grid distributions and different body shapes

Figure 3-7 Comparison of the flow field with a block, a sharp body and a rounded body velocity vectors and streamlines on the center plane (side elevation) .......................39

Figure 3-8 Comparison of normalized concentration at different points $1.0 \mathrm{~cm}$ downstream of human body for different body shapes (Normalized with the highest concentration value)

Figure 3-9 Concentration distribution; Eulerian method, on a cross-section $1.0 \mathrm{~cm}$ downstream of the body

Figure 3-10 Concentration contours a,b,c) on the center plane d,e) on a cross section 1.0 $\mathrm{cm}$ downstream of the body

Figure 3-11 Normalized concentrations at different locations at a cross section $1 \mathrm{~cm}$ downstream of the sharp body

Figure 3-12 Streamlines colored by y-velocity in the middle cross section plane with different turbulence models.

Figure 3-13 Tke/subgrid Tke at different horizontal distances from the mouth along a vertical line in the middle plane (Ksgs $=$ Subgrid scale turbulent kinetic energy) ... 47

Figure 3-14 Scalar concentrations $\left(\mathrm{kg} / \mathrm{m}^{3}\right)$ at different horizontal distances from the mouth along a vertical line in the middle plane ....................................................... 48

Figure 3-15 Concentration $\left(\mathrm{kg} / \mathrm{m}^{3}\right)$ levels at different sampling locations with different turbulence models; Eulerian scalar transport 48

Figure 3-16 Schematic view of the computational domain (short domain) .......................50

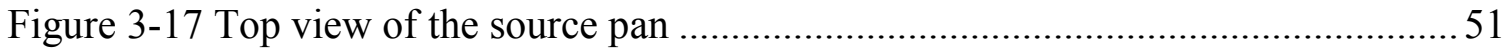

Figure 3-18 The computational mesh in the center plane ………………….................. 51

Figure 3-19 Concentration at the breathing zone vs. iterations at an inlet velocity of 30 fpm $(0.15 \mathrm{~m} / \mathrm{s})$

Figure 3-20 Comparison of the numerical and experimental results of the concentration at

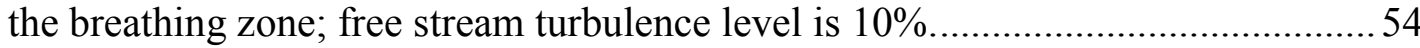

Figure 3-21 The effects of the ventilation intensity and the free stream turbulence on the concentration at the breathing zone (with RNG turbulence model)..........................54

Figure 3-22 The effect of the ventilation intensity and the body heat on the concentration at the breathing zone; $\mathrm{Tu}=10 \%$ .56

Figure 3-23 Heat flux via convection and radiation with RNG turbulence model and averaged temperature boundary condition (B2). 
Figure 3-24 The effect of the ventilation intensity on the concentration at the chest .......57

Figure 3-25 The effect of the ventilation intensity and the body heat on the concentration

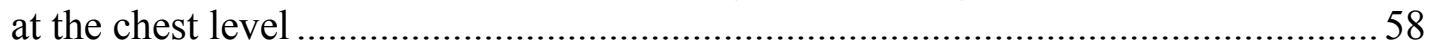

Figure 3-26 Turbulent kinetic energy contours and pathlines in the middle plane at

different ventilation intensity with unheated and heated bodies ........................... 60

Figure 3-27 Concentration contours and pathlines in the middle plane at different ventilation intensity with unheated and heated bodies ....................................... 61

Figure 3-28 The effect of the free stream turbulence with the unheated body at V(inlet) = $10 \mathrm{fpm}$ and $\mathrm{Tu}=60 \%$...

Figure 3-29 Instantaneous Pathlines and concentration contours in the middle plane at $\mathrm{t}=$ $600 \mathrm{~s}$ (concentration :kg/kgair)

Figure 3-30 Instantaneous Pathlines and concentration contours in the horizontal plane $\mathrm{z}$ $=1.51 \mathrm{~m}$ at $\mathrm{t}=600 \mathrm{~s}$ (concentration $: \mathrm{kg} / \mathrm{kgair}$ ).

Figure 3-31 Instantaneous Pathlines and concentration contours in the horizontal plane $\mathrm{z}$ $=1.16 \mathrm{~m}$ at $\mathrm{t}=600 \mathrm{~s}$ (concentration $: \mathrm{kg} / \mathrm{kgair}$ )

Figure 3-32 Instantaneous Pathlines and concentration contours in the middle plane at $\mathrm{t}=$ $620 \mathrm{~s}$ (concentration :kg/kgair)

Figure 3-33 Concentration at the breathing zone changing with time (unheated body; LES)

Figure 3-34 Concentration at the central chest changing with time (unheated body; LES)

Figure 3-35 The power spectrum of concentration at the breathing zone.

Figure 4-1 The variation of $\overline{u_{i}^{\prime} u_{j}^{\prime}} / k$ with strain rate (Craft et al. 1996)............................. 78

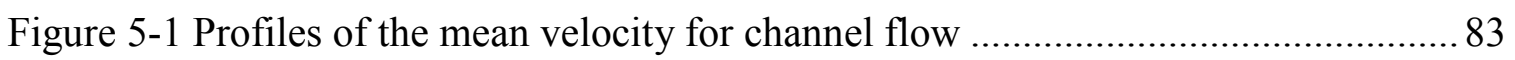

Figure 5-2 Profiles of turbulent normal stresses for channel flow ................................... 84

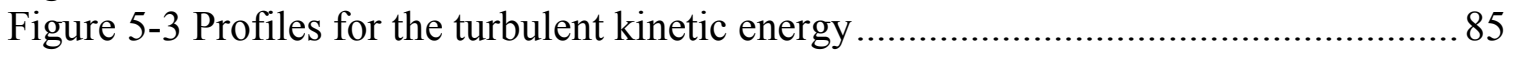

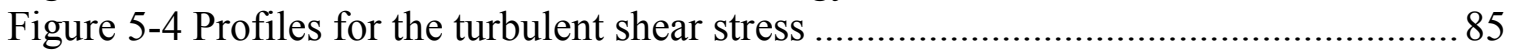

Figure 5-5 The computational domain for the backward facing step flow of (Le and Moin,

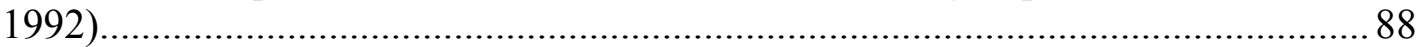

Figure 5-6 Streamfunction contours in and around recirculation zone $(90 \times 150,0.01 \mathrm{~s}) \ldots 88$

Figure 5-7 Grid convergence of the reattachment length with respect to the normalized

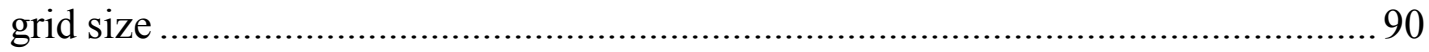

Figure 5-8 Time convergence of the reattachment length with respect to the time step... 90

Figure 5-9 Streamwise velocity profiles (solid line: simulations with 90x150 and $\mathrm{dt}=0.01 \mathrm{~s}$; symbols: DNS by Le \& Moin) ................................................... 91

Figure 5-10 Turbulent kinetic energy profiles (solid line: simulations with 90x150 and

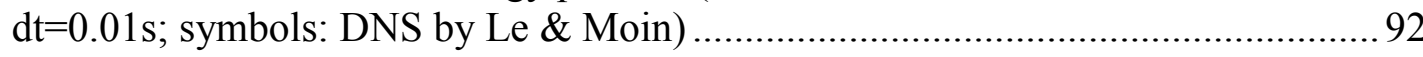

Figure 5-11 $\mathrm{u}_{\mathrm{rms}} / \mathrm{U}_{-} 0$ (solid line: simulations with $90 \times 150$ and $\mathrm{dt}=0.01 \mathrm{~s}$; symbols: DNS

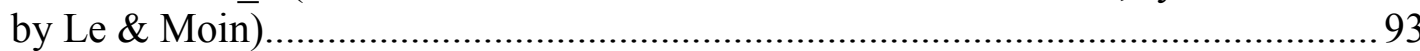

Figure 5-12 $\mathrm{v}_{\mathrm{rms}} / \mathrm{U}_{-} 0$ (solid line: simulations with $90 \times 150$ and $\mathrm{dt}=0.01 \mathrm{~s}$; symbols: DNS by Le \& Moin) 94

Figure 5-13 wrms/U_0 (solid line: simulations with 90x150 and $\mathrm{dt}=0.01 \mathrm{~s}$; symbols: DNS

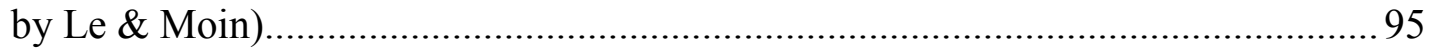

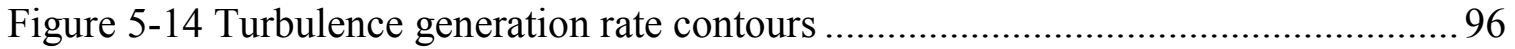

Figure 5-15 Normalized turbulent kinetic energy contours ........................................ 97

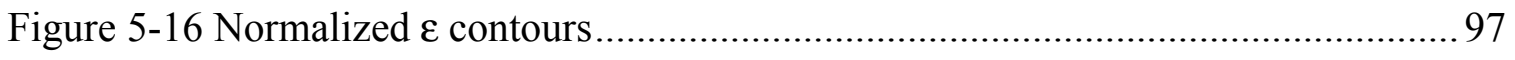




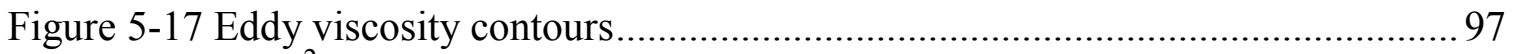

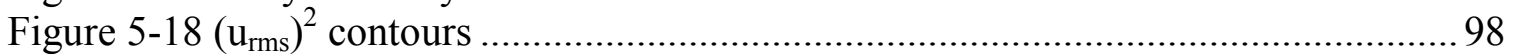

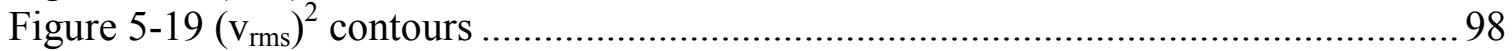

Figure 5-20 Schematic view of the computational domain for the square cylinder flow

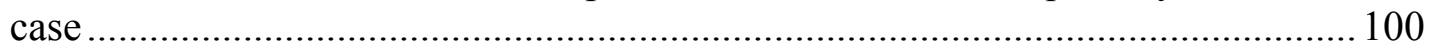

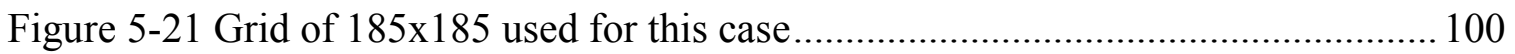

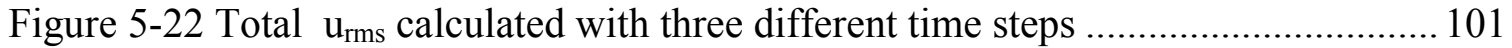

Figure 5-23 Time variation of the drag coefficient with $185 \times 185$, cubic model ............ 102

Figure 5-24 Example of the spanwise vorticity contours at one time instant ................ 105

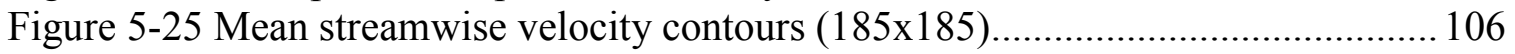

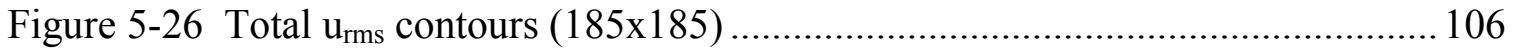

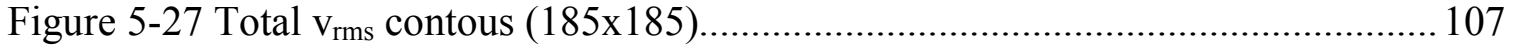

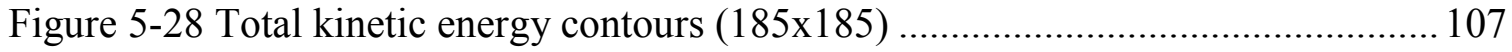

Figure 5-29 Time-mean velocity $<U>$ along center-line of square cylinder ................. 109

Figure 5-30 Total $\mathrm{u}_{\mathrm{rms}}$ along the center-line of the square cylinder ........................... 110

Figure 5-31 Total $\mathrm{v}_{\mathrm{rms}}$ along the center-line of the square cylinder ............................. 110

Figure 5-32 Total turbulent kinetic energy along center-line of the square cylinder...... 112 


\section{List of Tables}

Table 3.1 Different meshes used for the grid convergence study 24

Table 3.2 Turbulent kinetic energy and concentration at the breathing zone for the sharp

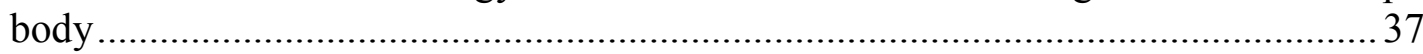

Table 3.3 Reynolds number at different ventilation intensity .......................................52

Table 5.1 Reattachment lengths calculated with different grids and time steps ..............88

Table 5.2 Extrapolated with different methods based on time step variations ................ 89

Table 5.3 Reattachment lengths calculated with different grids and time steps, and extrapolation with different methods based on grid variations

Table 5.4 Global parameter for flow past square cylinder ( $\mathrm{UW}=$ upwinding, $\mathrm{CD}=$ central differencing, $\mathrm{WF}=$ wall function, $\mathrm{NS}=$ no slip, (1)=adjusted for different blockage, (2) $=$ outermost mesh with embedded meshes) (Rodi, 2001)

Table 5.5 Characteristic parameters calculated with the cubic model ........................... 105 


\section{Introduction}

In this study, three dimensional computational fluid dynamics (CFD) simulations are used to investigate the distribution and level of contaminant concentrations in the breathing zone when airborne contaminants are released within an arm's-length in front of the worker who has his back to the airflow. The objective was to gain a better understanding of the fluid motion and mixing phenomena controlled by the combinations of convection, diffusion and buoyancy in the wake of a human body. A concurrent experimental work was carried out in Industrial and Management Systems Engineering Department, West Virginia University (Guffey, 2004). Some results from the experiments were used to validate the simulations.

\subsection{Worker exposure in a ventilation tunnel}

Ventilation systems are used to create a healthy indoor air quality and a comfortable indoor thermal condition with as low as possible energy consumption. Wind tunnels are widely used to study human exposures to airborne contaminants since they allow investigators to control airflow conditions.

In the concurrent experimental study, a wind tunnel is utilized with an inlet honeycomb grid to straighten the flow and to induce turbulence. Gaseous contaminants (ethanol vapor) are released within an arm's length of the worker who has his back to the flow. The Reynolds number based on the equivalent dimension of the torso is in the range of $2000-30,000$ (600 - 10,000 if based on the equivalent dimension of the head). With a heated manikin, the Grashof number is about $8 \times 10^{8}$. The exposure level is measured by the concentration at the breathing zone, which is represented by a single point one centimeter from the mouth center in the current numerical study.

Generally, the ventilation flow in the wind tunnel is of an incompressible, non-isothermal type with heat and mass transfer. The flow over a human body involves a very complicated flow due to the irregular body configuration. There is not much information about this kind of wake flow in the literature. However, the basic characteristics can be revealed by studying the wake flow of a cylinder and a sphere, since the head and the torso could be approximated by a sphere and an elliptic cylinder, respectively. 
Fortunately, these flows have been of great interest for researchers. The following is a brief review about the flow character of these two classical flows.

The experimental work by Tritton (1959) shows that at a Reynolds number of 150, the vortex street becomes turbulent in the wake downstream of a circular cylinder and at a Reynolds number of 400 , the vortices become turbulent after the separation point somewhere in the wake formation region. The experiments by Achenbach (1968), on the other hand, indicate that the transition from laminar flow to turbulent flow in the boundary layer can take place even at low Reynolds number. The laminar boundary layer will become turbulent when the Reynolds number reaches a critical value around $2 \times 10^{5}$. Thereafter, the boundary layer will separate farther rearward than if it were laminar.

The variation of the sphere wake structure with Reynolds number is as follows. When the Reynolds number is lower than about 20, the flow is laminar everywhere and separation does not occur (Taneda 1956). At Reynolds numbers between about 20 and 400, a stationary, symmetric pair of vortex ring is formed at the rear of the sphere (Taneda 1956; Achenbach 1974). When a Reynolds number of about 400 is reached, the vortex ring begins to oscillate, and the wake forms horseshoe-shaped vortex loops at Reynolds numbers between about 400 and 1000 (Achenbach 1974). At Reynolds numbers above about 1000 the vortex loops diffuse very rapidly, which indicates a turbulent flow field in the wake formation region (Taneda, 1978). Similarly, at a critical Reynolds number around $10^{5}$ the laminar boundary layer becomes turbulent before separation.

The above information indicates that most probably a turbulent wake flow is formed in the downstream of a human body after a laminar boundary separation. An unsteady vortex shedding may appear in the wake. However, the vortex may diffuse rapidly because of the flow between the arm and the torso.

\subsection{Factors related to exposure levels}

The worker exposure in a wind tunnel could be affected by a great number of complicating factors, many of which are not yet fully understood. These factors which could affect the worker exposure significantly in a wind tunnel are investigated. These factors include: the contaminant position, the 
orientation of the worker, the ventilation intensity, the level of free stream turbulence, the heat flux from the body, the body shape and the flow unsteadiness. Understanding the effects of these factors on the flow field and the worker exposure will be important for exposure control.

\subsection{Turbulence models for worker exposure}

To date, there are three main classes of approaches for dealing with turbulent flows: Reynolds Averaged Navier-Stokes (RANS), Large Eddy Simulation (LES) with a sub-grid scale (SGS) model, and Direct Numerical Simulation (DNS) with any turbulence model. DNS and LES are still too computationally expensive to be employed on a regular basis, and, from an engineering point of view, they provide far more information than an engineer needs.

Recently, various RANS models are used to deal with the complex turbulent flows in work places. The most widely used RANS models are those linear eddy-viscosity models with wall functions because of their robustness, economy, and reasonable accuracy for a wide range of turbulent flows. However, as it is well known, the linear eddy-viscosity models (EVM) have certain deficiencies. Most importantly, the Boussinesq hypothesis which these models are based on fails when non-trivial flows are considered (e.g., flows over curved surfaces, flows in ducts with secondary motions, flows in rotating fluids, and flows with separation). Various modifications and new modeling concepts have been proposed over the past decades, ranging from ad hoc remedies, and complex non-linear eddy-viscosity approaches to Reynolds stress models/second-moment closures. Guidance and recommendations on the application of these models for exposure prediction would greatly ease the usage of CFD in the industrial hygiene community.

\subsection{Motivation and objectives}

In this study, three dimensional computational fluid dynamics (CFD) simulations are used to calculate the concentration of gaseous contaminants in the breathing zone of a worker when airborne contaminants are released within an arm's-length in front of a manikin who has his back to the airflow. The main objectives were to numerically evaluate the effect of different factors on the manikin exposure and to recommend a turbulence model preferable for this type of simulations. These factors investigated include 
the body shape, the heat flux from the body, the ventilation intensity, the level of free stream turbulence, and the flow unsteadiness. 


\section{Literature review}

To accurately predict the exposure levels in the working environment, one has to understand which factors are significant for work exposure and what kind of models one could use to account for these significant factors. In this literature study, two issues are of major concern. One is the factors which can impact the exposure levels in the working environment, such as the ventilation intensity. The other is modeling-related issues. Simulating work exposure is a complicated problem which comprehends turbulent flow with complex geometry, heat transfer, and mass transfer. That is why it is important to understand the pros and cons of different models for turbulence, heat transfer and mass transport. The choice for predicted worker exposure should be made based on which model can best solve this problem.

\subsection{Factors affecting exposure levels}

The worker exposure in a wind tunnel could be affected by a great number of complicating factors, many of which are not yet fully understood. A list of factors which are important include the following:

- Orientation of the worker

- Body shape

- Ventilation intensity

- Heat flux from the body

- Contaminant source position

- Flow unsteadiness

- Position and configuration of facilities

- Contaminant material and momentum

- Free stream turbulence

- Worker motion 
This is a formidable list, and most of these continue to be the topics of research in this field. Nevertheless, all these factors which prove significant should be considered to accurately predict the exposure levels.

It is not possible to give a thorough discussion and literature review for each of these topics individually. However, a brief introduction and review with respect to six of the general and controllable of these factors will be given next. The topics and factors that are believed to be the more general and the more controllable in both the experiments and simulations include the orientation of the worker, the contaminant source position, the body shape, the ventilation intensity, the heat flux of the body, the level of free stream turbulence, and the flow unsteadiness.

The position and the configuration of the facilities, the contaminant material, size, location and momentum, and the worker motion vary significantly in different applications. In this study, these factors are fixed so that the focus is on the flow field in the wake of the human body and its relation with the exposure levels. A still fixed manikin was used in an otherwise empty wind tunnel. Gaseous contaminant was used to represent the contaminant source since it follows the flow closely.

\section{Orientation of the worker}

Although the situation -- flow from the back of a worker is the main concern of this study -- some attention should be also given to the influence of the orientation of the worker because of its extreme significance. Experimental studies by George et al. (1990), Kim and Flynn (1991), Carlton and Flynn (1997), Welling et al. (2000), and Guffey et al. (2001) have shown that much higher contaminant concentrations occur at the back-to-flow orientation than when air flows from the side or from the front of the subject. Flynn and Ljungqvist (1995) reported that the reason for higher exposure levels at the back to flow orientation is that a reverse flow wake region is created downstream of the worker and this recirculation will bring the contaminant to the breathing zone.

In this numerical study, the flow from the back is considered for all cases since it is considered the worst case when the worker has a hand-held contaminant source. 


\section{Body shape}

The effects of different body shapes on fluid flow and concentration patterns around the body in a wind tunnel have been evaluated to clarify if a sharp body or a block could be a surrogate for the human form in consideration of occupational and environmental health studies. Simple body shapes such as rectangular body, cylinder body and the composition of simple geometries have been widely used, despite lack of positive proof that they are capable of representing the real human body well. Brohus and Nielsen (1996) investigated the effects of body shapes using three different models, all of which were made of rectangular geometries and are insufficient to simulate a real human body accurately. In this study, a rounded body was generated to represent a human-like manikin used in the experiments as closely as possible in order to obtain accurate information about the flow field behind the manikin, especially the separation and the reattachment of the flow around the head which may be significant in evaluating the worker exposure (Li et al. 2003).

\section{Ventilation intensity}

Usually, the wind speed in a ventilation tunnel is 0.1-1.0 m/s (Baldwin and Maynard, 1998), which is comparable with the face velocities of local ventilation hoods. A lot of experiments have been done in this wind speed range.

The experiments by George et al. (1990) reported that the contaminant concentration will decrease as the free stream velocity increases if the worker stands with his back towards the flow and there is no obstruction in the wake zone downstream of the body. However, this trend may not be followed if some object obstructs the flow field downstream of the body.

The experimental work of Saamanen, et al. (2002) showed that the breathing-zone concentration increases in the freestream velocity range of $0.1-0.3 \mathrm{~m} / \mathrm{s}$ when the free stream air velocity increases. However, they used a simplified manikin which was composed of boxes. Their numerical simulations gave results which agree poorly with the experimental results.

The numerical analysis on the effect of inlet velocities on the exposure level may help to optimize the running conditions of the wind tunnel and alleviate the economic limitations to some extent. On the 
other hand, the current study is also used to validate the turbulence models by comparing the numerical results with the experiments.

\section{Free stream turbulence}

The turbulence intensity in the work places varies significantly. Experiments (Hancock and Bradshaw, 1983 and 1989; Kondjoyan, 2002) have shown that the free stream turbulence could significantly affect the transition from laminar to turbulence and thereafter the heat flux from the wall to the fluid because of its interaction with the boundary layer. Péneau et al. (2000) have recovered the same results using large eddy simulation. It could be an interesting topic to investigate the effect of the free stream turbulence intensity on the worker exposure. However, to the best of author's knowledge, there is little information in the literatures on this topic.

Welling, et al. (2000) measured the free stream air velocities, turbulence intensities and temperatures at the beginning of each experiment at 280 symmetrically distributed points, and they found that the free stream velocities and turbulent intensities changed significantly at low wind speed $0.1 \mathrm{~m} / \mathrm{s}$. They also observed that the variation of the nose concentration at that wind speed is about $90 \%$. The reason is obvious due to the variation of the mean free stream velocity and the turbulent intensity. However, it's hard to differentiate the effect of the mean velocity and the turbulent intensity because these two factors were not controlled separately.

A thorough numerical analysis can be used to isolate the effect on the exposure levels from these two factors since it is easy to control these factors in numerical simulations.

\section{Heat flux from the body}

The heat convection from the body may affect the worker exposure in three possible ways: (1) it can transport the contaminants from the waist to the breathing zone by the action of buoyancy; (2) it may intensify the turbulence in the flow field and result in more diffusion; (3) it may cause contaminants that are "trapped" in the recirculation region near the breathing zone to rise out of that region into the free stream. Some experimental and numerical work in the literature, such as Saamanen, et al. (2002), Li, et al. (2003), Hyun and Kleinstreuer (2001) have shown that the buoyancy is important at low free stream 
velocities. The current study will concentrate on how the nature convection affects the flow field and thereafter the concentration field near the mouth at different Reynolds numbers.

\section{Flow unsteadiness}

Large scale unsteady motion is expected to be formed in the downstream of the body as mentioned in Section 1.1, which may result in the large variation of the exposure level at the breathing zone. Although the variation of the instantaneous exposure levels has been observed by other researchers (Welling, et al. 2000), its relation to the flow dynamics in the body wake has not been documented well. The current flow unsteadiness study utilizing LES is aimed to understand the flow dynamics in the formation and the developing of the wake flow in the downstream of human body as well as the relation to the contaminant transport. Hopefully, it will also provide some guidance on the sampling period for the experimental study.

\subsection{Modeling related issues}

There are three main classes of numerical techniques for dealing with turbulent flow: Reynolds Averaged Navier-Stokes (RANS), Large Eddy Simulation (LES) and Direct Numerical Simulation (DNS). DNS resolves the Navier-Stokes equations without averaging and approximation. It is viewed as an indispensable research tool to help the understanding the physics of the flow and the developing of turbulence models. However, it is simply too expensive to be employed on a regular basis, and, from an engineering point of view, it provides far more information than an engineer needs.

LES has gained overwhelming consideration in both industry and academy. The physical basis for LES is that the large-scale motions in the turbulent regime are more energetic than the small scale ones and are responsible for most of the transport. The small-scale turbulence is more universal and nearly isotropic, which makes it more suitable to be modeled. Mathematically, LES is applied by filtering the Navier-Stokes equations. The filtered quantities are modeled by a so-called subgrid scale model which relates the effects of small eddies to the resolved large eddies. Still, LES is constrained by the near-wall resolution requirements. For instance, to resolve the near-wall streaky structures, LES requires a grid density close to that for DNS with spacing of the order $\Delta \mathrm{y}^{+}=\mathrm{O}(1), \Delta \mathrm{x}^{+}=\mathrm{O}(50)$ and $\Delta \mathrm{z}^{+}=\mathrm{O}(20)$, where $\mathrm{x}^{+}, \mathrm{y}^{+}$, and $\mathrm{z}^{+}$ denote the streamwise, wall-normal and spanwise direction in wall unit respectively. As a result, one has to 
wait a long while (until 2045 according to Spalart, 2000) to use LES in large scale industrial and environmental applications (e.g., flow over a car).

Turbulence modeling with RANS equations has started nearly a century ago, when Boussinesq introduced the concept of an eddy viscosity (i.e. the Boussinesq eddy viscosity approximation, which relates the Reynolds stresses to the shear rates). Nowadays the field of classical RANS turbulence modeling is still active despite its disputable intuitive and empirical rationale. They are simple to use, computationally affordable and economical, thus appealing to industry for various applications, such as design, optimization, and prediction of off-design performances. They do not simulate the details of the turbulent motion, but only the effect of turbulence on the mean flow behavior. The following is a literature survey on advanced RANS turbulence models.

\subsubsection{Advanced RANS turbulence models}

The most popular and most widely used linear eddy-viscosity models with wall functions are used to predict the worker exposure because of its robustness, economy, and reasonable accuracy for a wide range of turbulent flows. However, as it is well known, the linear eddy-viscosity models (LEVM) have certain deficiencies, and the Boussinesq hypothesis fails when non-trivial flows are considered (e.g. flows over curved surfaces, flows in ducts with secondary motions, flows in rotating fluids, and flows with separation). Various modifications and new modeling concepts have been proposed over the past decades ranging from ad hoc remedies, complex non-linear eddy-viscosity approaches to Reynolds stress models/second-moment closures.

Here a summary is presented of these turbulence models which show superiority over the linear eddy-viscosity models. One of these models which have potential to improve the predictions on worker exposure will be chosen and implemented in the DREAM code (Celik and Badeau, 2003). Its performance will be evaluated with various flows such as turbulent channel flow, flow over a backward facing step, and flow over square cylinder. Since the exposure prediction involves mass and heat transport in a complex geometry, there are certain concerns which will be addressed in this summary. First, the near wall techniques will be one of the major concerns since it is important for separation, reattachment and the heat transfer on the wall. Secondly, the models for the buoyancy-driven flow will be presented because they 
may be significant for accurate predictions of flow details and wall heat transfer. Last, the numerical stability of these turbulence models is discussed since it is always unavoidable especially in cases involving complex geometry.

\subsubsection{Non-linear $k-\varepsilon$ turbulence model}

The alternative to the simple linear eddy-viscosity model is the nonlinear relationship for approximating the Reynolds stresses which adopts an algebraic expression between stress and strain. Such relationships may be arrived at by simplifying stress-transport models (so-called algebraic stress models, ASMs). In view of the current limitations of such simplification, it may be better to regard them simply as conjectured generalizations of the eddy-viscosity approach, containing quadratic and, occasionally, higherorder products of the strain and vorticity tensors.

Quadratic models have been proposed by Speziale (1987), Nisizima \& Yoshizawa (1987), Rubinstein \& Barton (1990), Myong \& Kasagi (1990) and Shih et al. (1993). All of these proposed models arrived at the empirical coefficients by considering the prediction of shear stress in a simple shear and one other complex flow (or some other feature of a simple shear -such as the normal stress level - that can not be mimicked with a linear scheme). However, Craft et al. (1996) argued that there seems be little agreement between them on coefficient values, which implies that, at quadratic level, only slightly greater generality is achievable than with the usual linear eddy-viscosity model. In particular, the effects of streamline curvature and swirl on the turbulent stresses cannot be adequately accounted for at this level.

Craft et al. (1996) developed the non-linear eddy-viscosity model including low-Reynolds-number

effect. This model was tested to predict a range of applications, including flow in curved channels, impinging jet flow etc. In each case it resulted in some predictive improvements in comparison to that produced by a linear low-Reynolds-number k- $\varepsilon$ model. Moreover, they demonstrated that, in order to exhibit the correct sensitivity to streamline curvature, such a non-linear model must retain cubic terms in the stress-strain relationship.

One problem found with this cubic model is that it couldn't predict enough stress anisotropy in the near-wall regions. Abe et al. (2003) included additional terms in the stress-strain relation, dependent on the 
wall-distance, to force greater levels of stress anisotropy near a wall. However, the scheme does rely on the wall distance which is not an ideal parameter to use in flows with complex geometries. Suga (1995) proposed another transport equation to solve the anisotropy which doesn't need wall distance but requires computational effort.

Another problem with Craft's cubic model (1996) was found by Iacovides and Raisee (1997). He showed that severe problems of numerical stability were encountered with applications of this model in the computation of heat and fluid flow through ribbed passages. He traced these stability problems to the form of the dependence of $\mathrm{C} \mu$ on the strain rate, which, in flows over sharp corners, led to very abrupt changes in turbulent viscosity. Craft et al. (1999) therefore introduced two modifications to their original model: (1) the introduction of an alternative formulation for the turbulent viscosity parameter, $\mathrm{C}_{\mu}$, with the strain rate, and (2) the replacement of the Yap algebraic length-scale correction term with a modified form of Iacovides and Raisee (1999) differential length-scale correction term. This proposed model not only improved the heat transfer predictions in both pipe-expansion and impinging jet, but also removed the need for an explicit wall distance to be prescribed in the model. Raisee et al. (2004) tested this modified model and found that it produced reliable thermal predictions in ribbed cooling passages.

It is worth noting here that computing times required for non-linear model are typically only 10$20 \%$ more than for a linear eddy-viscosity model.

\subsubsection{Reynolds stress model (RSM) /Second momentum closure (SMC)}

Second momentum turbulence models focus directly on the transport equations for the Reynolds stresses rather than supposing the stress and strain fields to be directly linked via an eddy viscosity. This elaboration enables the effects of complex strains and force fields on the turbulence structure to be better captured. The RSM model is better in theory than other models because physically it contains terms accounting for effects of non-isotropy, curvature, and extra rate of strain etc.

The equations are unclosed, however, so the task of the RSM/SMC turbulence models is to devise approximations for the unknown turbulence correlations in terms of known or determinable quantities. For instance, the pressure strain $R_{\mathrm{ij}}$ (see Eq.3.15) is the most important part that needs to be modeled. Many 
different pressure strain models have been proposed, including the HL model of Hanjalic and Launder (1972); the quasi-isotropic model (LRR-QI) of Launder et al. (1975); the SL model of Shih and Lumley (1985); the JM model of Johns and Musonge(1988); the SSG model of Speziale, Sarka, and Gatski (1991); and the FLT model of Fu, Launder and Tselepidakis(1987).

Launder (1989) compared the performance of SMC and linear EVM. He found that the SMC generally assures a prediction which is better, or at least similar, results as the linear EVM. He also pointed out that the determination of $\varepsilon$ (the dissipation rate) has been the weakest point in the SMC model because there is not a directly useful exact equation to serve as a framework for modeling. Lien and Leschziner (1994) examined the Reynolds stress models with a separated flow behind a backward-facing step. They found that second-moment-closure variants resolve well, in contrast to linear k- $\varepsilon$ models (including the RNG version), the secondary corner eddy.

The difficulty which has discouraged many researchers from venturing to use the Reynolds stress models is the numerical instability, which was reported by Amano and Goel (1984), and Huang and Leschziner (1985). There are many sources which could contribute to this problem, including (1) the turbulent Reynolds stresses as source terms in the momentum equations; (2) the coupling of all the Reynolds stress equations. The following is a summary of special considerations for implementing RSM.

\section{Numerical consideration for Reynolds stress models}

Launder (1989) noted that if one adopts the staggered velocity/pressure node cluster, numerical stability is increased and the amount of interpolation required is decreased if the stresses are also staggered, (i.e. the normal stresses are located at the scalar node while the off-diagonal components are positioned so that they lie on the boundaries of the control volumes of the velocity components).

In a three-dimensional flow, each stress component appears in the budget equations for many of the other components. This strong intercoupling suggests that one should adopt for a simultaneous solution of the six components at a point. However, such a direct approach is spectacularly unstable, partly, because the stresses are scattered. The strategy is first to solve for the three coincident normal stresses, interpolating as necessary the 'old' values of the shear stresses. When updated values of the normal stresses have been obtained at all nodes, the off-diagonal components are obtained by a pointwise substitution. 
The turbulent shear stress is often several orders larger than the molecular shear stress in turbulent flows. Treating it as a large source term creates stiffness and hence causes numerical instability in the numerical iterations.

In simple shear flow calculations the turbulent shear stress $\overline{-\mathrm{uv}}$ in the momentum equation is usually divided and multiplied by the velocity gradient

$$
\partial(-\overline{u v}) / \partial y=\partial\{[(-\overline{u v}) /(\partial U / \partial y)] \times(\partial U / \partial y)\} / \partial y
$$

and $(\overline{-u v}) /(\partial \mathrm{U} / \partial \mathrm{y})$ is treated as the eddy viscosity. In doing this, the turbulent shear stress is effectively cast into the diffusion term.

However, Hwang and Peng (1995) pointed out that in elliptic-type flows the velocity gradients will not always have the same sign as the turbulent shear stress as they do in simple shear flows. When the velocity gradient and the turbulent shear stress are of opposite sign, negative nodal coefficients will be introduced, which can lead to numerical instability.

A technique that could be used to overcome the numerical instability of the momentum equations is a modification of the numerical procedure for the eddy viscosity model. The Reynolds stresses can be expressed as

$$
-\overline{u_{i} u_{j}}=-\overline{u_{i} u_{j}}+C_{\mu} \frac{k^{2}}{\varepsilon}\left(\frac{\partial U_{i}}{\partial x_{j}}+\frac{\partial U_{j}}{\partial x_{i}}\right)-C_{\mu} \frac{k^{2}}{\varepsilon}\left(\frac{\partial U_{i}}{\partial x_{j}}+\frac{\partial U_{j}}{\partial x_{i}}\right)
$$

where $C_{\mu}=0.09$. The second term is cast into turbulent diffusion forms in the numerical solution, and the rest into source term, which is trivial and have no serious effect on the numerical stability of the momentum equation.

Computations with a SMC model will probably require 50-200\% more computer time than a linear two-equation eddy viscosity model. 


\subsubsection{Elliptical relaxation turbulence model}

Noting that the appropriate velocity scale for turbulent transport toward the wall is $\overline{v^{2}}$ other than $\mathrm{k}$, Durbin (1991) proposed an elliptical relaxation model (so called $\overline{v^{2}}-f$ model) for the strongly heterogeneous region near the wall. The $\overline{\mathrm{v}^{2}}-\mathrm{f}$ model consists of three transport equations for the turbulent kinetic energy $k$, the dissipation of the turbulent kinetic energy $\varepsilon$, and a transport equation for the energy of the fluctuations normal to the streamlines $\overline{\mathrm{v}^{2}}$. In addition, the model includes a Helmholtz type equation for a quantity $\mathrm{f}$ which models the pressure-strain term. This equation is elliptic in nature, and as a consequence information from all spatial directions is used to compute the variable, $\mathrm{f}$, at a given point.

IN recent years the $\overline{v^{2}}-f$ turbulence model has been tested against many flows and has become increasingly popular due to its ability to account for near-wall damping without use of ad hoc damping functions. Durbin (1993) tested this model on turbulent separated flows over a backward facing step, in a plane diffuser, and around a triangular cylinder. He found that the $\overline{v^{2}}-f$ model is viable for highly nonequilibrium turbulent flow. Lien et al. (1998) showed that the performance of the $\overline{\mathrm{v}^{2}}-\mathrm{f}$ model for a flow over compressor-cascade blades, involving both the leading-edge and trailing-edge separation, is very encouraging. The imposition of a realizability constraint on turbulence scales significantly reduces the level of turbulence energy at the stagnation region. Although the size of the resulting laminar separation bubble was slightly too large, the velocity profiles close to the trailing edge were in good agreement with the experimental data. Iaccarino and Durbin (2000) applied $\overline{\mathrm{v}^{2}}-\mathrm{f}$ to the solution of the flow around a surface mounted cube. They confirmed that the steady flow simulation was not adequate to correctly reproduce the essential physics associated with massively separated flows. Their prediction with the unsteady RANS with the $\overline{v^{2}}-f$ turbulence showed significant agreement with the experimental results.

Although the $\overline{v^{2}}-f$ model has shown superiority over linear EVMs, special requirements should be considered to implement it. These equations should be solved two at a time as coupled k- $\varepsilon$ and $\overline{\mathrm{v}^{2}}-\mathrm{f}$ systems. That makes the equations sufficiently implicit to permit large pseudo-time steps in steady 
state calculations without numerical instability. Durbin (1995) pointed out that the coupling between pairs of equations is dictated primarily by the no-slip boundary condition.

Sveningsson (2003) modified the $\overline{v^{2}}$ and $f$ equations so that $\mathrm{f}$ is zero at wall. He showed that both

of the original and the modified $\overline{v^{2}}-f$ models tested give very good heat transfer distribution, indicating the promising near-wall modeling using the elliptic relaxation methodology. However, a coupled solver is still required for this modified model.

Hanjalic et al (2004) proposed a $\xi-f$ model based on Durbin's elliptical relaxation concept, which solves transport equation for the velocity scales ratio instead of the equation for $\overline{\mathrm{v}^{2}}$. It has two advantages over the original $\overline{\mathrm{v}^{2}}-\mathrm{f}$ model: (1) instead of $\varepsilon$ appearing in the $\overline{\mathrm{v}^{2}}$ equation, the $\xi$ equation contains the turbulent kinetic energy production, $\mathrm{P}$, which is much easier to reproduce accurately if the local turbulent stress and the mean velocity gradient are captured properly; (2) the near wall value of $f$ is proportional to $\mathrm{y}^{2}$ instead of $\mathrm{y}^{4}$ as in the original model, which improve stability of the computational scheme. Similar quality of predictions with $\xi-\mathrm{f}$ model was obtained as with the original $\overline{\mathrm{v}^{2}}-\mathrm{f}$ model when they were tested with a plane channel flow, a separating flow behind a backward facing step, and a round impinging jet.

\subsubsection{Near-wall approaches}

The overall success of all turbulence models in wall bounded internal as well as external flows is determined in large measure by the treatment of solid walls. Three different ways are commonly employed to compute the near-wall behavior of complex turbulent flow. They are wall functions, low-Reynolds number model (LRN), and two layer models.

\subsubsection{Wall functions}

In application of turbulence models, the most popular near-wall treatment is the wall function method because it requires much less grids $\left(30<\mathrm{y}_{1}^{+}<60\right)$ in the near wall region. Wall functions are derived on the assumption that a Couette flow prevails, i.e. that the variation of the dependent parameters with 
respect to the streamwise direction can be neglected. For impermeable walls the flow equations can be solved analytically to yield the well known, law-of-the-walls:

$$
\begin{aligned}
& U^{+}=\frac{1}{k} \ln \left(y^{+}\right)+B \\
& T^{+}=\frac{\operatorname{Pr}_{t}}{\kappa} \ln \left(y^{+}\right)+B_{T}
\end{aligned}
$$

Employing these equations permits one to simplify the near-wall treatment to a one-dimensional analysis for which the solution of the dependent variables is only a function of the normalized wall-distance. Naturally such a simplification reduces the computational storage and time through a reduction of the number of nodes. In addition, the convergence rate is increased as a result of the simplified equations.

Unfortunately, the universality of these wall functions is limited since they are derived from simplified governing equations. For instance, calculations of backward facing step flows with wall functions lead to more than $20 \%$ error, in the reattachment length (Rodi et al 1993). Although the basic assumptions made in the wall function approach, (i.e., flow parallel to the wall and equilibrium turbulence relations), are correct for simple wall shear flows, a certain error will be created when the method is applied to complex wall shear flows with separated regions.

\subsubsection{Two-layer model}

In two-layer models the near-wall viscosity-affected regions are resolved, such that the dissipation rate of the turbulent kinetic energy is determined by a prescribed length scale distribution instead of by the transport differential equation.

The turbulent eddy viscosity in the near wall region is given by

$$
v_{t}=C_{\mu} k^{1 / 2} l_{\mu}
$$

and $\varepsilon$ is determined from 


$$
\varepsilon=k^{3 / 2} / l_{\varepsilon}
$$

The length scales $1_{\mu}$ and $1_{\varepsilon}$ are adopted from the model

$$
\begin{aligned}
& l_{\mu}=C_{l} y\left[1-\exp \left(-R_{y} / A_{\mu}\right)\right] \\
& l_{\varepsilon}=C_{l} y\left[1-\exp \left(-R_{y} / A_{\varepsilon}\right)\right]
\end{aligned}
$$

where both length scales express damping effects in the near-wall region in terms of the turbulence Reynolds number $\mathrm{R}_{\mathrm{y}}=\mathrm{k}^{1 / 2} \mathrm{y} / \mathrm{v}$. Here $\mathrm{y}$ is the normal distance from the wall. The turbulence model moduli are given as $\mathrm{C}_{1}=\kappa \mathrm{C}_{\mu}{ }^{3 / 4}, \mathrm{~A}_{\varepsilon}=2 \mathrm{C}_{1}$ and $\mathrm{A}_{\mathrm{k}}=70$, where $\kappa$ is the Karman constant and $\mathrm{C}_{\mu}=0.09$.

In conducting the computation, the two models have to be matched at some location in a region where viscous effects have become negligible. In general, preselected grid lines are set for matching the two models within the criterion $\mathrm{R}_{\mathrm{y}} \geq 250$ recommended by Chen and Patel (1988).

\subsubsection{Low Reynolds number model}

Over the past few decades, many suggestions have been made for the extension of turbulence models to enable their use at low turbulence Reynolds numbers and to describe the flow close to a solid wall. The simplest example is the van Driest damping function (van Driest, 1956) for the mixing length. More advanced models incorporate either a wall damping effect or a direct effect of molecular viscosity, or both, on the empirical constants and functions in the turbulence-transport equations devised originally for high Reynolds number, fully turbulent flows remote from the walls.

In the literature, a lot of work has been done on the low-Reynolds number two equation models. Patel et al. (1985) reviewed these works and suggested that the better performance of the low Reynolds number model could be achieved by: 1) selecting a damping function, $f_{\mu}$, for the shear stress that is in agreement with experimental evidence and whose influence is restricted to the sublayer and the buffer layer;

2) choosing the low Reynolds number functions $\mathrm{fl}$ and $\mathrm{f} 2$ in the $\varepsilon$ equation with a mathematically 
consistent near-wall behavior; 3) fine-tuning the functions to ensure the reproduction of the well-known basic feature of wall-bounded shear flows over a range of pressure gradients. This suggestion has been followed by many later researchers (Bredberg and Davidson, 2004; Raisee, et al. 2004). They implemented the non-linear turbulence model together with the low Reynolds number model at the wall and the results has been shown to be satisfactory.

It should be noted here that the first interior nodes should be very close to the wall $(y+<5)$ for the low Reynolds number model. The numerical experiments of Bredberg and Davidson (2004) showed that the predicted Nusselt number along the lower wall of a rib-roughened channel with $y_{1}^{+}=1$ and $y_{1}^{+}=4$ meshes could be within $5 \%$ of the grid independent result. Even though the prediction with $\mathrm{y}_{1}^{+}=10$ mesh deviates by more than $25 \%$ from the experimental data, they are still encouraging when compared to other turbulence models, especially when using wall function based models.

\subsubsection{Modeling buoyancy-driven flow}

It is popular to use the simple eddy diffusivity models for turbulent flux of heat $\overline{\theta u_{j}}$ (or species):

$$
\overline{\theta u_{j}}=-\frac{v_{t}}{\sigma_{T}^{t}} \frac{\partial T}{\partial x_{i}}
$$

where $\sigma_{\mathrm{T}}^{\mathrm{t}}$ is the turbulent Prandtl number (or Schmidt number for species) which is a constant or determined from an empirical formulas. It is generally recognized that the linear eddy-viscosity models and their analogues for scalar fields cannot reproduce any flows with significant non-equilibrium effects, flows subjected to body forces, or any extra-strain rates other than simple shear. The above shortcomings can be eliminated by solving the modeled transport equations for $\overline{\theta u_{j}}$, closed by the equations that provide thermal scales (e.g., for $\overline{\theta^{2}}$ and $\overline{\varepsilon_{\theta \theta}}$ ). Differential transport equations have been proposed in the literature and applied with success to the computations of some simple buoyancy-driven flows. However, such models contain many terms that require separate modeling, and deriving a general closure for complex flows is a formidable task. Algebraic models based on a truncation of the differential second-moment 
closure have been proposed as the minimum closure level for complex flows. For instance, Hanjalic proposed a three-equation $\left(\mathrm{k}-\overline{\theta^{2}}-\varepsilon\right)$ model which produced satisfactory solutions in a range of enclosed buoyancy-driven turbulent flows with different geometries and boundary condition. Abe et al. (1995) instead proposed a four-equation model (k- $\left.\mathrm{k}-\overline{\theta^{2}}-\varepsilon-\varepsilon_{\theta \theta}\right)$ which is capable of predicting the heat transfer in separating and reattaching flows downstream of a backward-facing step.

It should be emphasized here that strong variations of all flow properties, in usually very thin boundary layers along the walls where the buoyancy exhibits the strongest effects on turbulence, requires a fine numerical resolution of the near-wall region. Thus, it is indispensable to use a low-Reynolds-number turbulence model which can fully resolve the near-wall region.

\subsubsection{Mass transport models}

When predicting the concentration of contaminants, both Eulerian and Lagrangian methods have been used in the literature. For the Eulerian method, the concentration can be calculated by solving a scalar transport equation, which approximates the turbulent diffusivity in relation with the eddy viscosity with an empirical constant, i.e., the turbulent Schmidt number. Since the contaminant concentration are very sparse for the current study $\left(10^{-3} \mathrm{~kg} / \mathrm{kg}\right.$ air), the effect of the secondary phase (gaseous contaminants) on the main phase (air) is ignored. The Lagrangian method, however, tracks individual parcels in a Lagrangian frame. Each of the parcels represents a certain amount of gaseous contaminant and closely follows the air flow.

Flynn and Sills $(2000,2001)$ used the Lagrangian particle tracking method to predict human exposure to aerosols generated during compressed air spray painting in cross flow ventilated booths. They found that the predicted dimensionless breathing-zone concentrations were in agreement with the measured values.

Longest et al. (2000) applied RANS simulation in conjunction with the Eulerian scalar transport equation to investigate the distribution of $\mathrm{CO}$ in a Rochester-style human exposure chamber. In the current study, the predictions from the Lagrangian and Eulerian methods are compared in order to provide some guidance as to which one is better for predicting human exposure to aerosols. 


\subsubsection{Summary}

In this study, the cubic low-Reynolds-number k- $\varepsilon$ turbulence model (Craft et al. 1996, 1999) was selected because 1) it requires no more PDE's than the standard k- $\varepsilon$ model, which implies that it would not cost much more than the standard k- $\varepsilon$ model; 2 ) it does not have the numerical instability problem which

other advanced models such as RSM and $\overline{v^{2}}$-f model do have and require special strategy. The advantage of the cubic model over linear eddy-viscosity model will be discussed in detail in chapter 4 .

The simple eddy diffusivity models for turbulent flux of heat (Eq. 2.9) is used to predict the thermal field. The models are implemented in the DREAM code which is a 2D/3D flow solver with a staggered grid and a segregated solver. The cubic k- $\varepsilon$ model will be tested on the turbulent channel flow, the backward facing step flow, and the flow past a square cylinder. The results with this non-linear model will be compared to the experimental data and to DNS data, as well as others' predictions with different RANS models and LES. 


\section{The influence of critical factors on worker exposure}

This chapter first presents the computational details such as equations, grids, and the numerical method used in the current work. The results of the grid convergence study are shown next since it is important to know the numerical errors prior to the massive. Then the results of the influence of critical factors are presented and discussed. These results include the isolated influence of the body shape, the ventilation intensity, the level of free stream turbulence, the heat flux of the body, and the flow unsteadiness. Finally, the results are summarized at the end of this chapter.

\subsection{Computational details}

In this study, a three-dimensional wind-tunnel simulation was performed with the Fluent CFD software package (Fluent, Inc., Lebanon, NH). The geometrical configuration of the flow domain is shown in Figure 3.1, consisting of the wind tunnel (4.6m width $\times 2.6 \mathrm{~m}$ height $\times 11 \mathrm{~m}$ length) and a nonbreathing manikin body $(1.8 \mathrm{~m}$ height). The manikin body faces downstream of the flow. Constant inlet velocity inlet and constant pressure at the outlet boundary were used. The inlet velocity was taken as 0.3 $\mathrm{m} / \mathrm{sec}$ with a turbulence intensity of $0.3 \%$ to study the effect of the body shapes and the transport methods, values representative of typical working environments (Baldwin and Maynard, 1998). To investigate the thermal impact of the body, an inlet velocity of $0.1 \mathrm{~m} / \mathrm{sec}$ was also used. The characteristic turbulent length scale was chosen to be $0.01 \mathrm{~m}$, which represents the turbulence generating grid size at the inlet of the tunnel. The dimensions of the wind-tunnel and the sharp body configuration are illustrated in Figure 3.2. The source box is $0.3 \mathrm{~m}$ (length)*0.3m(width)*0.05m(height) in size and the box center is $1.2 \mathrm{~m}$ from the ground and $0.5 \mathrm{~m}$ from the body. In addition, block and rounded body models are created as shown in Figure 3.3 to investigate the effect of the shape of the human-body on concentration levels near the breathing zone. It should be noted here that the breathing zone mentioned in the paper is represented by one point, which is $1.65 \mathrm{~m}$ from the ground and $0.01 \mathrm{~m}$ from the body (i.e. a point directly in front of the mouth at a horizontal distance of $0.01 \mathrm{~m}$ ). 


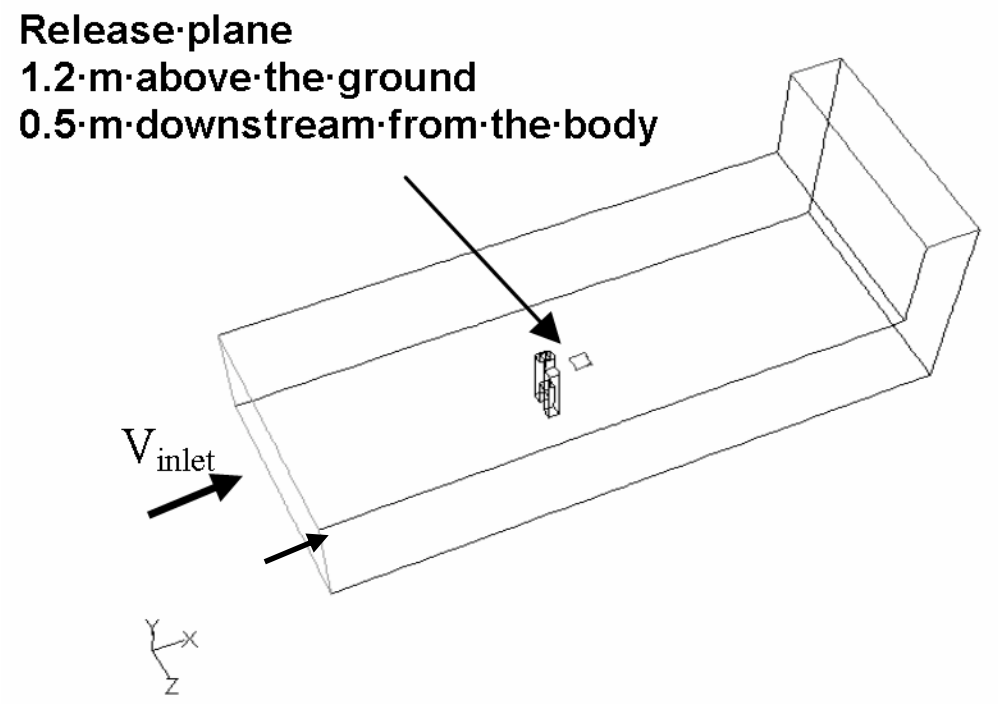

Figure 3-1 Schematic view of the wind tunnel model and location of contaminant release plane

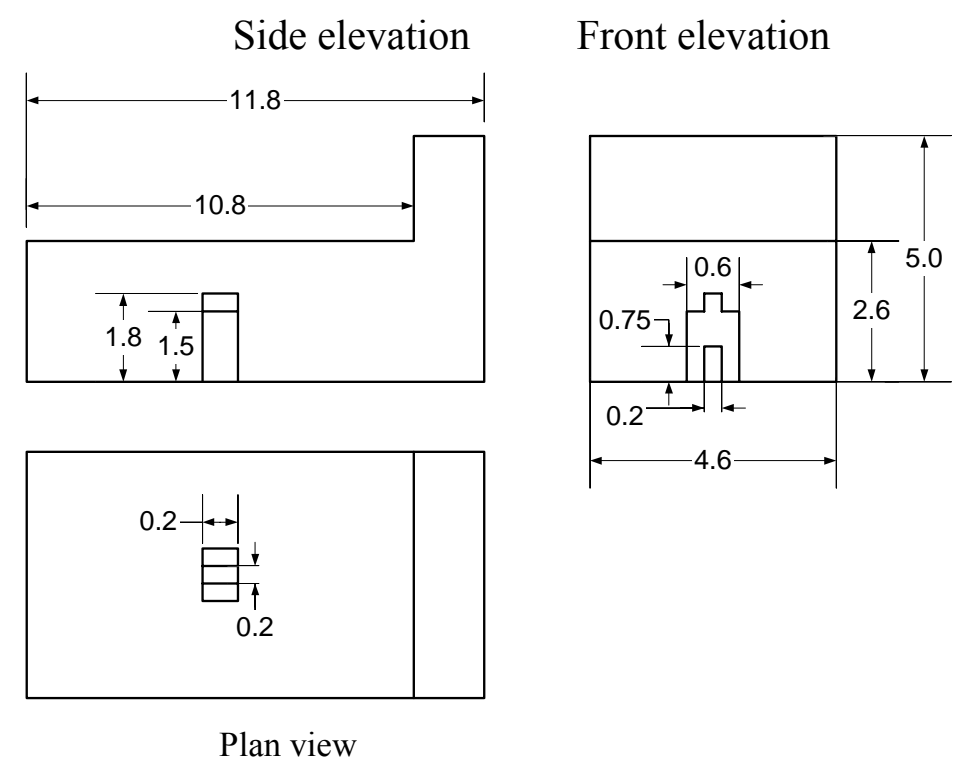

Figure 3-2 The geometry of the wind tunnel and the human body (all dimensions in $\mathrm{m}$ )

Different grid distributions, also known as meshes, were constructed as listed in Table 3.1 in order to quantify the discretization error using Richardson Extrapolation with Grid Convergence Index (GCI) (Roache, 1994) and Extrapolated Relative Error (ERE) (Celik and Li, 2005). Richardson Extrapolation uses calculations on multiple sets of grids to calculate the extrapolated value of a dependent variable to zero grid size, and GCI and ERE use the extrapolated value to determine the numerical uncertainty caused by limited grid size, also known in the literature as the discretization error. For each body, the whole domain was 
divided to several sub-domains each with different grid sizes so that the mesh in the region around the body could be much finer than that in other regions (See Figure 3.4). It should be emphasized that the grids are refined proportionally for each sub-domain to maintain similar grids as suggested by Celik and Karatekin (1997). The only exception was mesh \#3 (the sharp body), for which only the region around the body is refined based on mesh \#2 in the hope to illustrate if better convergence with less cells using non-uniform grids could be achieved.

Table 3.1 Different meshes used for the grid convergence study

\begin{tabular}{|c|l|l|l|}
\hline Mesh \# & \multicolumn{1}{|c|}{ Block body } & \multicolumn{1}{c|}{ Sharp body } & \multicolumn{1}{c|}{ Rounded body } \\
\hline 1 & 125,103 node & 30,621 nodes & 92,166 nodes \\
& 116,712 hexahedral cells & 27,396 hexahedral cells & 339,350 mixed cells * \\
\hline 2 & 229,874 nodes & 124,326 nodes & 136,302 nodes \\
& 217,212 hexahedral cells & 115,824 hexahedral cells & 470,234 mixed cells \\
\hline 3 & & 188,583 nodes & 208,600 nodes \\
& & 169,430 hexahedral cells & 659,110 mixed cells \\
\hline 4 & & 347,595 nodes & \\
& & 330,598 hexahedral cells & \\
\hline 5 & & $1,077,176$ nodes & \\
& & $1,041,318$ hexahedral cells & \\
\hline
\end{tabular}

* Mixed cells mean that some cells are hexahedral cells and the other are tetrahedral cells.

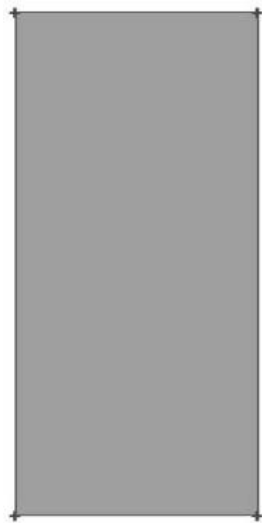

(a) block

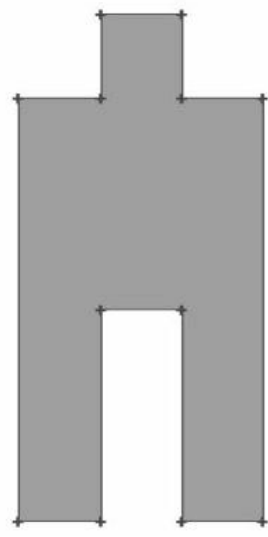

(b) sharp body

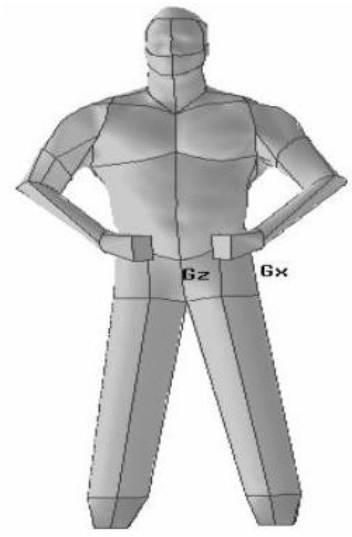

(c) rounded body

Figure 3-3 Different body shapes used in the present study (not to scale)

The heat transfer boundary condition for the human body surface was taken as a convective heat flux of $25 \mathrm{~W} / \mathrm{m}^{2}$ (Bjørn and Nielsen, 1998), which was based on a 2000-calorie diet and corresponds to a 
moderate activity level of a standing person. The fluid medium was air and the contaminant was acetone vapor.

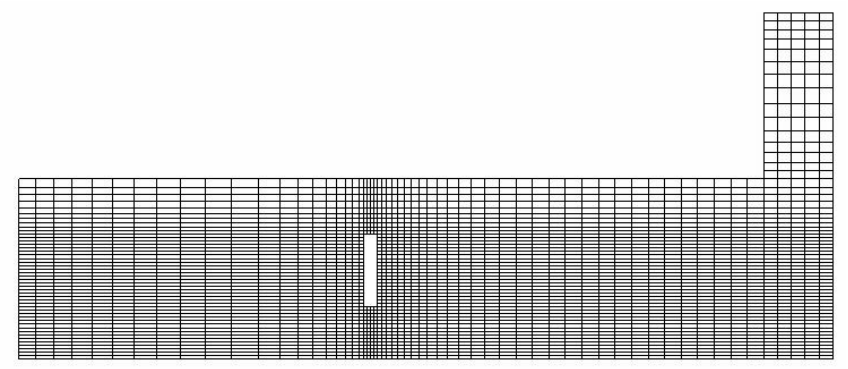

Figure 3-4 An example of a computational mesh used in the simulations (Mesh \#2 for the sharp body, 115,824 hexahedral cells; the empty area represents the center of the sharp body)

The turbulence model of choice was the two-equation standard k- $\varepsilon$ model (Launder and Spalding, 1974), which is one of the most commonly used turbulence models in practical engineering flow calculations. The k- $\varepsilon$ model is based on the eddy-viscosity approximation which expresses the Reynolds stresses in terms of the mean velocity. The shortcomings of the eddy-viscosity approximation has been well documented in the literature (see e.g. Mathieu and Scott, 2000). Numerous more sophisticated models such as Reynolds stress model have been developed to overcome the defects of eddy-viscosity approximation. The choice of a turbulence model for a specific application should be made carefully, depending on the considerations such as the physics encompassed in the flow, the established practice for a specific type of problem, the level of accuracy required, the available computational resources, and the amount of time available for the simulation. In the present study with its complex geometry, the standard k- $\varepsilon$ model was used because of its robustness, economy, and reasonable accuracy for a wide range of turbulent flows.

\subsection{Governing equations}

\subsubsection{Standard turbulence model}

The mathematical formulation of the governing equations and the turbulence models used for steady flow simulations are the time-averaged Navier-Stokes equations with the standard two-equation k- $\varepsilon$ turbulence model. 


$$
\begin{gathered}
\frac{\partial U_{i}}{\partial x_{i}}=0 \\
\frac{\partial U_{i}}{\partial t}+U_{j} \frac{\partial U_{i}}{\partial x_{j}}=-\frac{1}{\rho} \frac{\partial P}{\partial x_{i}}+\frac{\partial}{\partial x_{j}}\left[\left(v+v_{t}\right)\left(\frac{\partial U_{i}}{\partial x_{j}}+\frac{\partial U_{j}}{\partial x_{x}}\right)\right]-\beta\left(T-T_{r e f}\right) g_{i}
\end{gathered}
$$

The Bousssinesq model (Spiegel and Veronis, 1960) is employed for the calculation of the buoyancy force. This model treats density as a constant value in all solved equations, except for the buoyancy term in the momentum equation.

The eddy viscosity, $v_{t}$, is given by

$$
v_{t}=c_{\mu} \frac{k^{2}}{\varepsilon}
$$

The turbulent kinetic energy, $\mathrm{k}$, and its rate of dissipation, $\varepsilon$, are obtained from the following transport equations:

$$
\begin{aligned}
& \frac{\partial k}{\partial t}+u_{i} \frac{\partial k}{\partial x_{i}}=\frac{\partial}{\partial x_{i}}\left[\left(v+\frac{v_{t}}{\sigma_{k}}\right) \frac{\partial k}{\partial x_{i}}\right]+G_{k}+G_{b}-\varepsilon \\
& \frac{\partial \varepsilon}{\partial t}+u_{i} \frac{\partial \varepsilon}{\partial x_{i}}=\frac{\partial}{\partial x_{i}}\left[\left(v+\frac{v_{t}}{\sigma_{\varepsilon}}\right) \frac{\partial \varepsilon}{\partial x_{i}}\right]+c_{1} \frac{\varepsilon}{k} G_{k}-c_{2} \frac{\varepsilon^{2}}{k}
\end{aligned}
$$

and $G_{k}$ is the production rate of turbulent kinetic energy and $G_{b}$ is the generation rate of $k$ due to buoyancy,

$$
\begin{aligned}
G_{k} & =v_{t}\left(\frac{\partial u_{i}}{\partial x_{j}}+\frac{\partial u_{j}}{\partial x_{i}}\right) \frac{\partial u_{j}}{\partial x_{i}} \\
G_{b} & =\beta g_{i} \frac{v_{t}}{\operatorname{Pr}_{T}} \frac{\partial T}{\partial x_{i}}
\end{aligned}
$$

where $\operatorname{Pr}_{\mathrm{T}}$ is the turbulent Prandtl number for energy and is equal to 0.85 . It should be noted here that the buoyancy effects on $\varepsilon$ is neglected by default in FLUENT. The constants used in the standard k- $\varepsilon$ model are: $\mathrm{c}_{1}=1.44, \mathrm{c}_{2}=1.92, \mathrm{c}_{\mu}=0.09, \sigma_{\mathrm{k}}=1.0$, and $\sigma_{\varepsilon}=1.3$

For the temperature distribution calculations, the energy equation can be written as:

$$
\frac{\partial T}{\partial t}+u_{j} \frac{\partial T}{\partial x_{j}}=\frac{\partial}{\partial x_{j}}\left[\left(\frac{v}{\mathrm{Pr}_{L}}+\frac{v_{t}}{\mathrm{Pr}_{T}}\right) \frac{\partial T}{\partial x_{j}}\right]+S_{T}
$$

where $\operatorname{Pr}_{\mathrm{L}}$ is the laminar Prandtl number which is defined by $\operatorname{Pr}_{L}=C_{p} \mu / \kappa$ and $\mathrm{S}_{\mathrm{T}}$ is the source term. 


\subsubsection{RNG model and low-Re RNG model}

The RNG based turbulence model is derived from the instantaneous Navier-Stokes equations, using the Renormalization Group method (RNG) (Yakhot and Orszag, 1986). The mean flow is governed by the incompressible Reynolds averaged continuity and Navier-Stokes equations with an eddy viscosity assumption.

$$
\begin{gathered}
\frac{\partial U_{i}}{\partial x_{i}}=0 \\
\frac{\partial U_{i}}{\partial t}+U_{j} \frac{\partial U_{i}}{\partial x_{j}}=-\frac{1}{\rho} \frac{\partial P}{\partial x_{i}}+\frac{\partial}{\partial x_{j}}\left[\left(v+v_{t}\right)\left(\frac{\partial U_{i}}{\partial x_{j}}+\frac{\partial U_{j}}{\partial x_{x}}\right)\right]-\beta\left(T-T_{r e f}\right) g_{i}
\end{gathered}
$$

The turbulent viscosity $v_{t}$ is solved by a differential equation (3.11).

$$
d\left(\frac{\rho^{2} k}{\sqrt{\varepsilon k}}\right)=1.72 \frac{\hat{v}}{\sqrt{\hat{v}^{3}-1+C_{v}}} d \hat{v}
$$

where $\hat{v}=v_{e f f} / v, C_{v} \approx 100$, and $v_{e f f}=v_{t}+v$.

In the high Re limit, $v_{t}$ tends to

$$
v_{t}=C_{\mu} \frac{k^{2}}{\varepsilon}
$$

where $k=\frac{1}{2} \overline{u_{i}^{\prime} u_{i}^{\prime}}$ and $\varepsilon=v \overline{\frac{\partial u_{i}^{\prime}}{\partial x_{j}} \overline{\frac{\partial u_{i}^{\prime}}{\partial x_{j}}}}$

In this paper, the low_Re RNG turbulence model represents the model using differential equation (3.11) and the RNG turbulence model is represented by equation (3.12). The scale elimination procedure in RNG theory results in a differential equation for turbulent viscosity (Yakhot and Orszag, 1986).

$$
\begin{aligned}
& \frac{\partial k}{\partial t}+U_{j} \frac{\partial k}{\partial x_{j}}=\tau_{i j} \frac{\partial U_{i}}{\partial x_{j}}-\varepsilon+\frac{\partial}{\partial x_{j}}\left[\left(v+\frac{v_{t}}{\sigma_{k}}\right) \frac{\partial k}{\partial x_{j}}\right] \\
& \frac{\partial \varepsilon}{\partial t}+U_{j} \frac{\partial \varepsilon}{\partial x_{j}}=C_{\varepsilon 1} \frac{\varepsilon}{k} \tau_{i j} \frac{\partial U_{i}}{\partial x_{j}}-C_{\varepsilon 2} \frac{\varepsilon^{2}}{k}+\frac{\partial}{\partial x_{j}}\left[\left(v+\frac{v_{t}}{\sigma_{\varepsilon}}\right) \frac{\partial \varepsilon}{\partial x_{j}}\right]
\end{aligned}
$$


The coefficients are $\sigma_{k}=\sigma_{\varepsilon}=0.7179, C_{\mu}=0.085, C_{\varepsilon 1}=1.42 \quad, \quad$ and, $C_{\varepsilon 2}=1.68+\frac{\eta^{3}\left(1-\eta / \eta_{\infty}\right)}{1+\beta \eta^{3}}$, where $\eta=S k / \varepsilon, \eta_{\infty}=4.38, \beta=0.015, S=\left(2 \overline{S_{i j}} \overline{S_{i j}}\right)^{1 / 2}$, and $\overline{S_{i j}}$

is the mean rate of strain tensor. The RNG model has the same form as the standard k- $\varepsilon$ turbulence model, except $C_{\mu}=0.09$ is used by the latter. The model parameters in the $\varepsilon$ equation are also different from those for the standard k- $\varepsilon$ model. Besides the difference in the model parameters, another major difference between $\mathrm{RNG}$ and standard $\mathrm{k}-\varepsilon$ is that there is an additional term in the $\varepsilon$ equation for the RNG turbulence model, which accounts for the effect of the rapid strain. The RNG model is known to be more responsive to the effects of rapid strain and streamline curvature than the standard k- $\varepsilon$ model, which explains the better performance of the RNG model for certain classes of flows.

\subsubsection{RSM model}

The Reynolds stress model involves calculation of the individual Reynolds stresses, $\overline{u_{i}^{\prime} u_{j}^{\prime}}$, using differential transport equations. The individual Reynolds stresses are then used to obtain closure of the Reynolds-averaged momentum equation. The exact transport equations for the transport of the Reynolds stresses, $\overline{u_{i}^{\prime} u_{j}^{\prime}}$, may be written as follows:

$$
\begin{aligned}
& \underbrace{\frac{\partial\left(\rho \overline{u_{j}{ }^{\prime} u_{i}{ }^{\prime}}\right)}{\partial t}}_{\text {Local time derivative }}+\underbrace{\frac{\partial\left(\rho U_{k} \overline{u_{i}{ }^{\prime} u_{j}{ }^{\prime}}\right)}{\partial x_{k}}}_{C_{i j} \equiv \text { Convection }}=\underbrace{-\rho\left(\overline{u_{j}{ }^{\prime} u^{\prime}{ }_{k}} \frac{\partial U_{i}}{\partial x_{k}}+\overline{u_{i}{ }^{\prime} u_{k}^{\prime}} \frac{\partial U_{j}}{\partial x_{k}}\right)}_{P_{i j} \equiv \text { production }}-\underbrace{\frac{\partial\left(\rho \overline{u_{i}{ }^{\prime} u_{j}{ }^{\prime} u_{k}{ }_{k}}\right)}{\partial x_{k}}}_{T_{i j} \equiv \text { Eurbulent diffusion }} \\
& -\underbrace{\left[\overline{u_{i}{ }^{\prime} \frac{\partial p^{\prime}}{\partial x_{j}}}+\overline{u_{j}{ }^{\prime} \frac{\partial p^{\prime}}{\partial x_{i}}}\right]}_{R_{i j} \equiv \text { Redistribution }}-\underbrace{2 \mu \overline{\frac{\partial u_{i}{ }^{\prime}}{\partial x_{k}} \frac{\partial u_{j}{ }^{\prime}}{\partial x_{k}}}}_{\varepsilon_{i j} \equiv \text { Dissipation }}+\underbrace{\frac{\partial}{\partial x_{k}} \mu\left(\frac{\partial \overline{u_{i}{ }^{\prime} u_{j}{ }^{\prime}}}{\partial x_{k}}\right)}_{L_{i j} \equiv \text { Molecule diffusion }}-\underbrace{\rho \beta\left(g_{i} \overline{u_{j}{ }^{\prime} \theta}+g_{j} \overline{u_{i}{ }^{\prime} \theta}\right)}_{G_{i j} \equiv \text { Buoyancy production }}
\end{aligned}
$$

Of the various terms in these exact equations, $\mathrm{C}_{\mathrm{ij}}, \mathrm{P}_{\mathrm{ij}}$, and $\mathrm{L}_{\mathrm{ij}}$ do not require any modeling. However, $T_{i j}, R_{i j}, \varepsilon_{i j}$, and $G_{i j}$ need to be modeled to close the equations.

The turbulent diffusion is modeled by using a scalar turbulent diffusivity as follows, 


$$
T_{i j}=\frac{\partial}{\partial x_{k}}\left(\frac{\mu_{t}}{\sigma_{k}} \frac{\partial \overline{u_{i}^{\prime} u_{j}^{\prime}}}{\partial x_{k}}\right)
$$

Lien and Leschziner (1994) derived a value of $\sigma_{\mathrm{k}}=0.82$ by applying the diffusion model to the case of a planar homogeneous shear flow. Note that this value of $\sigma_{\mathrm{k}}$ is different from that in the standard k- $\varepsilon$ models, in which $\sigma_{\mathrm{k}}=1.0$ is commonly used.

The pressure-strain term is modeled according to the proposals by Gibson and Launder (1978). The reader is referred to their paper for detailed information on the modeling of the pressure-strain term. The production terms due to buoyancy are modeled as

$$
G_{i j}=\beta \frac{\mu_{t}}{\operatorname{Pr}_{t}}\left(g_{i} \frac{\partial T}{\partial x_{j}}+g_{j} \frac{\partial T}{\partial x_{i}}\right)
$$

where $\operatorname{Pr}_{t}$ is the turbulent Prandtl number for energy, with a default value of 0.85 .

In general, when the turbulence kinetic energy is needed for modeling a specific term, it is obtained by taking the trace of the Reynolds stress tensor:

$$
k=\frac{1}{2} \overline{u_{i}^{\prime} u_{i}^{\prime}}
$$

The dissipation tensor, $\varepsilon_{\mathrm{ij}}$ is modeled as

$$
\varepsilon_{i j}=\frac{2}{3} \delta_{i j} \rho \varepsilon
$$

The scalar dissipation rate, $\varepsilon$, is computed from a model transport equation similar to that used in the

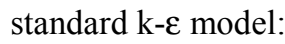

$$
\frac{\partial}{\partial t}(\rho \varepsilon)+\frac{\partial}{\partial x_{i}}\left(\rho \varepsilon u_{i}\right)=\frac{\partial}{\partial x_{i}}\left[\left(\mu+\mu_{t}\right) \frac{\partial \varepsilon}{\partial x_{j}}\right]+C_{\varepsilon 1} \frac{1}{2}\left[P_{i i}+C_{\varepsilon 3} G_{i i}\right] \frac{\varepsilon}{k}-C_{\varepsilon 2} \rho \frac{\varepsilon^{2}}{k}
$$

where $\sigma_{k}=1.0, \mathrm{C}_{\varepsilon 1}=1.44, \mathrm{C}_{\varepsilon 2}=1.92, \mathrm{C}_{\varepsilon 3}$ is evaluated as a function of the local direction relative to the gravitational vector,

$$
C_{\varepsilon 3}=\tanh \left|\frac{v}{u}\right|
$$


where $\mathrm{v}$ is the component of the flow velocity parallel to the gravitational vector and $\mathrm{u}$ is the component of the flow velocity perpendicular to the gravitational vector. In this way, $\mathrm{C}_{\varepsilon 3}$ will become zero. The turbulent viscosity, $\mu_{t}$, is computed in a similar manner to the k- $\varepsilon$ models (Eq. 3.3).

Whenever flow enters the domain, the individual Reynolds stresses, $\overline{u_{i}^{\prime} u_{j}^{\prime}}$ and the turbulence dissipation rate, $\varepsilon$, are required as inlet conditions. These quantities can be derived from the turbulence intensity and characteristic length for the current case, as follows:

$$
\begin{array}{ll}
\overline{u_{i}^{\prime 2}}=\frac{2}{3} k & (i=1,2,3) \\
\overline{u_{i}^{\prime} u_{j}^{\prime}}=0 & (i \neq j) \\
\varepsilon=C_{\mu}^{3 / 4} \frac{k^{3 / 2}}{l} &
\end{array}
$$

where 1 is the turbulence length scale and is determined from the relationship with the hydraulic diameter $\mathrm{D}_{\mathrm{H}}$ :

$$
l=0.07 D_{H}
$$

\subsubsection{LES simulation}

Turbulent flows are characterized by eddies with a wide range of length and time scales. The largest eddies are typically comparable in size to the characteristic length of the mean flow. The smallest scales are responsible for the dissipation of turbulence kinetic energy.

In LES, large eddies are resolved directly, while small eddies are modeled. Large eddy simulation (LES) thus falls between DNS and RANS in terms of the fraction of the resolved scales. The rationale behind LES can be summarized as follows:

- $\quad$ Momentum, mass, energy, and other passive scalars are transported mostly by large eddies.

- $\quad$ Large eddies are more problem-dependent. They are dictated by the geometries and boundary conditions of the flow involved. 
- $\quad$ Small eddies are less dependent on the geometry, tend to be more isotropic, and are consequently more universal.

- $\quad$ The chance of finding a universal turbulence model is much higher for small eddies.

The governing equations employed for LES are obtained by filtering the time-dependent NavierStokes equations in either Fourier (wave-number) space or configuration (physical) space. The filtering process effectively filters out the eddies whose scales are smaller than the filter width or grid spacing used in the computations. The resulting equations thus govern the dynamics of large eddies.

A filtered variable (denoted by an overbar) is defined by

$$
\bar{\phi}(x)=\int_{D} \phi\left(x^{\prime}\right) G\left(x, x^{\prime}\right) d x^{\prime}
$$

where $\mathrm{D}$ is the fluid domain, and $\mathrm{G}$ is the filter function that determines the scale of the resolved eddies.

In FLUENT, the finite-volume discretization itself implicitly provides the filtering operation:

$$
\bar{\phi}(x)=\frac{1}{V} \int_{D} \phi\left(x^{\prime}\right) d x^{\prime}, \quad x^{\prime} \in V
$$

where $\mathrm{V}$ is the volume of a computational cell. The filter function, $\mathrm{G}$, implied here is then

$$
G\left(x, x^{\prime}\right)= \begin{cases}1 / V & x^{\prime} \in V \\ 0 & x^{\prime} \text { otherwise }\end{cases}
$$

Filtering the Navier-Stokes equations, one obtains

$$
\begin{gathered}
\frac{\partial \rho}{\partial t}+\frac{\partial}{\partial x_{i}}\left(\rho \overline{u_{i}}\right)=0 \\
\frac{\partial}{\partial t}\left(\rho \overline{u_{i}}\right)+\frac{\partial}{\partial x_{j}}\left(\rho \overline{u_{i} u_{j}}\right)=\frac{\partial}{\partial x_{j}}\left[\mu\left(\frac{\partial \overline{u_{i}}}{\partial x_{j}}+\frac{\partial \overline{u_{j}}}{\partial x_{i}}\right)\right]-\frac{\partial \bar{p}}{\partial x_{i}}-\frac{\partial \tau_{i j}}{\partial x_{j}}
\end{gathered}
$$

and $\tau_{i j}$ is the subgrid-scale stress defined by

$$
\tau_{i j}=\rho \overline{u_{i} u_{j}}-\rho\left(\overline{u_{i}}\right)\left(\overline{u_{j}}\right)
$$




\section{Subgrid Scale Model}

The subgrid-scale stresses resulting from the filtering operation are unknown, and thus require modeling. The subgrid-scale turbulence model used in this study employs the Boussinesq hypothesis as in the RANS models, computing subgrid-scale turbulent stresses from

$$
\tau_{i j}-\frac{1}{3} \tau_{k k} \delta_{i j}=-2 \mu_{t} \overline{S_{i j}}
$$

where $\mu_{t}$ is the subgrid-scale turbulent viscosity, and $\overline{S_{i j}}$ is the rate-of-strain tensor for the resolved scale defined by

$$
\overline{S_{i j}}=\frac{1}{2}\left(\frac{\partial \overline{u_{i}}}{\partial x_{j}}+\frac{\partial \overline{u_{j}}}{\partial x_{i}}\right)
$$

The subgrid model used in this study is Smagorinsky-Lilly model which was first proposed by Smagorinsky (1963). In this model, the eddy viscosity is modeled by

$$
\mu_{t}=\rho L_{s}^{2}|\bar{S}|
$$

where Ls is the mixing length for subgrid scales and

$$
|\bar{S}| \equiv \sqrt{2 \overline{S_{i j} \overline{S_{i j}}}}
$$

Ls is computed using

$$
L_{s} \equiv \min \left(\kappa d, C_{s} V^{1 / 3}\right)
$$

where $\kappa$ is the von Karman constant, $d$ is the distance to the closest wall, Cs is the Smagorinsky constant, and $\mathrm{V}$ is the volume of the computational cell. Lilly (1966) derived a value of 0.17 for Cs for homogeneous isotropic turbulence in the inertial subrange. However, this value was found to cause excessive damping of large-scale fluctuations in the presence of mean shear and in transitional flow as when near a solid boundary, and has to be reduced in such regions. In short, Cs is not a universal constant, which is the most 
serious shortcoming of this simple model. Nonetheless, a Cs value of around 0.1 has been found to yield the best results for a wide range flows, and is the default value in FLUENT.

These three dimensional equations are solved using a segregated grid approach with pressure projection. The well known QUICK scheme (Leonard, 1979) is used for all the convective terms, and a central differencing scheme is used for the diffusion terms. Two types of boundary conditions are applied to the system under study, namely the wall boundary conditions and the entry-exit conditions. All of the solid walls in this investigation are at rest. Therefore, both the mean and fluctuating velocities of the fluid at these boundaries are identically zero for the turbulent flow, as there is no slip and no mass-transport at the walls. In addition, wall functions are used to model the near wall region with an empirical logarithmic velocity profile in terms of the distance from the wall. The inlet boundary condition is set as a specified velocity inlet, whereas the exit (outlet) boundary condition is set as a specified pressure outlet.

\subsubsection{Eulerian scalar transport}

If a species (acetone vapor in the present study) is added to the system, the mean concentration can be calculated by making use of the following transport equation:

$$
\frac{\partial C}{\partial t}+\frac{\partial}{\partial x_{i}}\left[u_{i} C-\left(\frac{v}{S c_{L}}+\frac{v_{t}}{S c_{T}}\right) \frac{\partial C}{\partial x_{i}}\right]=S_{\phi}
$$

Here, $\mathrm{Sc}_{\mathrm{L}}$ is the laminar $\mathrm{Schmidt}$ number and $\mathrm{Sc}_{\mathrm{T}}$ is the turbulent Schmidt number. The mass flow rate of the scalar is $\dot{m}=1.0 \mathrm{e}-4 \mathrm{~kg} / \mathrm{s}$. The volume $\mathrm{V}$ of the source region is $\sim 0.005 \mathrm{~m}^{3}$. The source term $\mathrm{S}_{\phi}$ is set to $\dot{m} / \forall \mathrm{kg} /\left(\mathrm{m}^{3} \cdot \mathrm{s}\right)$. The source region is $1.2 \mathrm{~m}$ above the ground and $0.5 \mathrm{~m}$ downstream of the human body. The values of $\mathrm{Sc}_{\mathrm{L}}=1.42$ (Mills, 1999) and $\mathrm{Sc}_{\mathrm{T}}=1.30$ are selected for the purpose of this study.

\subsubsection{Lagrangian trajectory tracking}

Although gaseous vapor is used in this study, the Lagrangian model is investigated because it can be easily adapted in the simulation of aerosols. For the Lagrangian simulation of gaseous contaminants, each of the parcels represents a certain amount of acetone vapor and closely follows the air flow. These fictitious particles are released from the plane shown in Figure 3.1. In order to conform to the Eulerian 
scalar transport method, the flow rate, and the diameter of the representative particles are set equal to 0.1 $\mathrm{g} / \mathrm{s}$, and $1 \mu \mathrm{m}$, respectively.

Modeling gases with $1 \mu \mathrm{m}$ aerosols presents negligible errors because they follow airflows so faithfully, as is demonstrated elsewhere mathematically (Hjelmfelt and Mockros, 1966). Indeed, one of the most important laboratory measurement devices for velocity and turbulence, Particle Image Velocimetry, uses much larger aerosols (up to $50 \mu \mathrm{m}$, Saga et al., 2000) to track the fluid motions.

Also, it has to be mentioned that the concentration levels for the Eulerian method are calculated at the cell center for that particular cell, and the concentration levels using the Lagrangian method are calculated by dividing the cumulative trajectory mass by the volume of that particular cell they are residing in. In both methods one would get a single value for that particular cell.

The results are presented and discussed in two parts. First, the grid convergence and iterative convergence are investigated, which is followed by the predictions of the impact of critical factors which includes the body shape, the transport model, the turbulence model, the ventilation intensity, the free stream turbulence and the heat released from the body. The second part will be the main focus of this study.

\subsection{Grid convergence and iterative convergence}

The five different numerical grid distributions listed in Table 3.1 were used to assess the grid dependency of the RANS results for the sharp body simulations. The streamwise velocity component (velocity component in $\mathrm{x}$ direction as shown in Figure 3.1), turbulent kinetic energy (k), and concentration distributions (with Eulerian method) along four different vertical lines on the center-plane for all grid distributions are shown in Figure 3.5, where d represents the downstream distance of the vertical line measured from the body. It can be seen that mesh \#1 and mesh \#2 are so coarse that the turbulent kinetic energy profiles predicted with them in the downstream of the leg are much higher than the ones with other meshes. With mesh \#3, \#4 and \#5, the results are very close in the downstream of the upper body, which is believed to be the most important region for the worker exposure. It has been mentioned earlier that mesh \#3 is refined just in the region around the body based on mesh \#2 and it has less cells than mesh \#4. However, the turbulent kinetic energy with mesh \#3 is even closer to the result with mesh \#5 (the finest 
grid) at some regions. Since running a case with mesh \#3 takes less time than with mesh \#4, it has been used to study the effect of body shapes and transport methods.

The values of turbulent kinetic energy and concentration for the sharp body with mesh \#2, \#4 and $\# 5$ are listed in Table 3.2, where $\mathrm{h}$ is the averaged grid size calculated as

$$
h=\sqrt[3]{\frac{\text { volume }}{\text { number of cells }}}
$$

and $\mathrm{h} 5$ is the finest average grid size. Convective transport due to recirculation regions formed in the wake of the body and the diffusive nature of the gaseous contaminants causes a nonzero concentration field upstream of the source. Using Richardson extrapolation on the concentration data which shows monotonic convergence, the observed order of the numerical schemes can be calculated which is consistent with the initial arrangement (QUICK for convection terms and central differencing for diffusion terms). The extrapolated "exact" concentration was $0.00489 \mathrm{~kg} / \mathrm{m}^{3}$. The grid convergence index of concentration at the breathing zone for mesh \#2 is approximately 11\% (Extrapolated Relative Error is about $9 \%$ ). For the turbulent kinetic energy data at the breathing zone, non-monotonic convergence was shown since $\mathrm{k}(\# 5)<$ $\mathrm{k}(\# 2)<\mathrm{k}(\# 4)$ while $\mathrm{h} 5<\mathrm{h} 4<\mathrm{h} 2$. The relative error of $\mathrm{k}$ for mesh $\# 2$ was $8 \%$ based on mesh \#5.

The concentrations at the breathing zone (mouth) using the Eulerian method are compared in Figure 3.6, where $\mathrm{h} 1$ is the coarsest average grid size. Figure 3.6 shows that the lowest exposure is predicted for the simulations with the rounded body. The concentrations do not asymptotically approach to some value as expected. Instead, the cells composing the source region change with the mesh itself, which affect the grid convergence in terms of concentration as a function of the source position and source size (Kulmala et al., 1996). Additional setup effort was needed to keep the source region exactly the same for each mesh. On the other hand, for the purpose of this study, it is shown clearly that the body shape has significant influence on the predicted worker exposure. 

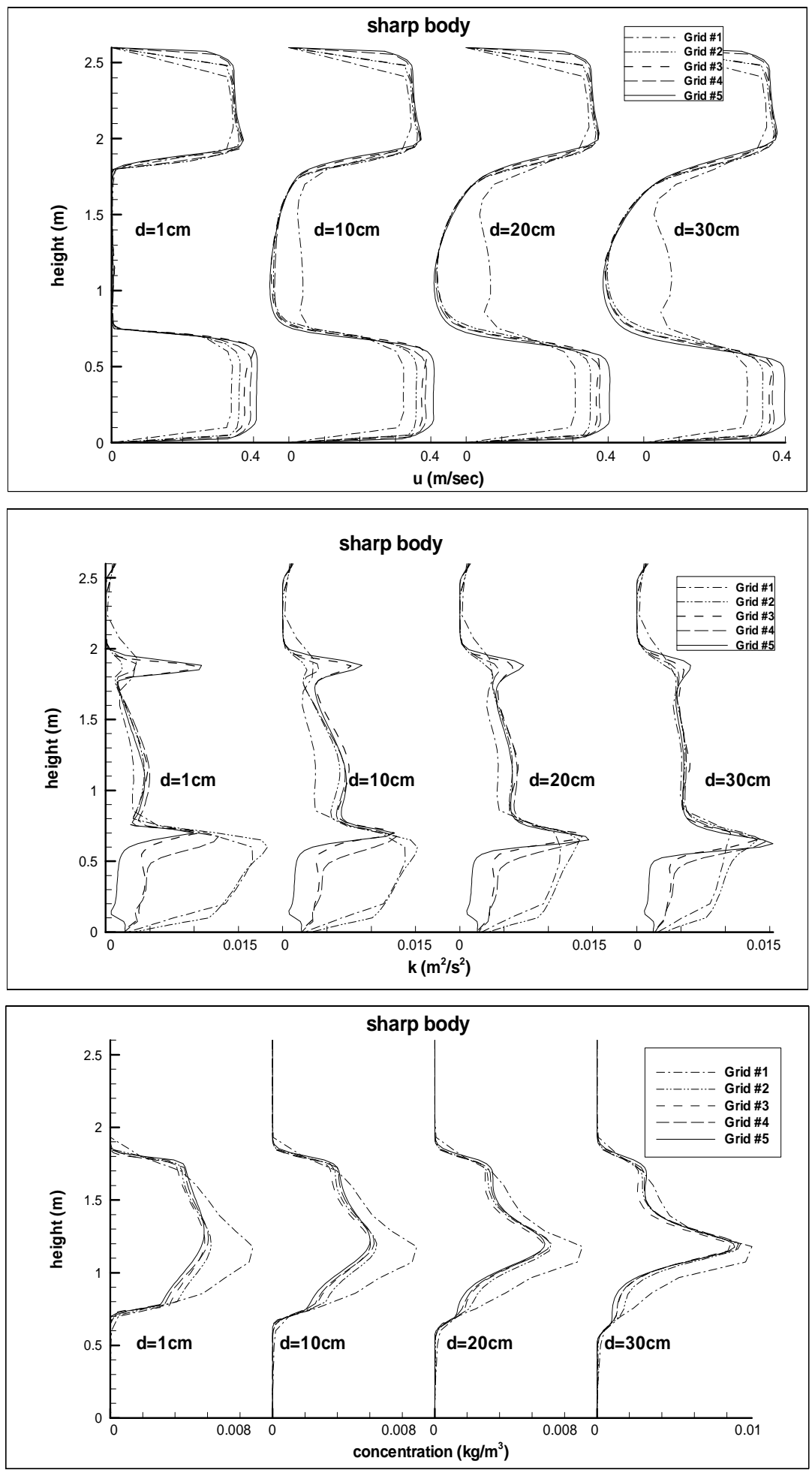

Figure 3-5 Streamwise velocities, turbulent kinetic energy, and concentrations at several vertical lines downstream of the body on the central plane with different grid distributions for the sharp body 
Table 3.2 Turbulent kinetic energy and concentration at the breathing zone for the sharp body

\begin{tabular}{|c|c|c|c|}
\hline & Grid \#5 & Grid \#4 & Grid \#2 \\
\hline $\mathrm{h} / \mathrm{h} 5$ & 1.0 & 1.465 & 2.08 \\
\hline $\mathrm{k}\left(\mathrm{m}^{2} / \mathrm{s}^{2}\right)$ & 0.001799 & 0.001939 & 0.001814 \\
\hline Concentration $\left(\mathrm{kg} / \mathrm{m}^{3}\right)$ & 0.00482 & 0.00472 & 0.00448 \\
\hline
\end{tabular}

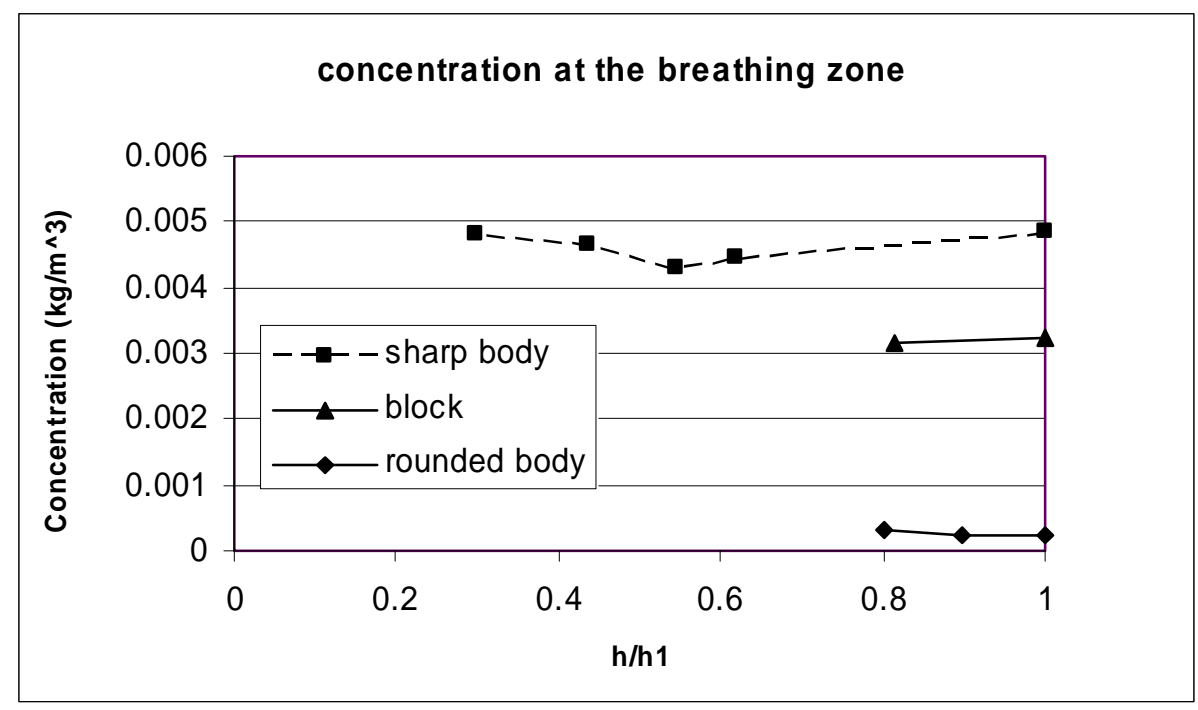

Figure 3-6 Concentrations at the breathing zone with different grid distributions and different body shapes

There was no distinguishable difference between the results at a residual criterion of 0.001 and of 0.0001 , so a residual of 0.0001 used in this study should achieve a good convergence in terms of iteration convergence. The streamwise velocity component at the mouth also was monitored in the calculation to check if it approached an asymptotic value when the assigned residual was reached, in order to avoid false convergence by using a fixed value of residual.

\subsection{Influence of critical factors on worker exposure}

\subsubsection{Effect of the body shape}

Comparison of the flow-field predictions with the block, the sharp, and the rounded body indicated that the predicted flow-field with the block has one large recirculation region, whereas the sharp and rounded bodies each induced two smaller recirculation regions (Figure 3.7). The recirculation zone 
around the head suggests that contaminants trapped in that region are more likely to be inhaled. Moreover, the contaminants accumulated in the waist region could be transferred to the breathing zone from the lower recirculation zone. However, the flow around the sharp and rounded bodies shows different separation profiles, which is an important issue. For the sharp body, the flow separates at the corner of the head, whereas with the rounded body, the air flows along the forehead and separates from the body near the eye level. This means that such small issues as the hairstyle, the size and shape of the hat worn, and the angle of the forehead could affect the exposure levels. Another important issue is the clothes. If the worker wears an apron, the shape will be more like a sharp body since the apron blocks the flow between legs. This indicates that the clothes the worker wear may significantly change the flow field and the subsequent exposure levels.

Using the Eulerian method for each body shape, the predicted non-dimensionalized concentration levels of the contaminant gas at various locations were compared for trend analysis (see Figure 3.8). It is clear that the block body cannot be used to represent a worker accurately. With the sharp and rounded body, the concentration level at the chest region is higher than that at the breathing zone $\left(\mathrm{C}_{\text {chest }} / \mathrm{C}_{\text {nose }}\right.$ is 13.6 for the rounded body). This observation qualitatively agrees with experimental results (Guffey et al., 2001) which concluded the concentrations at the chest averaged about 2.9 times the concentrations at the nose and the ratio decreased significantly with increasing velocities $\left(\mathrm{C}_{\text {chest }} / \mathrm{C}_{\text {nose }}=2.4\right.$ at $\left.\mathrm{V}_{\text {inlet }}=0.24 \mathrm{~m} / \mathrm{s}\right)$. It should be noted that these experimental conditions were different from the current study in some aspects. For instance, $\mathrm{SF}_{6}$ was used in the experiments and the geometric average of the concentrations at three points (left chest, middle chest and right chest) was taken to represent the concentration in the chest area. In addition, the manikin shape and the source size in the experiments are also different. These differences may contribute to the different ratios of $\mathrm{C}_{\text {chest }}$ to $\mathrm{C}_{\text {nose }}$ found in the calculations and the experiments. However, the same trend observed by both the calculations and the experiments suggests that it may not be a good practice to measure the concentrations around the chest area as surrogates for the inhaled concentrations, as is current practice. With the block figure the concentration ratio of chest to nose is reversed, which illustrates that the trend could be inaccurately predicted with crude representatives of the human body. 


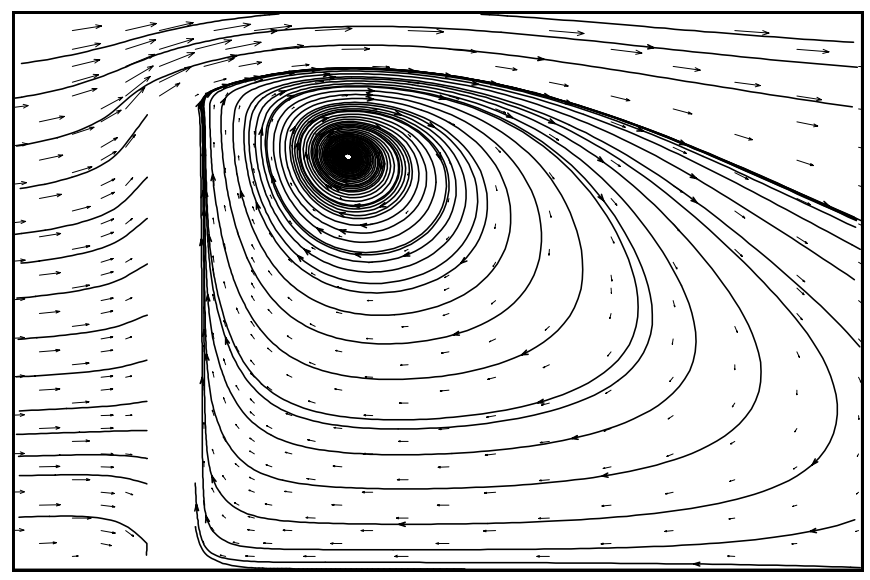

a) block

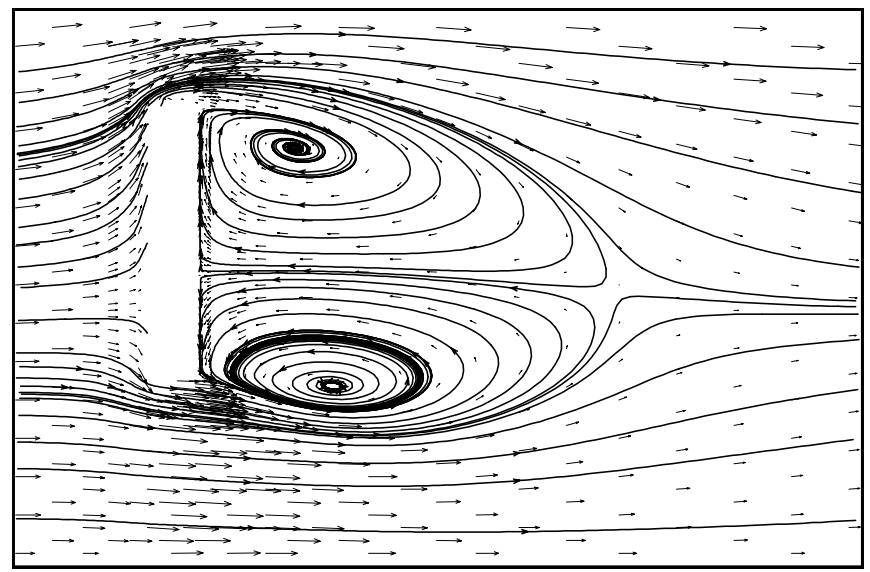

b) sharp body

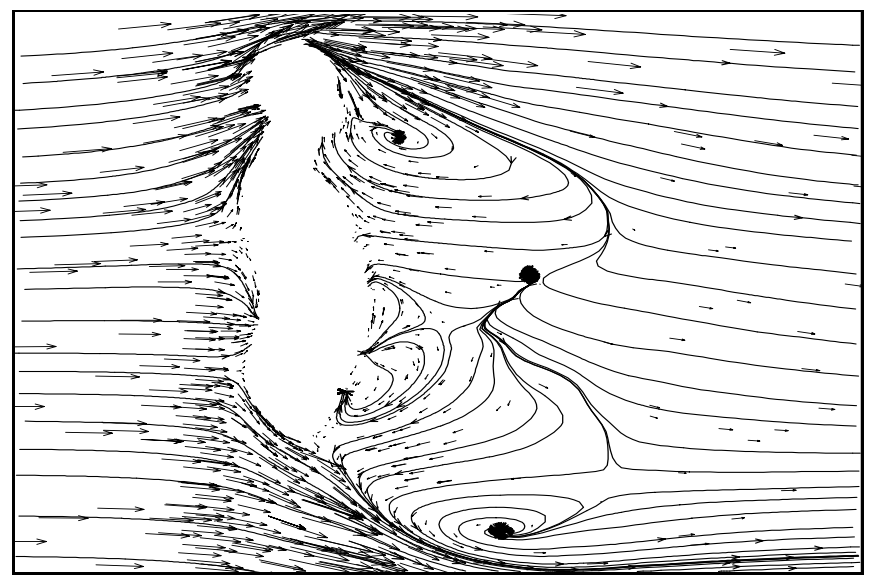

(c) rounded body

Figure 3-7 Comparison of the flow field with a block, a sharp body and a rounded body - velocity vectors and streamlines on the center plane (side elevation) 


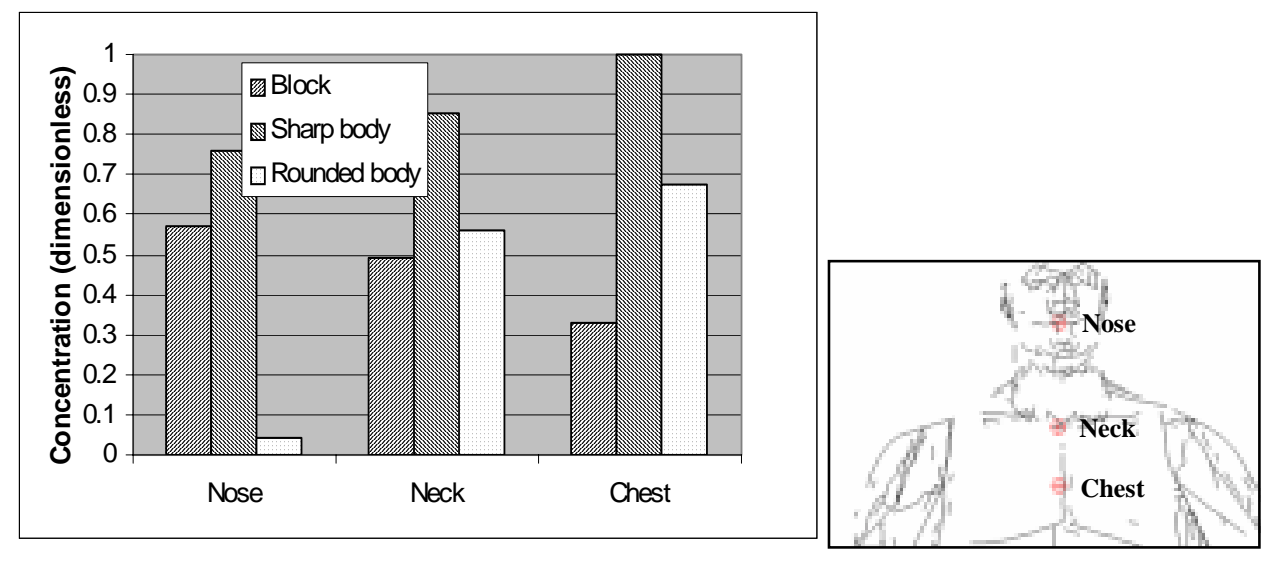

Figure 3-8 Comparison of normalized concentration at different points $1.0 \mathrm{~cm}$ downstream of human body for different body shapes (Normalized with the highest concentration value)

Figure 3.9 presents the predicted concentration distributions $1 \mathrm{~cm}$ downstream of the body obtained from the simulations using the Eulerian scalar transport method. It is seen that the contaminants are accumulated at the top of the block, whereas the contaminants for the sharp and rounded body are diffused over a much wider region of the body accumulating mostly in the chest region. This again shows that using less accurate shapes for the human body may lead to inaccurate results. 

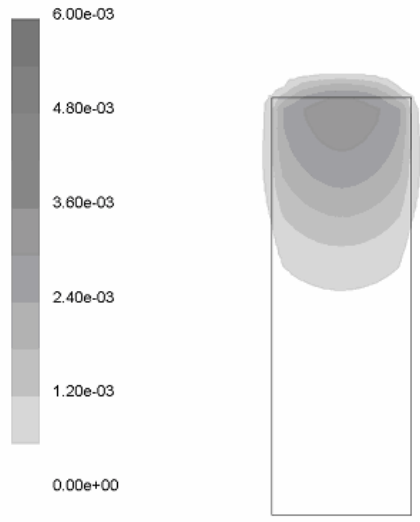

(a) block
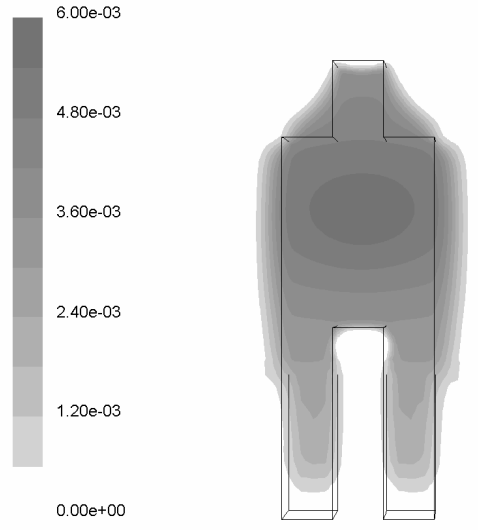

(b) sharp body
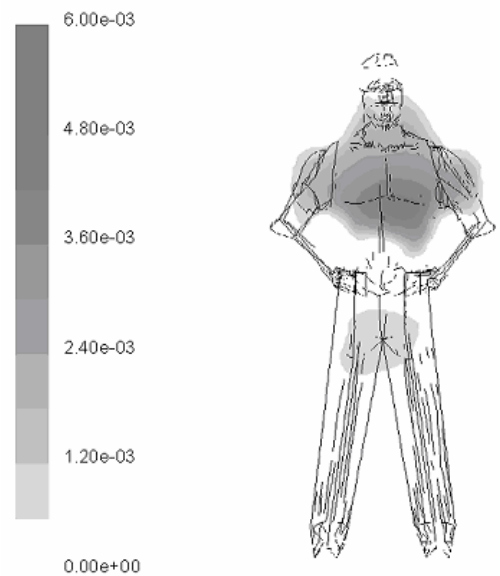

(c) rounded body

Figure 3-9 Concentration distribution; Eulerian method, on a cross-section $1.0 \mathrm{~cm}$ downstream of the body 


\subsubsection{Effect of the contaminants transport model}

Using the Lagrangian method, the concentration at a plane $1.0 \mathrm{~cm}$ downstream of the body is shown in Figure 3.10d. Compared to the results of the Eulerian method in Figure 3.9b, it can be argued that both methods predict the concentration levels on the same order, although the Lagrangian method shows much more randomness, which is directly associated with the method itself since a random velocity component is added to the mean fluid velocity to simulate trajectory dispersion due to turbulence. It has been seen during the simulations that the number of trajectories tracked in the Lagrangian simulations is also an important factor affecting the predicted concentration levels. The more trajectories tracked, the smoother the concentration contours become (see Figures 3.11c and 3.11e), since a better averaging over a volume can be applied to calculate the concentration levels.

The symmetry-plane concentration contours are shown in Figures 3.11a, 3.11b and 3.11c for both the Eulerian method and the Lagrangian method. The latter was repeated for different numbers of representative particle trajectories. Here again, the Eulerian method exhibits a more diffusive concentration field than the Lagrangian method. The latter shows a higher degree of irregularity because of the applied discrete random walk tracking model used in the FLUENT code. Nevertheless, the predictions of the two methods are in fairly good agreement with each other (see also Figure 3.11).

The local concentrations at different locations $1.0 \mathrm{~cm}$ downstream of the sharp body are compared for both the Eulerian and Lagrangian predictions in Figure 3.11. The values are normalized by dividing by the highest concentration value $\left(\mathrm{C}_{\max }=0.00484 \mathrm{~kg} / \mathrm{m}^{3}\right)$ that was predicted for both simulations. This was done since the trend of the concentrations is the main concern of this study, not the absolute values of the concentrations. The Eulerian method predicts a more uniform concentration when compared to the predictions obtained via the Lagrangian method. Although it is true that tracking more trajectories creates a more uniform concentration field for the Lagrangian method, it has to be noted that it is very computationally expensive to use a large number of trajectories. It is noteworthy to mention that both methods tend to predict higher concentrations near the lapel compared to the mouth/nose level. 


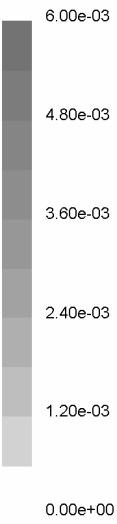

(a) Eulerian model (side view)
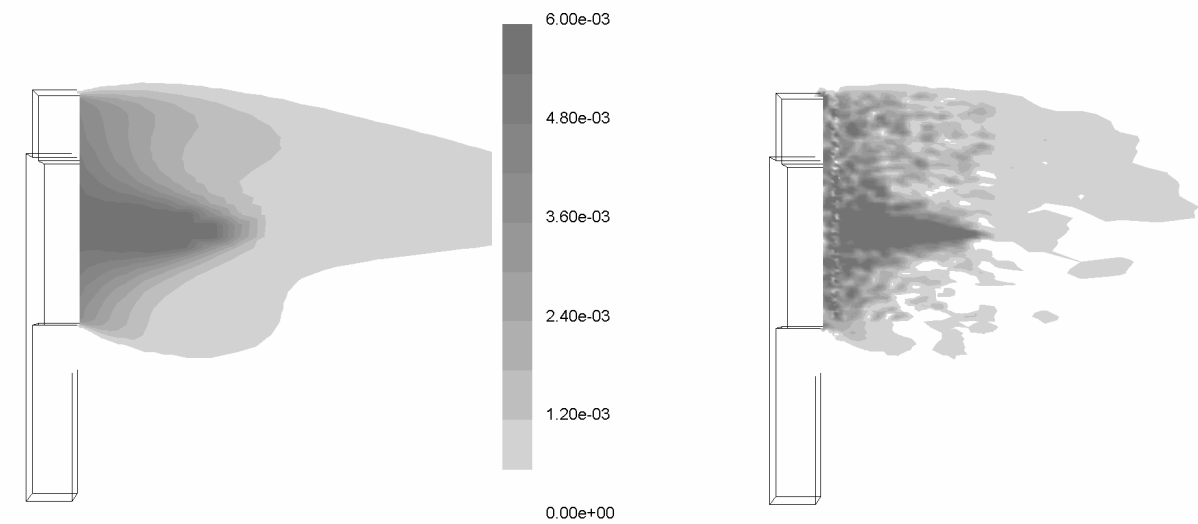

(b) Lagrangian model (3,480 trajectories) (side view)

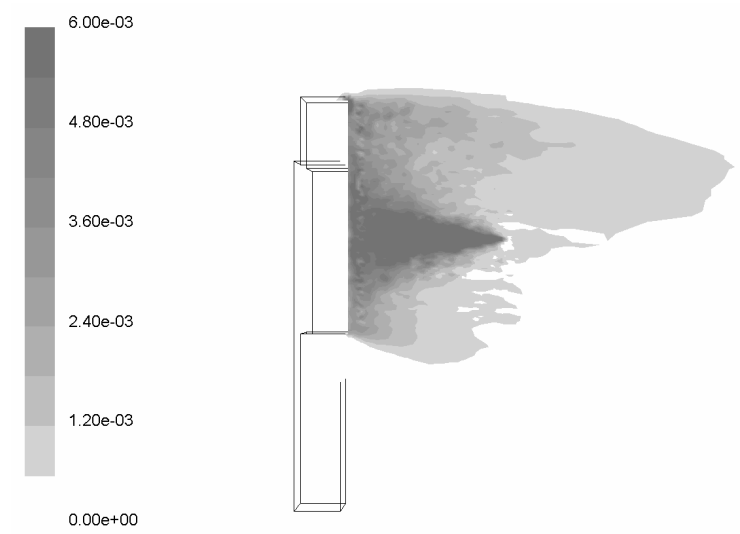

(c) Lagrangian model (35,100 trajectories) (side view)
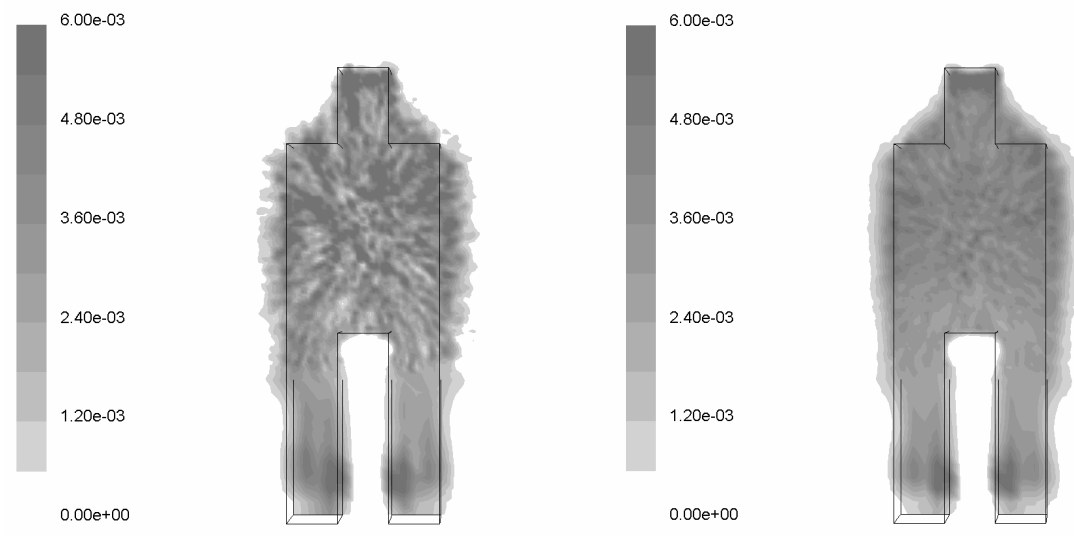

(d) Lagrangian model (3,480 trajectories)

(e) Lagrangian model (35,100 trajectories)

Figure 3-10 Concentration contours a,b,c) on the center plane d,e) on a cross section $1.0 \mathrm{~cm}$ downstream of the body 

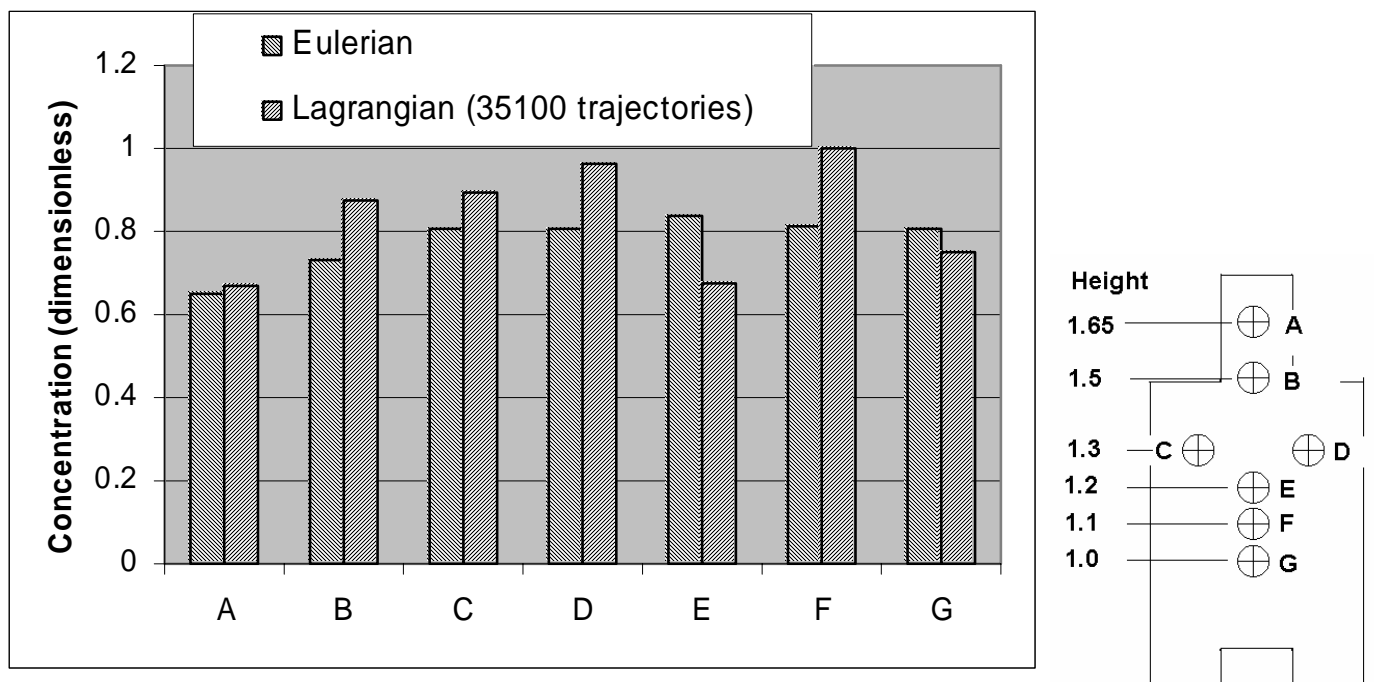

Figure 3-11 Normalized concentrations at different locations at a cross section $1 \mathrm{~cm}$ downstream of the sharp body

\subsubsection{Variation of turbulence models}

Turbulence levels and turbulence length scales associated with the turbulent eddies are probably one of the most important factors that can influence the dispersion of aerosol particulate. Hence, it is essential that turbulence models used are capable of accurately predicting the major characteristics of the turbulent flows in the working environment. Simulations were performed with four different models,

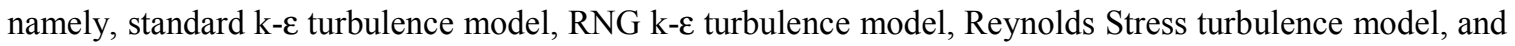
Large Eddy Simulation (LES). The results from each are compared to the predicted concentration levels at critical locations in the vicinity of the face of the simulated manikin.

The governing equations solved are the well-known Reynolds Averaged Navier-Stokes equations (RANS) and the equation of continuity. In the RANS approach the turbulent stresses resulting from the time averaging of non-linear terms need to be calculated with an appropriate turbulence model, hence the use of the turbulence models mentioned above.

The governing equations employed for LES are obtained by filtering the time-dependent NavierStokes equations in physical space. The filtering process effectively filters out eddies whose scales are smaller than roughly the computational cell size. The resulting equations governing the dynamics of large eddies are very similar to the RANS equations, but in this case, the turbulent stresses are replaced by 
subgrid stresses. The Smagorinsky-Lilly subgrid-scale model (Lilly, 1966) is employed to solve the subgrid stresses in the present application. For values of the model constants and detailed information about these models, the reader is referred to the Fluent manual.

Constant velocity inlet and pressure outlet boundary conditions were used. The inlet velocity was taken as $0.3 \mathrm{~m} / \mathrm{s}$ with a turbulence intensity of $0.3 \%$. These values were rough estimates to the experimental conditions and they are also representative of a usual working environment (Baldwin and Maynard, 1998). The characteristic length scale was chosen to be $0.01 \mathrm{~m}$, which represented the turbulence generating grid size at the inlet of the tunnel. All of the solid walls in this investigation were at rest; therefore, both the mean and fluctuating velocities of the fluid at these boundaries were identically zero for the turbulent flow, as there was no slip and no mass-transport at the walls. Standard wall-function boundary condition was applied at the grid nodes nearest to the walls. Tracer gas was released from an inert surface, which was approximated by a $0.3 \mathrm{~m} \times 0.3 \mathrm{~m}$ square and located $1.0 \mathrm{~m}$ high from the floor and $0.25 \mathrm{~m}$ downstream from the body. For the present study, the unheated body is considered.

In the present application the source term $S$ is set to $0.0216 \times p$. The volume of this region is $0.00463 \mathrm{~m}^{3}$, such that the mass flow rate of the scalar is $0.0001 \mathrm{~kg} / \mathrm{s}$. The values of $\mathrm{Sc}_{\mathrm{L}}=0.32$ and $\mathrm{Sc}_{\mathrm{T}}=$ 0.70 in Eq. 3.38 (Lan and Viswanathan, 2001) are selected for the purpose of this study.

\section{Results}

The streamlines colored by y-velocity magnitude on the center-plane are shown in Figure 3-12(a) for the case with standard k- $\varepsilon$ turbulence model. As the plane slices the human-body in the center, the flow between the legs also can be seen in this figure. It created a re-circulation region around the waist. The contaminants are usually trapped within such recirculation zones if turbulent diffusion is not dominant. It should be noted that if the worker wears an apron, the exposure he/she would receive could be quite different from the one observed in this study.

It can be seen from Figure 3.12 that the convective flow will bring the contaminant upward from the waist region. This flow is partly due to buoyancy as it is seen in Figure 3.13. This suggests that the heat flux from the human body could contribute to the worker exposure. Figure 3.12 shows that air flows along 
the forehead and separates from the body near the eye level. This means that such small issues as the hairstyle, the size and shape of the hat worn, and the angle of the forehead could affect the exposure levels.

Figure 3.12 further shows that the overall quantitative features of the flow patterns seen in the vicinity of the body are very similar for the RANS simulations regardless of the turbulence model used. There are big differences in the predicted turbulent kinetic energy profiles (see Fig. 3.14) when different turbulence models are used. The LES results (Fig. 3.12d), which were averaged over a three minute interval, do depict significantly different flow patterns compared to RANS results. This leads to species concentration prediction as observed in Figure 3.15.

The turbulent kinetic energy and the tracer gas concentration determined with the Eulerian method are depicted in Figure 3.13 and 3.14 for different turbulence models at different horizontal distances from the mouth along a vertical line in the middle plane. The standard k- $\varepsilon$ turbulence model exhibited much higher turbulence kinetic energy levels than RNG turbulence model and RSM. This usually leads to a more diffusive concentration field. The turbulent kinetic energy profiles obtained from the RNG turbulence model and RSM are very similar. The subgrid turbulent kinetic energy, Ksgs, presented in Figure 3.14 indicates that most of the energetic large eddies are captured by the LES technique. Note that Ksgs represents the unresolved part of the turbulent kinetic energy by LES. This is apparent from the low levels of Ksgs compared to K obtained from RANS. As for computation time, RSM took approximately $50 \%$ more time than the RNG turbulence model which took a little bit more time than standard k- $\varepsilon$ turbulence model. Because of its unsteady nature, the LES model required 500\% more time than the RANS turbulence models. The contaminant concentration profiles shown at various locations in Figure 3.14 indicate that except very near the body all models exhibit similar results. However, as seen in Figure $3.12 \mathrm{~d}$ near the face of the body, LES results are significantly different than the other models. 


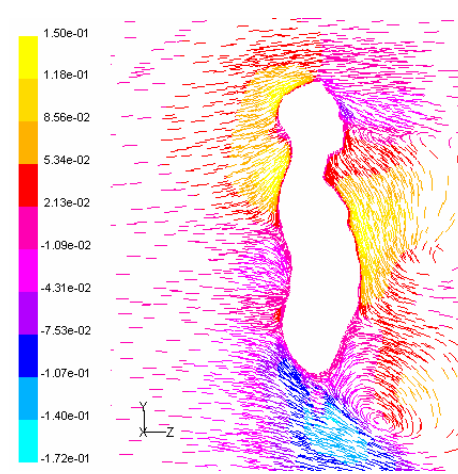

(a) Standard k- $\varepsilon$ turbulence model
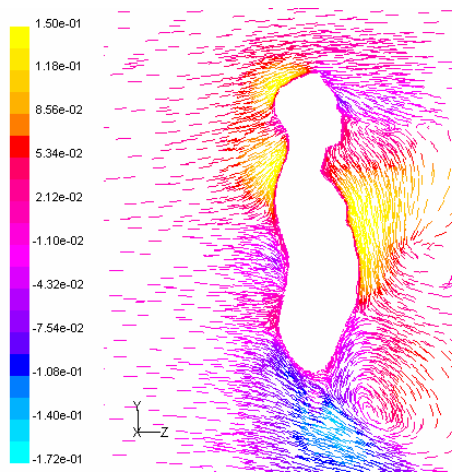

(c) RSM

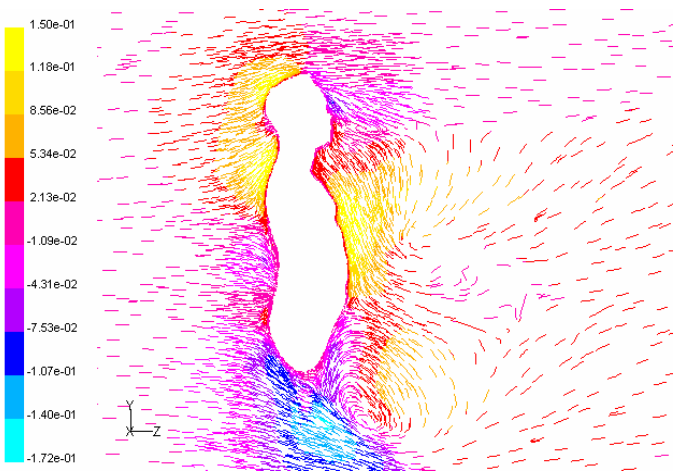

(b) RNG k- $\varepsilon$ turbulence model

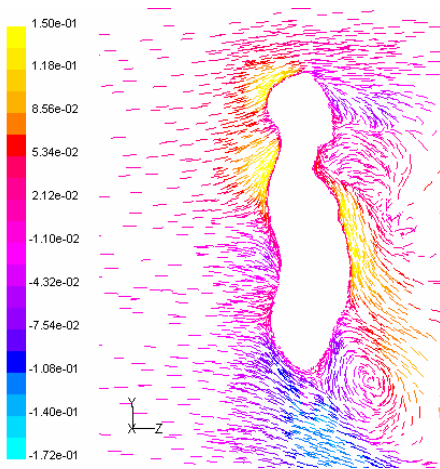

(d) LES (Averaged flow field)

Figure 3-12 Streamlines colored by y-velocity in the middle cross section plane with different turbulence models

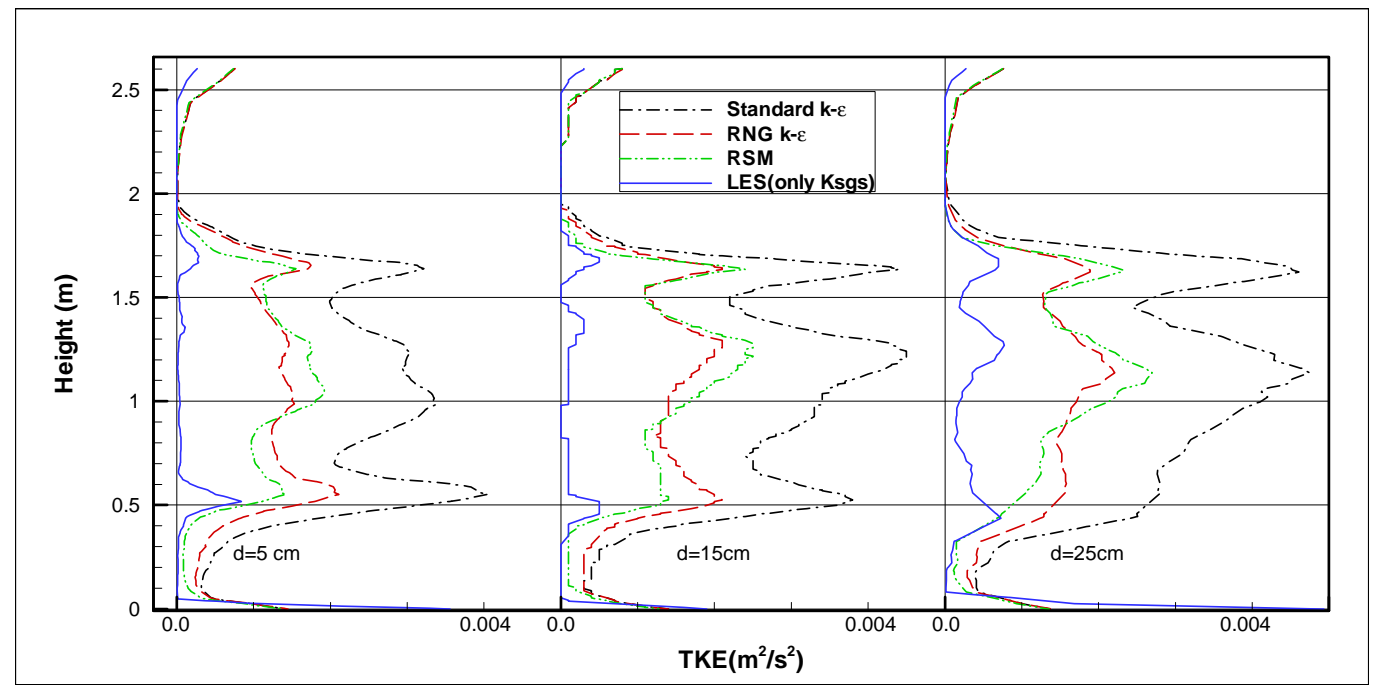

Figure 3-13 Tke/subgrid Tke at different horizontal distances from the mouth along a vertical line in the middle plane (Ksgs = Subgrid scale turbulent kinetic energy) 


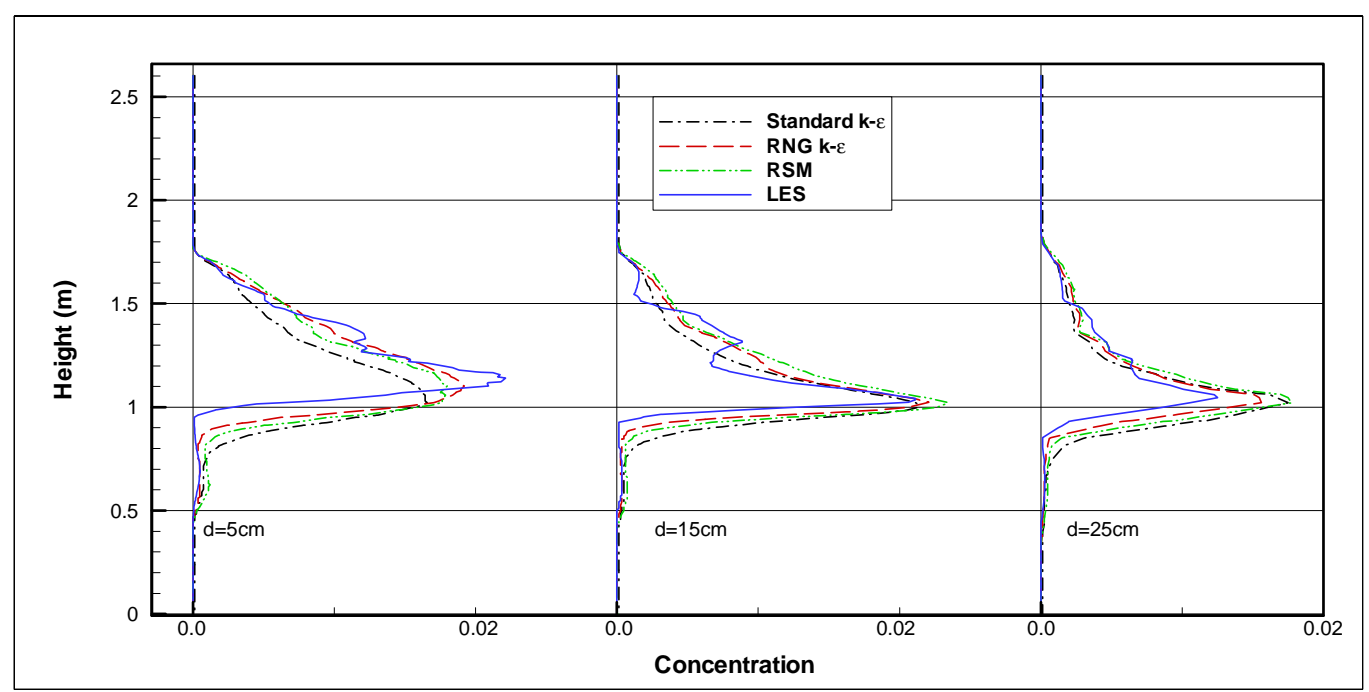

Figure 3-14 Scalar concentrations $\left(\mathrm{kg} / \mathrm{m}^{3}\right)$ at different horizontal distances from the mouth along a vertical line in the middle plane

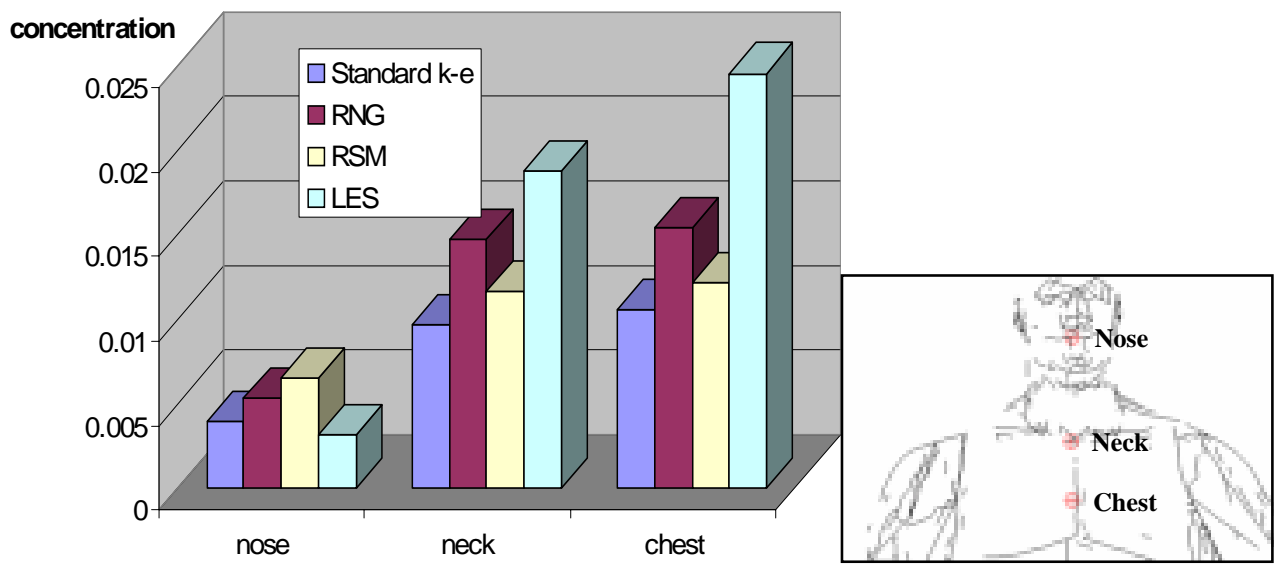

Figure 3-15 Concentration $\left(\mathrm{kg} / \mathrm{m}^{3}\right)$ levels at different sampling locations with different turbulence models; Eulerian scalar transport

The results from this study show that significant differences in predicted concentration levels are observed when different turbulence models are utilized. The coefficient of variation for species concentrations predicted with four different turbulence models ranged between $30-40 \%$. That is why one of the objectives of this study is to recommend a suitable turbulence model for worker exposure studies. 


\subsubsection{Effect of ventilation intensity, free stream turbulence, and heat flux from the body}

The simulations have been performed using the Fluent CFD software package (Fluent, Inc., Lebanon, NH). The geometrical configuration of the flow domain is shown in Figure 3.16, consisting of the wind tunnel $(4.6 \mathrm{~m}$ width $\times 2.6 \mathrm{~m}$ height $\times 7 \mathrm{~m}$ length), and a manikin $(1.69 \mathrm{~m}$ height $)$. The manikin body faces downstream of the isothermal flow. The fluid medium is air.

A source pan $(\phi=0.23 \mathrm{~m})$ as shown in Figure 3.17 is used to release the mixture of nitrogen and ethanol vapor from 90 small holes $(5 \mathrm{~mm})$ in the experimental study. The source pan is $1.04 \mathrm{~m}$ above the ground at the manikin's waist height and $0.25 \mathrm{~m}$ downstream of the manikin's torso. The mass flow rates of nitrogen and ethanol vapor are $1.98 \mathrm{e}-5 \mathrm{~kg} / \mathrm{s}$ and $9.34 \mathrm{e}-7 \mathrm{~kg} / \mathrm{s}$, respectively. The mass average velocity of the released mixture is $0.0026 \mathrm{~m} / \mathrm{s}$ in the vertical direction. The nitrogen is treated as air in the numerical study since this approximation greatly simplifies the calculation without loss of accuracy (By numerical experimentation it has been verified that the concentration at the breathing zone is not affected by the presence of the nitrogen). Source terms including mass, momentum in the vertical direction, as well as turbulence quantities such as $\mathrm{k}$ and $\varepsilon$ are added to the corresponding equations in the source pan region (the red region in Figure 3.16).

Constant velocity inlet and pressure outlet boundary conditions were used. The inlet velocity was varied in the range $0.051-0.762 \mathrm{~m} / \mathrm{sec}(10-150 \mathrm{fpm})$ with a turbulence intensity of $10 \%$, values representative of typical working environments (Baldwin and Maynard, 1998). The characteristic turbulent length scale was chosen to be $0.01 \mathrm{~m}$, which represents the turbulence generating honeycomb size at the inlet of the tunnel. At solid surfaces the usual non-slip and impermeability conditions are applied. The standard wall function is used for the calculation with turbulence models.

A rounded body, which is used in experiments and is thinner than the one used in the body shape study, is utilized in the simulations. Tetrahedral grids are generated. There are a total of $1,575,222$ tetrahedral cells. The smallest tetrahedral grid size on the manikin surface is around $0.005 \mathrm{~m}$. The mesh around the body is much thicker than in other regions. From the extensive grid study (Li, et al. 2005), it is expected that the grid convergence index (GCI) for the concentration at the breathing zone is less than 5\% since the grid used in this study is much finer than grid \#3 in the study for the body shape effect. 
The heat generation rate of the heated body in the experiments was approximately $90 \mathrm{~W}$. It is released from the upper-body surface (excluding the arms) by both convection and radiation. Whereas the heat released by the radiation was unknown, it is assumed that all the generated heat is released from the body by convection through the upper-body in the first run of the simulations with the heated body. This boundary condition is also called ' $\mathrm{B} 1$ ' in the later discussion. The second run is done after the experiments which provide temperature difference as listed in Table 3.3 between the upper-body and the inlet airflow. The averaged temperature is specified on the upper-body and the head for the thermal boundary condition in the second run. This is the 'B2' boundary condition referred to in the discussion that follows.

The Reynolds number (as listed in Table 3.3), which represents the ratio of the inertial force and the viscous force, is calculated by $\operatorname{Re}=U D / v$, where the $U$ is the inlet velocity and $\mathrm{D}$ is the equivalent dimensions of either the head or the shoulder. The Grashof number is about $8 \times 10^{8}$, which measures the ratio of the buoyancy force to the viscous force and is computed from $G r=\frac{g \beta \Delta T L^{3}}{v^{2}}$, where g is gravity, $\beta$ is the thermal expansion coefficient, $\Delta \mathrm{T}$ is the temperature difference, and $v$ is the kinematic viscosity.

The SIMPLE scheme was used to handle the pressure-velocity coupling. QUICK Scheme was applied for the convection terms in the momentum and scalar transport equations. The second order upwinding scheme was used for the convection terms in the $\mathrm{k}$ and $\varepsilon$ equations. Central differencing was utilized for the diffusion terms in all equations.

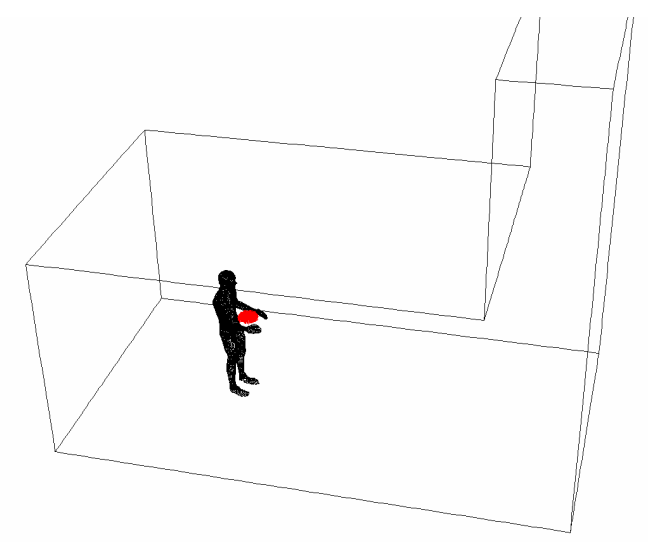

Figure 3-16 Schematic view of the computational domain (short domain) 


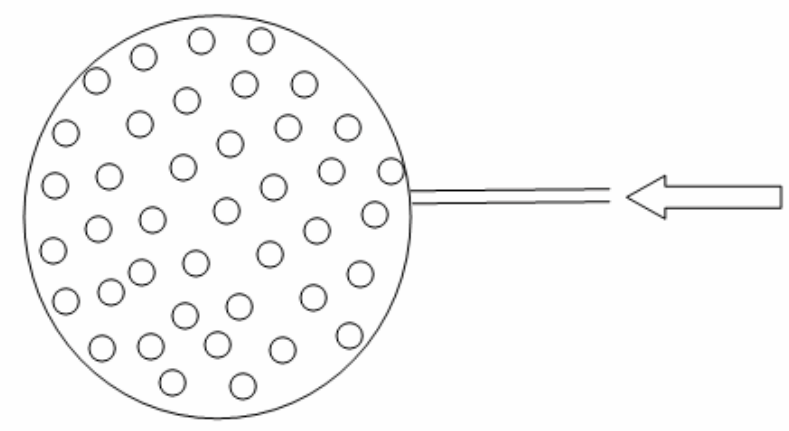

Figure 3-17 Top view of the source pan

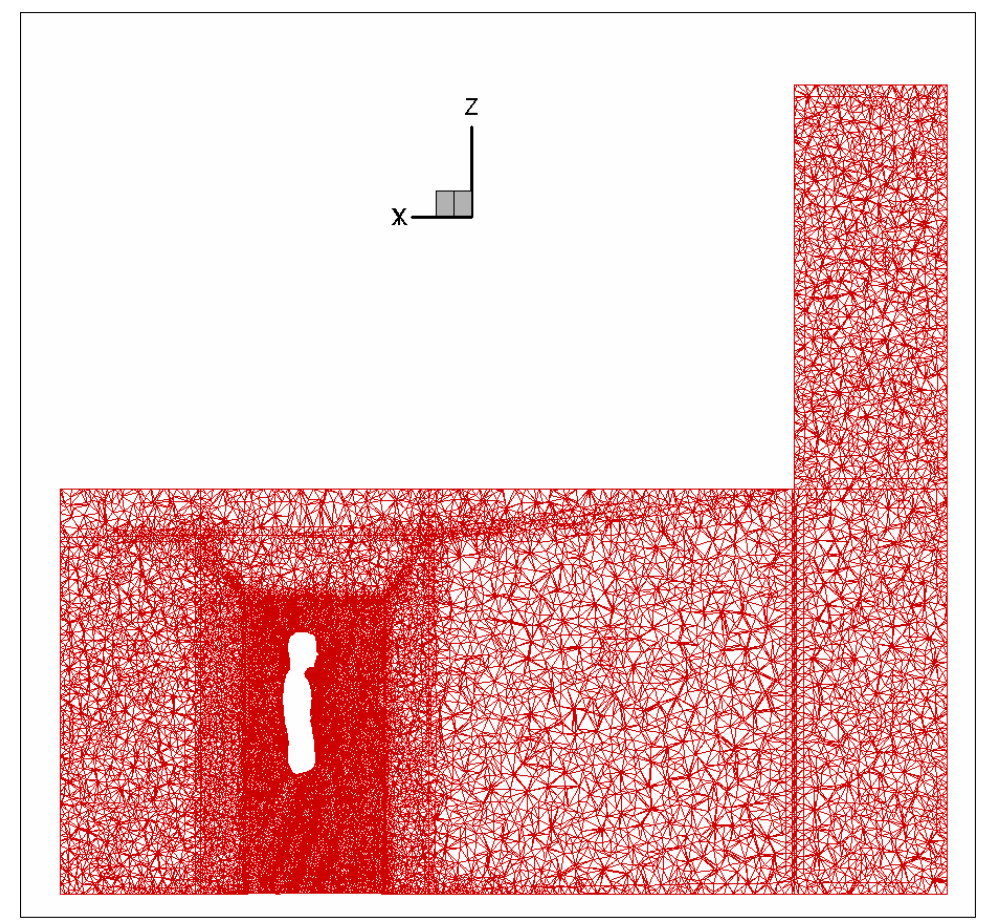

Figure 3-18 The computational mesh in the center plane 
Table 3.3 Reynolds number at different ventilation intensity

\begin{tabular}{|l|r|r|r|r|r|r|r|}
\hline$U$ (inlet) (fpm) & 10 & 20 & 40 & 60 & 80 & 100 & 120 \\
\hline$U$ (inlet) $(\mathrm{m} / \mathrm{s})$ & 0.05 & 0.10 & 0.20 & 0.30 & 0.41 & 0.51 & 0.61 \\
\hline $\operatorname{Re}($ based on head size) & 686 & 1373 & 2746 & 4119 & 5492 & 6865 & 8238 \\
\hline $\operatorname{Re}$ (based on shoulder size) & 2073 & 4147 & 8294 & 12441 & 16588 & 20735 & 24882 \\
\hline Averaged temperature difference(K) & 9 & 9 & 10 & 10 & 10 & 9 & 9 \\
\hline
\end{tabular}

\section{Sensitivity of RNG turbulence model to the low Reynolds number modification}

Before the effect of Re is analyzed, some issues should be addressed on the unsteadiness of the flow. It can be seen from Figure 3.19 that the concentration at the breathing zone (represented by a point 1 cm downstream of the mouth) predicted with low_Re RNG varies significantly with iteration (pseudo time marching). Without appropriate averaging, the instantaneous concentrations calculated with low_Re RNG should not be compared with the experimental data, since the measurements in the experiments were obtained on a relatively long time (15 minutes) sampling, and hence represent ensemble averaged values. To obtain reasonable mean concentrations, at least 1000 iterations are necessary for low_Re RNG, but 500 iterations would be enough for RNG model. Simulations with low_Re RNG demand more computational cost than that with RNG. The mean data shown in Figure 3.20 are averaged values of 1000 iterations for low_Re RNG and laminar flow, and 500 iterations for RNG. A detailed LES simulation could provide the typical frequencies of the flow, which could clarify if 15 -minute sampling time is sufficient for the contaminant sampling.

Figure 3.20 presents the comparison of the numerical and experimental results of the concentration at the breathing zone with RNG, low-Re RNG and laminar flow for the unheated case. The numerical results with both low_Re RNG and RNG agree well with each other. At low Reynolds number (V(inlet)< $30 \mathrm{fpm}(0.15 \mathrm{~m} / \mathrm{s}))$, low_Re RNG and RNG result in $20-30 \%$ difference for the exposure levels in the breathing zone. The results predicted with RNG turbulence model are closer to experimental data, although the calculations with low-Reynolds number modification also agree satisfactorily with the experimental results. The concentrations in the breathing zone simulated with the laminar flow are the largest among the three choices. Since the RNG turbulence model requires less computational effort and achieves relatively 
better agreement with experiment than the low-Re version, it is chosen to serve as the turbulence model for the subsequent evaluation of the effect of the free stream turbulence and the heat release from the body.

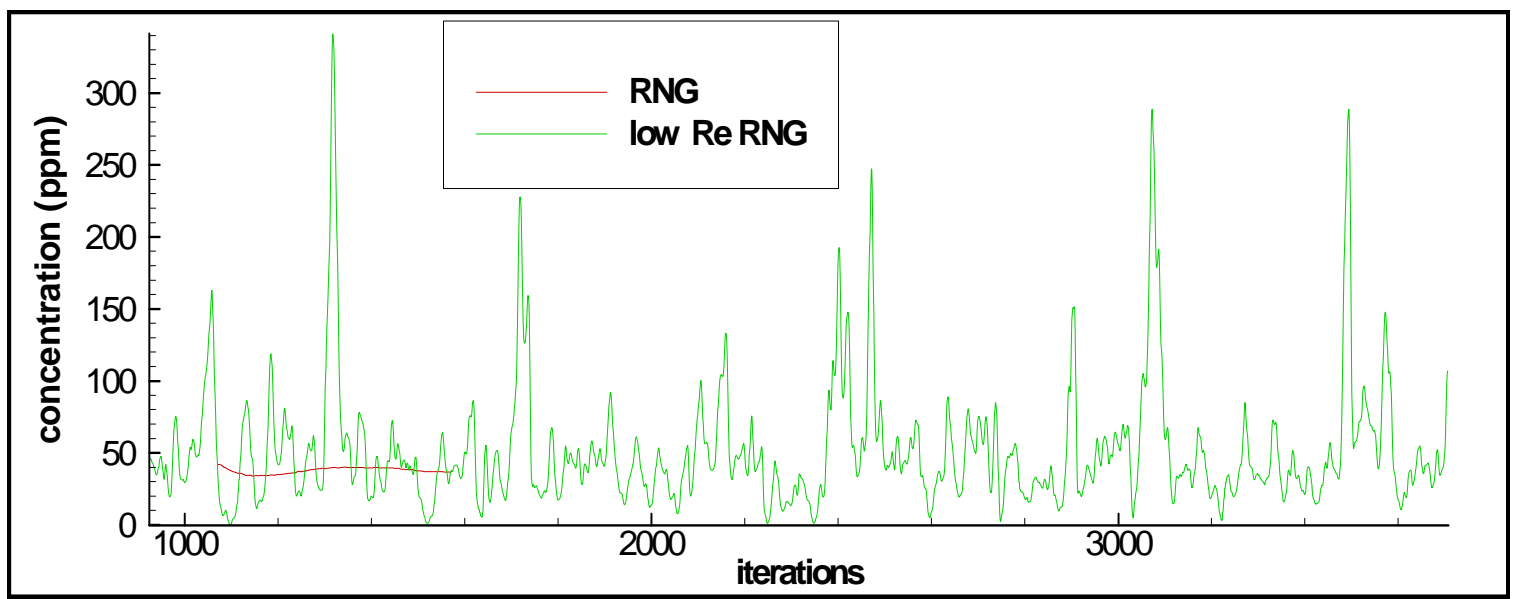

Figure 3-19 Concentration at the breathing zone vs. iterations at an inlet velocity of $30 \mathrm{fpm}(0.15$ $\mathrm{m} / \mathrm{s})$

Both experiments and simulations (Figure 3.20) show that, with the unheated body, the concentrations at the breathing zone decrease as the ventilation intensifies, which agrees with the experimental results by George et al (1990). The exposure levels decline quickly especially when the inlet velocity is less than $50 \mathrm{fpm}(0.25 \mathrm{~m} / \mathrm{s})$. The concentrations at the breathing zone change less in the $\mathrm{Re}$ range of $50-150 \mathrm{fpm}(0.25-0.75 \mathrm{~m} / \mathrm{s})$. Overall, the predictions with the RNG turbulence model agree very well with the experimental data. The only exception is in the low Reynolds number region, e.g., $V($ inlet $)=10 \mathrm{fpm}$, where the simulated concentration is much higher than the measurements.

The effect of the free stream turbulence on the worker exposure is presented in Figure 3.21. It is seen that the overall trend for the concentration at the breathing zone goes down as the free stream turbulence intensifies, despite of the marginal change as turbulence intensity varies from $10 \%$ to $40 \%$. At $\mathrm{V}($ inlet $)=10 \mathrm{fpm}(0.05 \mathrm{~m} / \mathrm{s})$, the exposure level descends from $107 \mathrm{ppm}$ to $55 \mathrm{ppm}$ as the turbulence intensity increase from $40 \%$ to $60 \%$. In the experiments, higher turbulence intensity has been observed at the low Reynolds number (Welling, et al. 2000; Guffey, 2004), which may be the reason for the discrepancy between the simulations and the experiments shown in Figure 3.20. 


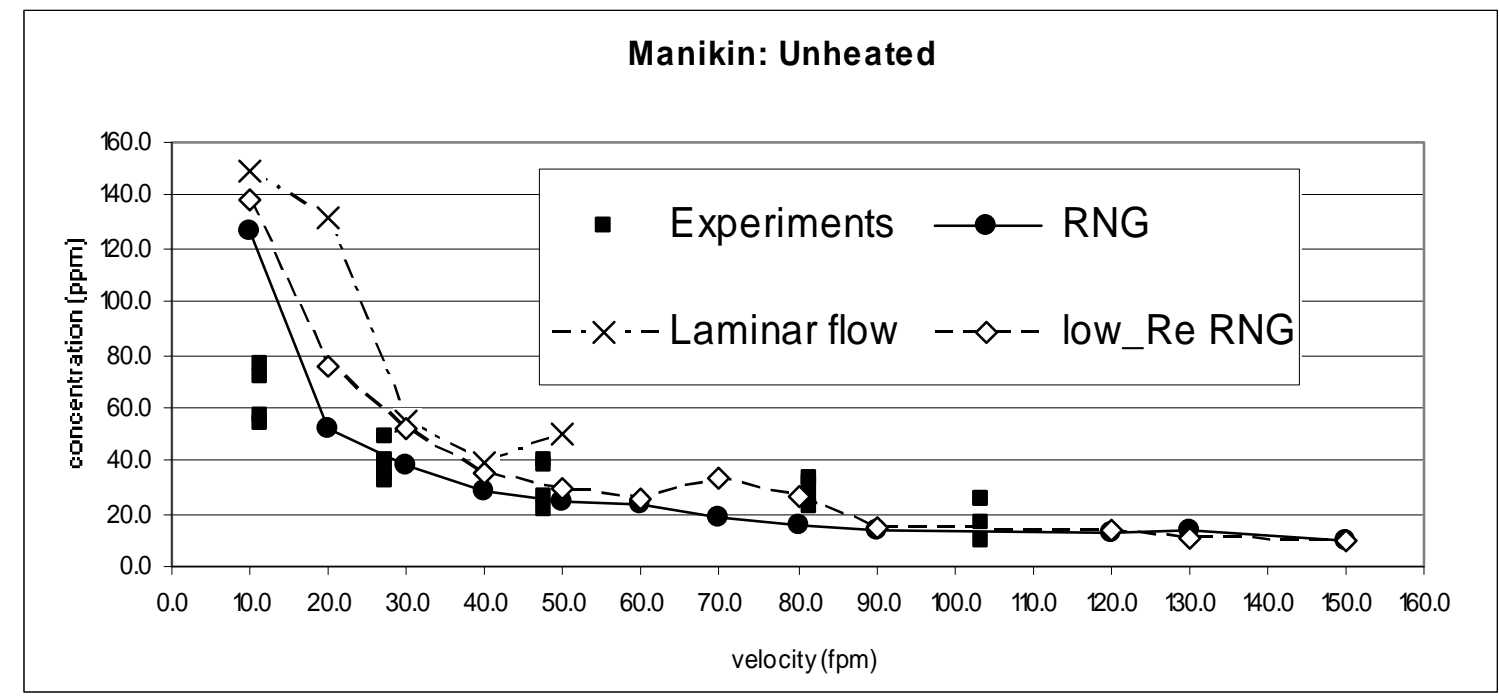

Figure 3-20 Comparison of the numerical and experimental results of the concentration at the breathing zone; free stream turbulence level is $10 \%$.

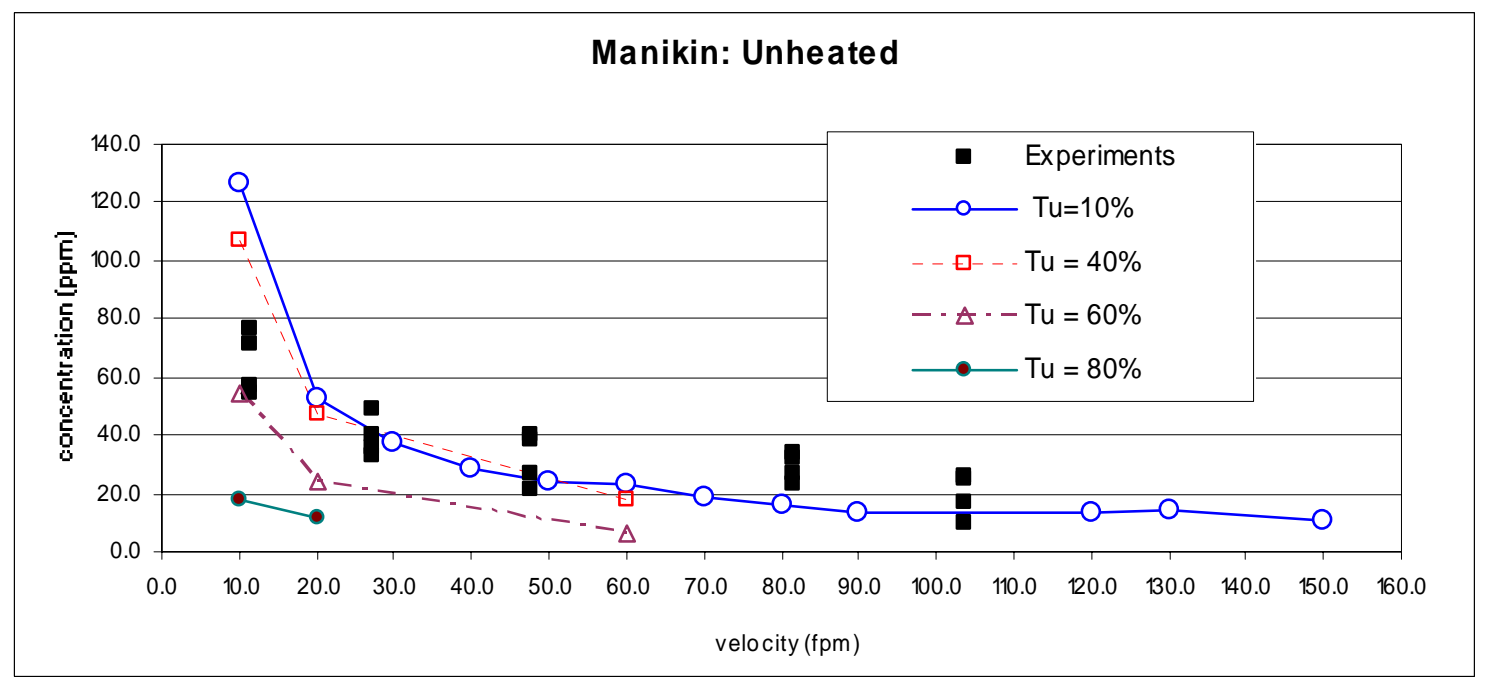

Figure 3-21 The effects of the ventilation intensity and the free stream turbulence on the concentration at the breathing zone (with RNG turbulence model)

Nevertheless, with the heated body, the trend was very different, as is shown in Figure 3.22. The concentration at the breathing zone first increases as the ventilation intensity increases. The exposure levels reach a peak at a ventilation velocity of around 40-60 fpm. Then it drops as the velocity increases further. 
Again, the predictions with the temperature boundary conditions agree well with the measurements. The calculations with full heat convection (B1) results in lower predicted exposure levels than the one measured in the experiments. This suggests that the worker would benefit from less radiation if the heat he generates remains constant. However, it is not convenient to control the radiation in practice. Furthermore, the heat flux by sweating/evaporative cooling is not considered in the current experiments and simulations. The evaporative cooling is not negligible when the worker is at a heavy duty. More importantly it affects the comfort level of the worker. Comprehensive consideration on both the exposure and the comfort level index would be desired for today's working environment.

It is interesting to note that the exposure levels from numerical simulations for the heated body show a zigzag (i.e. a wavy) pattern from $V($ inlet $)=30 \mathrm{fpm}(0.15 \mathrm{~m} / \mathrm{s})$ to $V($ inlet $)=50 \mathrm{fpm}(0.25 \mathrm{~m} / \mathrm{s})$ when the temperature boundary condition (B2) is specified. Although the experimental data is too coarse in this region to verify this, the relatively larger variation at $\mathrm{V}($ inlet $)=47 \mathrm{fpm}(0.24 \mathrm{~m} / \mathrm{s})$ may indicate that the slope of the curve should be larger than the one formed by connecting the experimental data by a straight line. The reason for the zigzag pattern could be due to the alternating dominance of the buoyancy force and the inertial force. Even wider zigzag region is found in the simulations with heat flux specified (B1).

Comparing Figure 3.20 and Figure 3.22, it can be stated that the exposure levels with the heated body are higher than the ones with the unheated body in the V(inlet) range of 30-100 fpm. Nevertheless, at low Reynolds number $(\mathrm{V}(\mathrm{inlet})=10)$, the exposure levels with the heated body are lower. Keeping this in mind and more discussion will be provided later when detailed flow field information is presented.

The convection and the radiation heat release rates are plotted against the ventilation intensity in Figure 3.23. The convection heat release is computed by integrating the heat convection on the whole upper-body, and the radiation is calculated by subtracting the convection from the total heat generation rate. It is seen that, as the ventilation intensifies, the heat release via convection is also intensified. It is interesting that a rough calculation yields $59 \mathrm{~W}$ for the radiation heat transfer, based on an emissivity of 0.96 for the manikin (Bräuer et al. 2002). This further confirms the current simulations. 


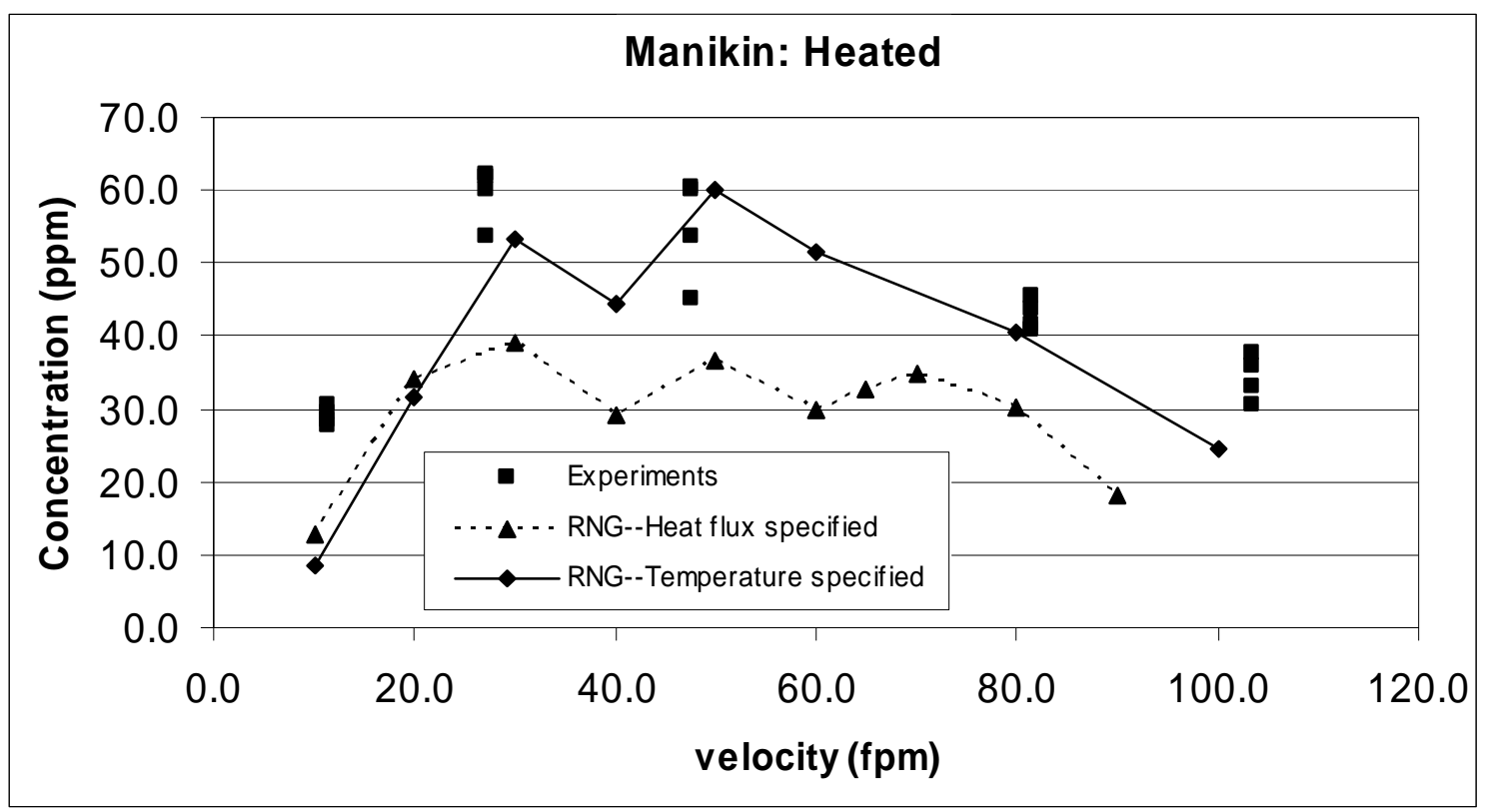

Figure 3-22 The effect of the ventilation intensity and the body heat on the concentration at the breathing zone; $\mathrm{Tu}=10 \%$

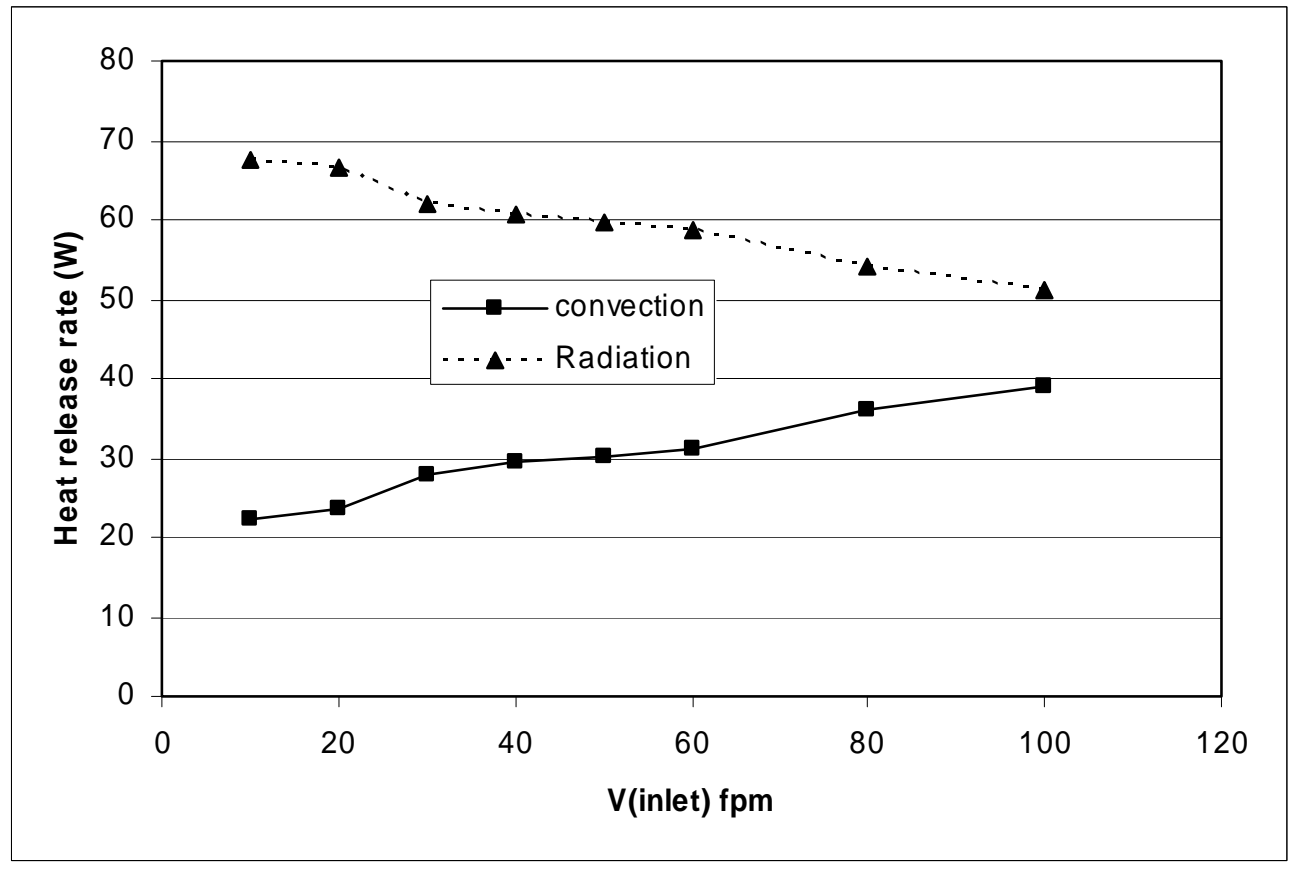

Figure 3-23 Heat flux via convection and radiation with RNG turbulence model and averaged temperature boundary condition (B2) 
The concentrations at the chest level are shown in Figure 3.24 and Figure 3.25. The computations capture the same trend as the experiments. By contrast, there is a $70-80 \%$ difference between the simulation results and the experimental data for the heated body in the $\mathrm{V}$ (inlet) range of 10-50 fpm. It seems that the free stream turbulence intensity influences the concentration with the unheated body, but it remains to be clarified whether the turbulence intensity has the same effect for the cases with the heated body.

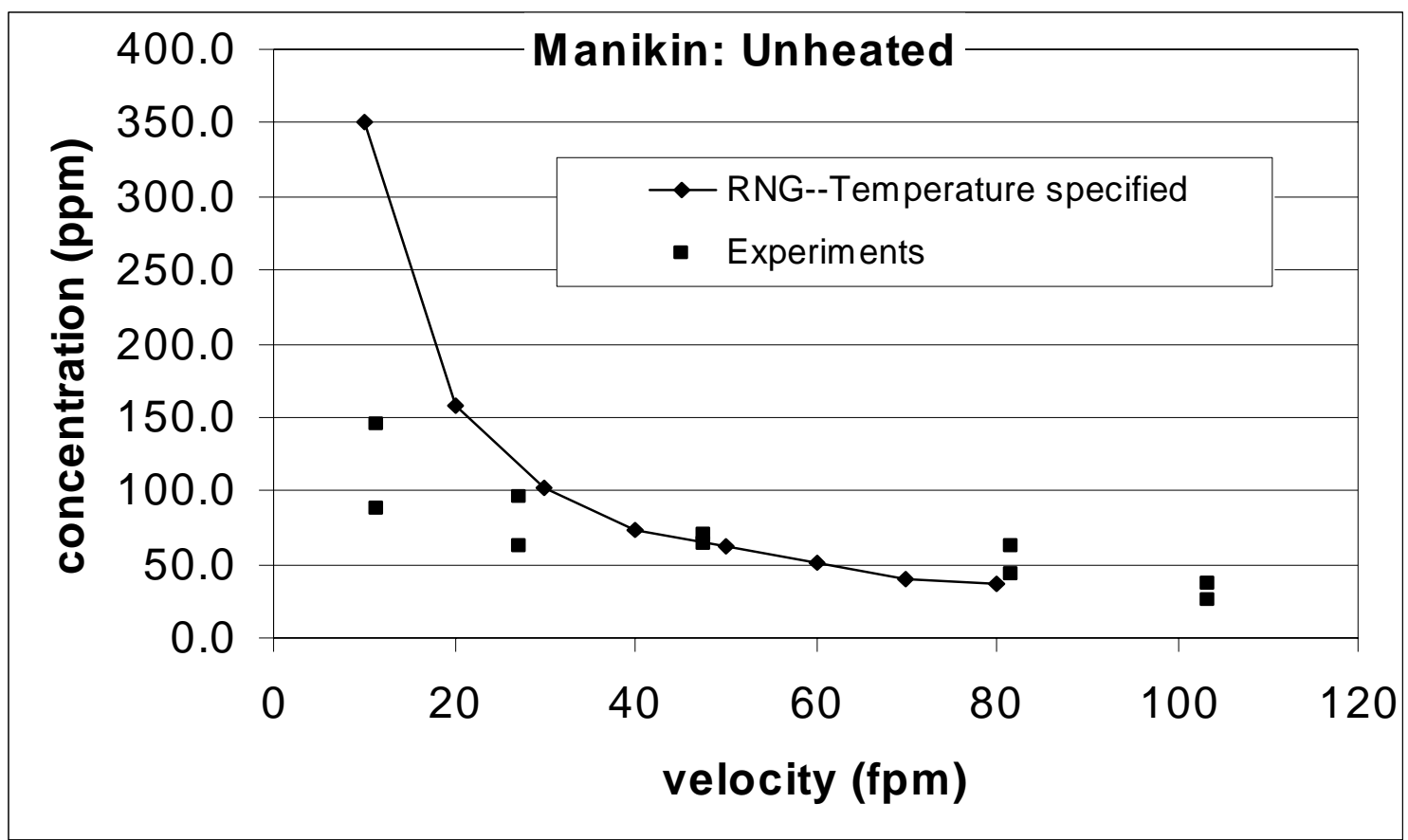

Figure 3-24 The effect of the ventilation intensity on the concentration at the chest 


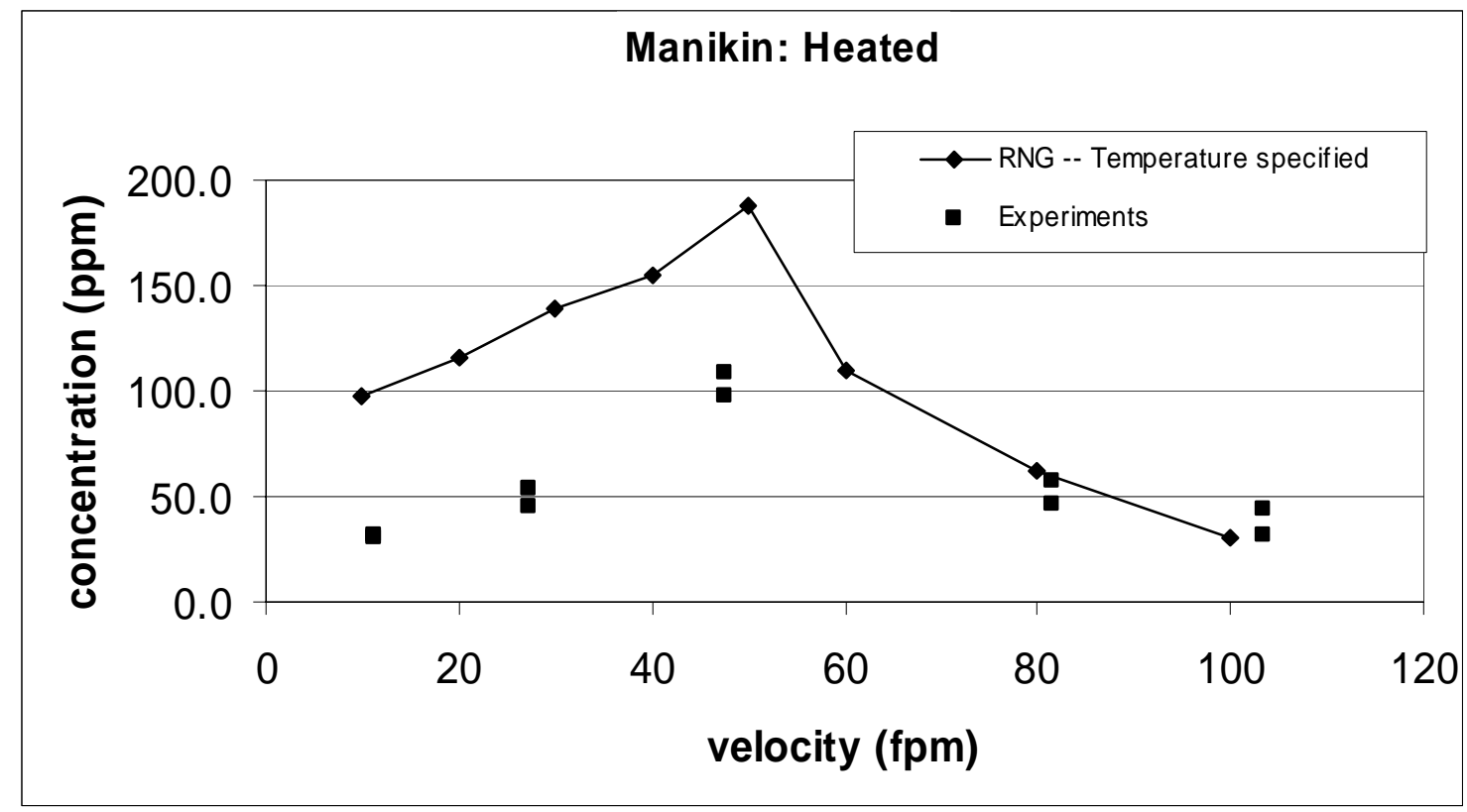

Figure 3-25 The effect of the ventilation intensity and the body heat on the concentration at the chest level

The turbulent kinetic energy contours and pathlines in the middle plane at different Reynolds number are presented in Figure 3.26. It is shown in Figure 3.26(a) that there are two recirculation zones in the immediate downstream of the unheated body at $\mathrm{V}$ (inlet) $=10 \mathrm{fpm}$, whereas, with the heated body, there is no recirculation region found in Figure 3.26(b). The heat flux from the body significantly affects the flow field and the turbulent kinetic energy in the downstream of the body. With the heated body, the characteristic buoyant velocity is around $0.2 \mathrm{~m} / \mathrm{s}$. At $\mathrm{V}($ inlet $)=10 \mathrm{fpm}(0.05 \mathrm{~m} / \mathrm{s})$, the upwards convection induced by the buoyancy is dominant so that there is no recirculation region formed at the breathing zone. The heat flux also increases the turbulence level around the body. All these effects consequently result in significantly different concentration distributions (as shown in Figure 3.27(a) and (b)). The exposure level in the breathing zone with heated body is much lower than the one with unheated body.

As the inlet velocity increases to $60 \mathrm{fpm}(0.3 \mathrm{~m} / \mathrm{s})$ (Figure $3.26(\mathrm{c}))$, the flow structure predicted with the unheated body is similar as the pattern at $V($ inlet $)=10 \mathrm{fpm}$, whereas, the turbulent kinetic energy in the recirculation regions increases significantly, which leads to more diffusion, hence lower concentration in the breathing zone than at $\mathrm{V}($ inlet $)=10 \mathrm{fpm}$ (Figure 3.27(a)). The natural convection 
induced by the body heat at $\mathrm{V}($ inlet $)=60 \mathrm{fpm}$ (Figure 3.26(d)) brings the contaminant directly to the breathing zone, and at approximately the forehead height; then it is stopped by the ventilation airflow and changes its direction. This procedure retards the convection of the ethanol vapor out of the breathing zone, which results in a much higher exposure level than the one at $\mathrm{V}($ inlet $)=10 \mathrm{fpm}$ (Figure 3.27(d) and 3.27(b)).

Note that the only difference between Figure 3.26(d) and (e) is the thermal boundary condition. With full convection (Figure 3.26(e)), the turbulent kinetic energy is larger than the one with partial convection (Figure 3.26(d)), which explains that the exposure level with full convection at $V($ inlet $)=60 \mathrm{fpm}$ is greater the one with partial convection at the same ventilation intensity (comparing Figure $3.27(\mathrm{~d})$ and $3.27(\mathrm{e}))$.

As the ventilation intensifies further, i.e., $\mathrm{V}($ inlet $)=100 \mathrm{fpm}$, the ventilation convection becomes dominant, and it induces a big recirculation region, which confines the ethanol vapor in the lower body region as shown in Figure 3.26(f).

It should be noted that the heat is released from the upper-body surface in this study to match the experimental condition. If the heat is released from the whole body, the Grashof number is expected to increase mainly due to the increasing of the length scale, hence, the peak in Figure 3.22 will move toward higher ventilation intensity. To the opposite side, if the heat is released from only the head (supposing that the rest of the body is covered by insulated work clothing), the Grashof number will decrease and the peak will move toward a lower ventilation intensity.

It should also be noted that the B2 boundary condition was imposed by the assumption of a uniform temperature distribution on the body. In the experiments with a heated manikin without clothing, the temperature measured on the head is $2^{\circ} \mathrm{C}$ different from the one on the abdomen. The temperature distribution on a real human body could be significantly non-uniform, due to clothing. This may contribute to the uncertainty of the predictions and may be considered in further study. 


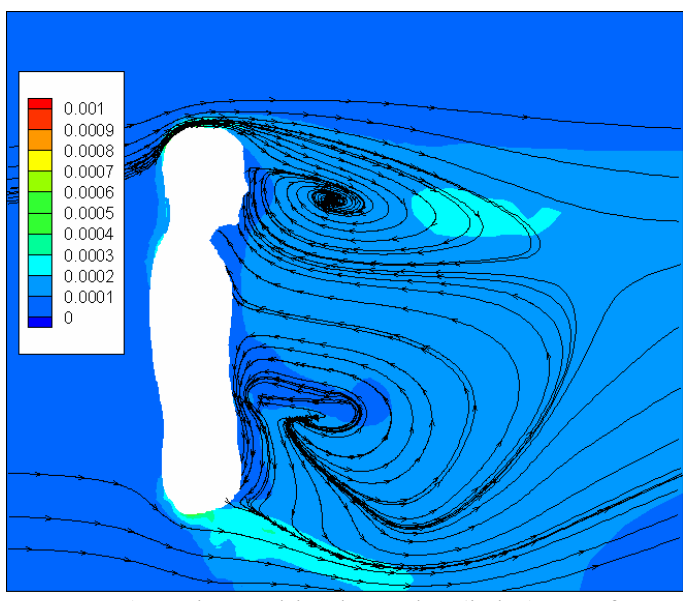

a) Unheated body and $V($ inlet $)=10 \mathrm{fpm}$

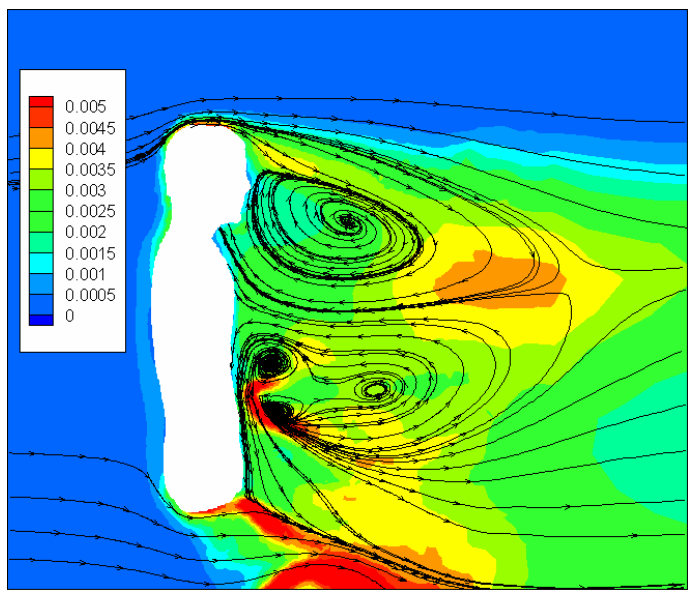

c) Unheated body and V(inlet) $=60 \mathrm{fpm}$

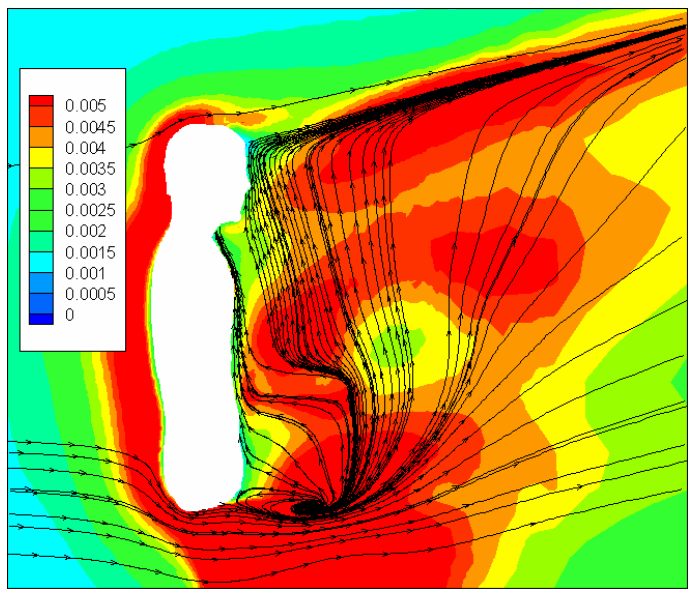

e) Heated body and V(inlet) $=60$ fpm (B1)

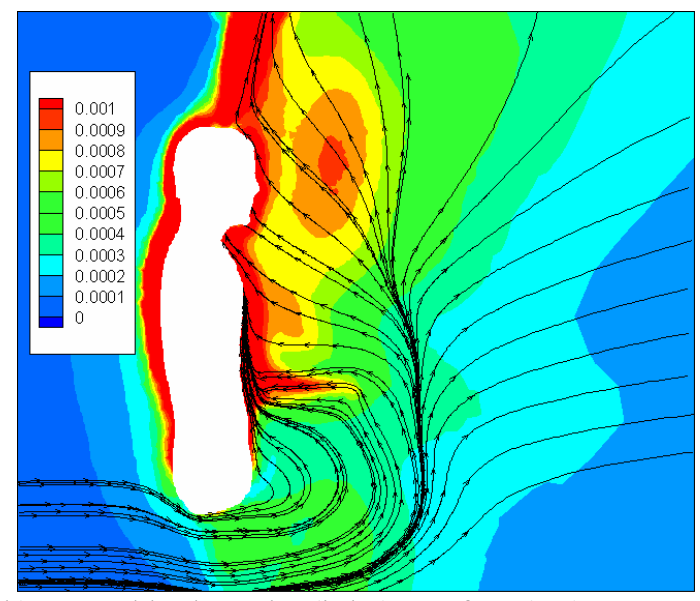

b) Heated body and V(inlet) $=10 \mathrm{fpm}$ (B2)

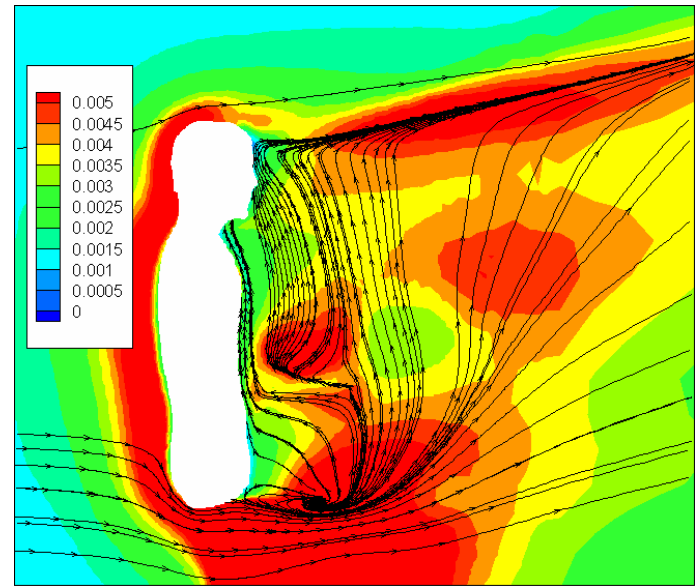

d) Heated body and V(inlet) $=60 \mathrm{fpm}(\mathrm{B} 2)$

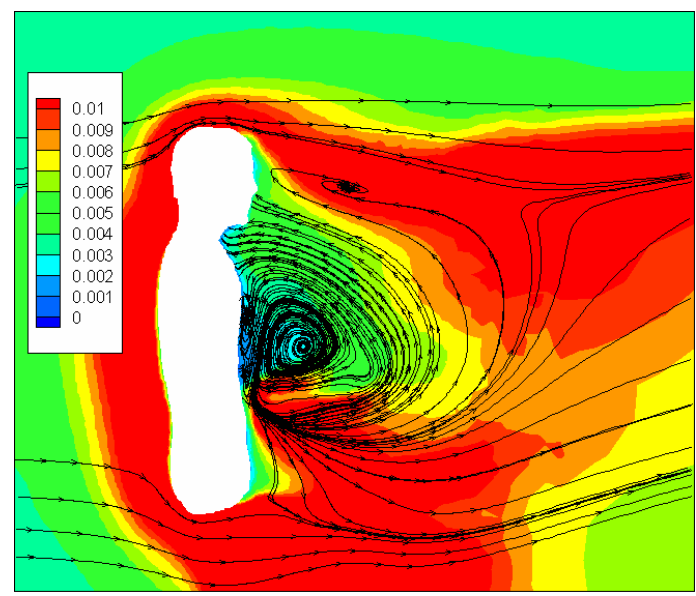

f) Heated body and V(inlet)=100 fpm(B2)

Figure 3-26 Turbulent kinetic energy contours and pathlines in the middle plane at different ventilation intensity with unheated and heated bodies 


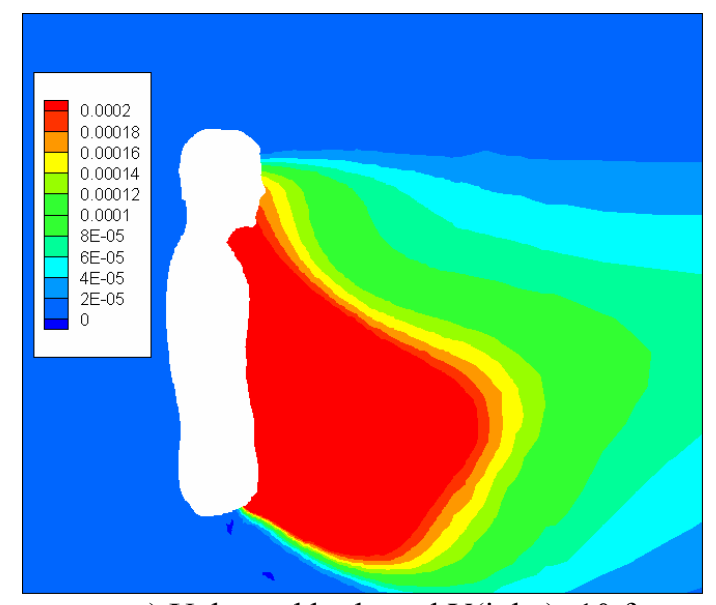

a) Unheated body and V(inlet) $=10 \mathrm{fpm}$

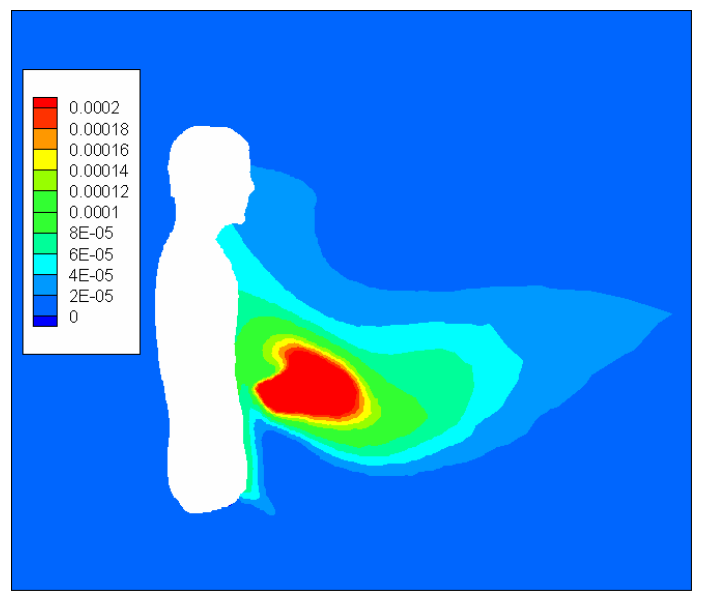

c) Unheated body and V(inlet) $=60 \mathrm{fpm}$

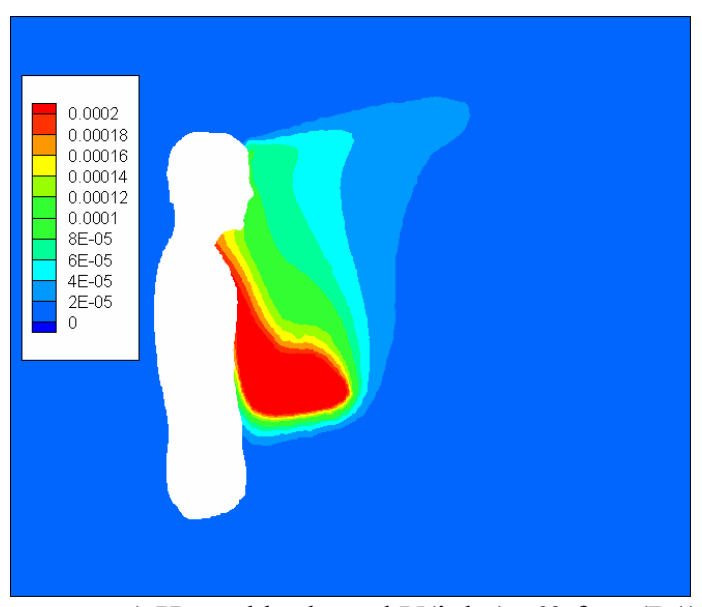

e) Heated body and V(inlet) $=60 \mathrm{fpm}(\mathrm{B} 1)$

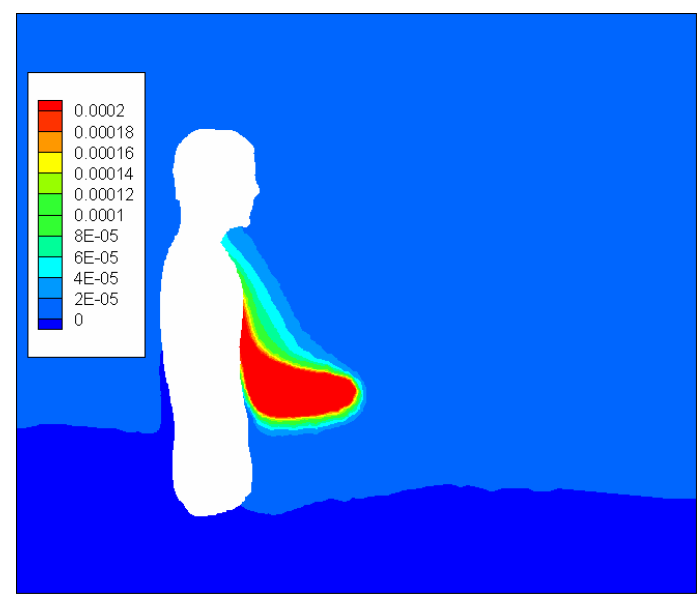

b) Heated body and V(inlet)=10 fpm (B2)

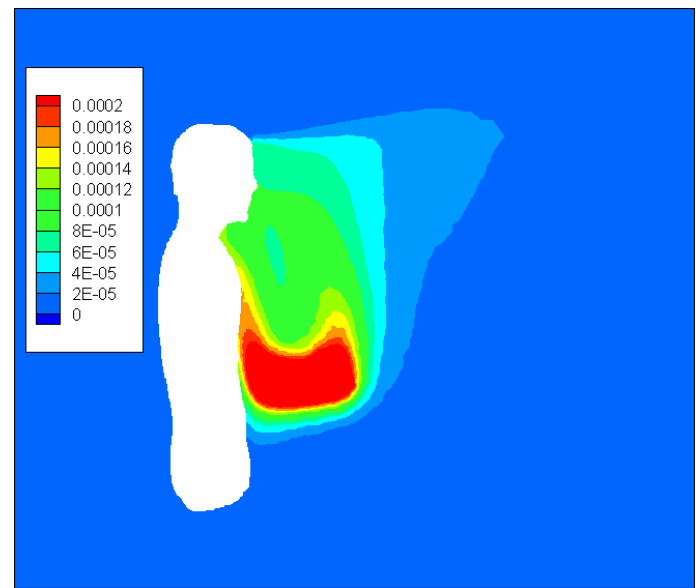

d) Heated body and V(inlet) $=60 \mathrm{fpm}(\mathrm{B} 2)$

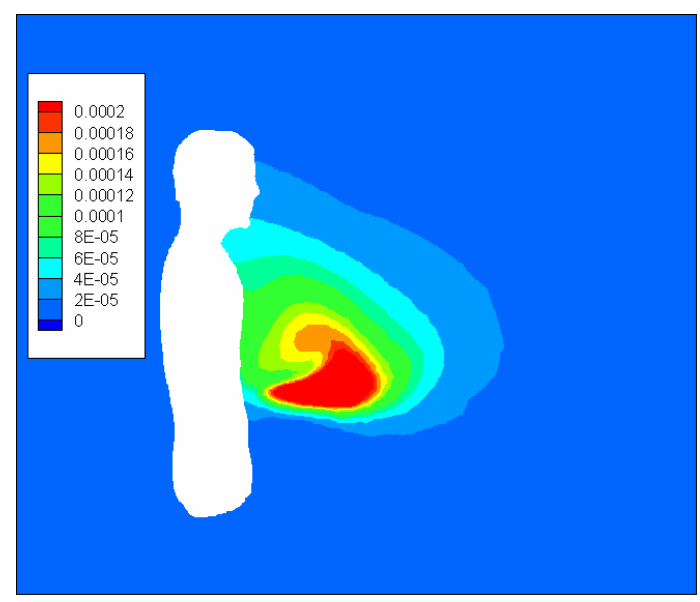

f) Heated body and V(inlet)=100 fpm(B2)

Figure 3-27 Concentration contours and pathlines in the middle plane at different ventilation intensity with unheated and heated bodies 
Figure 3.28 shows the effect of the free stream turbulence on flow around the unheated body. A turbulent intensity of $60 \%$ is used at inlet for this study. Comparing Figure 3.28(a) with Figure 3.26(a), larger turbulent kinetic energy is observed in the recirculation regions with $\mathrm{Tu}=60 \%$, which results in a more smeared concentration field as shown in Figure 3.28(b) than the one with $\mathrm{Tu}=10 \%$ (Figure 3.27(a)).

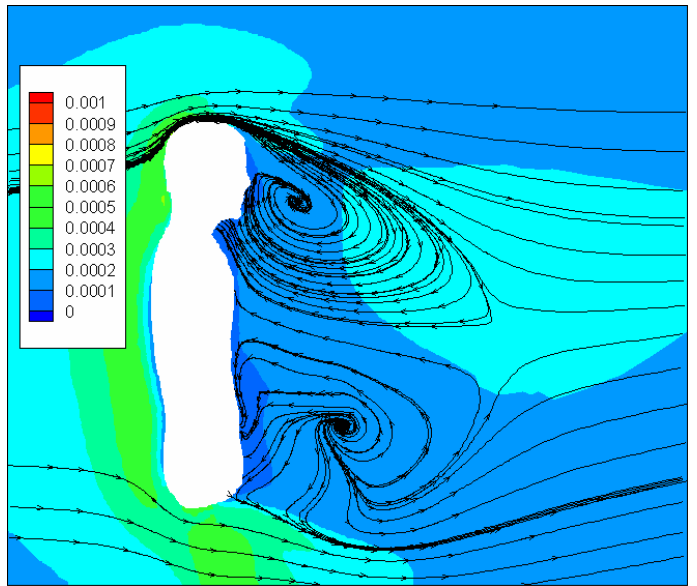

a) $\mathrm{k}$ contours and pathlines

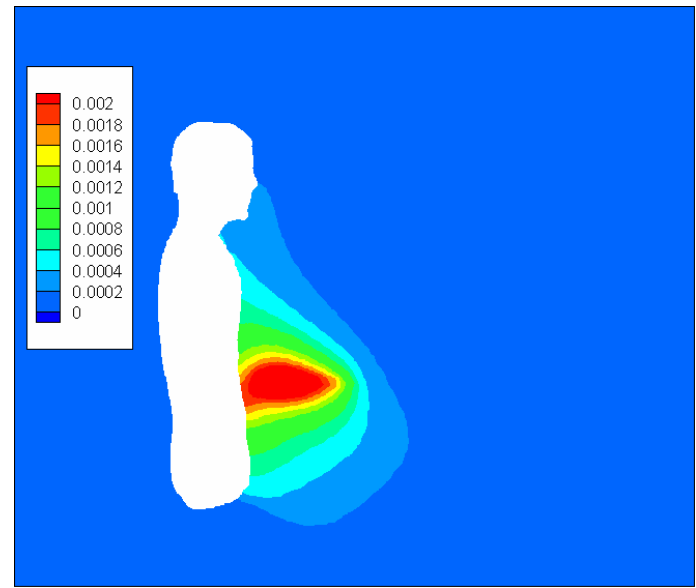

b) Concentration contours

Figure 3-28 The effect of the free stream turbulence with the unheated body at $V$ (inlet) $=10 \mathrm{fpm}$ and $\mathrm{Tu}=60 \%$

\subsubsection{Flow unsteadiness}

A closer examination of the flow unsteadiness study was aimed to understand the flow dynamics in the formation and the development of the wake flow in the downstream of human body as well as the relation to the contaminant transport. A LES run was conducted since the LES approach is conceptually more suitable in such situations as it resolves the large-scale unsteady motions and requires modeling of only the small-scale turbulent motion which is less influenced by the boundary conditions.

The same grid as shown in Figure 3.18 is utilized. The inlet velocity for this case is $20 \mathrm{fpm}$. A time step of $0.5 \mathrm{~s}$ was used, which is about 10 times larger than the Kolmogorov time scale and is sufficient for the LES simulation in this case according to Frisch (1995). The Smagorinsky subgrid scale model is used for the prediction of small scale turbulence. A zero initial velocity field is specified at $t=0 \mathrm{~s}$. The inlet turbulence is assumed isotropic and with an intensity of $10 \%$. Simulation results in the first two flow- 
through times are discarded to allow the flow formation and developing. The maximum $y^{+}$of the first cells on the body is around 4 .

Instantaneous pathlines and the concentration contours in the middle plane at $\mathrm{t}=600 \mathrm{~s}$ are shown in Figure 3.29. It is seen that flow separates on the forehead and forms an eddy in the downstream of the head, and another eddy is developed in the chest region. It seems that not much ethanol is transported from the lower eddy to the upper one. At the same moment, if observed from a horizontal plane $(z=1.51 \mathrm{~m})$ cutting through the breathing zone (Figure 3.30), the instantaneous flow seems to be asymmetric and highly three-dimensional. Figure 3.31 shows the flow on a horizontal plane across the waist $(\mathrm{z}=1.16 \mathrm{~m})$. Again, the flow is asymmetric and three-dimensional. Eddies are not only formed at the shear layer along the arms, but triggered by the leaking jet flow between the arms and the body. Considering the interaction of the jet flow and the wake, the flow pattern in this case is certainly more complicated than simple flows such as the flow over a circular cylinder. The flow structure in the middle plane at $t=620 \mathrm{~s}$ (Figure 3.32) is totally different from the one at $20 \mathrm{~s}$ earlier (Figure 3.29).

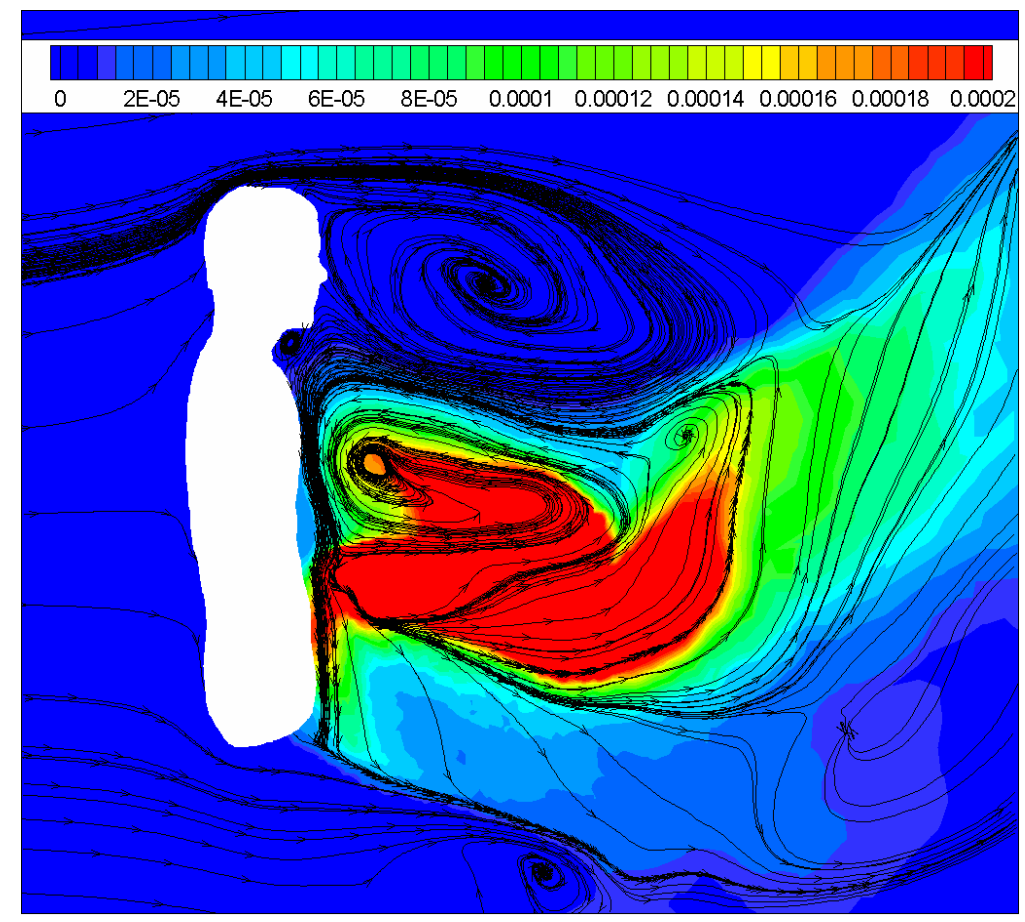

Figure 3-29 Instantaneous Pathlines and concentration contours in the middle plane at $t=600 \mathrm{~s}$ (concentration :kg/kgair) 


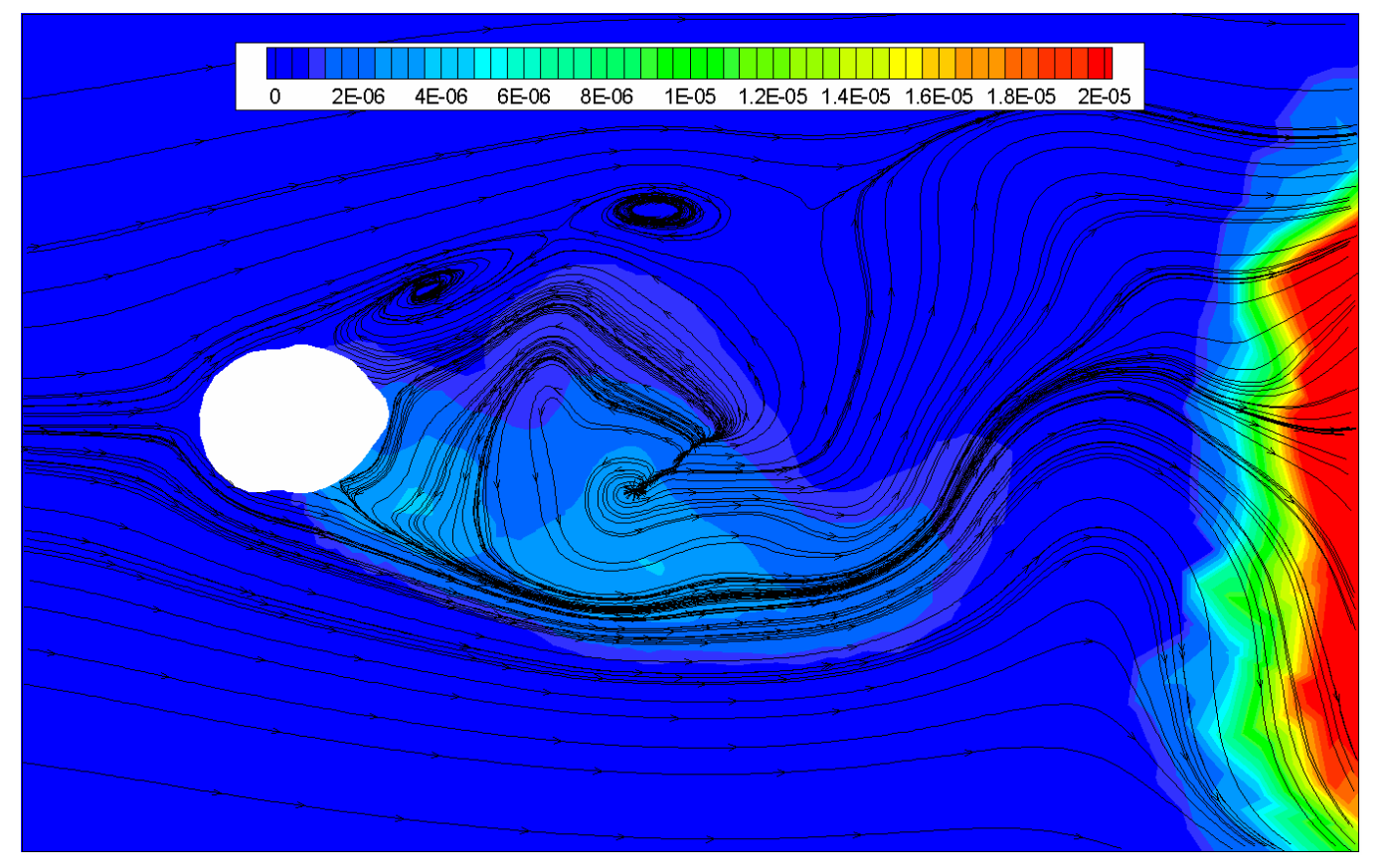

Figure 3-30 Instantaneous Pathlines and concentration contours in the horizontal plane $z=1.51$ $\mathrm{m}$ at $\mathrm{t}=600 \mathrm{~s}$ (concentration $: \mathrm{kg} / \mathrm{kgair}$ )

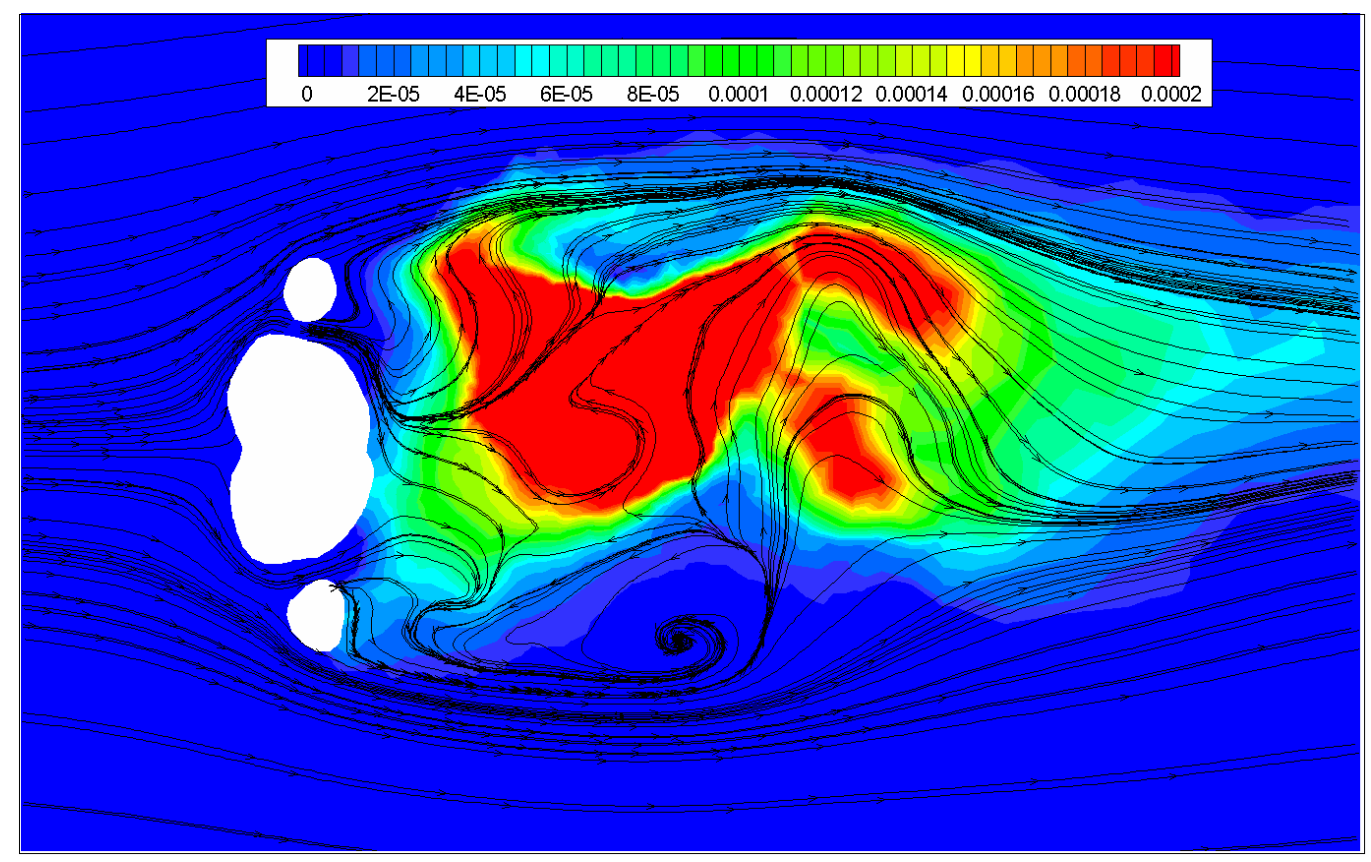

Figure 3-31 Instantaneous Pathlines and concentration contours in the horizontal plane $z=1.16$ $\mathrm{m}$ at $\mathrm{t}=600 \mathrm{~s}$ (concentration :kg/kgair) 


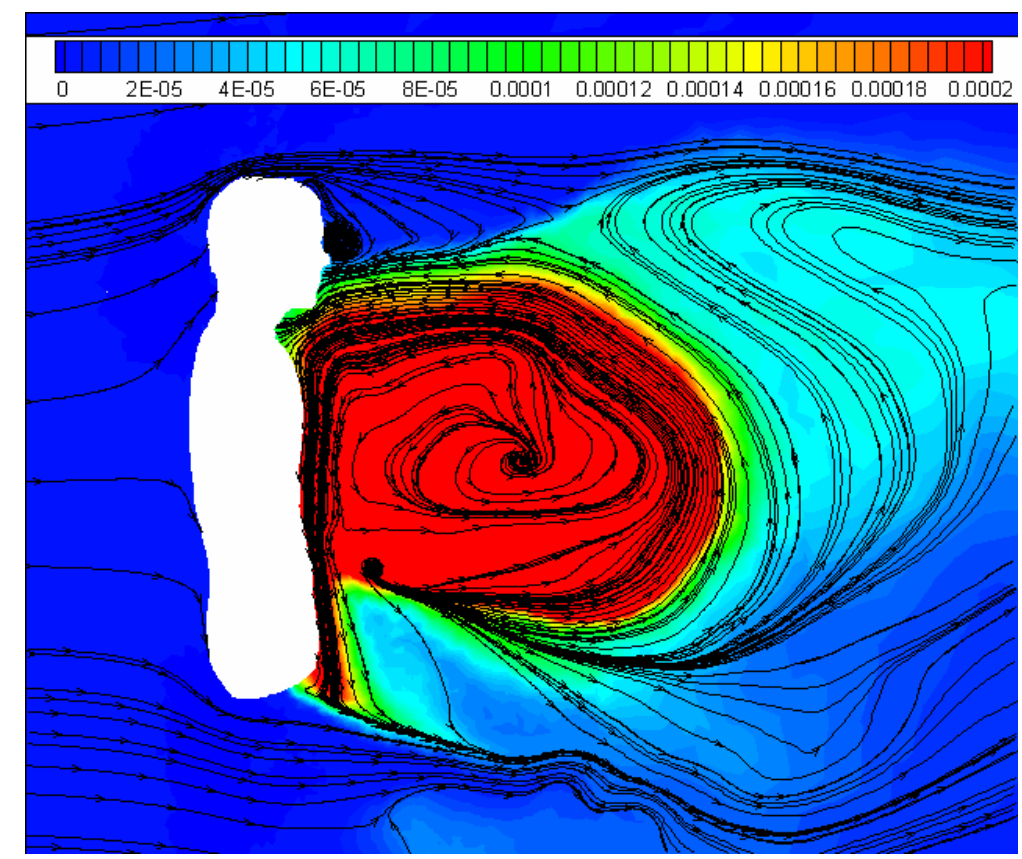

Figure 3-32 Instantaneous Pathlines and concentration contours in the middle plane at $t=620 \mathrm{~s}$ (concentration :kg/kgair)

The time evolution of the concentration at the breathing zone and the central chest are shown in Figure 3.33 and Figure 3.34, respectively. The mean and the standard deviation of the concentration at the breathing zone are $115 \mathrm{~kg} / \mathrm{kgair}(72 \mathrm{ppm})$ and $101 \mathrm{~kg} / \mathrm{kgair}(64 \mathrm{ppm})$, respectively. Comparing to the concentration predicted with the RNG and low-Reynolds-number RNG models, it is seen that the averaged concentration in the breathing zone calculated with LES lies in the mid range. More importantly, the LES results reveal that the concentration variation is so large that the instantaneous value cannot be used to estimate the averaged exposure level.

The power spectrum of the concentration in the breathing zone is calculated via Fourier transformation (Figure 3.35). The Strouhal number is defined by $S t=f D / U$. The typical frequency corresponding to a Strouhal number of $\mathrm{St}=0.047$ is $0.0235 \mathrm{~s}^{-1}$. The period is approximately $43 \mathrm{~s}$, which means the experimental sampling time (15 minutes) is sufficient for this case.

It should be noted that it takes a couple of weeks to finish the LES simulation for just 15 minutes of real time. LES costs 20 times more than the steady RANS simulation with RNG model in this case. 


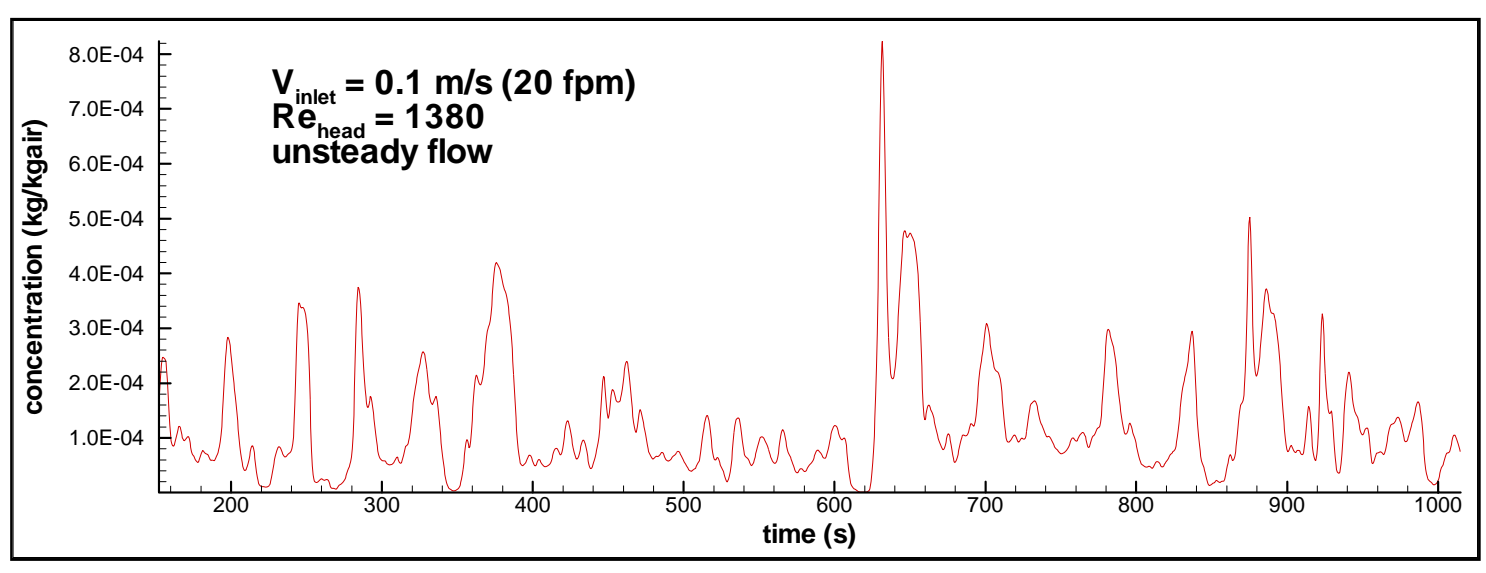

Figure 3-33 Concentration at the breathing zone changing with time (unheated body; LES)

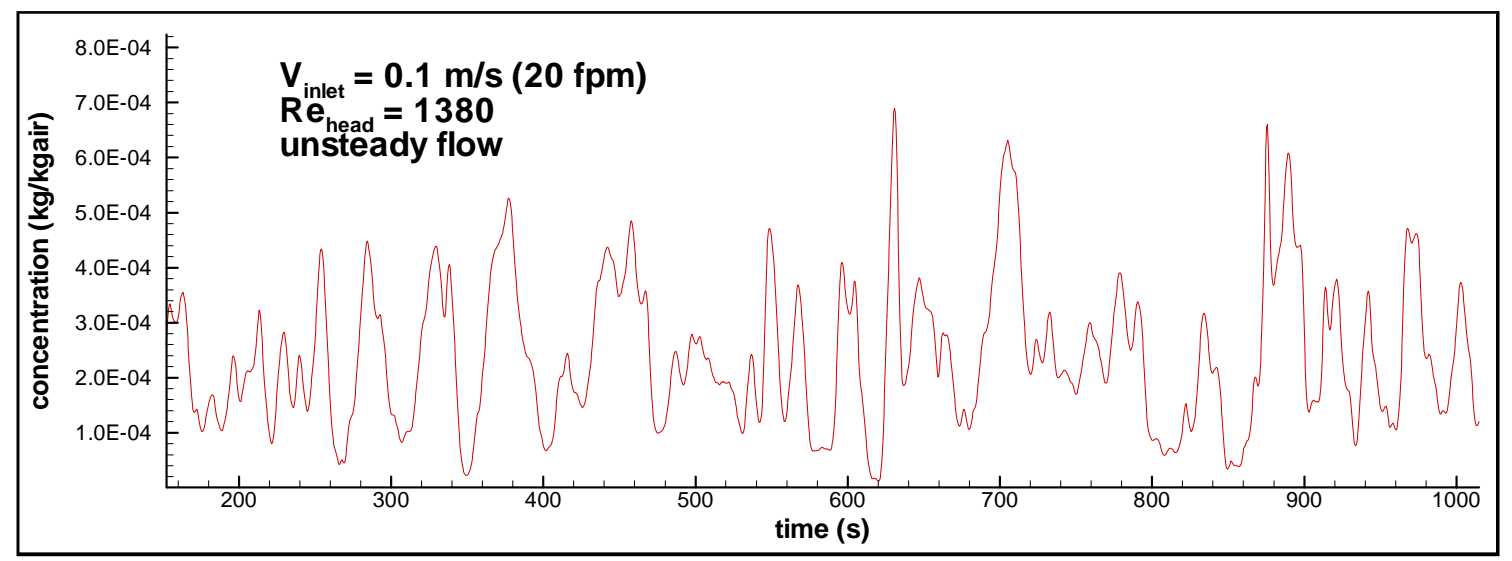

Figure 3-34 Concentration at the central chest changing with time (unheated body; LES) 


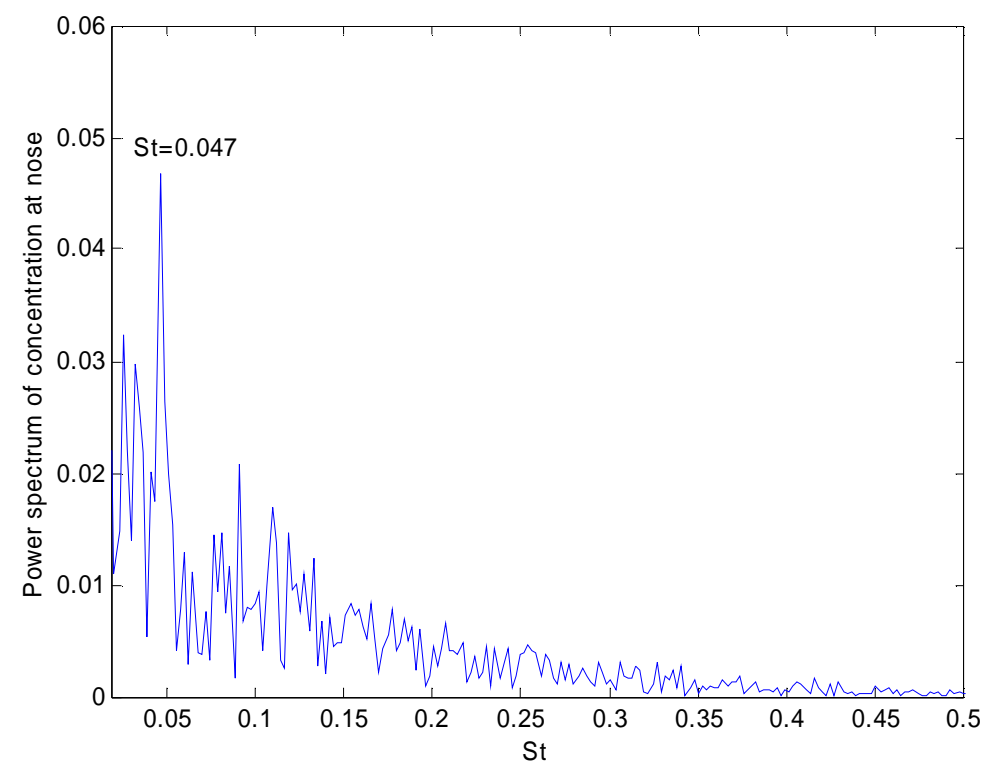

Figure 3-35 The power spectrum of concentration at the breathing zone

\subsection{Summary}

In this study, critical factors including body shape, ventilation intensity, free stream turbulence, body heat, and flow unsteadiness were scrutinized with respect to their effect on the air flow and gaseous pollutant transport in a wind tunnel when the body faces towards downstream of the flow and pollutant is released from a hand hold source pan. Part of the results were compared to experimental data (Guffey, 2005), which shows good agreement. An extensive grid convergence study was performed to determine the numerical uncertainty induced by finite numerical grid size. The overall discretization uncertainty is in the range of $5-10 \%$.

The influence of the shape of the human body used on worker exposure to contaminants was studied. It was observed to have a major impact on the flow-field and, consequently, on the concentration field. In fact, this study showed that the predictions with a rounded body, which is one of the closest approximation of a human body used in simulations in the literature (to the best of the author's knowledge), resulted in much lower predicted concentration levels. This suggests during modeling, oversimplified body shapes should not be preferred for worker exposure assessment. Specific details of rounded body may have a significant influence on the exposure and should be considered in certain circumstances. This presents a 
great challenge to CFD modelers as every worker may exhibit a significant variation on body shape depending on what clothes are worn and factors related to many other body size and shape.

It has to be noted that the heat flux from the body has a significant impact on predicted flow-field and concentration levels, so that it can not be simply neglected especially when the convection induced by the buoyancy dominates. The effect of the ventilation intensity on the exposure levels with an unheated body exhibits a simple expected pattern, i.e., as ventilation intensifies, exposure declines. However, for a heated body, the ventilation intensity region for maximum exposure levels moves to approximately $30-50$ fpm $(0.15-0.25 \mathrm{~m} / \mathrm{s})$ from $10 \mathrm{fpm}(0.05 \mathrm{~m} / \mathrm{s})$ for an unheated body. As the free stream turbulence increases, the exposure levels decrease.

The Eulerian scalar transport model and the Lagrangian trajectory tracking method were compared in their ability to predict the concentrations around a human shaped body exposed to gaseous contaminants within the length of an arm's reach. It was seen that the Eulerian method exhibited a more diffusive nature than the Lagrangian method. However, the concentration predictions obtained with the Lagrangian method may converge with the Eulerian prediction if an adequate number of trajectories are tracked. Nevertheless, the results obtained from both predictions suggest that the concentrations measured at the lapel could be very different than the concentrations measured near the mouth (generally, significantly higher). The present study indicates that the latter will be significantly lower under the specific condition of the present simulations, particularly in the absence of heat flux from the body. However, this conclusion should not be generalized to the cases with uniform concentration distribution, which may resulte from by a non-local source or even from the motion of the worker.

On another note, it was demonstrated that the numerical mesh size and its distribution have significant effects on the quality of predictions. It is recommended that in exposure studies grid convergence of CFD simulations should be assessed in order to reduce and quantify the degree of discretization errors. 


\section{Low-Reynolds-number cubic k- $\varepsilon$ turbulence model}

Advanced turbulence models have been briefly introduced in chapter 2. A cubic low-Reynoldsnumber turbulence model of Craft et al. (1996) has been selected for further study. This chapter will focus on how this cubic model is derived and what advantages it has over the linear eddy viscosity turbulence models. To do this, the features of the flow around the human body are first summarized, which is followed by the formulation and the defects of the linear eddy viscosity turbulence models. Finally, the cubic lowReynolds-number turbulence model will be presented.

\subsection{Introduction}

As seen from the LES study and the experiments, the complex unsteady flow around the human body is featured with separation, reattachment, buoyancy effects, vortex shedding and the interaction of the vortices.

If attention is initially restricted to statistically steady conditions, the flow feature of the primary concern is the recirculation which corresponds to the separation and the reattachment. All are affected by the details of the turbulence structure. The response of the decelerating boundary layer to the adverse pressure gradient, and hence the separation, is dictated by the turbulent shear stress as well as the normal stresses. The shear stress is sensitive to normal straining and streamline curvature, and this linkage occurs via the normal stresses which are themselves sensitive to streamwise, shear and curvature-related straining. Near a wall, however, turbulence is highly anisotropic. This anisotropy, induced by a combination of different production rates for the normal stresses and the kinematic blocking effect of the wall, needs to be correctly captured if the shear stress is to be evaluated accurately. As the flow approaches separation, the behavior of the near-wall layer departs drastically from any universal law of the wall, and its detailed structure must be resolved, including that of the semi-viscous sublayer in which viscosity affects turbulence. Similar comments apply to recirculation and reattachment regions. Thus, the turbulent normal stresses are dynamically active, and the turbulence is sensitive to streamline curvature. A further complication in 
relation to reattachment is that the turbulent state in this region depends sensitively on the evolution of turbulence in the separated shear layer about to reattach.

The buoyancy due to the heat flux from the human body has an important effect on the mean flow field as well as the turbulence structure. To predict the influence of buoyancy, the temperature field needs to be accurately calculated. This can be done only if the viscous sublayer is resolved correctly. This, however, makes the wall function approach for the temperature useless since there is no universal law for such complex three dimensional flows (Hanjelic, 2002).

Unsteadiness introduces a fundamental and profound uncertainty into the RANS framework. Reynolds-averaging, whether ensemble- or time-based, presupposes that the flow is statistically steady. At the very least, the time-scale associated with the organized unsteady motion must be substantially larger than the time-scale of the turbulent motion - or, in other words: the two time-scales must be well separated. This condition may be satisfied in low-frequency dynamics vortex shedding. On the assumption that conventional turbulence models may be applied to (a restricted range of) unsteady flows, the level of closure may have important implications for predictive accuracy. Unsteadiness increases the rate of change of all flow quantities and results, in particular, in a phase lag between the mean motion and the turbulence quantities. A relevant parameter to consider is the ratio of the time scales of the mean motion and the turbulence, i.e., $\Omega \mathrm{k} / \varepsilon$, where $\mathrm{k}$ is the turbulence energy and $\varepsilon$ is its rate of dissipation and $\Omega$ is the frequency of the mean motion. As the wall is approached, this ratio declines rapidly, given a fixed frequency of the mean-flow oscillation(Lien and Leschziner, 1994). The implication is that the turbulence structure close to the wall will adjust rapidly to the external oscillation, so that a quasi-steady turbulence closure would be expected to be adequate in this region. However, towards the outer part of the boundary layer, the turbulence field will not respond quickly to the oscillation and the turbulent stresses would tend to freeze, with consequent phase shift between the mean motion and the turbulence field. To take this into account, a turbulence model needs to account for stress transport. Hence, unsteady flows can, in general, be expected to benefit from non-linear or second-moment closure which entails a reduced level of reliance on equilibrium assumptions. 


\section{Turbulence model categories}

There are three principal classes of models currently used in computations of airflow and pollutant transport:

- $\quad$ Linear eddy-viscosity models (LEVM);

- $\quad$ Non-linear eddy-viscosity models (NLEVM);

- $\quad$ Reynolds-stress models (RSM).

Some models do not fall neatly into any one of the above categories, straddling two categories or

containing elements from more than one category. For instance, the " $\bar{v}{ }^{2}-f$ " model of Durbin (1991) is essentially a LEVM, but incorporates a simplified transport equation for the normal stress perpendicular to streamlines (or the wall), which serves as the turbulent velocity scale in the eddy-viscosity, in preference to the turbulence energy.

Some other models exist, but have not been used to any significant extent for practical computations. These include

- Various models derived from two-point correlation functions (eg Cambon and Scott, 1999)

- $\quad$ The "Structure-Based Model" (Kassinos et al. 2000);

- Multi-scale models which are based on the partitioning of the turbulence-energy spectrum, each partition associated with a different size range of eddies (eg. Schiestel, 1987; Wilcox, 1988).

Within any one of the above major categories, there are dozens of variants, and the LEVM category, being the simplest, contains several sub-categories and is typically heavily populated with model variations, many differing from other forms by the inclusion of minor (though sometimes very influential) 'correction terms', or different functional forms of model coefficient or even through slight differences in the numerical values of model constants. To a considerable degree, this proliferation reflects a trend to adopt or adhere to simple turbulence models for the modeling task at hand and then to add 'patches' so as 
to cure specific ills for specific sets of conditions. Other not unimportant contributory factors are insufficiently careful and excessively narrow validation, yielding misleading statements on the predictive capabilities of existing models, and the fact that publishing a new model, rather than a study quantifying the capabilities of an existing model, is much easier.

\section{Linear eddy-viscosity model}

All models in this category are based on the following linear stress-strain relationship:

$$
-\rho \overline{u_{i} u_{j}}=\mu_{t}\left(\frac{\partial U_{i}}{\partial x_{j}}+\frac{\partial U_{j}}{\partial x_{i}}-\frac{2}{3} \frac{\partial U_{k}}{\partial x_{k}} \delta_{i j}\right)-\frac{2}{3} \rho k \delta_{i j}
$$

in which $\mu_{t}$ is the eddy viscosity, $\mathrm{U}_{\mathrm{i}}$ is the velocity vector, $\mathrm{x}_{\mathrm{i}}$ are the spatial coordinate, $\rho$ is the density, $\delta_{i j}$ is the Knoecker delta - a unit tensor with unit diagonal and zero off-diagonal elements and $\mathrm{k}$ is the (kinematic) turbulence energy. The right-most term in Eq. (4.1) is required to ensure that the normal stresses sum up to $2 \mathrm{k}$ in zero strain. The eddy viscosity is usually determined by a velocity scale $\mathrm{k}^{1 / 2}$ and a length scale( eg. $\varepsilon$ in the k- $\varepsilon$ two equation model). The eddy viscosity is expressed as

$$
v_{t}=C_{\mu} \frac{k^{2}}{\varepsilon}
$$

The differential equation adopted for $\mathrm{k}$ is

$$
\frac{\partial k}{\partial t}+\frac{\partial U_{j} k}{\partial x_{j}}=\frac{\partial}{\partial x_{j}}\left(\mu+\frac{\mu_{t}}{\sigma_{k}}\right) \frac{\partial k}{\partial x_{j}}+P_{k}-\varepsilon
$$

This equation arises upon a summation of the exact Reynolds-stress-transport equations for the normal stresses and a replacement of the exact diffusion term (which contains unknown triple correlations $\overline{u_{j} u_{k} u_{k}}$ and $\overline{u_{j} p}$ ) by a gradient-diffusion model, with $\sigma_{k}$ being the turbulent Prandtl/Schmidt number for $\mathrm{k}$. The foundation for a closed equation for the length scale is an exact transport equation that can be derived for the dissipation rate 


$$
\varepsilon=\overline{\frac{\partial u_{i}}{\partial x_{j}} \frac{\partial u_{i}}{\partial x_{j}}}
$$

or any other quantity of the form $\zeta=k^{a} \varepsilon^{b}$. This derivative involves a laborious combination of derivatives of the Navier-Stokes equations and results in an equation of the form

$$
\frac{\partial \varepsilon}{\partial t}+\frac{\partial U_{j} \varepsilon}{\partial x_{j}}=\sum_{k=1,4} P_{\varepsilon, k}+\sum_{l=1,3} D_{\varepsilon, l}-\varepsilon_{\varepsilon}
$$

which contains numerous groups of correlations interpretable as production $\left(\mathrm{P}_{\varepsilon}\right)$, diffusion $\left(\mathrm{D}_{\varepsilon}\right)$ and destruction $\left(\varepsilon_{\varepsilon}\right)$. The left-hand side represents convection and arises precisely as written in Eq. (4.5). Termby-term modeling of this equation has been attempted by Rodi and Mansour (1993) on the basis of DNS data, but has not been really productive in terms of creating a working model superior to more established forms. The first practical forms of a dissipation equation were proposed by Harlow (1968) and Hanjalic (1970). The basic form (Johns and Launder, 1972) used in conjunction with the k-equation (Eq. 4.3) and expression (Eq. 4.2) for the eddy viscosity is:

$$
\frac{\partial \varepsilon}{\partial t}+\frac{\partial U_{j} \varepsilon}{\partial x_{j}}=C_{\varepsilon 1} \frac{\varepsilon}{k} P_{k}+\frac{\partial}{\partial x_{j}}\left(\left(\frac{v_{t}}{\sigma_{\varepsilon}}+v\right) \frac{\partial \varepsilon}{\partial x_{j}}\right)-C_{\varepsilon 2} \frac{\varepsilon^{2}}{k}
$$

Its applicability to low-Reynolds-number near-wall flows necessitates the introduction of damping functions and additional terms that insure the correct near-wall behavior, and it is the need for this extension that has spawned numerous model variations (Johns and Launder, 1972; Launder and Sharma, 1974; Hoffman, 1975; Lam and Bremhorst, 1981; Chien, 1982; Nagano and Hishida, 1987; Myong and Kasagi, 1990; So et al, 1991; Orszag et al, 1993; Kawamura and Kawashima, 1994; and Lien and Leschziner, 1994). It is informative, prior to reviewing these extended models, to comment on a few fundamental aspects of modeling the dissipation rate.

First, the Taylor-series analysis can help in understanding the near wall behavior of $\varepsilon$. The turbulent velocity components, in terms of wall distance, can be written as:

$$
u_{k}=a_{k}(t) y+b_{k}(t) y^{2}+c_{k}(t) y^{3}+\cdots \quad\left(u_{k}=u, v, w\right)
$$


The Taylor-series analysis can readily be applied to Eq. (4.4) to show that the wall-asymptotic behavior of the dissipation is $\varepsilon=\mathrm{O}(1)$. At the wall, the rate of dissipation must be balanced by viscous diffusion of turbulence energy,

$$
\left.\varepsilon\right|_{y \rightarrow 0}=\left.\mu \frac{\partial^{2} k}{\partial y^{2}}\right|_{y \rightarrow 0}
$$

In fact, DNS simulations for channel flow by Moser et al. (1999) and others show that $\varepsilon$ reaches a maximum at the wall. The fact that the wall value is unknown, but needs to be determined via the compatibility condition (Eq. 4.8) is numerically disadvantageous and has led to the adoption of the "homogeneous" dissipation

$$
\tilde{\varepsilon}=\varepsilon-2 v\left(\frac{\partial k^{1 / 2}}{\partial x_{j}}\right)^{2}
$$

as the dependent variable, with its wall-asymptotic variation arising as $\mathrm{O}\left(\mathrm{y}^{2}\right)$. This is the variable used in the models of Jones and Launder (1972), and Launder and Sharma (1974).

Although they may be appropriate for flows in which a single shear stress is the dominant dynamic link between turbulence and the mean-flow, all are afflicted by the defects:

- $\quad$ They do not resolve normal-stress anisotropy;

- $\quad$ They do not account for transport of stresses (by convection and diffusion) but link rigidly the stresses to the strain rates;

- $\quad$ They over-estimate the stresses at high strain rates;

- They do not respond correctly to curvature strain, normal straining and rotation (though ad-hoc patches help in some circumstances);

- They misrepresent, when used in conjunction with eddy-diffusivity/gradient-diffusion approximations, the heat fluxes, except for the flux component normal to simple shear layers with a dominant cross-layer temperature gradient. 
An alternative, much simpler route is available for approximating the Reynolds stresses which adopts an algebraic connection between stress and strain - albeit not a linear relationship. Such relationships may be arrived at by simplifying stress-transport models (so-called algebraic stress models, ASMs) but, in view of the current limitations of such schemes alluded to above, it is best to regard them simply as conjectured generalizations of the eddy-viscosity approach, containing quadratic and, occasionally, higher-order products of the strain and vorticity tensors. The earliest schemes go back to the 1970s (Pope, 1975), although, in the last few years, there have been concerted efforts by many different groups world wide.

Quadratic stress-strain relationships have been proposed by a number of recent studies, such as Speziale (1987), Nisizima and Yoshizawa (1987), Rubinstein and Barton (1990), Myong and Kasagi (1990) and Shih, et al (1993). All of these studies arrived at the empirical coefficients by considering the prediction of shear stress in a simple shear and one other complex flow (or some other feature of a simple shear-such as the normal stress level - that can not be mimicked with a linear scheme).

However, Craft et al. (1996) argued that there seems be little agreement between them on coefficient values, which implies that, at quadratic level, only slightly greater generality is achievable than with the usual linear eddy-viscosity model. In particular, the effects of streamline curvature and swirl on the turbulent stresses cannot be adequately accounted for at this level. This is the reason he proposed a cubic eddy-viscosity model. The cubic model used in this study is based on CLS model (Craft et al. 1996) and some modifications are introduced.

\subsection{Methodology for cubic eddy-viscosity model}

In this turbulence model, Reynolds stresses are obtained via the constitutive stress-strain relation: 


$$
\begin{aligned}
& \overline{u_{i} u_{j}}=\frac{2}{3} k \delta_{i j}-v_{t} S_{i j}+C_{1} \frac{v_{t} k}{\bar{\varepsilon}}\left(S_{i k} S_{k j}-\frac{1}{3} S_{k l} S_{k l} \delta_{i j}\right) \\
& +C_{2} \frac{v_{t} k}{\bar{\varepsilon}}\left(\Omega_{i k} S_{k j}+\Omega_{j k} S_{k i}\right)+C_{3} \frac{v_{t} k}{\bar{\varepsilon}}\left(\Omega_{i k} \Omega_{j k}-\frac{1}{3} \Omega_{l k} \Omega_{l k} \delta_{i j}\right) \\
& +C_{4} \frac{v_{t} k^{2}}{\bar{\varepsilon}}\left(S_{k i} \Omega_{l j}+S_{k j} \Omega_{l i}\right) S_{k l}+C_{5} \frac{v_{t} k^{2}}{\bar{\varepsilon}^{2}}\left(\Omega_{i l} \Omega_{l m} S_{m j}+S_{i l} \Omega_{l m} \Omega_{m j}-\frac{2}{3} S_{l m} \Omega_{m n} \Omega_{n l} \delta_{i j}\right) \\
& +C_{6} \frac{v_{t} k^{2}}{\bar{\varepsilon}^{2}} S_{i j} S_{k l} S_{k l}+C_{7} \frac{v_{t} k^{2}}{\bar{\varepsilon}^{2}} S_{i j} \Omega_{k l} \Omega_{k l}
\end{aligned}
$$

where $S_{i j}$ and $\Omega_{i j}$ are strain and vorticity rate tensors, respectively, given by

$$
\begin{gathered}
S_{i j}=\frac{\partial U_{i}}{\partial x_{j}}+\frac{\partial U_{j}}{\partial x_{i}} \\
\Omega_{i j}=\frac{\partial U_{i}}{\partial x_{j}}-\frac{\partial U_{j}}{\partial x_{i}}
\end{gathered}
$$

The turbulent viscosity, $v_{t}$, is obtained from

$$
v_{t}=C_{\mu} f_{\mu} \frac{k^{2}}{\bar{\varepsilon}}
$$

where the variable $\bar{\varepsilon}$ is the isotropic dissipation rate which can be related to the real dissipation rate through:

$$
\bar{\varepsilon}=\varepsilon-2 v\left(\frac{\partial \sqrt{k}}{\partial x_{j}}\right)^{2}
$$

Lee et al. (1990) found, from a comparative DNS study of the appearance of eddy structures in homogeneous shear flows and near-wall turbulence, that the strain invariant was mainly responsible for the streaky structure in the viscous "buffer" region near a wall rather than the turbulent Reynolds number. Craft et al. (1996) argued that the near-wall behavior of turbulence, although strongly affected by viscosity, cannot be adequately characterized in terms of a viscosity-based parameter alone. The strain parameter, $\mathrm{S}$, provides a possible additional parameter. 
Optimization over a wide range of flows by Craft et al. (1996) has resulted in the following expressions for $C_{\mu}$ and $f_{\mu}$ :

$$
\begin{aligned}
& C_{\mu}=\frac{0.3}{1+0.35 \eta^{1.5}}\{1-\exp [-0.36 \exp (0.75 \eta)]\} \\
& f_{\mu}=1-\exp \left[-\left(\overline{R_{t}} / 90\right)^{1 / 2}-\left(\overline{R_{t}} / 400\right)^{2}\right]
\end{aligned}
$$

where $\eta=\max (\bar{S}, \bar{\Omega})$ and $\bar{R}_{t}=k^{2} / v \overline{\mathcal{E}}$ is the local turbulent Reynolds number.

The functional form of $C_{\mu}$ has thus been tuned so that, in a simple homogeneous shear flow at high Reynolds number, good agreement with experimental and direct numerical simulation data is obtained for the variation of $\overline{u^{\prime} v^{\prime}} / k$ with strain rate $\mathrm{S}$, as shown in Figure 4.1. The nonlinear elements allow good predictions to be obtained also for the normal stress anisotropies. Note that the linear eddy-viscosity model gives $\mathrm{a}_{11}=\mathrm{a}_{22}=0$, and $\mathrm{a}_{12}=0.09 \mathrm{~S}$, neither can the quadratic models predict the correct variation of $\mathrm{a}_{\mathrm{ij}}$ with $\mathrm{S}$ in this simple shear flow $\left(a_{i j}=\overline{u_{i} u_{j}}-\frac{2}{3} k \delta_{i j}\right)$.

An additional Reynolds number-dependent damping term $f_{\mu}$ is still required for near-wall flows, or for low Re flows, but its influence is considerably less than that used in the linear eddy-viscosity models, because now a substantial amount of the near-wall strain-related damping is provided by the functional form of $C_{\mu}$.

The model coefficients, $\mathrm{C}_{1}-\mathrm{C}_{7}$, have been calibrated by Craft et al. (1996), by reference to several flows, including homogeneous shear flows, swirling flows and curved channel flows. The values of these coefficients are given as

$$
\mathrm{C}_{1}=-0.1, \mathrm{C}_{2}=0.1, \mathrm{C}_{3}=0.26, \mathrm{C}_{4}=-10 \mathrm{C}_{\mu}^{2}, \mathrm{C}_{5}=0, \mathrm{C}_{6}=-5 \mathrm{C}_{\mu}^{2}, \mathrm{C}_{7}=5 \mathrm{C}_{\mu}^{2}
$$




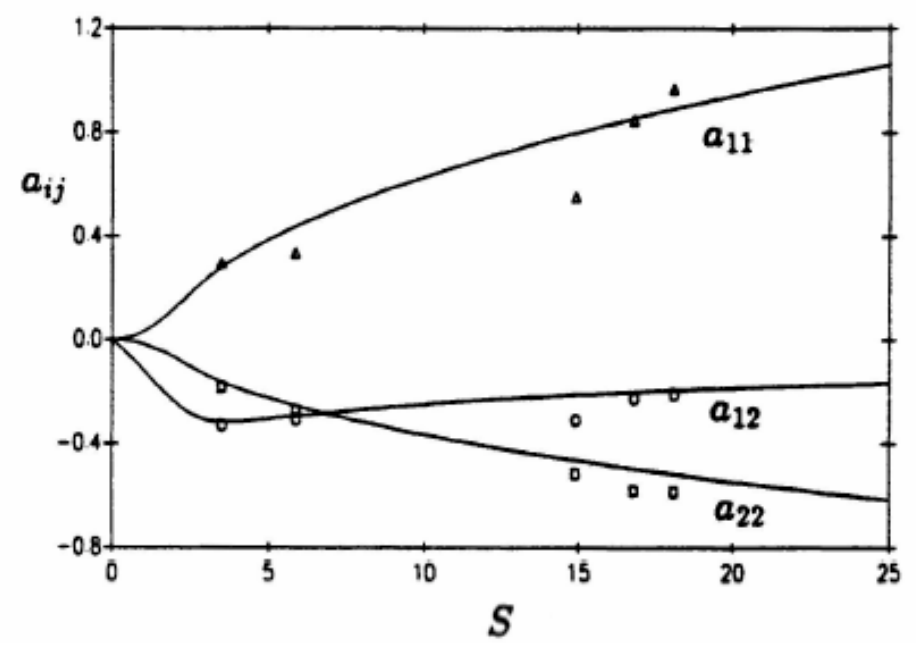

Figure 4-1 The variation of $\overline{u_{i}^{\prime} u_{j}^{\prime}} / k$ with strain rate (Craft et al. 1996)

To obtain $v_{t}$, transport equations for the turbulent kinetic energy, k, and its dissipation rate, $\bar{\varepsilon}$, are solved. The transport equation for turbulent kinetic energy is written as:

$$
\frac{\partial k}{\partial t}+U_{j} \frac{\partial k}{\partial x_{j}}=\frac{\partial}{\partial x_{j}}\left[\left(v+\frac{v_{t}}{\sigma_{k}}\right) \frac{\partial k}{\partial x_{j}}\right]+P_{k}-\bar{\varepsilon}-2 v\left(\frac{\partial \sqrt{k}}{\partial x_{i}}\right)^{2}
$$

The dissipation rate of turbulent kinetic energy is obtained by solving the equation:

$$
\frac{\partial \bar{\varepsilon}}{\partial t}+U_{j} \frac{\partial \bar{\varepsilon}}{\partial x_{j}}=\frac{\partial}{\partial x_{j}}\left[\left(v+\frac{v_{t}}{\sigma_{\varepsilon}}\right) \frac{\partial \bar{\varepsilon}}{\partial x_{j}}\right]+C_{\varepsilon 1} f_{1} \frac{\bar{\varepsilon}}{k} P_{k}-C_{\varepsilon 2} f_{2} \frac{\bar{\varepsilon}^{2}}{k}+E+S_{\bar{\varepsilon}}
$$

and $P_{k}$ is the generation rate of turbulent kinetic energy obtained from:

$$
P_{k}=-\overline{u_{i} u_{j}} \frac{\partial U_{i}}{\partial x_{j}}
$$

The damping function $f_{1}$ and $f_{2}$ are given by:

$$
\begin{aligned}
& f_{1}=1 \\
& f_{2}=1-0.3 \exp \left(-\bar{R}_{t}^{2}\right)
\end{aligned}
$$


The near wall source term $E$ is expressed as:

$$
E= \begin{cases}C_{e} \frac{\bar{S} v_{t} k^{2}}{\bar{\varepsilon}}\left(\frac{\partial^{2} U_{i}}{\partial x_{k} \partial x_{l}}\right)^{2} & \bar{R}_{t} \leq 250 \\ 0 & \bar{R}_{t}>250\end{cases}
$$

The model constants are given as

$$
\begin{gathered}
C_{\varepsilon 1}=1.44, \quad C_{\varepsilon 2}=1.92 \quad, \quad \sigma_{k}=1.0 \quad, \quad \sigma_{\varepsilon}=1.3 \quad, \quad C_{e}=0.0022 \quad \text { and } \\
\mathrm{C}_{\mu}=\min \left[0.09, \frac{12}{1+3.5 \eta+\mathrm{f}_{\mathrm{RS}}}\right] \quad \text { with } f_{R S}=0.235[\max (0, \eta-3.333)]^{2} \exp \left(-\bar{R}_{t} / 400\right) \quad \text { and } \\
\eta=\max (\bar{S}, \bar{\Omega}) \text { where } \bar{S}=\frac{k}{\varepsilon} \sqrt{\frac{1}{2} S_{i j} S_{i j}} \text { and } \bar{\Omega}=\frac{k}{\varepsilon} \sqrt{\frac{1}{2} \Omega_{i j} \Omega_{i j}} .
\end{gathered}
$$

In separated flows, the near-wall length-scale becomes too large, resulting in excessively high levels of near-wall turbulence. To overcome this behavior, Yap (1987) introduced an extra source term into the dissipation rate equation which is based on the wall distance y:

$$
S_{\varepsilon}=Y a p=C_{y} \frac{\bar{\varepsilon}^{2}}{k} \max \left[\left(l / l_{e}-1\right)\left(l / l_{e}\right)^{2}, 0\right]
$$

where $C_{y}=0.0022, l$ is the turbulent length-scale, $k^{3 / 2} / \bar{\varepsilon}$, the equilibrium length-scale $l_{e}=2.55 y$ and $\mathrm{y}$ is the distance normal to the wall.

\subsection{Implementation in DREAM code}

The current choice for RANS simulations in the DREAM code (Celik and Badeau, 2003) is the hybrid scheme which has accuracy in between the first order and the second order, as it toggles between upwind differencing and central differencing schemes depending on the value of the cell Peclet number. However, in the current implementation, the grid is so generated that the Peclet number is smaller than 2.0 at least in those important regions (e.g. the recirculation region for the backward facing step flow), which 
ensures that the central differencing scheme will be utilized. Also, this indicates that the leading truncation error of the scheme is now proportional to the square of grid spacing.

The projection method (Kim and Moin, 1985), also known as the fractional step approach, was used to solve the incompressible Navier-Stokes equations. The key feature of the projection method is a time-splitting discretization scheme which decouples the computation of velocity and pressure. In the first step, an intermediate velocity field is computed using the momentum equation and ignoring the incompressibility constraint. In the second step, the intermediate velocity is projected to the space of divergence free vector fields to get the next update of velocity and pressure. This procedure is more efficient than solving a coupled system of Stokes equations for velocity and pressure which would arise from a straightforward time discretization of the Navier-Stokes equations. For details, see Celik and Badeau (2003). 


\section{Application of low-Reynolds-number cubic k- $\varepsilon$ turbulence model}

Turbulent channel flow, backward facing step flow, and flow past a square cylinder are selected to evaluate the performance of the cubic low-Reynolds number model. Turbulent channel flow calculations can provide information on how this model predicts anisotropy. Turbulent flow over a backward facing step is a widely used bench-mark to investigate the performance in complex separated and reattaching turbulent flows. Flow past a square cylinder is chosen since it can somehow mimic the flow past a simplified human body.

\subsection{Turbulent Channel Flow}

Fully developed channel flow has been extensively studied experimentally and numerically to increase the understanding of the mechanics of wall-bounded turbulent flow. Its geometric simplicity is attractive for both experimental and theoretical investigations of the complex turbulence interactions near a wall.

The Reynolds number based on the channel half height $\delta$ and the mean centerline velocity $U_{c}$ for the present simulation is Re_ $\delta=7890$ (Ercoftac Database, 2005), (a Reynolds number of 395 based on the friction velocity $\mathrm{u}_{\tau}$ ). A grid of $61 \mathrm{x} 91$ in $\mathrm{x}$ and $\mathrm{y}$ is used. The first mesh point away from the wall is at $\mathrm{y}^{+}=1$ in wall units. The superscript + indicates a non-dimensional quantity scaled by the wall variables, e.g. $y^{+}=y u_{\tau} / v$, where $v$ is the kinematic viscosity and $u_{\tau}=\left(\tau_{w} / \rho\right)^{1 / 2}$ is the wall shear velocity. The velocity and turbulent kinetic energy profiles at the inlet are imposed from DNS data. A periodic inlet boundary condition is used for the $\varepsilon$ at the inlet since it is not available from the DNS data. The cubic lowReynolds number model has been implemented in the DREAM code and applied to the cases in this chapter.

The profile of the streamwise velocity non-dimensionalized by the wall-shear velocity is shown in Figure 5.1. Also shown in the Figure 5.1 is the DNS data (Ercoftac Database, 2005). The computed results with the cubic low-Reynolds number turbulence model shows good agreement with the DNS data.

The turbulent normal stresses normalized by the wall-shear velocity are shown in Figure 5.2, and they are compared with the DNS data. The computed uu+ with the cubic low-Reynolds eddy-viscosity 
turbulence model is lower than the DNS data in the near wall region $(y+<40)$. The normal stresses in $y$ and $z$ directions are higher than the DNS data. The shape of these quantities is generally in good agreement with the DNS results.

The total turbulent kinetic energy is in good agreement with the DNS results (Figure 5.3). It should be noted here that there is no distinguishable difference in between the $\mathrm{k}$ calculated with the $\mathrm{k}$ equation in the turbulence model and the $\mathrm{k}$ computed with $k=\frac{1}{2}\left(\overline{u^{\prime 2}}+\overline{v^{\prime 2}}+\overline{w^{\prime 2}}\right)$ because the present model is a consistent RANS model (see Eq. 4.10).

It is only the shear stress which affects the mean velocity in this simple shear flow, and from Figure 5.4 this can be seen to be very well predicted. Although (unlike any linear eddy-viscosity model) the present model does give a separation between the normal stress components, the difference is not as large as is found in the near-wall region. Although this certainly is a deficiency, it is not a terribly serious one, because in this immediate near-wall region, it is the shear stress that governs the mean flow behavior. Of course, in the limit, where a flow impingement on to a wall is considered, the normal stresses must be influential. As shown by Craft et al. (1996), in this limit, very satisfactory normal-stress profiles are obtained.

Others' models (e.g., Apsley and Leschziner, 2000) add empirical functions of the distance to wall in Eq. 4.10. This term can account for the energy redistribution from $v_{\mathrm{rms}}$ and $\mathrm{w}_{\mathrm{rms}}$ to $\mathrm{u}_{\mathrm{rms}}$ so that the normal stresses obtained with their models match the DNS data pretty well for the channel flow. Nevertheless, the generalization of such a term on other applications is yet to be validated. 


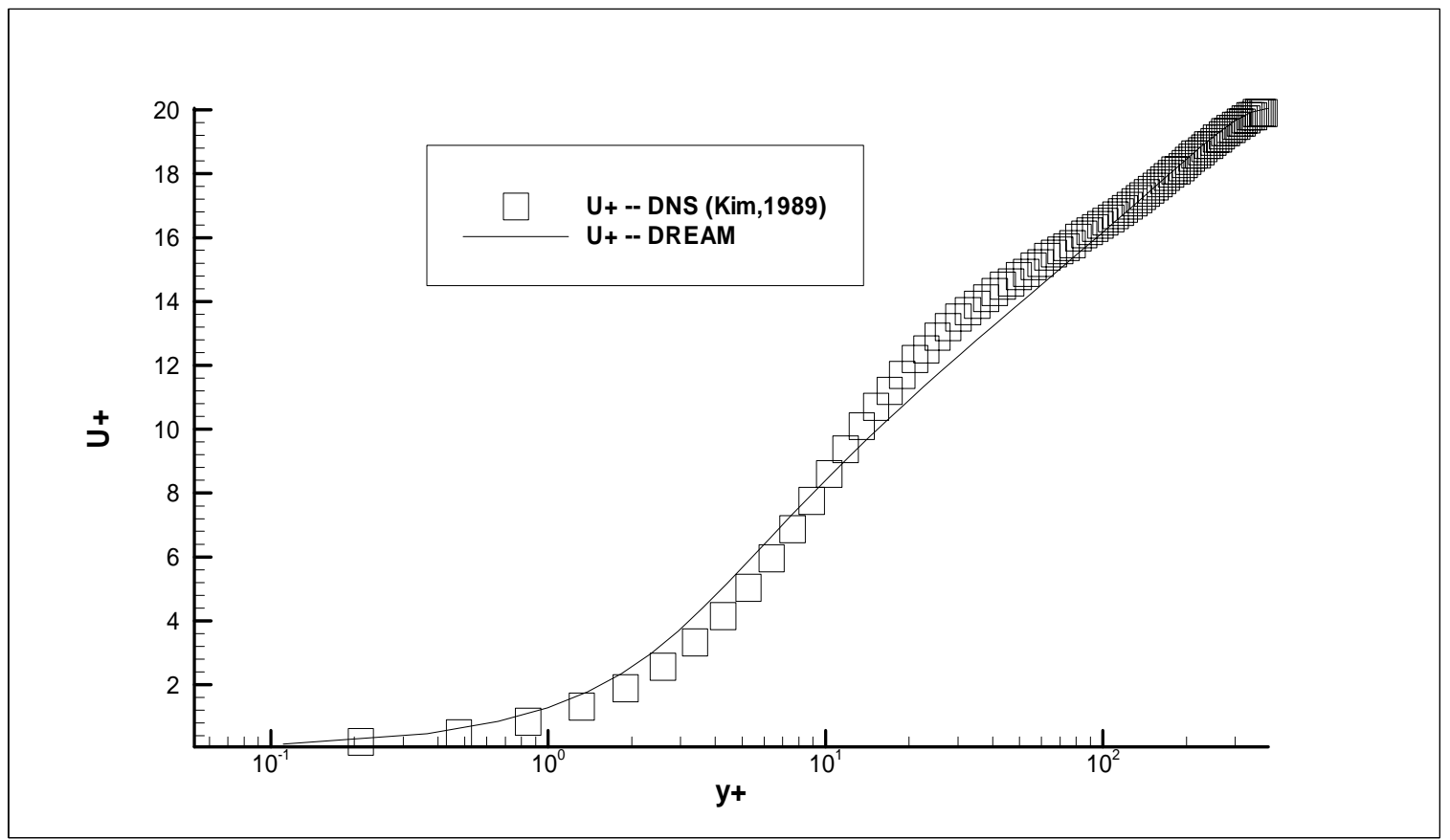

Figure 5-1 Profiles of the mean velocity for channel flow 


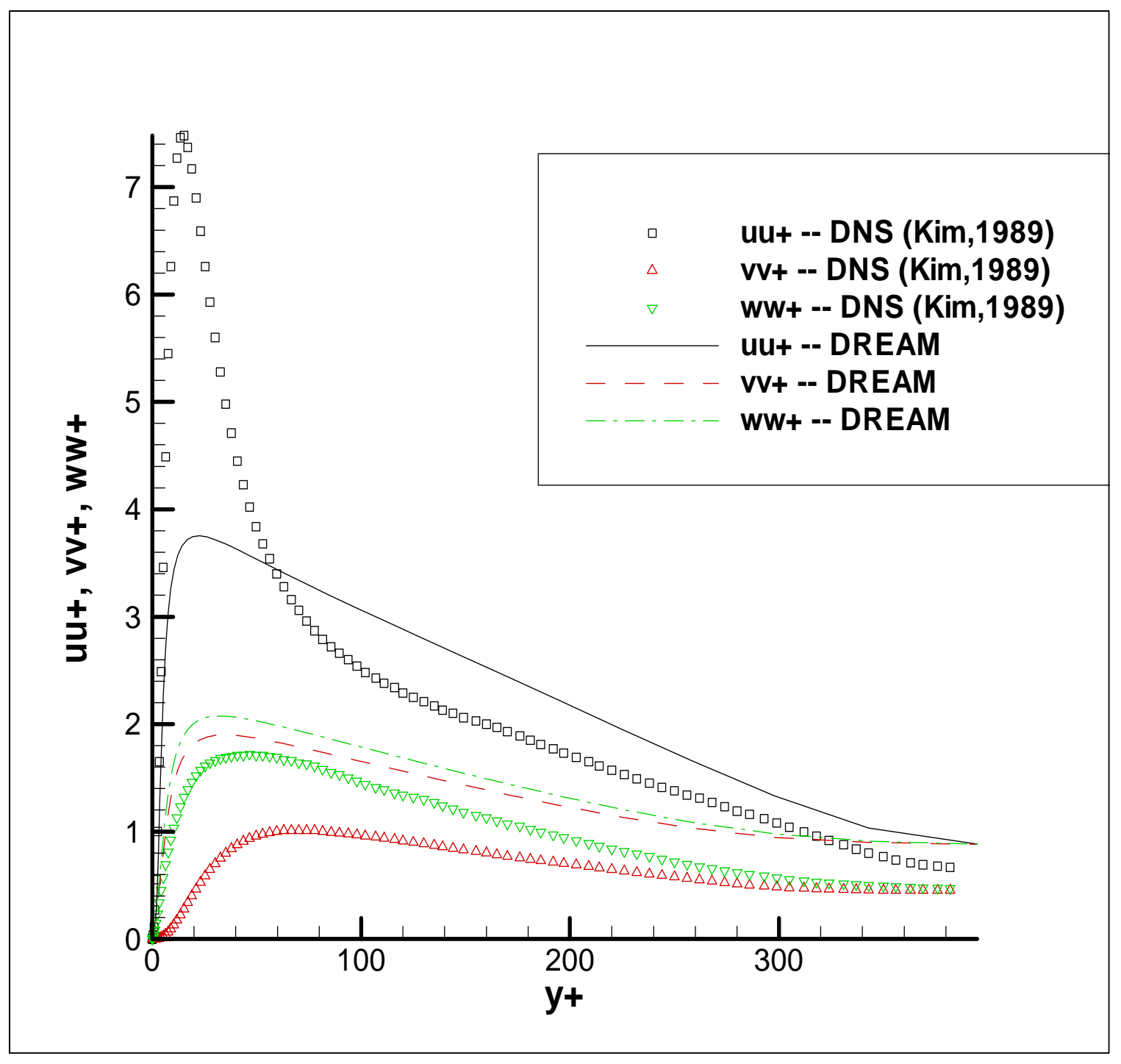

Figure 5-2 Profiles of turbulent normal stresses for channel flow 


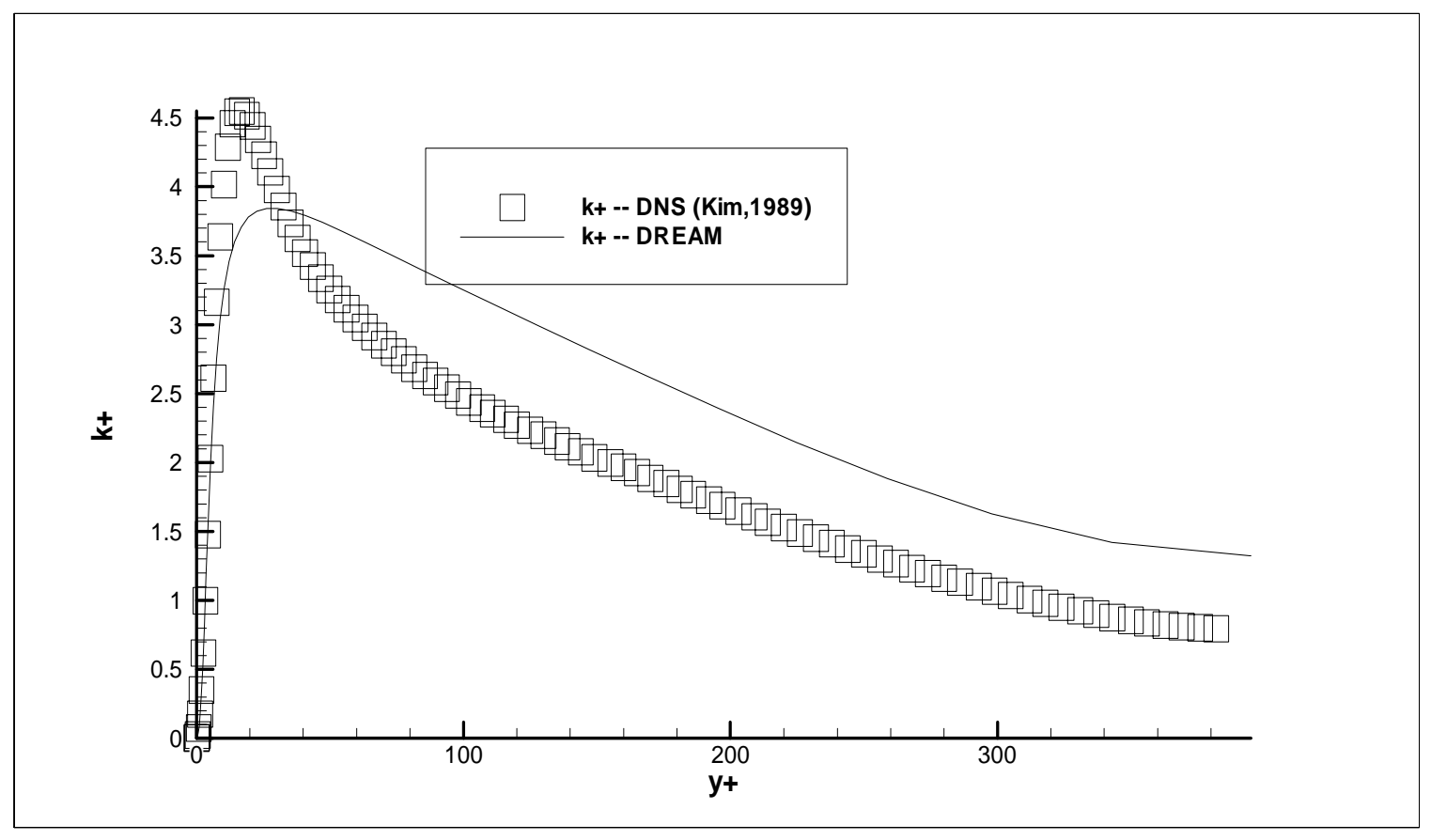

Figure 5-3 Profiles for the turbulent kinetic energy

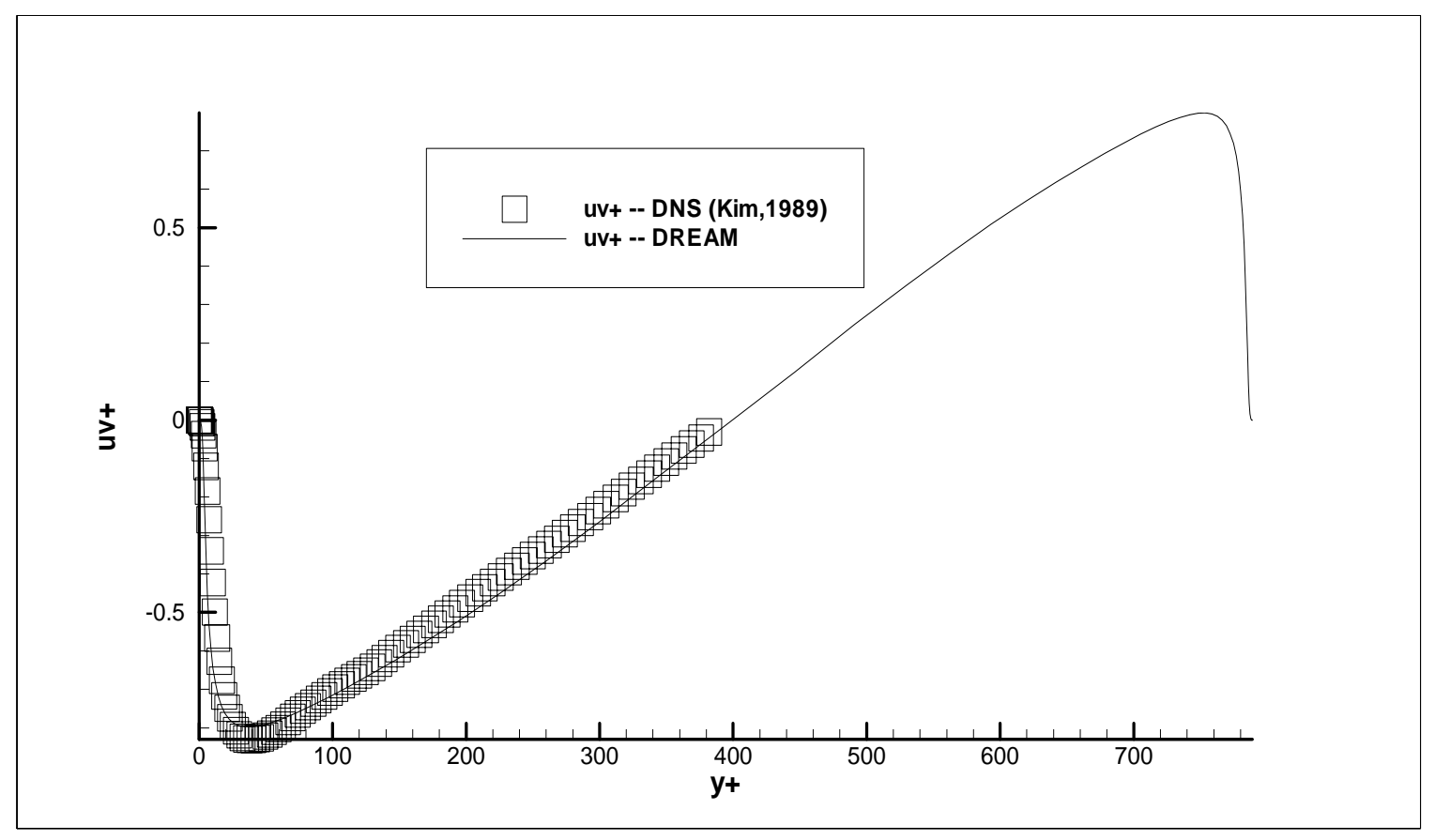

Figure 5-4 Profiles for the turbulent shear stress 


\subsection{Flow over a backward facing step}

Separation, recirculation, and reattachment are features encountered in numerous practical simulations. They occur whenever a fast-flowing fluid is required to bypass a blunt obstacle or whenever a confining wall undergoes a rapid change in orientation to form a strongly curved convex surface. Recirculation has profound consequences in relation to pressure recovery, pressure drag, wall friction and heat transfer characteristics. It is also a powerful generator of turbulence and hence mixing and losses. Separated flows have thus naturally been the subjects of many studies, both experimentally and computationally.

The objectives pursued in different studies have varied considerably. Frequently, the emphasis has been on understanding and capturing the separation process, particularly if it occurson a curved surface, on resolving the structure of the separated shear layer and the recirculation zone it envelops, on describing the location of reattachment and the dominant processes in the reattachment region, and on understanding and predicting the processes governing the flow recovery in the wake region following reattachment. All these issues can affect the transport of the gaseous contaminants in the wake of a worker. Before application to the worker exposure, the turbulence model should be validated to show that it can resolve these issues accurately.

The cubic low-Reynolds-number turbulence model is applied to the backward facing step flow of Le and Moin (1992). They have published extensive DNS data for backward facing step flow at Ercoftac database (ERCOFTAC, 2005). It should be noted that the case is usually called sudden expansion. Here it is named as backward facing step flow to be consistent with the Ercoftac database. The schematic view of the computational domain and the boundary conditions are shown in Figure 5.5. The Reynolds number, based on the step height $\mathrm{H}$ and the mean inlet free stream velocity $\mathrm{U}_{0}$, is $\mathrm{Re}_{\mathrm{H}}=5100$. The expansion ration is 1.2. The inlet velocity and turbulent kinetic energy are imposed from the DNS data. A periodic $\varepsilon$ inlet profile, which takes the $\varepsilon$ profile at $\mathrm{x} / \mathrm{H}=-3$, is specified as the inlet condition. It is assumed that $20 \mathrm{H}$ is long enough for the velocity to recover and the quantities at the outlet can maintain themselves. That's why the Neumann boundary condition is used at the outlet. It should be noted that the integrated mass conservation is also imposed at outlet to keep the total mass conserved. Hybrid scheme for the convection and central 
difference for diffusion are used for space discretization, and fully implicit method is applied for the time discretization.

A $3 \times 3$ matrix composed of three different grids $(51 \times 68,60 \times 90,90 \times 150)$ by three time steps $(0.04 \mathrm{~s}$, $0.02 \mathrm{~s}, 0.01 \mathrm{~s})$ is used to investigate the grid convergence and time convergence. One may think that the solution should not change with different time step since the steady state equations are solved basically. However, the projection method used in this study introduces certain error which is a function of the time step.

Streamfunction contours calculated with $(90 \times 150,0.01 \mathrm{~s})$ are plotted in Figure 5.6. Two eddies are observed: one is the big recirculation eddy which determines the reattachment length, the other one is the small eddy at the corner. These match the experimental observation closely. The standard k- $\varepsilon$ turbulence model with the wall function, however, is not able to capture the small eddy (Lien and Leschziner, 1994). This means that the cubic low-Reynolds number turbulence model is more capable of resolving secondary eddies than the standard $\mathrm{k}-\varepsilon$ turbulence model.

The reattachment length calculated with different time steps and different grids are shown in Table 5.1, Figure 5.7, and Figure 5.8. The reattachment point is the location where $\mathrm{U}$ equals to zero, and it is determined by applying the cubic spline interpolation, so that the order of interpolation method is higher than the one of the numerical scheme in the solver. This helps reducing the contamination from the postprocessing method.

The extrapolated reattachment length with different extrapolation methods (Celik, et al. 2005, Celik and $\mathrm{Li}, 2005)$ are listed in Table 5.2 and 5.3. With the fine grid (90x150), the normalized reattachment value extrapolated with the solutions at different time step is at 6.17-6.22; and with the smallest time step $(\mathrm{dt}=0.01)$, the extrapolated one with different grids is 6.17 . The reattachment length by DNS (Le \& Moin, 1992) is 6.25. The relative error introduced by the model should be less than (6.25$6.17) / 6.25=1.3 \%$, which is one order of magnitude higher than the extrapolated relative error (ERE) (Celik and $\mathrm{Li}, 2005$ ) induced by the finite grids. Table 5.2 and 5.3 also shows that the ERE introduced by the finite time step is one order of magnitude lower than the ERE resulted from the finite grid size. 
The apparent order calculated with the power law (or Richardson Extrapolation) is 1.0-1.3 for the time convergence, and is 2.1-2.15 for the grid convergence. These agree well the orders of the schemes in time and in space.

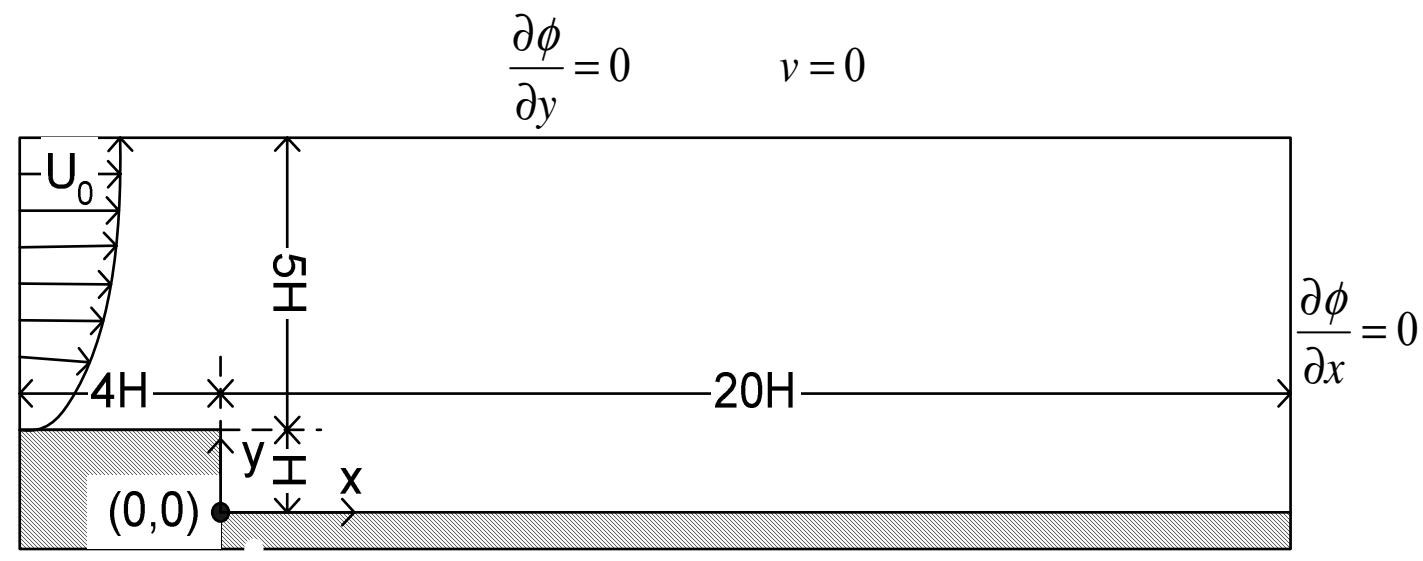

Figure 5-5 The computational domain for the backward facing step flow of (Le and Moin, 1992)

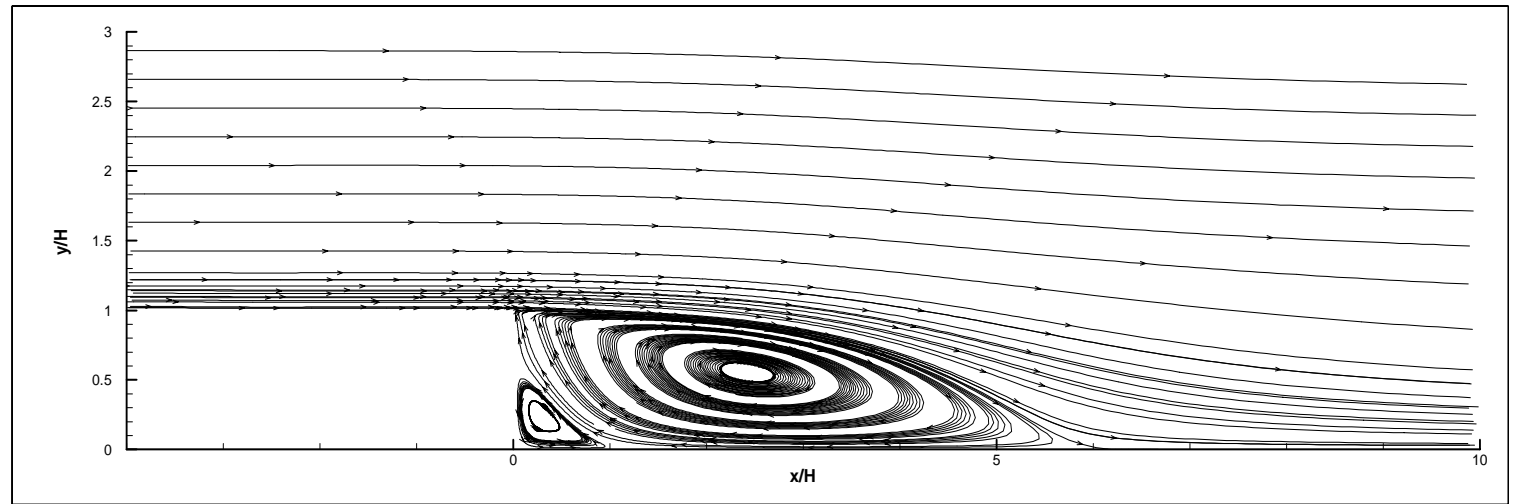

Figure 5-6 Streamfunction contours in and around recirculation zone (90x150, 0.01s)

Table 5.1 Reattachment lengths calculated with different grids and time steps

\begin{tabular}{|l|r|r|r|}
\hline grids dt & 0.04 & 0.02 & 0.01 \\
\hline $51 \times 68$ & 5.992 & 5.987 & 5.985 \\
\hline $60 \times 90$ & 6.119 & 6.111 & 6.107 \\
\hline $90 \times 150$ & 6.189 & 6.178 & 6.173 \\
\hline
\end{tabular}


Table 5.2 Extrapolated with different methods based on time step variations

\begin{tabular}{|c|l|r|r|r|}
\hline & grids & \multicolumn{1}{|c|}{$51 \times 68$} & $60 \times 90$ & $90 \times 150$ \\
\hline & p & 2.1 & 2.15 & 2.15 \\
\hline \multirow{3}{*}{$\begin{array}{c}\text { Extrapolated } \\
\text { value }\end{array}$} & power law & 5.98 & 6.10 & 6.17 \\
\cline { 2 - 5 } & cubic spline & 5.98 & 6.10 & 6.17 \\
\cline { 2 - 5 } & polynomial & 5.98 & 6.10 & 6.17 \\
\cline { 2 - 5 } ERE & AES & 5.99 & 6.11 & 6.17 \\
\hline \multirow{5}{*}{} & power law & $2.2 \mathrm{E}-04$ & $6.6 \mathrm{E}-04$ & $6.8 \mathrm{E}-04$ \\
\cline { 2 - 5 } & cubic spline & $3.3 \mathrm{E}-04$ & $6.6 \mathrm{E}-04$ & $8.1 \mathrm{E}-04$ \\
\cline { 2 - 5 } & polynomial & $2.8 \mathrm{E}-04$ & $6.6 \mathrm{E}-04$ & $7.6 \mathrm{E}-04$ \\
\cline { 2 - 5 } & AES & $3.3 \mathrm{E}-05$ & $1.6 \mathrm{E}-04$ & $9.7 \mathrm{E}-05$ \\
\hline
\end{tabular}

Table 5.3 Extrapolation with different methods based on grid variations

\begin{tabular}{|c|l|r|r|r|}
\hline & $\mathrm{dt}$ & 0.04 & 0.02 & 0.01 \\
\hline & $\mathrm{p}$ & 2.1 & 2.15 & 2.15 \\
\hline \multirow{4}{*}{$\begin{array}{c}\text { Extrapolated } \\
\text { value }\end{array}$} & power law & 6.20 & 6.19 & 6.18 \\
\cline { 2 - 5 } & cubic spline & 6.23 & 6.22 & 6.22 \\
\cline { 2 - 5 } & polynomial & 6.20 & 6.18 & 6.18 \\
\cline { 2 - 5 } & AES & 6.18 & 6.17 & 6.17 \\
\hline \multirow{4}{*}{ ERE } & power law & $1.9 \mathrm{E}-03$ & $1.7 \mathrm{E}-03$ & $1.7 \mathrm{E}-03$ \\
\cline { 2 - 5 } & cubic spline & $7.3 \mathrm{E}-03$ & $7.2 \mathrm{E}-03$ & $6.9 \mathrm{E}-03$ \\
\cline { 2 - 5 } & polynomial & $1.3 \mathrm{E}-03$ & $1.0 \mathrm{E}-03$ & $1.0 \mathrm{E}-03$ \\
\cline { 2 - 5 } & AES & $7.0 \mathrm{E}-04$ & $6.6 \mathrm{E}-04$ & $6.5 \mathrm{E}-04$ \\
\hline
\end{tabular}




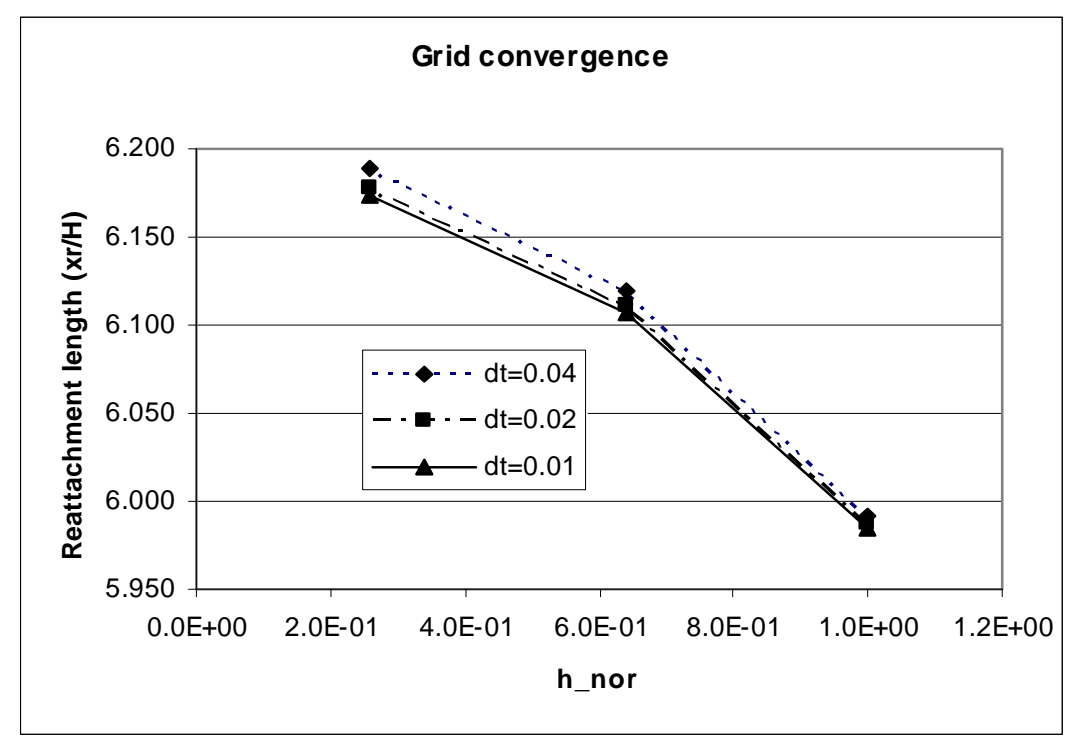

Figure 5-7 Grid convergence of the reattachment length with respect to the normalized grid size

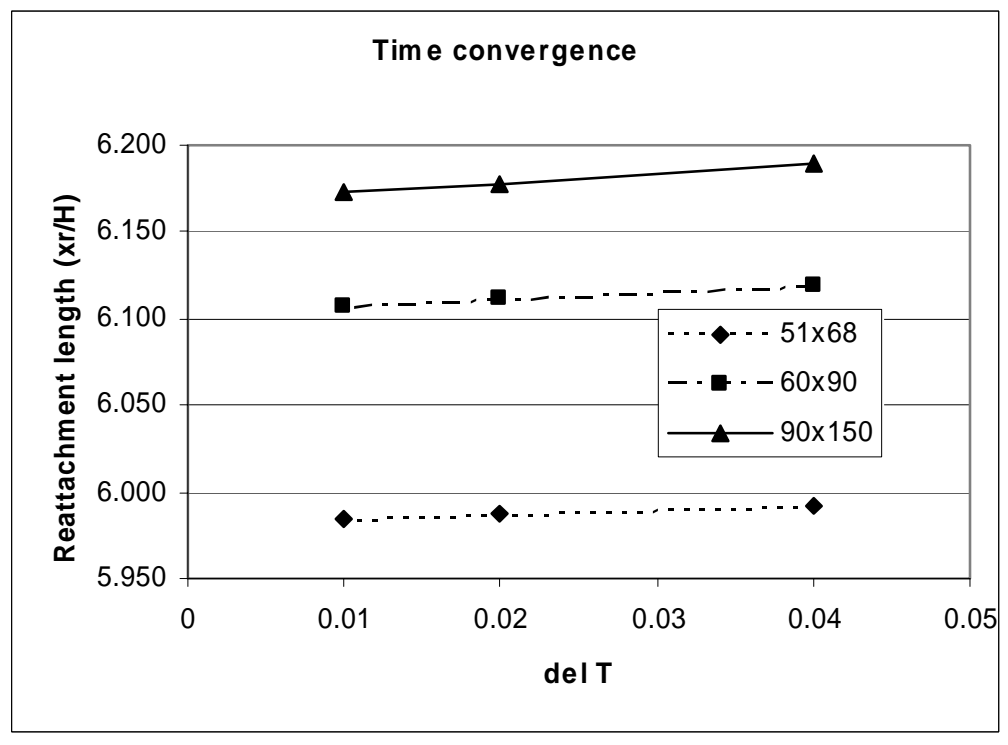

Figure 5-8 Time convergence of the reattachment length with respect to the time step

The calculated velocity profiles at different locations with the finest grid and time step are compared with the DNS data in Figure 5.9. It is seen that the inlet profiles match perfectly at $\mathrm{x} / \mathrm{H}=-3$, which is the proof that the inlet boundary condition was specified correctly. Although the velocity profiles in the 
recirculation region $(\mathrm{x} / \mathrm{H}=4)$ reveal some differences in the near wall region, the results are overall superior compared to the results with linear eddy viscosity models and comparable to the large eddy simulation and detached eddy simulation (Lien and Leschziner, 1994; Dejoan and Leschziner, 2004).

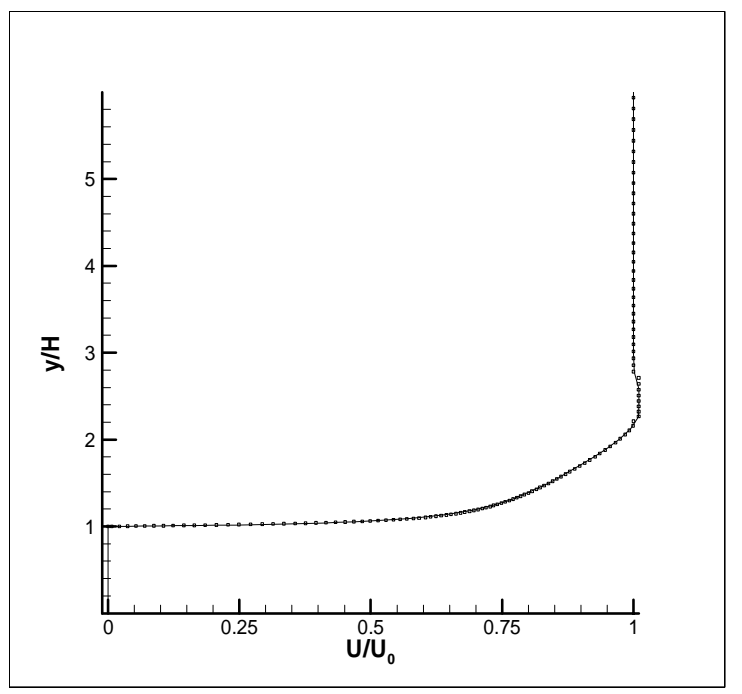

(a) $\quad \mathrm{x} / \mathrm{H}=-3$

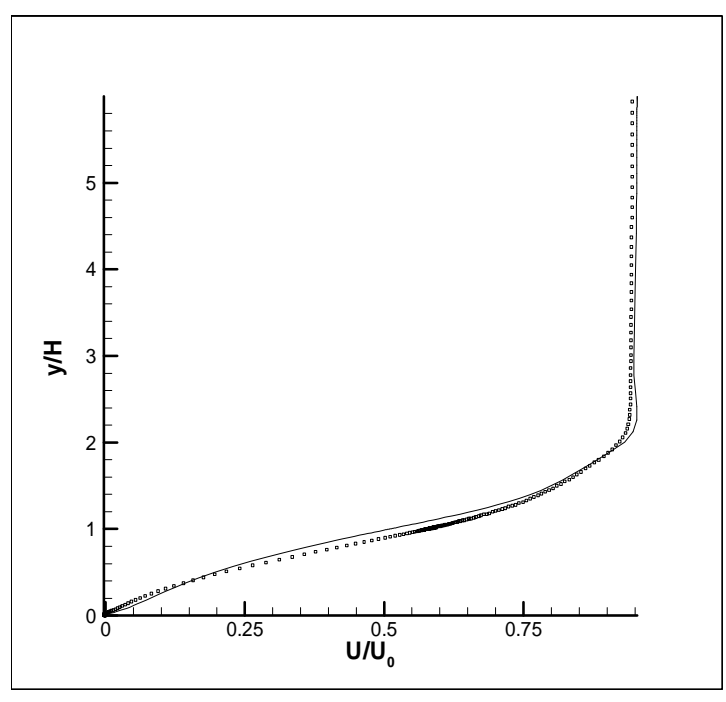

(c) $\quad \mathrm{x} / \mathrm{H}=6$

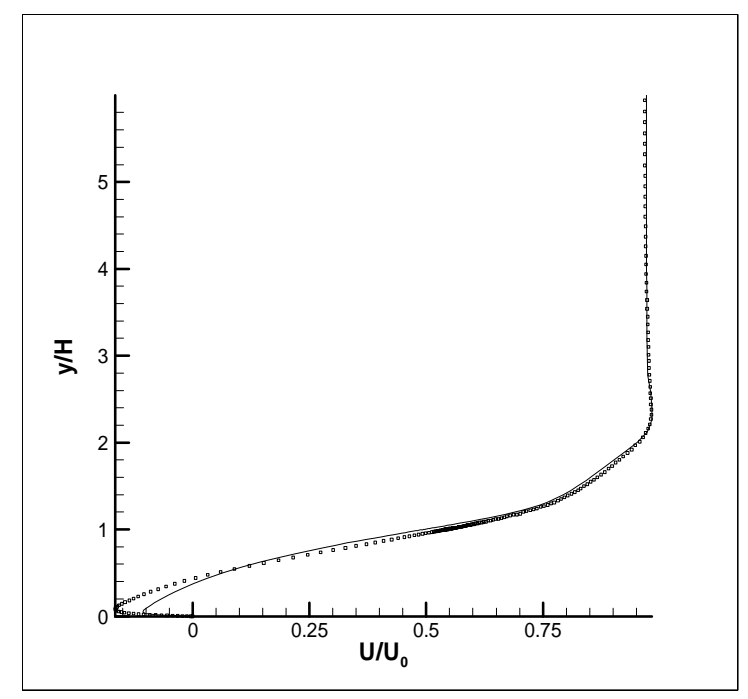

(b) $\quad \mathrm{x} / \mathrm{H}=4$

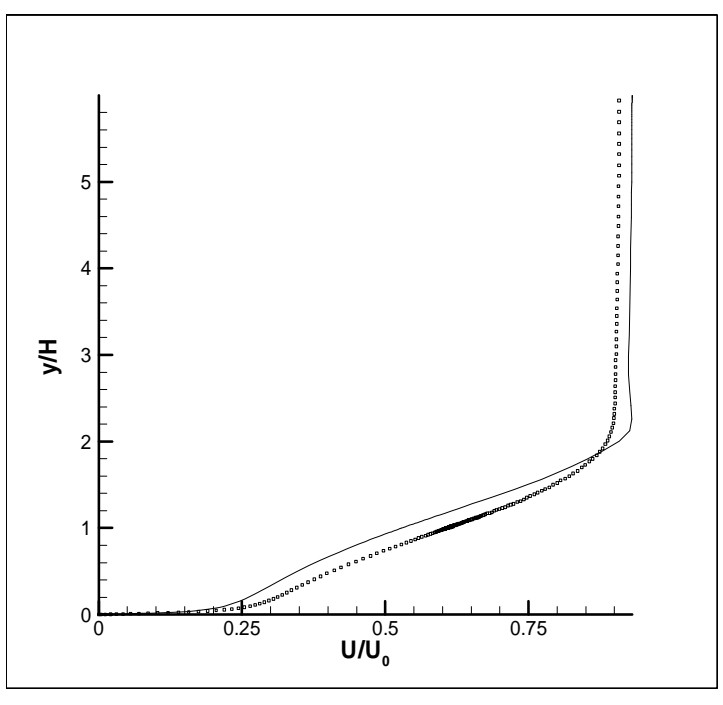

(d) $\quad \mathrm{x} / \mathrm{H}=10$

Figure 5-9 Streamwise velocity profiles (solid line: simulations with $90 \times 150$ and $\mathrm{dt}=0.01 \mathrm{~s}$; symbols: DNS by Le \& Moin)

Figure 5.10 presents the turbulent kinetic energy profiles at different locations. At the inlet, the $\mathrm{k}$ profile overlaps the DNS data, which again verifies the inlet boundary condition was imposed correctly. 
The cubic turbulence not only captures the trend but also is capable of predicting accurate turbulent kinetic energy in the whole domain. It is also noticed that the maximum of $\mathrm{k}$ at $\mathrm{x} / \mathrm{H}=4$ is twice more than the one at the inlet channel, which means a lot of turbulence is generated after the flow passes the step. The location, where $\mathrm{k}$ reaches its maximum, matches the region where the velocity dramatically changes, which furthermore confirms that the turbulence is generated mostly by the shear due to the expansion.

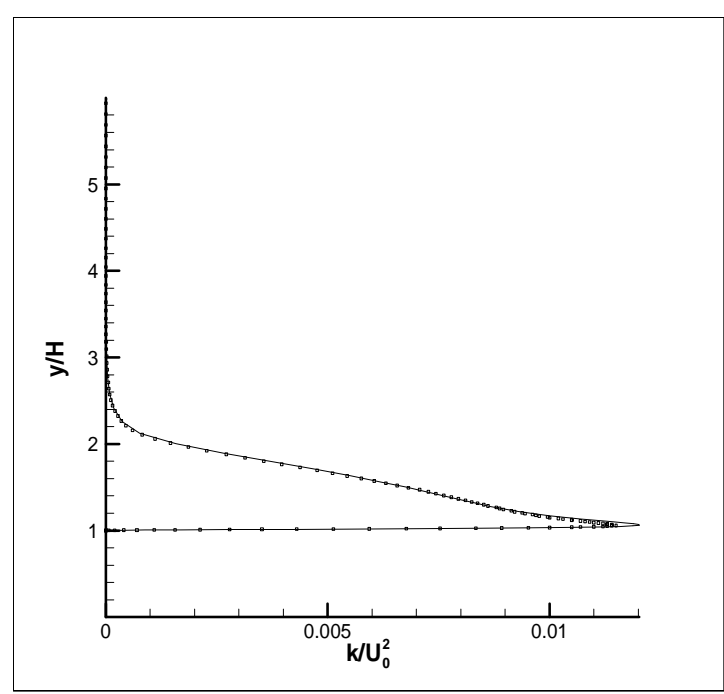

(a) $\quad \mathrm{x} / \mathrm{H}=-3$

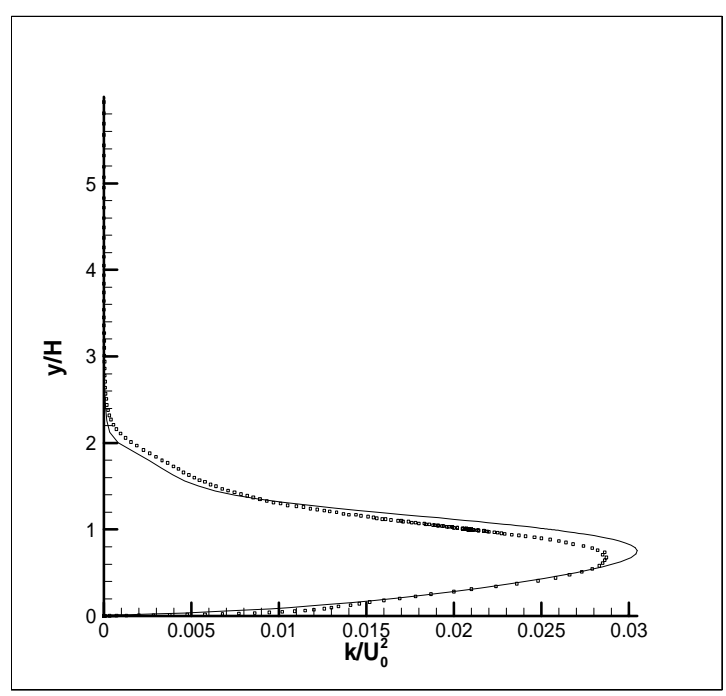

(c) $\quad \mathrm{x} / \mathrm{H}=6$

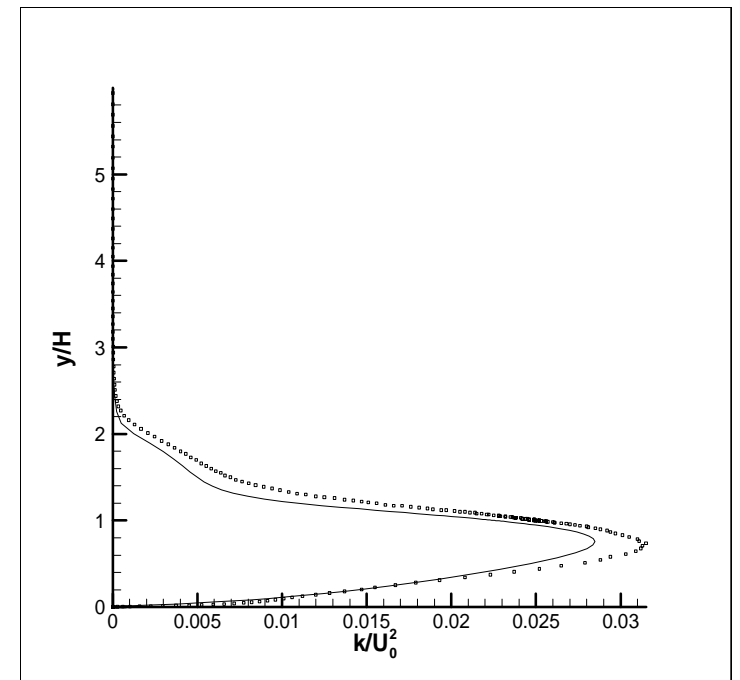

(b) $\quad \mathrm{x} / \mathrm{H}=4$

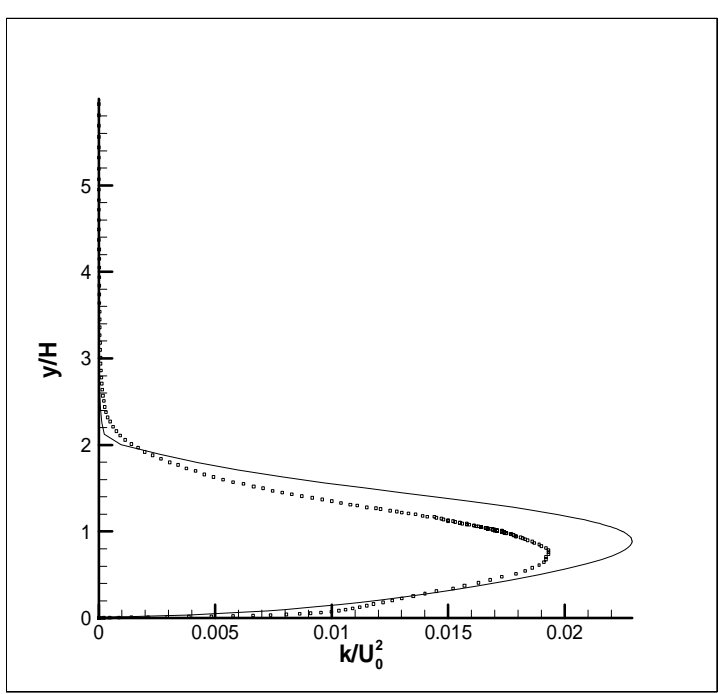

(d) $\quad \mathrm{x} / \mathrm{H}=10$

Figure 5-10 Turbulent kinetic energy profiles (solid line: simulations with 90x150 and dt=0.01s; symbols: DNS by Le \& Moin) 
The normalized $\mathrm{u}_{\mathrm{rms}}$ profiles are compared with DNS data in Figure 5.11. The urms at $\mathrm{x} / \mathrm{H}$ with the cubic model is less than the one with DNS, which is similar as the results in the turbulent channel flow. However, in the recirculation zone, this deficiency is not observed. In the recovery region, the DNS data showed the influence of the wall on the streamwise normal stresses, whereas, the cubic model didn't capture it. In general, the overall agreement is very satisfactory.

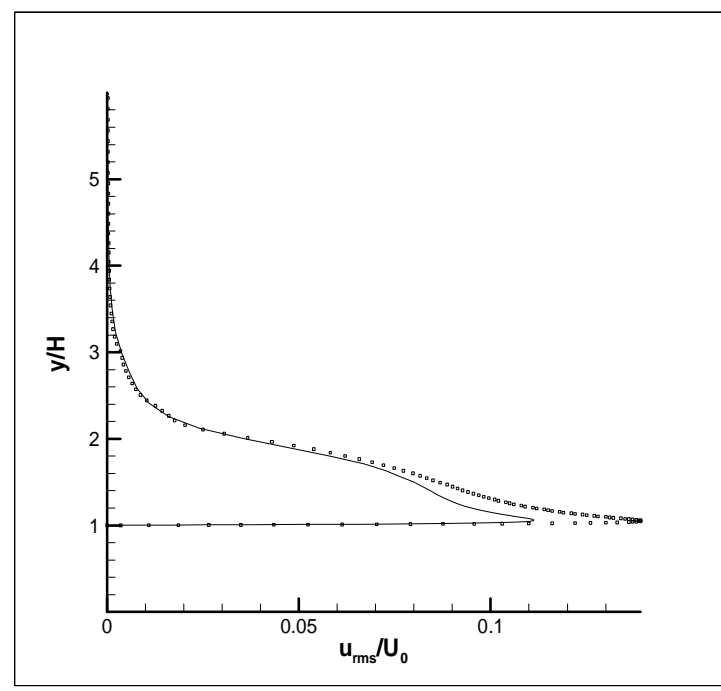

(a) $\quad \mathrm{x} / \mathrm{H}=-3$

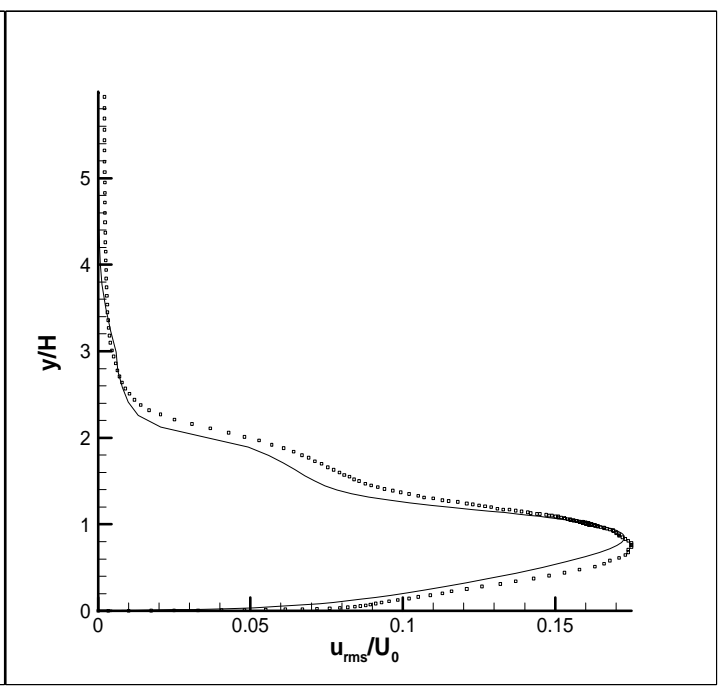

(b) $\quad \mathrm{x} / \mathrm{H}=4$
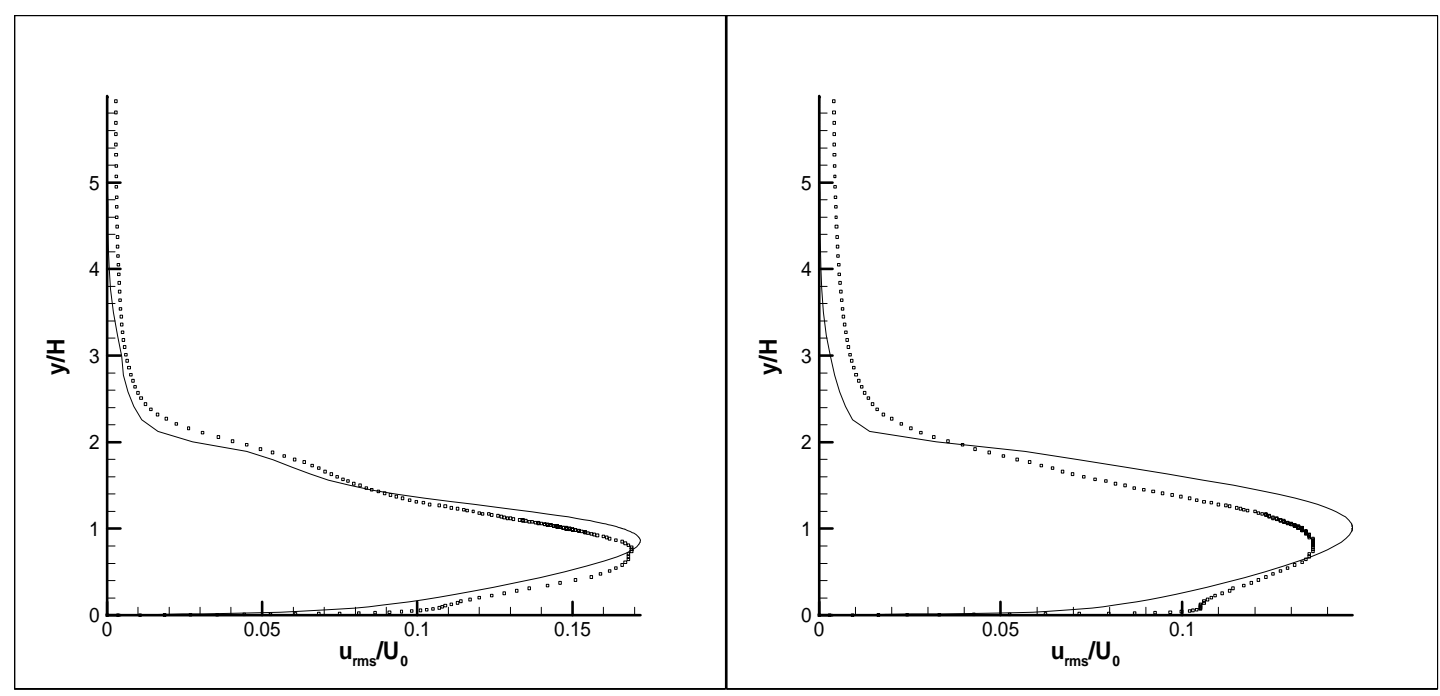

(c) $\mathrm{x} / \mathrm{H}=6$

(d) $\quad \mathrm{x} / \mathrm{H}=10$

Figure 5-11 $\mathrm{u}_{\mathrm{rms}} / \mathrm{U} \_0$ (solid line: simulations with $90 \times 150$ and $\mathrm{dt}=0.01 \mathrm{~s}$; symbols: DNS by Le \& Moin) 
The $\mathrm{v}_{\mathrm{rms}}$ are also well resolved by the cubic low-Reynolds-number turbulence model as shown in Figure 5.12. The normal stresses in the recirculation region are more isotropic than the ones at the near wall region of the inlet channel, because the pressure redistribution term is rather small in that region compared to the generation by the shear stresses.

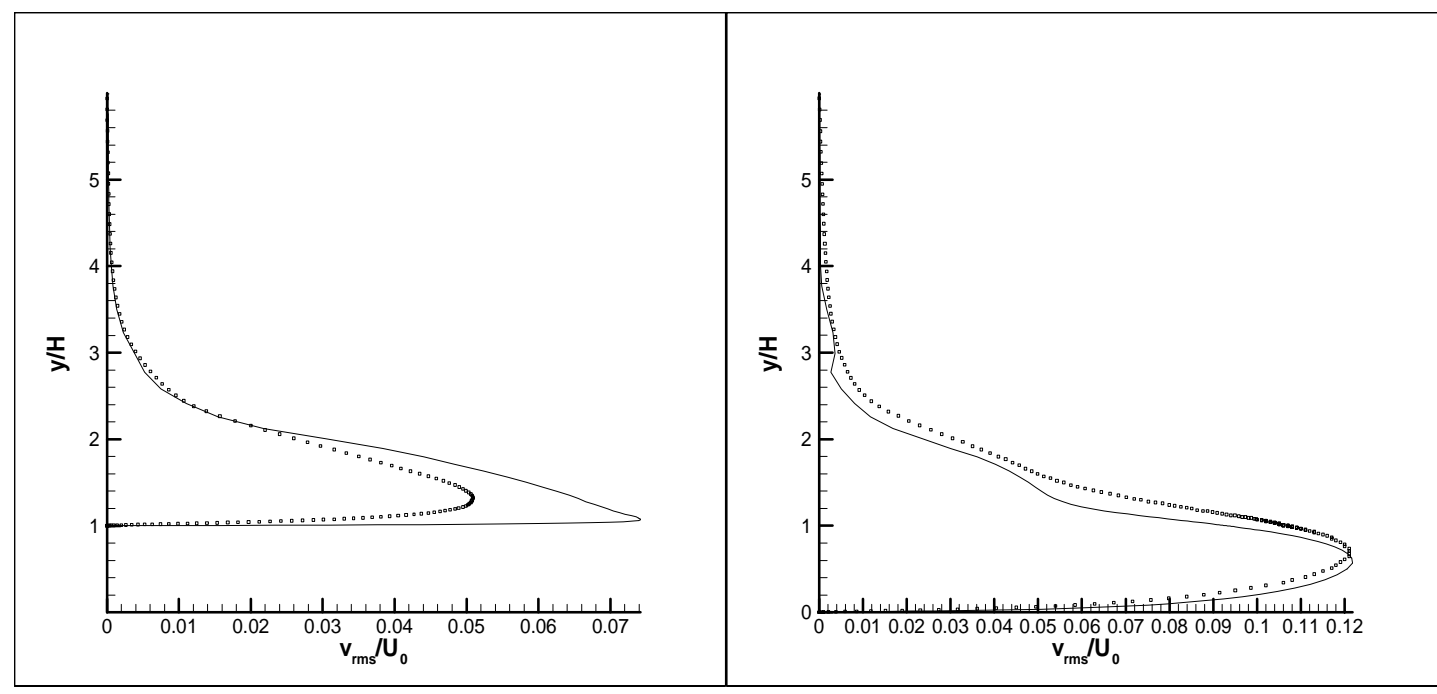

(a) $\mathrm{x} / \mathrm{H}=-3$

(b) $\quad \mathrm{x} / \mathrm{H}=4$
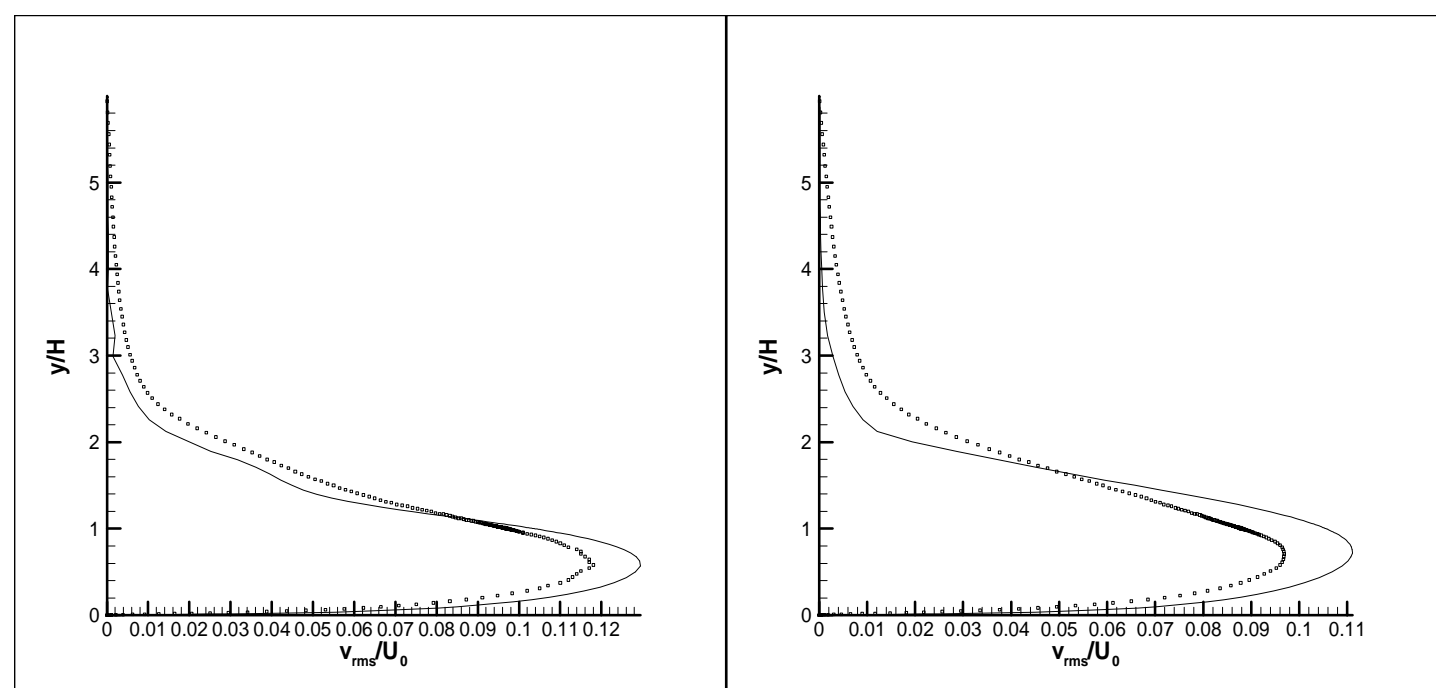

(c) $\mathrm{x} / \mathrm{H}=6$

(d) $\quad \mathrm{x} / \mathrm{H}=10$

Figure 5-12 $\mathrm{v}_{\mathrm{rms}} / \mathrm{U} \_0$ (solid line: simulations with $90 \times 150$ and $\mathrm{dt}=0.01 \mathrm{~s}$; symbols: DNS by Le \& Moin) 
Although it is a $2 \mathrm{D}$ calculation and the mean transverse velocity is assumed to be zero, the $\mathrm{w}_{\mathrm{rms}}$ calculated with Eq. 4.10 is in excellent agreement with DNS data (Figure 5.13), which implies that the variation of the mean quantities in the spanwise direction is indeed negligible.
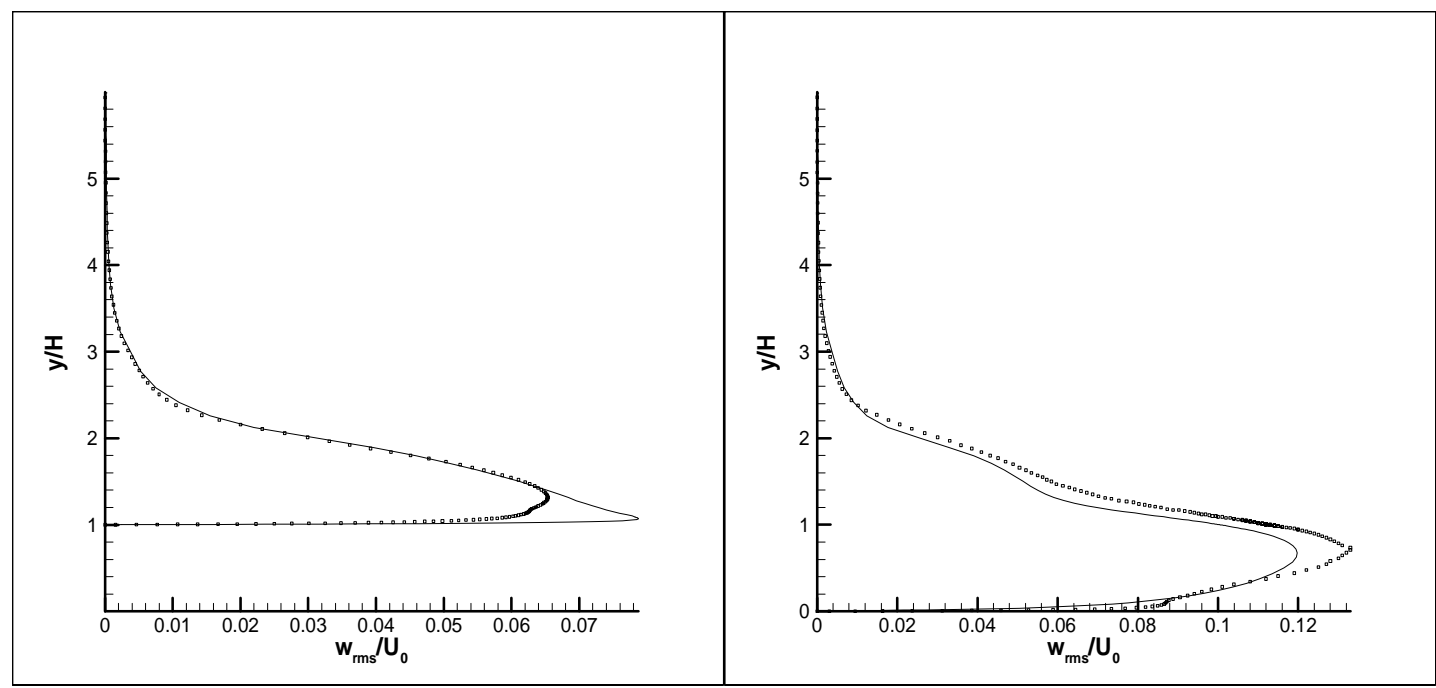

(a) $\mathrm{x} / \mathrm{H}=-3$

(b) $\quad \mathrm{x} / \mathrm{H}=4$
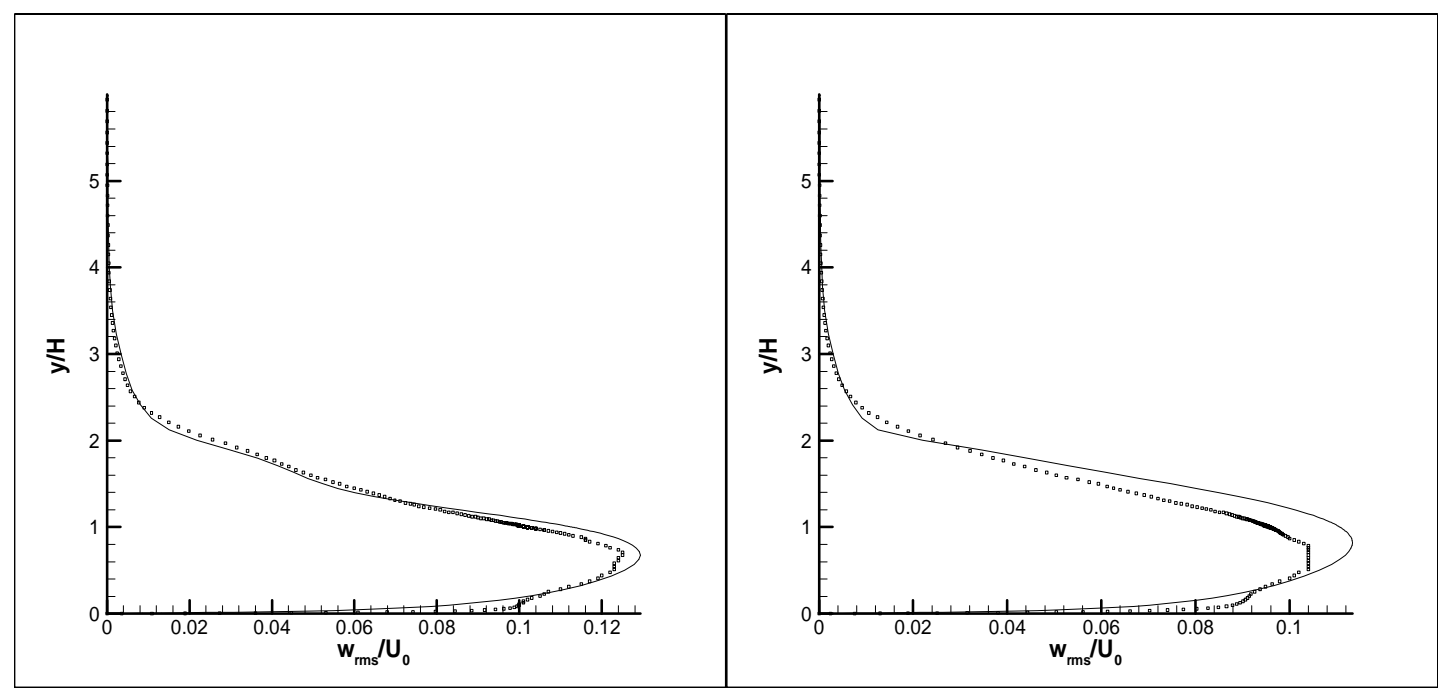

(c) $\quad \mathrm{x} / \mathrm{H}=6$

(d) $\quad \mathrm{x} / \mathrm{H}=10$

Figure 5-13 wrms/U_0 (solid line: simulations with $90 \times 150$ and $\mathrm{dt}=0.01 \mathrm{~s}$; symbols: DNS by Le \& Moin) 
Figure 5.14 shows that turbulence generation rate achieves its maximum along the shear layer in the immediate downstream of the step edge as expected.

The $\mathrm{k}$ and $\varepsilon$ contours are shown in Figure 5.15 and Figure 5.16 respectively. In channel region, the turbulent kinetic energy reaches its maximum in the region very close to wall, as was also observed from the channel flow simulation. After the step, the turbulent kinetic energy is generated along the shear layer and reach its maximum (around 0.03 ) at about $\mathrm{x} / \mathrm{H}=5.5$. Then the maximum value decreases as the velocity recovers and the dissipation takes the lead. The $\varepsilon$ contours, however, show that the maximum turbulent dissipation rate appears immediately after the step, which corresponds to the maximum turbulence generation region.

The turbulent eddy viscosity contours shown in Figure 5.17, however, achieve a maximum at far downstream rather than the place where turbulent kinetic energy reaches the maximum. This is because the dissipation rate maintains certain levels when $\mathrm{k}$ is large. Again, Figure 5.18 and 5.19 show that the turbulence in the recirculation region is approximately isotropic.

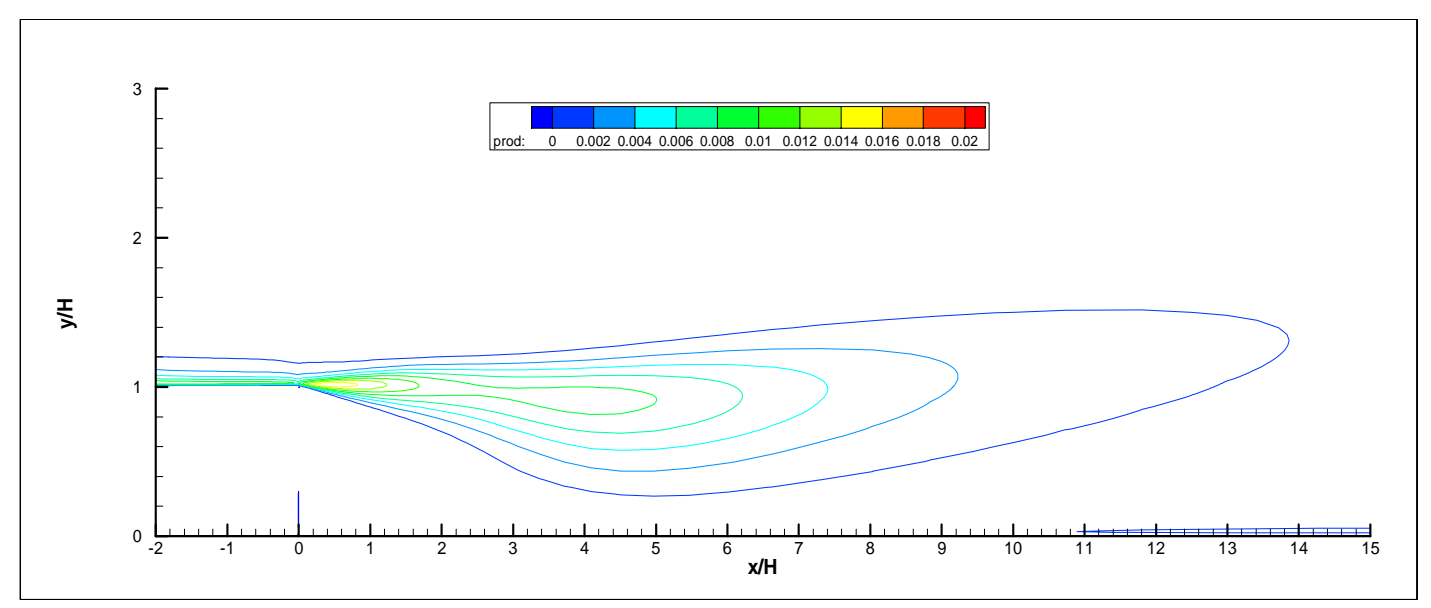

Figure 5-14 Turbulence generation rate contours 


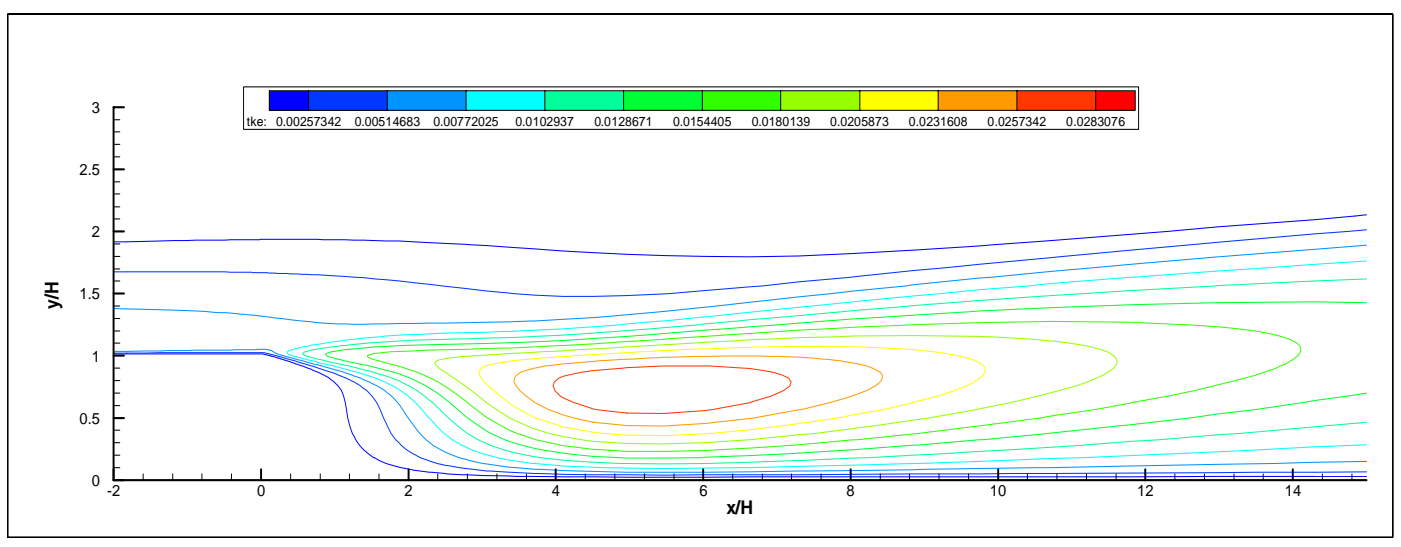

Figure 5-15 Normalized turbulent kinetic energy contours

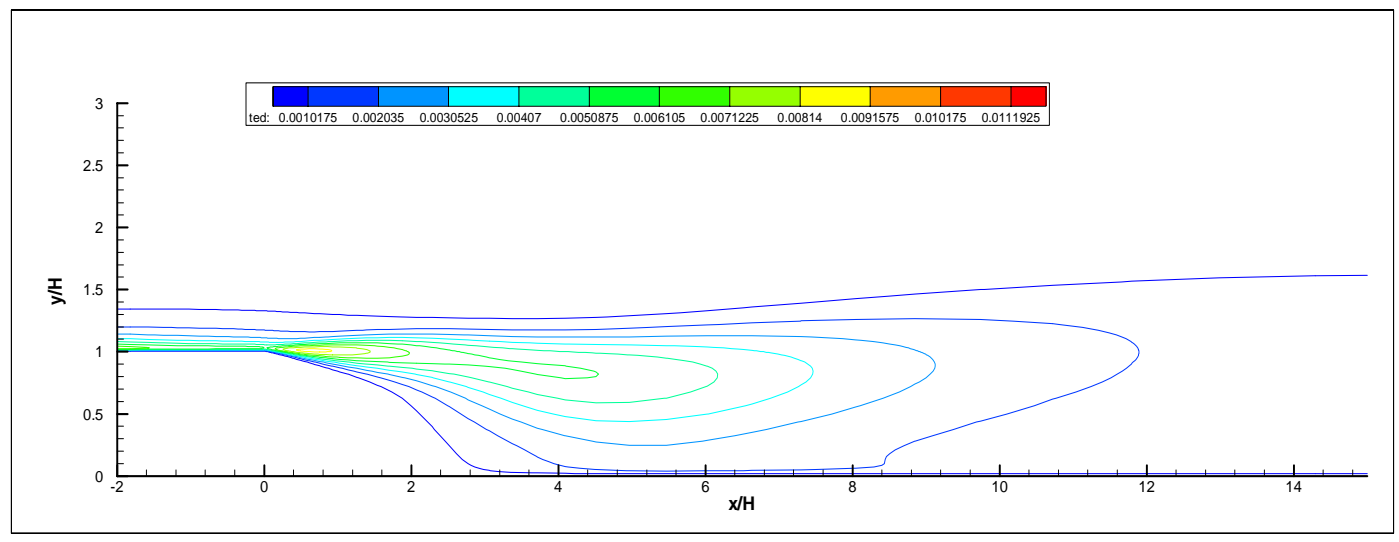

Figure 5-16 Normalized $\varepsilon$ contours

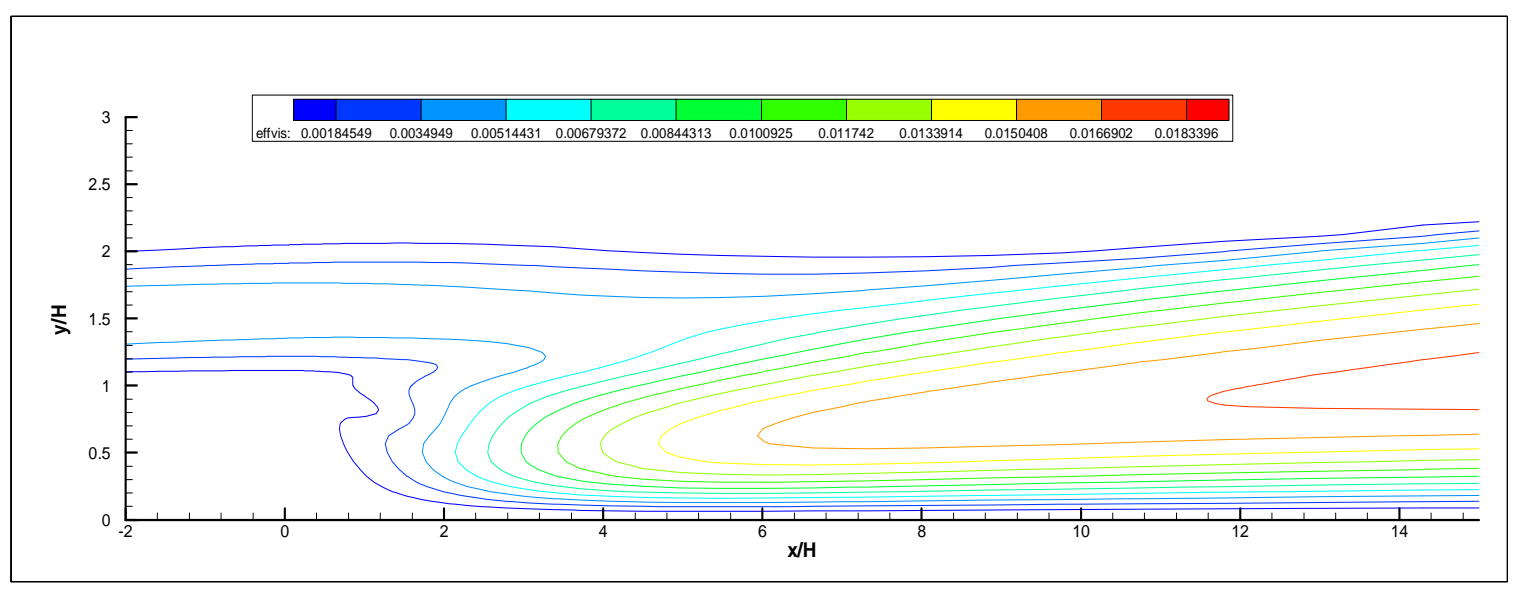

Figure 5-17 Eddy viscosity contours 


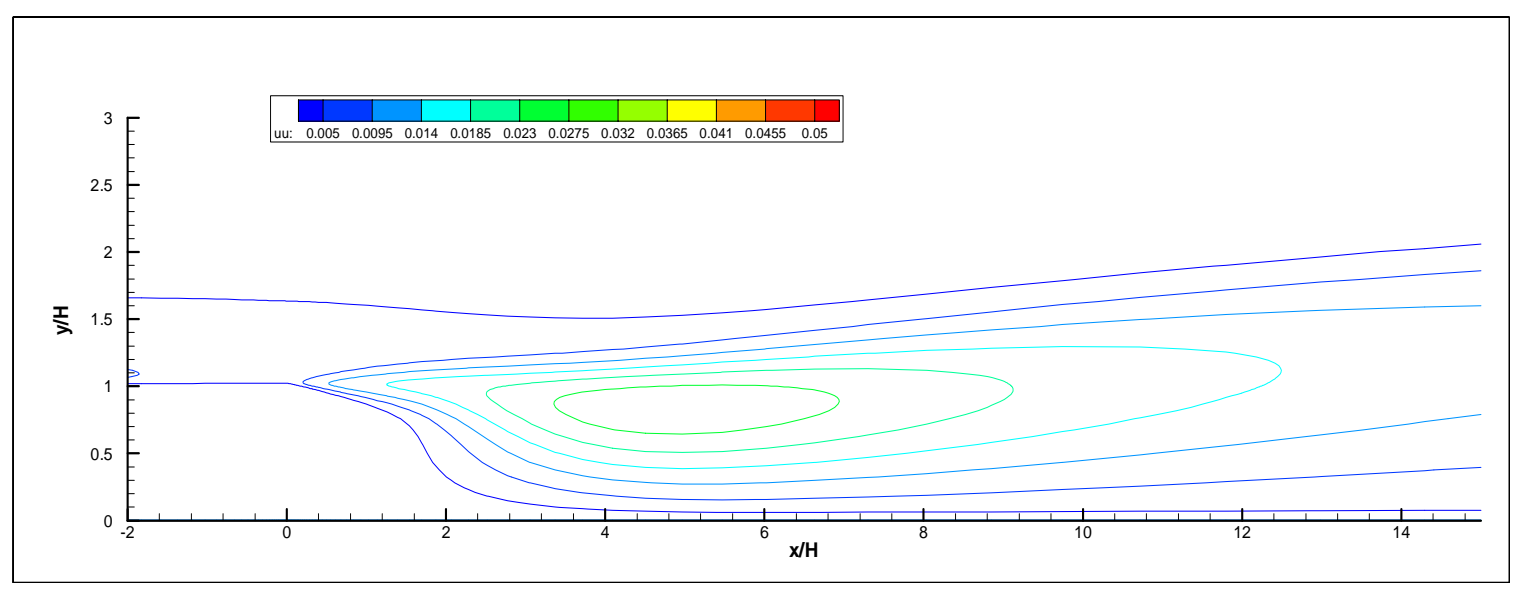

Figure 5-18 $\left(\mathrm{u}_{\mathrm{rms}}\right)^{2}$ contours

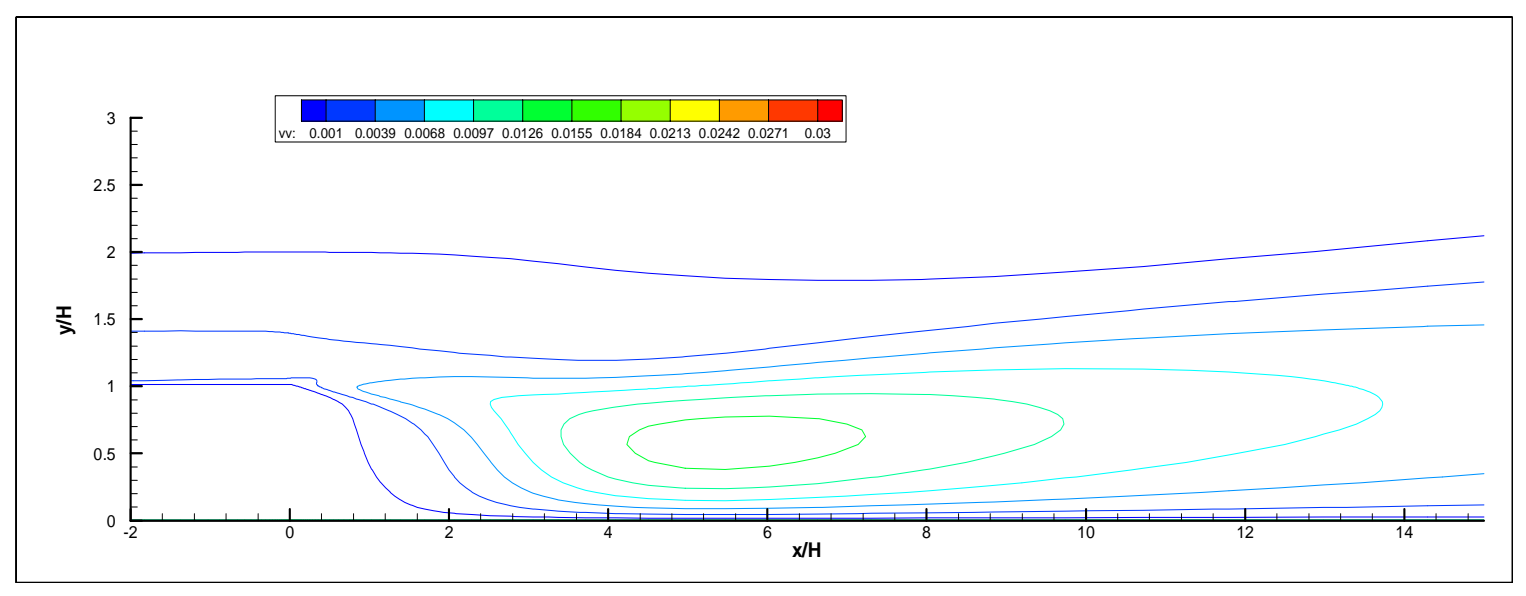

Figure 5-19 $\left(\mathrm{v}_{\mathrm{rms}}\right)^{2}$ contours

\subsection{Flow around a square cylinder}

The flow past long square cylinders exposed to uniform approaching flow is an interesting test case because the geometry is simple, but the flow is complex with unsteady separation. Alternating vortices are shed from the cylinder and transported downstream, where they retain their identity in a Karman vortex street for a considerable distance. These vortices are predominantly two-dimensional and so is the timemean flow, but large-scale three-dimensional structures exist which lead to a modulation of the shedding frequency. The approaching stagnation flow is basically invisid and thin laminar boundary layers form on 
the forward surfaces of the cylinder. The flow separates at the front edges and a flapping shear layer develops on the sides of the cylinder, which is initially laminar but becomes turbulent fairly quickly.

For the square cylinder, the detailed experiments are those reported by Lyn et al. (1995) Durao, et al (1988) and Lyn and Rodi (1994). The situation investigated by them $(\mathrm{Re}=22,000)$ has become a standard benchmark case for this flow. In the literature, the RANS simulations obtained with different turbulence models varying from the algebraic Balsdwin-Lomax model to a Reynolds-stress model (RSM) and also an LES have been reviewed by Rodi (2001); this LES suffered from a small calculation domain of only 2 cylinder widths in the spanwise direction. The flow was then used as a test case for an LES workshop in 1995 in Rottach-Egern, Germany, and 16 different calculations submitted by 9 groups are reported in Rodi et al. (1997). These calculations were all performed on a computational domain of 4D in the spanwise direction, extending 4.5D upstream of the cylinder, $6.5 \mathrm{D}$ on either side of the cylinder and at least $14.5 \mathrm{D}$ downstream of the cylinder. As will be shown shortly, there was a great variance in the results, and hence the same test case was posed again for a workshop at Grenoble, France, in 1996. The same calculation domain was prescribed as in the earlier workshop. 20 calculations from 7 groups are summarized by Voke et al. (1997). Here, part of these results will be presented and compared to the ones obtained with the current cubic turbulence model.

In the current study, the same domain as the earlier workshops is used (Figure 5.20). Three sets of grids which are $91 \times 91,130 \times 130$ and $185 \times 185$ are used. The grids are nonuniform and the maximum $\mathrm{y}^{+}=$ 7.8, 4.8 and 1.9, respectively, for these three grids. The $185 \times 185$ grid is shown in Figure 5.21. The results with three different time steps $(0.04 \mathrm{~s}, 0.02 \mathrm{~s} 0.01 \mathrm{~s})$ are also compared as shown in Figure 5.22. The $\mathrm{u}_{\mathrm{rms}}$ variation with different time steps is negligible. The rest study has been done with the time step of $0.02 \mathrm{~s}$. The finite volume methods employ a staggered variable arrangement. Hybrid scheme is used for the convection term and central difference is used for the diffusion term. Fully implicit first order Euler scheme is used for the time discretization. 


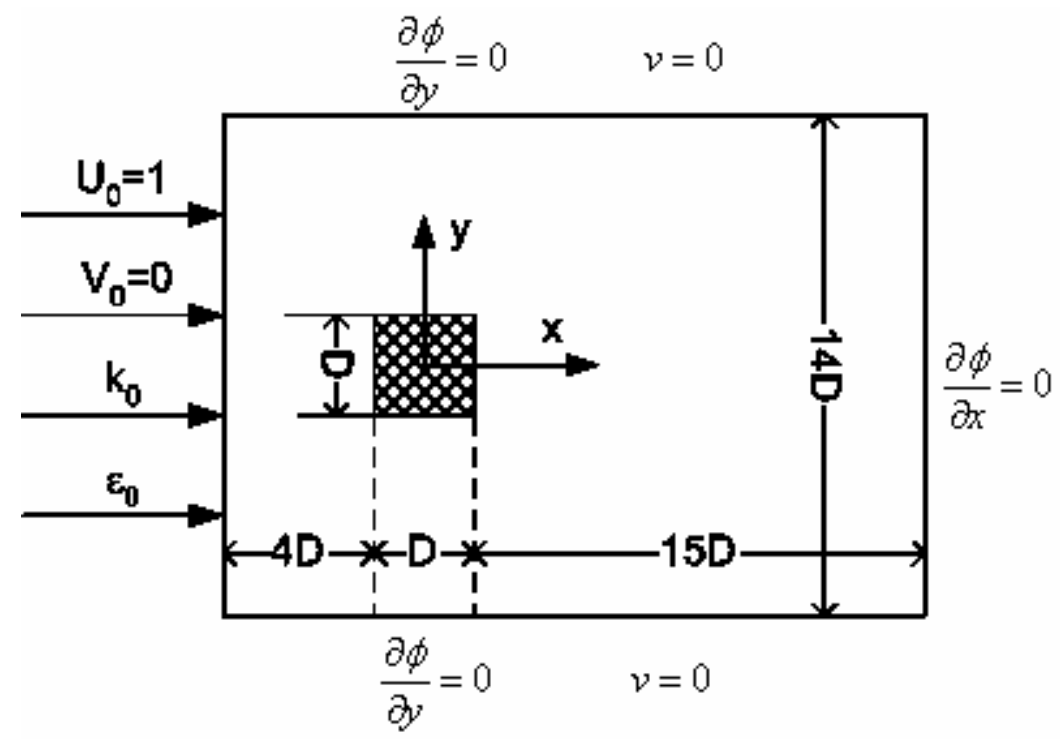

Figure 5-20 Schematic view of the computational domain for the square cylinder flow case

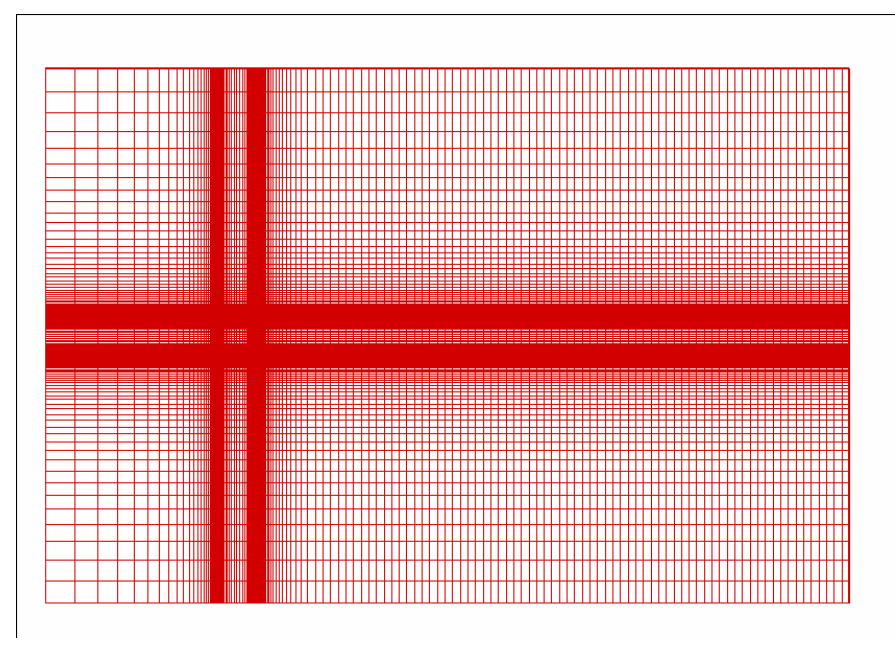

Figure $5-21$ Grid of $185 \times 185$ used for this case 


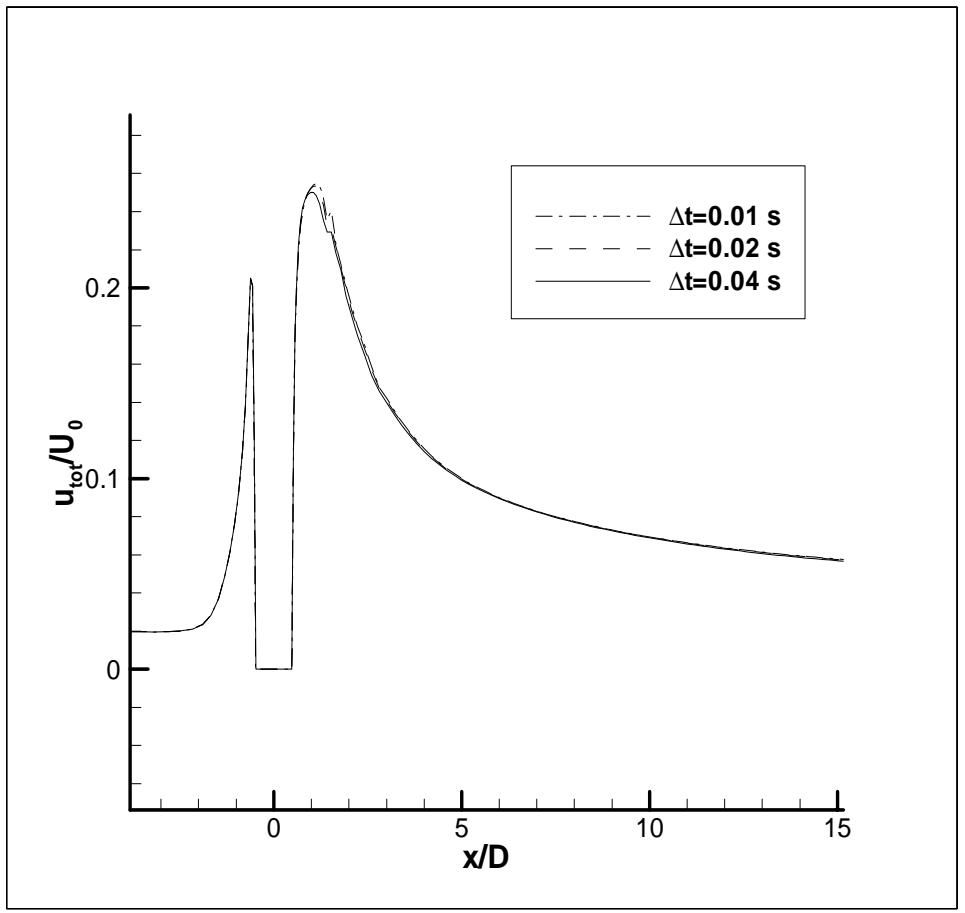

Figure 5-22 Total $u_{r m s}$ calculated with three different time steps

Figure 5.23 shows the calculated drag coefficient signal. A clear shedding frequency can be determined. The low frequency variations observed are believed to be due to three-dimensional flow structures. In the two-dimensional RANS calculations these effects cannot be simulated and hence the shedding behavior is generally regular. 


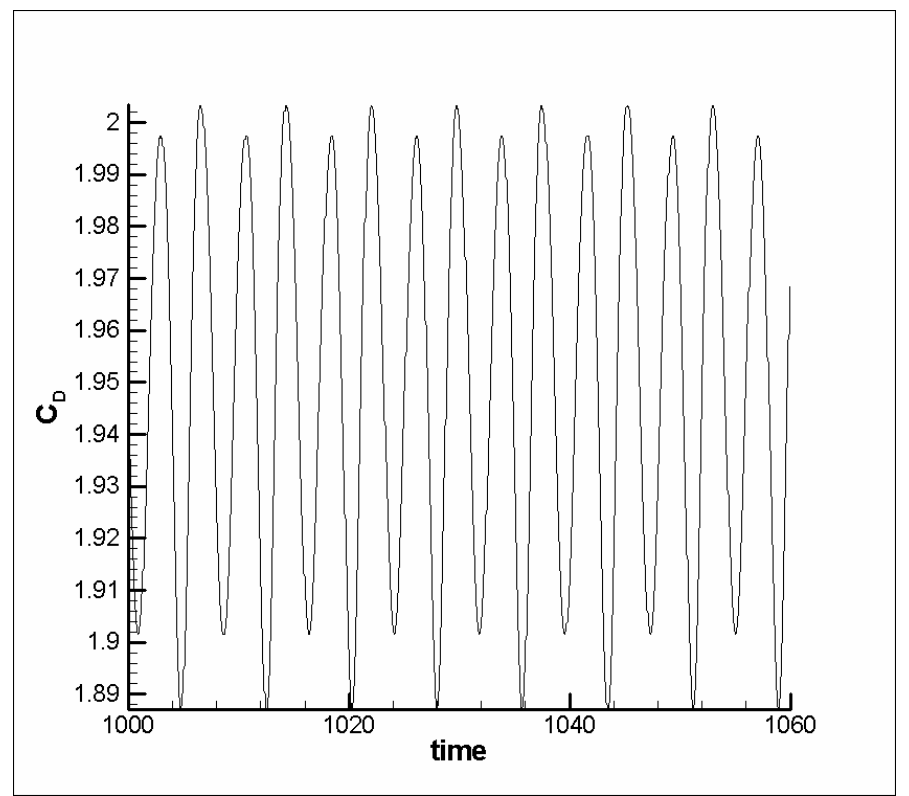

Figure 5-23 Time variation of the drag coefficient with $185 \times 185$, cubic model

Table 5.4 summarizes various global parameters such as the dimensionless shedding frequency (Strouhal number), the time-mean drag coefficient $C_{D}$, the rms values of the fluctuations of drag and lift coefficients $C_{D}$ ' and $C_{L}$ ', respectively, and the reattachment length $L_{R}$ indictaing the length of the timemean separation region behind the cylinder. Most LES calculations yielded the correct value of $\mathrm{St}=0.13$, and it appears that St is not very sensitive to the parameters of the simulation; there are, however, a few deviations from this value, notably the calculations without a subgrid-scale model yielded a higher value. Concerning the mean drag coefficient, it seems that the LES calculations using wall functions are generally close to the experimental range while those using no-slip conditions tend to produce too high values of $C_{D}$. There is also considerable variation in the recirculation length. The fluctuations of the force coefficients also show fairly large variation, where no experimental results are available for comparison.

Table 5.4 also includes results obtained with RANS models, namely by Bosch and Rodi (1998) with the standard k- $\varepsilon$ model and with a modification due to Kato and Launder (1993) (KL), and by Franke and Rodi (1993) with the Reynolds-stress model of Launder et al. (1975). In each case, either wall function (WF) or a two-layer approach applying a one-equation model in the near-wall region was used. The Strouhal number is predicted well also by most of the RANS models, but KL modification tends to produce 
somewhat too large values and the two-layer RSM an excessive value. The mean drag coefficient is significantly underpredicted by the standard k- $\varepsilon$ model, but roughly the correct value was obtained when the KL modification was used with the two-layer approach. The RSM gives the correct value of $C_{D}$ with wall functions but overpredicts it in the two-layer approach. This result is consistent with the overprediction of the length of the recirculation region by the standard k- $\varepsilon$ model and its under-prediction by the two-layer RSM.

Table 5.5 shows the present results calculated with the cubic low-Reynolds-number model for the present study as implemented in the DREAM code. As the grid is refined from $91 \times 91$ to $185 \times 185$, the St and $\mathrm{CD}$ shows little variation. This indicates that these quantities are not sensitive to the mesh size. The other quantities such as LR, however, have more variation. Unlike other RANS models, the cubic model produces reasonable values for all three quantities: $\mathrm{St}, \mathrm{C}_{\mathrm{D}}$ and $\mathrm{L}_{\mathrm{R}}$, which are as good as the LES results.

Figure 5.24 shows an example of the instantaneous vorticity contours. It is clear that the vortices are stretched a lot, which is different from the experimental observations and LES simulations, but normal for RANS calculations (Bosch and Rodi, 1998). The centers of the separated vortices stay on their side with respect to the cylinder centerline, which is also common in RANS simulations, whereas, in the flow visualization of Bearman and Trueman (1971), the centers of the vortices cross over into the other halfplane. 
Table 5.4 Global parameter for flow past square cylinder (UW=upwinding, $C D=$ central differencing, WF=wall function, NS=no slip, (1)=adjusted for different blockage, (2)=outermost mesh with embedded meshes) (Rodi, 2001)

\begin{tabular}{|c|c|c|c|c|c|c|c|c|}
\hline \multicolumn{3}{|c|}{ Calculation method } & \multirow{2}{*}{$\begin{array}{l}S t \\
0.15\end{array}$} & \multirow{2}{*}{$\begin{array}{c}C_{D} \\
2.58\end{array}$} & \multirow{2}{*}{$\frac{C_{D}^{\prime}}{0.27}$} & \multirow{2}{*}{$\begin{array}{c}C_{L}^{\prime} \\
1.33\end{array}$} & \multirow{2}{*}{$\begin{array}{c}L_{R} / D \\
1.68\end{array}$} & \multirow{2}{*}{$\frac{\stackrel{\operatorname{grid}}{N_{x} \times N_{y} \times N_{z}}}{125 \times 78 \times 20}$} \\
\hline LES & $\begin{array}{l}\text { KAWAMU, } \\
\text { No SGS, NS }\end{array}$ & 3rd UW & & & & & & \\
\hline $\begin{array}{l}\text { Rottach- } \\
\text { Egern }\end{array}$ & $\begin{array}{l}\text { UMIST2, } \\
\text { Dyn, WF }\end{array}$ & $\mathrm{CD}$ & 0.09 & 2.02 & - & - & 1.21 & $140 \times 81 \times 13$ \\
\hline \multirow[t]{4}{*}{$\begin{array}{l}\text { Rodi et al. } \\
(1997)\end{array}$} & $\begin{array}{l}\text { UKAHY2, } \\
\text { Smag, WF }\end{array}$ & $\mathrm{CD}$ & 0.13 & 2.30 & 0.14 & 1.15 & 1.46 & $146 \times 146 \times 20$ \\
\hline & $\begin{array}{l}\text { TAMU2, Dyn, } \\
\text { NS/WF }\end{array}$ & 3rd UW & 0.14 & 2.77 & 0.19 & 1.79 & 0.94 & $165 \times 113 \times 17$ \\
\hline & $\begin{array}{l}\text { UK1, Smag, } \\
\text { WF }\end{array}$ & $\mathrm{CD}$ & 0.13 & 2.20 & 0.14 & 1.01 & 1.32 & $109 \times 105 \times 20$ \\
\hline & $\begin{array}{l}\text { UK3, Smag, } \\
\text { NS }\end{array}$ & $\mathrm{CD}$ & 0.13 & 2.23 & 0.13 & 1.02 & 1.44 & $146 \times 146 \times 20$ \\
\hline LES & $\begin{array}{l}\text { NT7, LDM, } \\
\text { WF }\end{array}$ & $\mathrm{CD}$ & 0.131 & 2.05 & 0.12 & 1.39 & 1.39 & $140 \times 103 \times 32$ \\
\hline Grenoble & $\begin{array}{l}\text { UOI, Dyn.,' } \\
\text { NS }\end{array}$ & 5 th UW & 0.13 & $2.03^{(1)}$ & 0.18 & 1.29 & 1.20 & $192 \times 160 \times 48$ \\
\hline Voke & $\begin{array}{l}\text { IS3, Dyn. mix, } \\
\text { NS }\end{array}$ & 5 th UW & 0.133 & 2.79 & 0.36 & 1.68 & 1.36 & $112 \times 104 \times 32$ \\
\hline \multirow[t]{2}{*}{ (1997) } & $\begin{array}{l}\text { TIT, Dyn, } \\
\text { NS }\end{array}$ & 3rd UW & 0.131 & 2.62 & 0.23 & 1.39 & 1.23 & $121 \times 113 \times 127^{(2)}$ \\
\hline & $\begin{array}{l}\text { ST5, Smag, } \\
\text { NS }\end{array}$ & $\begin{array}{l}\text { variable } \\
\text { 3rd UW }\end{array}$ & 0.161 & 2.78 & 0.28 & 1.38 & 1.02 & $107 \times 103 \times 20$ \\
\hline $\begin{array}{l}\text { LES } \\
\text { Sohankar }\end{array}$ & $\begin{array}{l}\text { Smag, NS } \\
\text { Dyn. NS }\end{array}$ & CD & $\begin{array}{l}0.126 \\
0.125\end{array}$ & $\begin{array}{l}2.21 \\
2.04\end{array}$ & $\begin{array}{l}0.16 \\
0.20\end{array}$ & $\begin{array}{l}1.47 \\
1.22\end{array}$ & $\approx 10$ & $5 \times 25$ \\
\hline$(1998)$ & OEDSM, NS & & 0.129 & $\begin{array}{l}2.04 \\
2.25\end{array}$ & 0.20 & $\begin{array}{l}1.22 \\
1.50\end{array}$ & $\approx 1.0$ & $180 \times$ \\
\hline \multirow[t]{2}{*}{$\begin{array}{l}\text { RANS } \\
\text { Bosch } \\
(1995)\end{array}$} & $\begin{array}{l}\text { Std. } k-\varepsilon, \mathrm{WF} \\
\text { Kato-Launder } \\
k-\varepsilon, \mathrm{WF}\end{array}$ & & $\begin{array}{l}0.134 \\
0.142\end{array}$ & $\begin{array}{l}1.64 \\
1.79\end{array}$ & $\begin{array}{l}\approx 0 \\
0.012\end{array}$ & $\begin{array}{l}0.305 \\
0.614\end{array}$ & $\begin{array}{l}2.8 \\
2.04\end{array}$ & $\begin{array}{l}100 \times 76 \\
100 \times 76\end{array}$ \\
\hline & $\begin{array}{l}\text { Two-layer } k-\varepsilon \\
\text { Two-layer } \\
\text { Kato-Launder } \\
k-\varepsilon\end{array}$ & & $\begin{array}{l}0.137 \\
0.143\end{array}$ & $\begin{array}{l}1.72 \\
2.0\end{array}$ & $\begin{array}{l}\approx 0 \\
0.07\end{array}$ & $\begin{array}{l}0.426 \\
1.17\end{array}$ & $\begin{array}{l}2.4 \\
1.25\end{array}$ & $\begin{array}{l}170 \times 170 \\
170 \times 170\end{array}$ \\
\hline $\begin{array}{l}\text { RANS } \\
\text { Franke and } \\
\text { Rodi (1993) }\end{array}$ & $\begin{array}{l}\text { RMS, WF } \\
\text { Two-layer RSM }\end{array}$ & & $\begin{array}{l}0.136 \\
0.159\end{array}$ & $\begin{array}{l}2.15 \\
2.43\end{array}$ & $\begin{array}{l}0.27 \\
0.06\end{array}$ & $\begin{array}{l}1.49 \\
1.3\end{array}$ & $\begin{array}{l}0.98 \\
1.0\end{array}$ & $\begin{array}{l}70 \times 64 \\
186 \times 156\end{array}$ \\
\hline Experiments & & & 0.132 & $\begin{array}{l}1.9 \text { to } \\
2.2\end{array}$ & & & 1.38 & \\
\hline
\end{tabular}


Table 5.5 Characteristic parameters calculated with the cubic model

\begin{tabular}{ccccccc}
\hline & $\mathrm{St}$ & $\mathrm{C}_{\mathrm{D}}$ & $\mathrm{C}_{\mathrm{D}}{ }^{\prime}$ & $\mathrm{C}_{\mathrm{L}^{\prime}}$ & $\mathrm{L}_{\mathrm{R}} / \mathrm{D}$ & Grid \\
\hline \multirow{2}{*}{$\begin{array}{c}\text { RANS with Cubic } \\
\text { model }\end{array}$} & 0.126 & 1.98 & 0.0343 & 0.99 & 1.38 & $91 \times 91$ \\
\cline { 2 - 7 } & 0.135 & 1.94 & 0.025 & 0.86 & 1.82 & $135 \times 135$ \\
\cline { 2 - 7 } & 0.130 & 1.95 & 0.037 & 0.94 & 1.55 & $180 \times 180$ \\
\hline
\end{tabular}

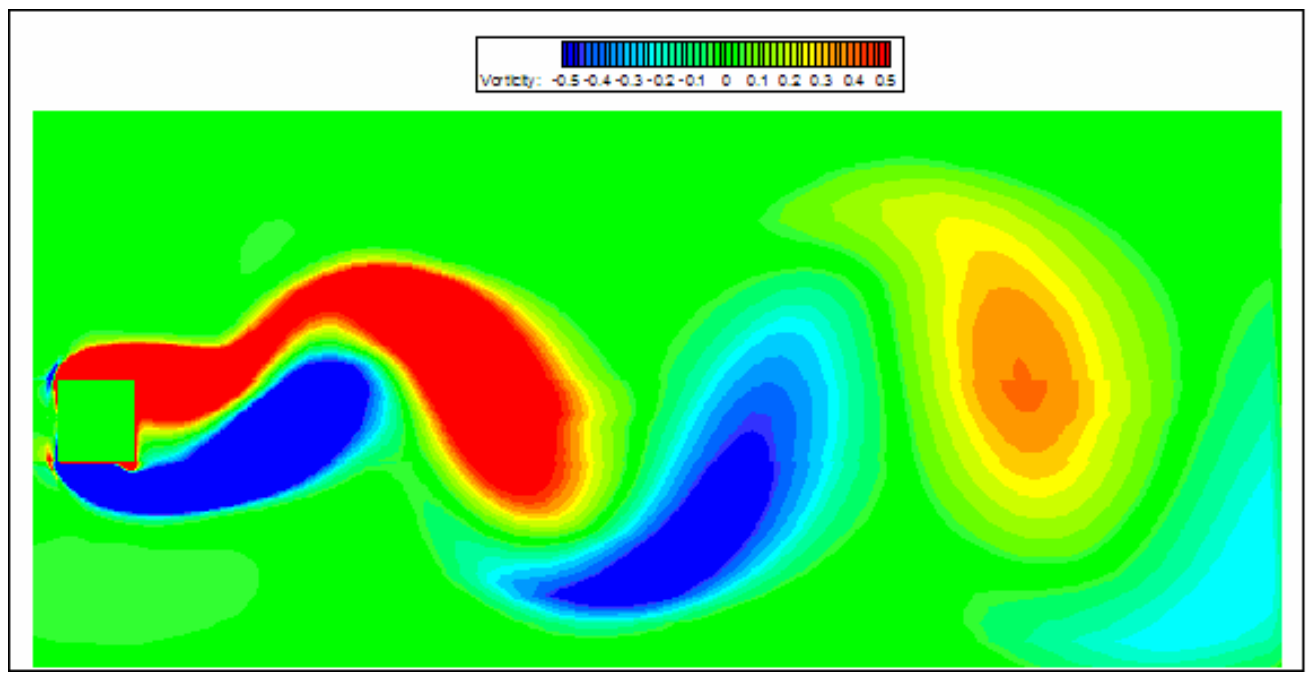

Figure 5-24 Example of the spanwise vorticity contours at one time instant

The mean streamwise velocity field is shown in Figure 5.25. It is seen that the flow is symmetric along the centerline. The flow separates from the edges of the front face of the square cylinder and bubbles are formed on both side of the cylinder, which agrees well with the experimental observation (Lyn et al. 1995). The mean total $u_{\mathrm{rms}}, v_{\mathrm{rms}}$ and turbulent kinetic energy contours are shown in Figure 5.26, Figure 5.27 and Figure 5.28. The maximum $u_{\mathrm{rms}}$ is formed along the trailing edges of the square cylinder, while the maximum $v_{\mathrm{rms}}$ is along the center-line. The total turbulent kinetic energy achieves its maximum in the downstream of the two back-facing edges. 


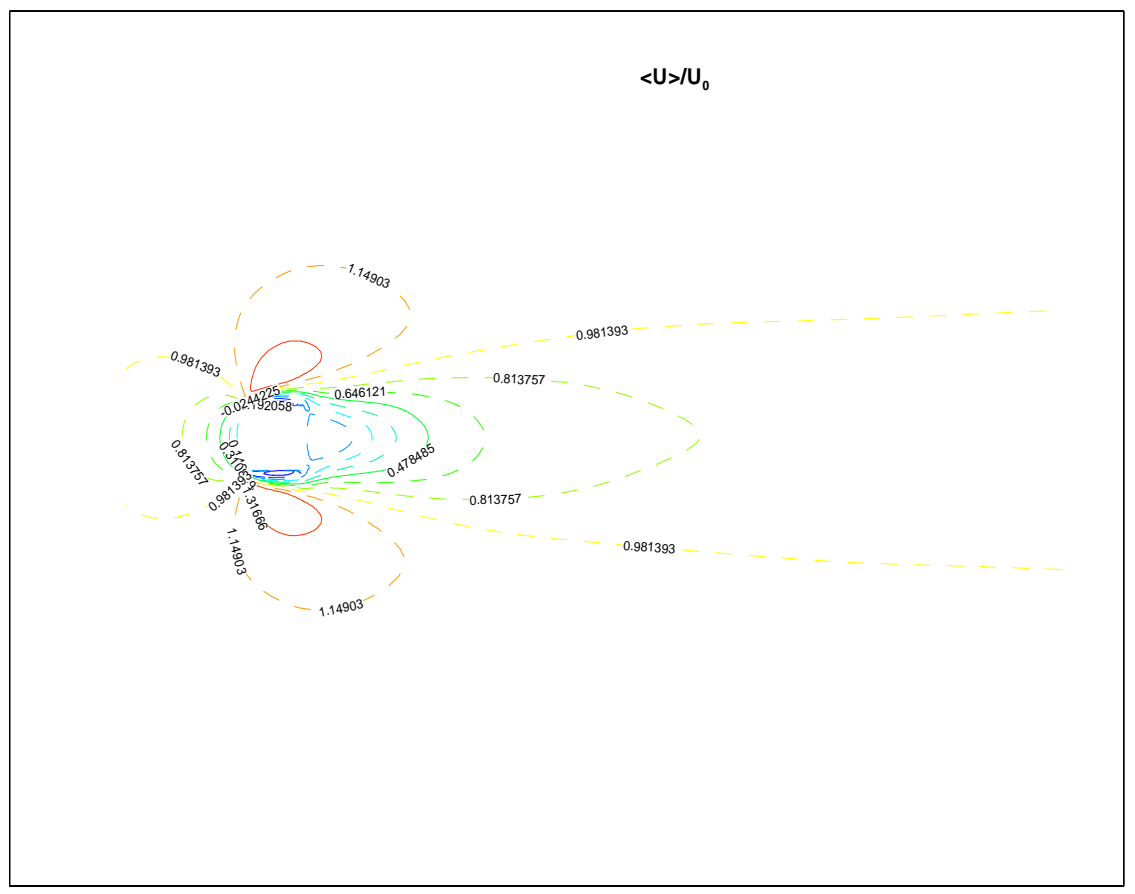

Figure 5-25 Mean streamwise velocity contours (185x185)

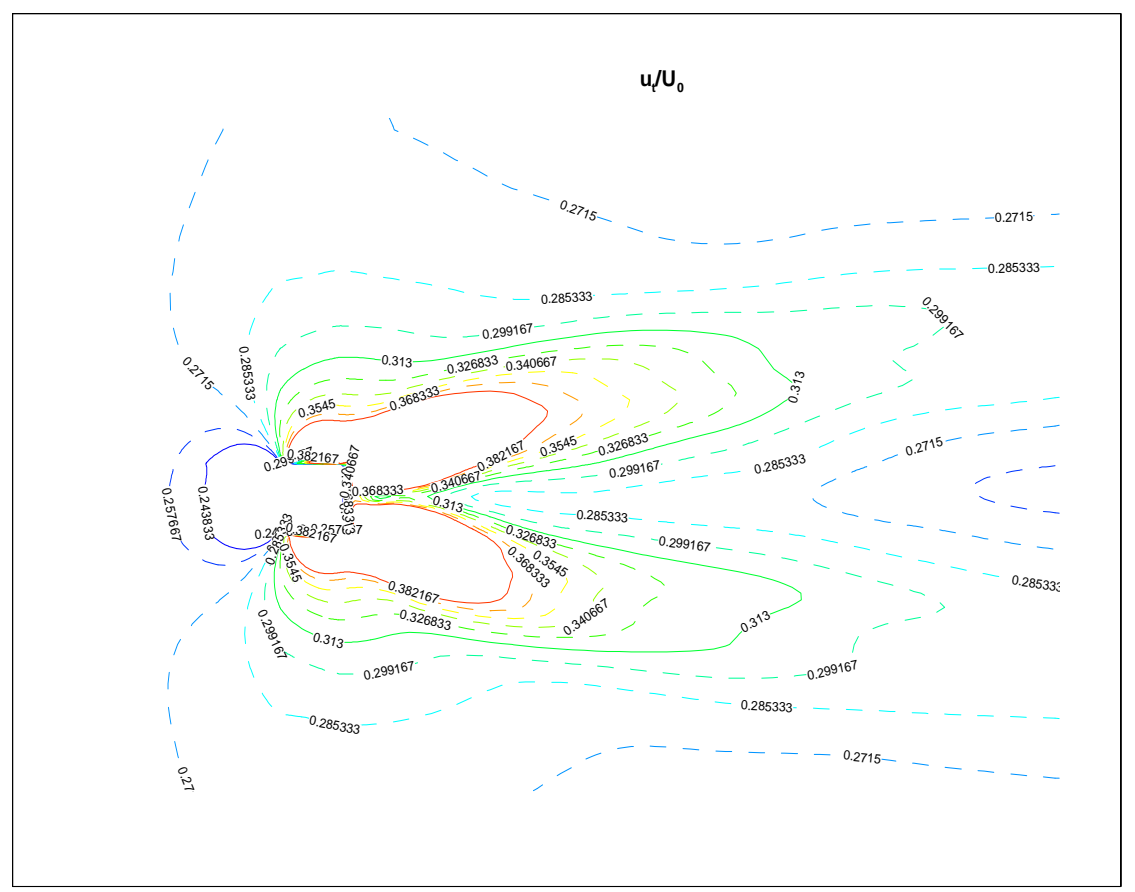

Figure 5-26 Total $u_{\mathrm{rms}}$ contours $(185 \times 185)$ 


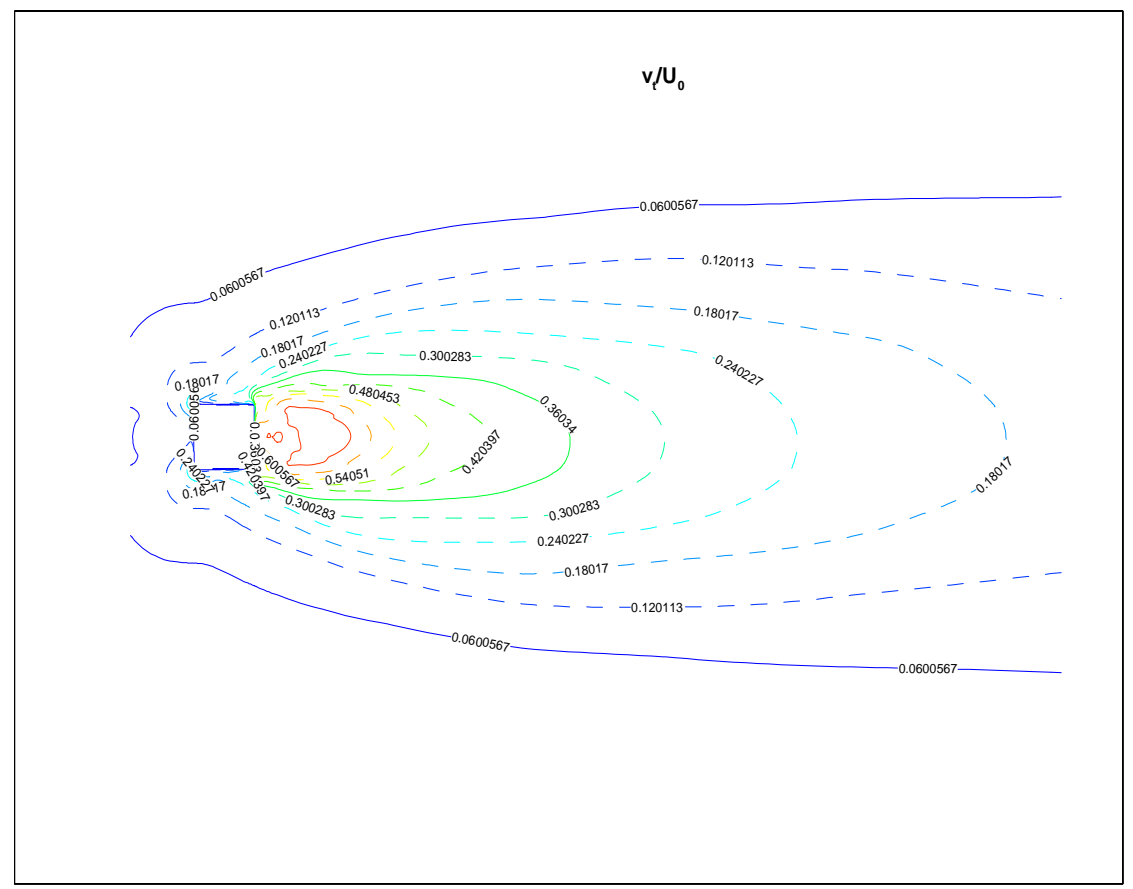

Figure 5-27 Total $v_{\text {rms }}$ contous $(185 \times 185)$

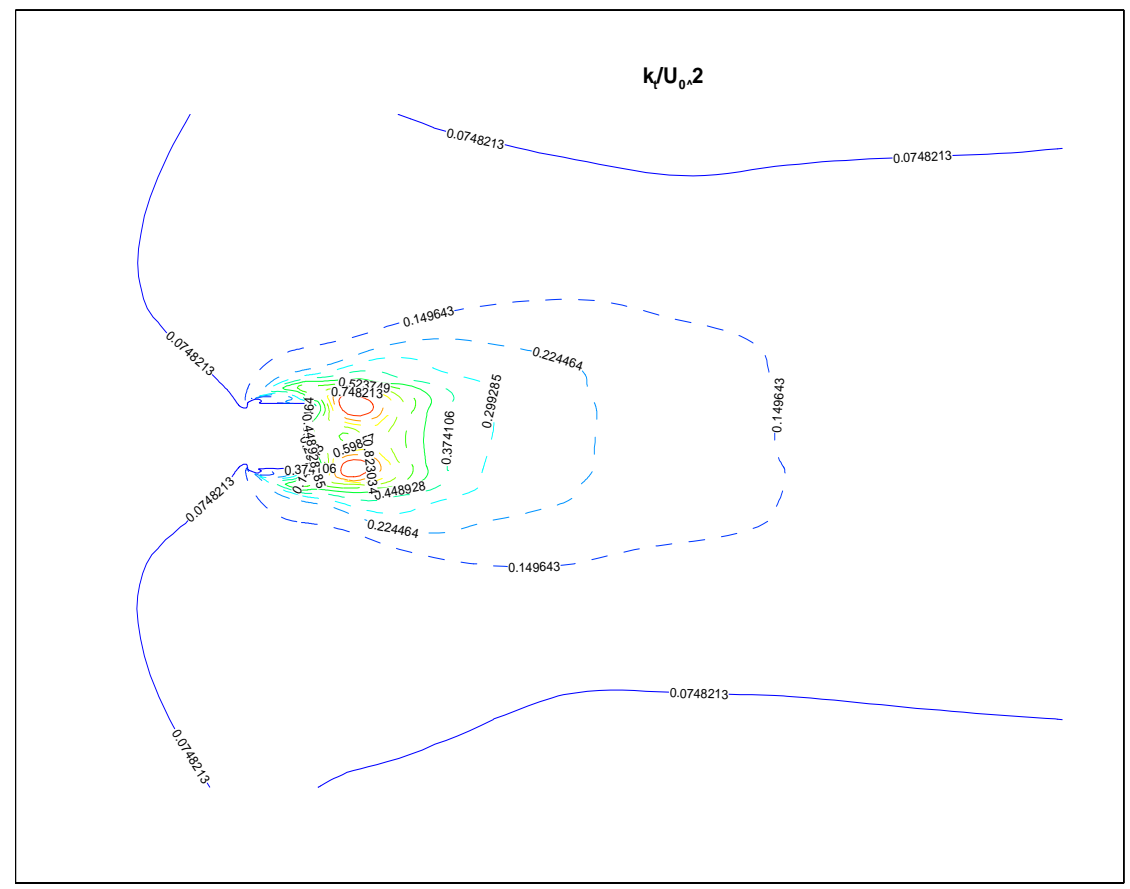

Figure 5-28 Total kinetic energy contours (185x185) 
Figure 5.29 presents the mean velocity along the center-line for the different models as well as experimental data. It is clear that the current model can predict the reattachment length accurately, whereas, the standard k- $\varepsilon$ model predicts a significantly too long recirculation; in the near-field a reasonable good prediction is achieved with the Kato and Launder modification combined with the two-layer approach; the recirculation length is underpredicted with RSM. It is also noticed that only some of the LES calculations from the Rottach-Egern workshop can capture the reattachment length well. The experimental velocity (Lyn et al. 1995) recovers very slowly in the downstream region or even seems to level off at a value of about 0.6 of the upstream free stream level. It seems that the calculations with the current model show a fast recovery in the far-field and the center-line velocity levels off at a high value of 0.83 , which agrees well with the experimental results by Durao, et al. (1988). The LES calculations show a big variance in this region. Two of them predict the recovery satisfactorily but achieve a recirculation that is either too long or too short. The others present the same behavior as the current model, i.e., predict accurate recirculation length but recover too fast.

The total $u_{\mathrm{rms}}$ and $\mathrm{v}_{\mathrm{rms}}$ along center-line are shown in Figure 5.30 and Figure 5.31. The correct trend has been captured by the current model. The maximum relative error to the experimental data is about $20 \%$. 


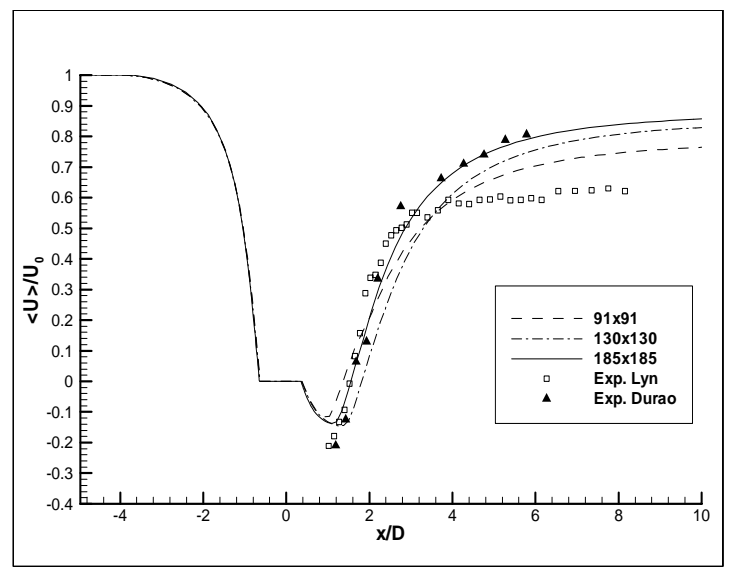

a) present

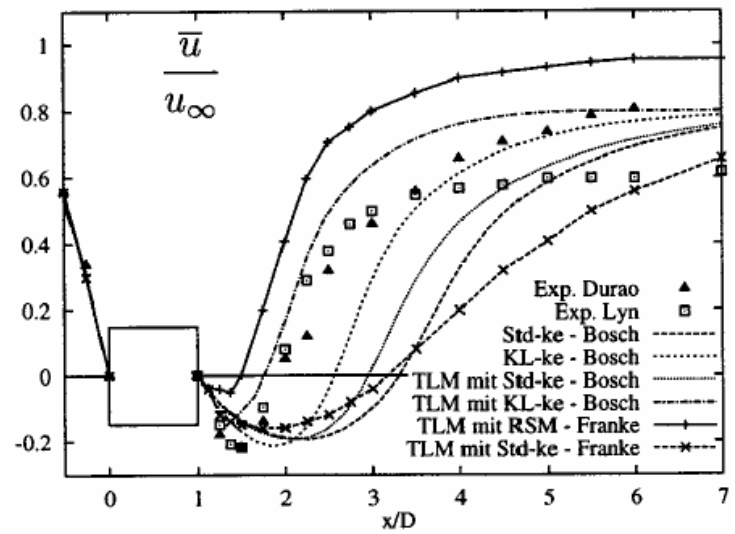

b) RANS calculations (Bosch and Rodi, 1998)

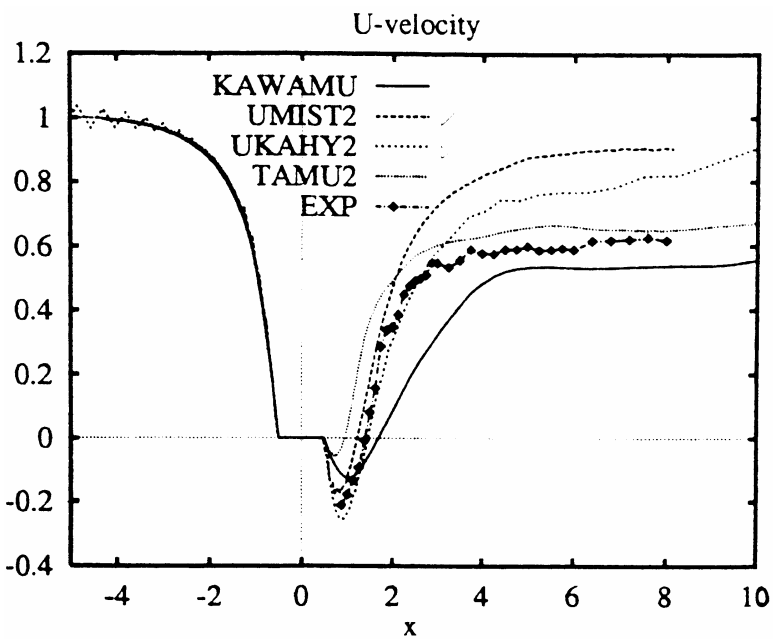

c) LES calculations (Rodi, 2001)

Figure 5-29 Time-mean velocity $<\mathrm{U}>$ along center-line of square cylinder 


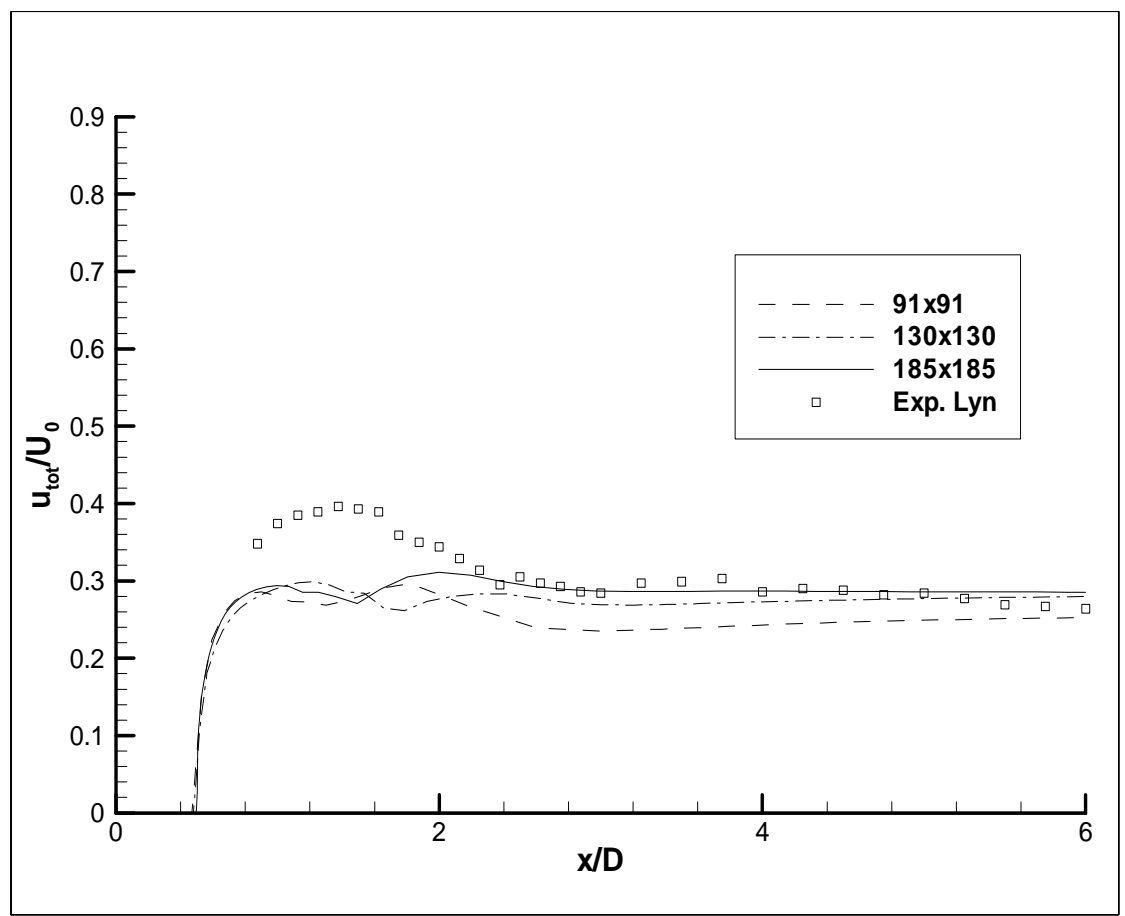

Figure 5-30 Total $u_{r m s}$ along the center-line of the square cylinder

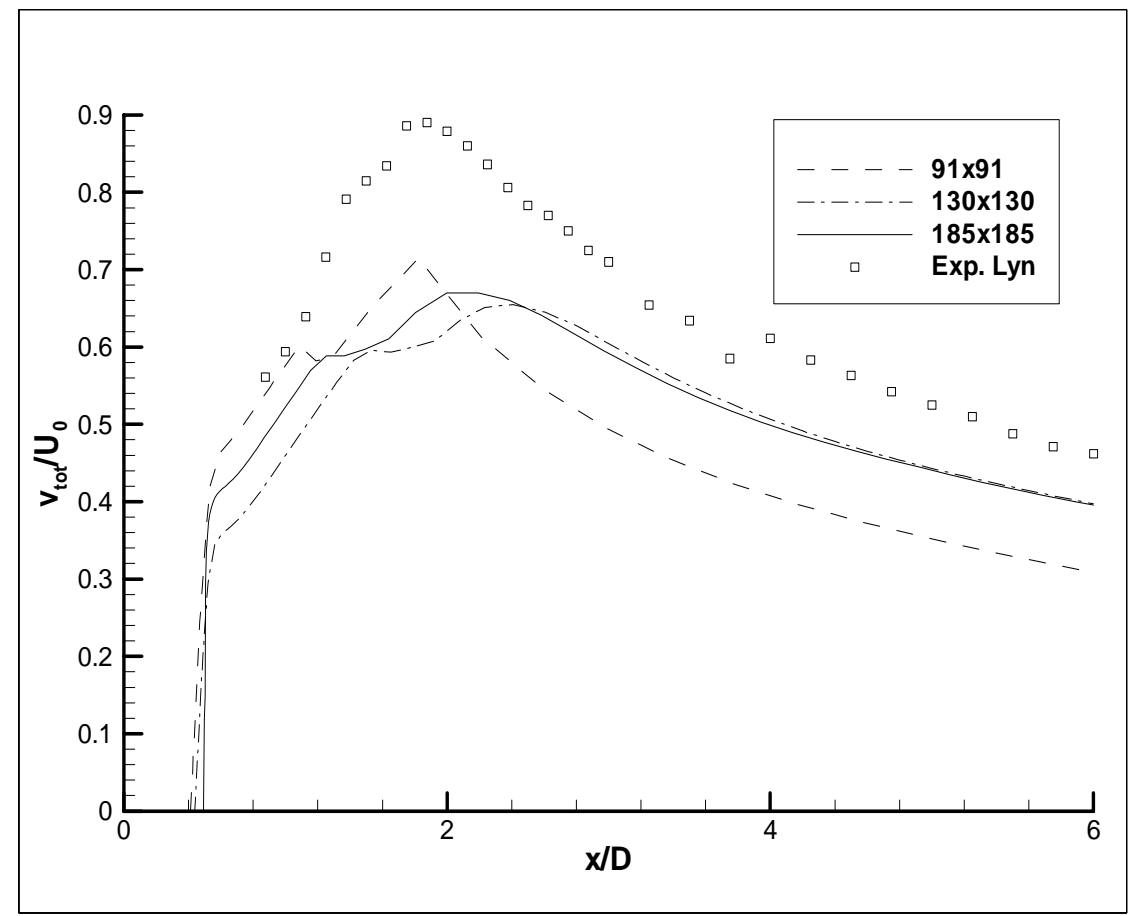

Figure 5-31 Total $v_{\mathrm{rms}}$ along the center-line of the square cylinder 
The total kinetic energy (fluctuation and turbulent) along the center-line is shown in Figure 5.32. The current model shows the same trend as the experiment but an approximately $40 \%$ less at the maximum kinetic energy location. The other RANS models either overpredict the total kinetic energy level or shift the maximum position far downstream. All the LES calculations capture the trend well. Two of them achieve the maximum very close to the experimental data. The others, however, yield comparable results to the current model. The predictions with the coarsest grid (i.e. 91x91) are satisfactory although they are a little different from the ones with finer grids.

Overall, the present model with relatively coarse grid can yield good results for the main quantities of engineering interest, which is superior to some linear turbulence models and one of the Reynolds-stress models shown in the literature (Bosch and Rodi, 1998). As for the flow field and the kinetic energy, the current model achieves results comparable to the LES simulations, and is better than these RANS models used by Bosch and Rodi (1998). 


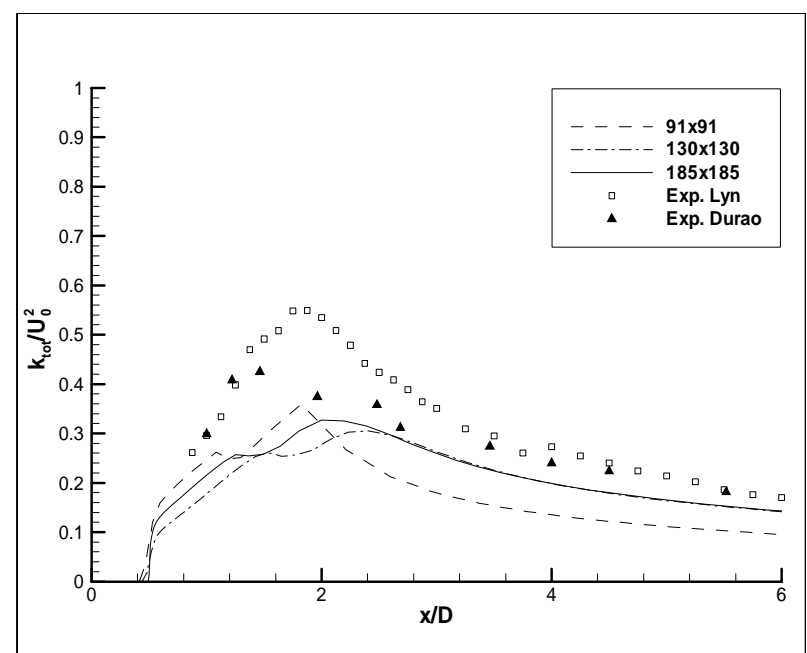

(a) present

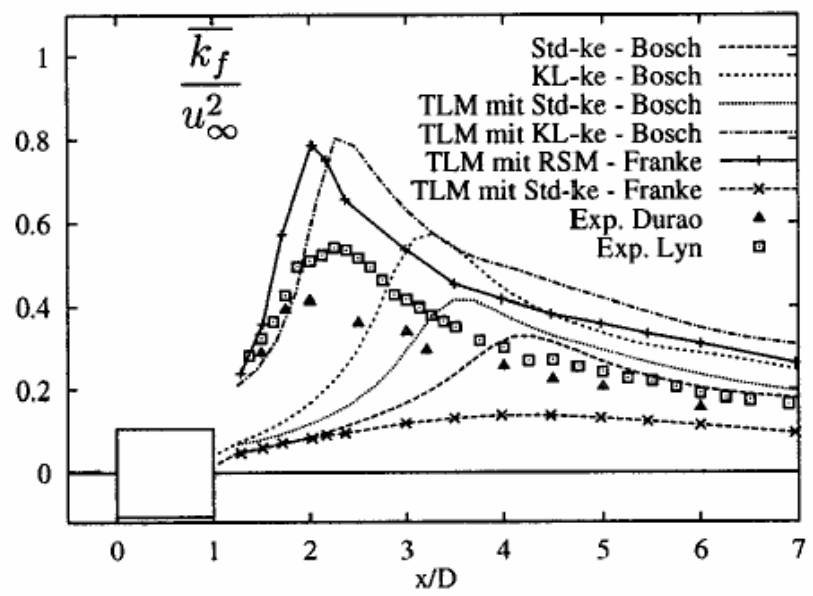

(b) RANS simulations (Bosch and Rodi 1998)

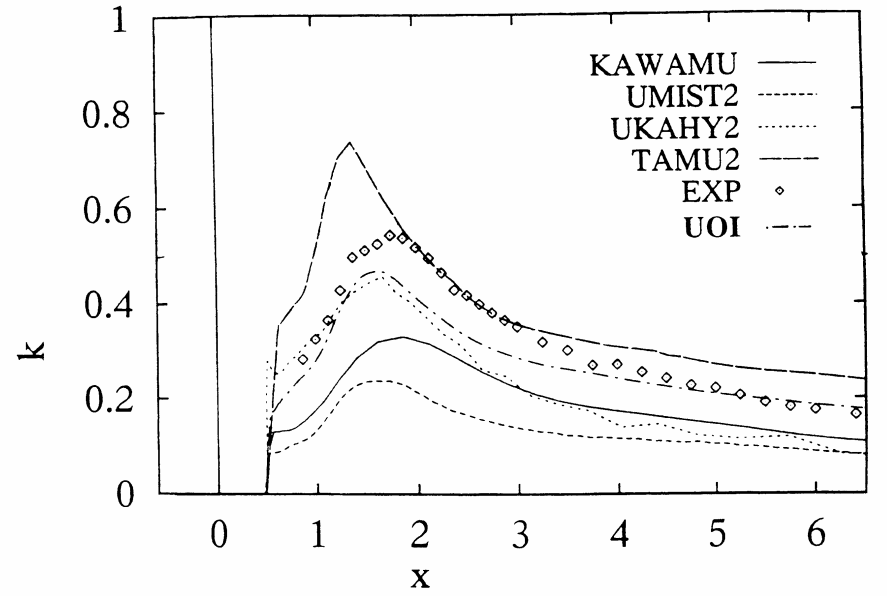

(c) LES simulations (Rodi 2001)

Figure 5-32 Total turbulent kinetic energy along center-line of the square cylinder 


\subsection{Summary}

The cubic low-Reynolds-number model of Craft et al. (1996) was implemented in the DREAM code and applied to turbulent channel flow, flow over a backward facing step, and flow around a square cylinder.

The predictions with the current cubic model on the turbulent channel flow are very close to the ones presented by Craft et al. (1996), which partly verifies the current implementation. The underprediction of the anisotropy near the wall is one of the shortcomings of the current model.

The application on the backward facing step flow with the cubic model exhibits excellent capability of the model. The reattachment length, the velocity profile and the turbulent kinetic energy are all resolved very well with the current model.

For the flow around a long square cylinder, the current model can yield excellent results for the main quantities of engineering interest, which is superior to some linear turbulence models and one of the Reynolds-stress models shown in the literature (Bosch and Rodi, 1998). As for the flow field and the kinetic energy, the predictions with the current model are comparable to the LES simulations, and are more accurate than the ones with other RANS models mentioned in Bosch and Rodi (1998).

Overall, the cubic model is able to accurately resolving the wake flow of a square cylinder, which is comparable to LES simulations. Moreover, the computational cost of the cubic model is much less than the LES. It is strongly recommended to be tested against the cases dealing with airflow and pollutant transport in the wake of bluff body to more fully explore its capabilities. 


\section{Conclusions and recommendations}

\subsection{Conclusions}

In this study, critical issues including body shape, ventilation intensity, free stream turbulence, body heat, and flow unsteadiness were scrutinized for their effect on the air flow and the gaseous pollutant transport in a wind tunnel when the body faces downstream of the flow and pollutant is released from a hand held source pan. Part of the results were compared to experimental data (Guffey, 2005), which has shown good agreement.

The body shape was observed to have a major impact on the flow-field and the concentration field. In fact, this study showed that the predictions with a rounded body, which is one of the closest approximation of a human body (to the best of our knowledge) used in simulations in the exposure assessment literature, results in much lower concentration levels. Simplified body shapes, such as a block and a sharp body, which either block the flow between legs or between the arm and the body, or change the separation profiles, could result in significantly higher exposure levels. This suggests that during modeling oversimplified body shapes should not be used in worker exposure assessment. Specific details, such as the hairstyle, the size and shape of the hat work, the angle of forehead, and the work clothing, may have a significant influence on the exposure and should be considered in certain circumstance. This presents a great challenge to CFD modelers as every worker may exhibit a significant variation on body shape depending on what clothes are worn and factors related to body size and shape.

The effect of the ventilation intensity on the exposure levels with an unheated manikin exhibits a simple pattern, i.e., as ventilation intensifies, exposure declines. However, for a heated manikin, the exposure level reaches its maximum at ventilation intensity in the range of 30-60 fpm $(0.15-0.30 \mathrm{~m} / \mathrm{s})$, where presumably the buoyant force balances the inertial force. At a low ventilation intensity, i.e. 10-20 fpm $(0.05-0.1 \mathrm{~m} / \mathrm{s})$, the buoyancy is dominant. A plume forms and blocks the transport of contaminants from the source pan to the breathing zone, which, in turn, the exposure levels are relatively low. This suggests that the heat flux from the body has a significant impact on the concentration levels and should be considered especially when the convection induced by the buoyancy dominates. 
The heat transfer by radiation from the body is an important way for heat release. Neglecting it may lead to underpredicted exposure levels. It should be noted that the heat flux by sweating/evaporative cooling is not considered in the current experiments and simulations. The evaporative cooling can not only affect the exposure by changing the heat transfer boundary condition, it is also directly related to the comfort level of the worker. Consideration of both the exposure and the comfort level index is desired for today's working environment.

In this study, it was assumed that the heat is released from the upper-body surface to match the experimental conditions. The Grashof number should be the key parameter to understand and estimate the effect of the heat release by the whole body surface or partial body (such as head). Another assumption was that the heat transfer was imposed by either a uniform temperature distribution or a uniform heat transfer distribution. The temperature distribution on a real human body could be significantly non-uniform due to the clothes worn. This also challenges CFD modelers as the clothes worn by the worker may vary significantly.

The effect of the free stream turbulence on the worker exposure was studied since turbulence intensity may vary to a great extent due to different work environment. The results showed that the concentration at the breathing zone reduces as the free stream turbulence intensifies. This could be used in the design of ventilation systems or local ventilation devices to further reduce the exposure levels.

The Eulerian scalar transport model and the Lagrangian trajectory tracking method were compared in their ability to predict the concentrations around a human shaped body exposed to gaseous contaminants within the length of an arm's reach. It was seen that the Eulerian method exhibited a more diffusive nature than the Lagrangian method. However, the concentration predictions obtained with the Lagrangian method may converge with the Eulerian prediction if an adequate number of trajectories are tracked. These models are not limited to predict the concentration of gaseous contaminants, they could also be applied to simulate the transport of aerosol particles.

Nevertheless, the results obtained from both Eulerian and Lagragian predictions suggest that the concentrations measured at the lapel could be very different than the concentrations measured near the mouth (generally, significantly higher). The present study indicates that the latter will be significantly 
lower under the specific conditions pertaining to the current simulations, particularly in the absence of heat flux from the body. However, this conclusion should not be generalized to the cases with uniform concentration distribution, which may be caused by a non-local source or even by the motion of the worker.

The results from this study show that significant differences in predicted concentration levels are observed when different turbulence models are utilized. The coefficient of variation for species concentrations predicted with four different turbulence models ranged between $30-40 \%$. That is why one of the objectives of this study was to recommend a suitable turbulence model for worker exposure studies.

On another note, it was demonstrated that the numerical mesh size and its distribution have significant effects on the quality of predictions. It is recommended that in exposure studies grid convergence of CFD simulations should be assessed in order to reduce and quantify the degree of discretization errors.

To further explore the capability of turbulence models and recommend a suitable turbulence model for worker exposure prediction, a cubic low-Reynolds-number model (Craft et al., 1996 \& 1999) was implemented in DREAM code and applied to turbulent channel flow, flow over a backward facing step, and flow around a square cylinder.

The applications exhibit excellent capability of this model. Overall, it is remarkably superior over linear turbulence models. The results for these cases with this model are even comparable to others' predictions with LES simulations. Moreover, the computational cost of the cubic model is much less than the LES, so it could be a good alternative to serve as a reliable and accurate turbulence model in simulating turbulent flow past a bluff body.

\subsection{Recommendations}

The cubic model is strongly recommended to be tested against the cases dealing with airflow and pollutant transport in the wake of bluff body to fully explore its predictive capability for the present worker exposure studies. To do this, the capability of handling complex geometry should be developed in the DREAM code. Or alternatively, the current model can be connected with a commercial code such as Fluent or Star CD, which usually provide a user defined function module for a user's code. 
Further numerical simulations can be done that include the breathing phenomena and the motion of the worker. An inlet boundary at mouth with a user defined velocity function can be used to present the breathing phenomena. The motion of the worker is more difficult to simulation since it will require the capability of handling the moving boundary for a complex geometry. Advanced algorithms on moving boundary can be developed with the DREAM code and then implemented in commercial codes.

Radiation was not directly modeled and evaporative cooling was not considered in this study. Radiation and evaporation models may be incorporated to predict the temperature distribution in the wake of the human body. Introducing these models would enable us to calculate the comfort level which would be another desirable issue for the work places. 


\section{Bibliography}

Abe, K. and Kondoh, T., and Nagano, Y., 1995, "A new turbulence model for predicting fluid flow and heat transfer in separating and reattaching flows - II. Thermal field calculations," Int. J. Heat Mass Transfer, 38, 1467-1481.

Abe, K., Jang, Y.J., and Leschziner, M.A., 2003, “An investigation of wall-anisotropy expressions and length-scale equations for non-linear eddy-viscosity models," International Journal of Heat and Fluid Flow, 24, 181 - 198.

Achenbach, E., 1968, "Distribution of local pressure and skin friction around a circular cylinder in crossflow up to Re $=5 x 106, " J$. Fluid Mech., 34, 625-639.

Achenbach, E., 1974, “Vortex shedding from spheres,” J. Fluid Mech., 62, 209-221.

Amano, R.S. and Goel, P., 1984, "A numerical study of a separating and reattaching flow by using Reynolds-stress turbulence closure," Numer. Heat Transfer, 7, 343-357.

Apsley, D.D., and Leschziner, M.A., 2000, "Advanced turbulence modeling of separated flow in a diffuser," Flow, Turbulence and Combustion, 63, 81-112

Baldwin, P. E. J. and Maynard A. D., 1998, “A Survey of Wind Speeds in Indoor Workplaces” Ann. Occup. Hyg., 42(5), 303-313.

Bearman, P.W., and Trueman, D.M., 1971, "An investigation of the flow around rectangular cylinders", Aeronaut. Q., 23, 1-6

Bjørn, E. and Nielsen, P.V., 1996, "CFD Models of Persons Evaluated by Full-scale Wind Channel Experiments" Roomvent '96, 2, 137-144.

Bjørn, E. and Nielsen, P. V., 1998, “CFD Simulations of Contaminant Transport Between Two Breathing Persons," Roomvent '98, 2, 133-140.

Bosch, G. and Rodi, W., 1998, "Simulation of Vortex Shedding past a Square Cylinder with Different Turbulence Models," Int. J. Numer. Meth. Fluids, 28, 601-616

Bredberg, J., and Davidson, L., 2004, "Low-Reynolds Number Turbulence Models: An Approach for Reducing Mesh Sensitivity Transactions” ASME Journal of Fluids Engineering, 126, 14-21. 
Brohus, H., and Nielsen, P.V., 1996, “CFD Models of Persons Evaluated by Full-Scale Wind Channel Experiments," Proceedings of Roomvent 96, 5th International Conference on Air Distribution in Rooms, Yokohama, Japan, July 17-19, 2, 137-144.

Bräuer, A., English, M. J. M., Sander, H., Timmermann, A., Braun, U. \& Weyland, W., 2002, "Construction and evaluation of a manikin for perioperative heat exchange," Acta Anaesthesiologica Scandinavica, 46 (1), 43-50.

Cambon, C. and Scott, J.F., 1999, "Linear and nonlinear models of anisotropic turbulence,” Ann. Rev. Fluid Mech., 31, 1-53.

Carlton, G., and Flynn, M.R., 1997, "Field Evaluation of an Empirical-Conceptual Exposure Model,” Appl. Occup.Env. Hyg., 12(8), 555-561.

Celik, I. and Badeau, A. Jr., 2003, "Verification and Validation of DREAM Code," Report No. MAE_IC03/TR103, Mechanical and Aerospace Engineering Department, West Virginia University, Morgantown, WV

Celik, I. and Karatekin, O., 1997, "Numerical Experiments on Application of Richardson Extrapolation with Non-uniform Grids," Journal of Fluids Engineering, 119, 584-590.

Celik, I.B., Li, J., Hu, G., and Shaffer, C., 2005, "Limitations of Richardson Extrapolation and Some Possible Remedies,” To appear on ASME Journal of Fluids Engineering, 127

Celik, I.B. and Li, J., 2005, “Assessment of Numerical Uncertainty for the Calculations of Turbulent Flow over a Backward Facing Step" Accepted for publication in International Journal of Numerical Methods in Fluids, May 2005

Chen, H. C. \& Patel, V. C., 1988, "Near-wall turbulence models for complex flows including separation," AIAA J., 26, 641-648.

Chien, K.Y., 1982, "Predictions of channel and boundary-layer flows with a low-Reynolds number turbulence model," AIAA Journal, 20, 33-38.

Craft, T.J., Launder, B.E., and Suga, K., 1996, "Development and application of a cubic eddy-viscosity model of turbulence," Int. J. Heat and Fluid Flow, 17, 108-115. 
Craft, T.J., Iacovides, H., Yoon, J.H., 1999, "Progress in the use of non-linear two-equation models in the computation of convective heat-transfer in impinging and separated flows," Flow, Turbulence \& Combustion, 63, 59-80.

Dejoan, A. and Leschziner, M.A., 2004, "Large Eddy Simulation of periodically perturbed separated flow over a backward-facing step," Int. J. Heat and Fluid Flow, 25, 581-592.

Durao, D.F.G., Heitor, M.V., and Pereira, J.C.F., 1988, "Measurements of turbulent and periodic flows around a square cross section cylinder," Experiments in Fluids, 6, 298-304.

Durbin, P. A., 1991, "Near-wall turbulence closure modeling without 'damping functions," Theoret. Comput. Fluid Dyn., 3, 1-13.

Durbin, P. A., 1993, “A Reynolds stress model for near-wall turbulence,” J. Fluid Mech., 249, 465-498.

Durbin, P.A., 1995, “Separated Flow Computations with the k- $\varepsilon-v 2$ Model,” AIAA Journal., 33, $659-664$

Ercoftac Database, www.ercoftac.org, last accessed on July 15th 2005.

Flynn, M.R. and Ljungqvist B., 1995, “A Review of Wake Effects on Worker Exposure,” Ann. Occup. Hyg., 39(2), 211-221.

Flynn, M.R., and Sills, E., 2000, “On the use of Computation Fluid Dynamics in the Prediction and Control of Exposureto Airborne Contaminants - an illustration using Spray Painting," Ann. occup. Hyg., 44(3), 191-202.

Flynn, M.R. and Sills, E.D., 2001, "Numerical Simulation of Human Exposure to Aerosols Generated during Compressed Air Spray-Painting in Cross-Flow Ventilated Booths," Journal of Fluids Engineering, 123, 64-70.

Franke, R. and Rodi, W., 1993, "Calculation of vortex Shedding Past a Square Cylinder with Various Turbulence Models," Turbulent Shear Flows, 8, F. Durst et al., eds., Springer, New York, 189-204.

Frisch, U., 1995, Turbulence, Cambridge University Press.

Fu, S., Launder, B.E. and Tselepidakis, D.P., 1987, “Accommodating the effects of high strain rates in modeling the pressure-strain correlation," Mechanical Engineering Department Report TFD/87/5, UMIST.

George, D., Flynn, M.R., and Goodman, R., 1990, “The Impact of Boundary Layer Separation on Local Exhaust Design and Worker Exposure,” Appl. Occup.Env. Hyg., 5(8), 501-509. 
Gibson, M.M., and Launder., B.E., 1978, "Ground Effects on Pressure Fluctuations in the Atmospheric Boundary Layer,” J. Fluid Mech., 86, 491-511.

Guffey, S.E., Flanagan, M.E., and van Belle, G., 2001, "Air Sampling at the Chest and Ear as Representative of the Breathing Zone," AIHA J., 62, 416-427.

Guffey, S.E., 2004-2005, private communication

Hancock, P.E., and Bradshaw, P., 1983, “The effect of free-stream turbulence on turbulent boundary layers," Journal of Fluids Engineering, 105, 284-289.

Hancock, P.E., and Bradshaw, P., 1989, “Turbulence structure of a boundary layer beneath a turbulent free stream," Journal of Fluids Engineering, 205, 45-76.

Hanjalic, K., 1970, “Two-dimensional asymmetric flows in ducts,” Ph.D. Thesis, University of London

Hanjalic, K. and Launder, B.E., 1972, “A Reynolds stress model of turbulence and its application to thin shear flows," J. Fluid Mech., 52, 609-638.

Hanjalic, K. 2002 “One-Point Closure Models for Bouyancy-Driven Turbulent Flows”, Annu. Rev. Fluid Mech. 34, 321-347.

Hanjalic, K., Popovac, M. and Hadziabdic, M., 2004, “A robust near wall elliptic-relaxation eddy-viscosity turbulence model for CFD," International Journal of Heat and Fluid Flow, 25, 1047-1051.

Hanjalic, K., 2004, “Will RANS Survive LES? A view of Perspectives,” Proceedings of ASME HT-FED 04 2004 ASME Heat Transfer/Fluid Engineering Summer Conference, July 11-15, Charlotte, North Carolina, USA.

Harlow, F.H., 1968, “Transport of turbulence energy decay rate,” University of California Report LA-3845, Los Alamos Science Laboratory.

Hjelmfelt Jr., A. T., and Mockros, L. F., 1966, "Motion of discrete particles in a turbulent fluid," Appl. Sci. Res., 16, 149-161.

Hoffman, G.H., 1975, “Improved form of low Reynolds-number k- $\varepsilon$ turbulence model," Physics of Fluids, 18, 309-312.

Hyun, S. and Kleinstreuer, S., 2001, "Numerical Simulation of Mixed Convection Heat and Mass Transfer in a Human Inhalation Test Chamber," International Journal of Heat and Mass Transfer, 44, 22472260. 
Huang, P.G. and Leschziner, M.A., 1985, "Stability of recirculation flow computations performed with second-moment closures and third-order discretization," Proc. 5th Turbulent Shear Flows Conf., Ithaca, NY

Hwang, R.R and Peng, Y.F., 1995, "Computation of Backward-facing Step Flows by a Second-order Reynolds Stress closure model," International Journal for Numerical Methods in Fluids, 21, 223235.

Iaccarino, G., and Durbin, P.A., 2000, "Unsteady 3D RANS Simulation Using the V2F Model," CTR Annual Research Brief, Stanford University, November.

Iacovides, H., Raisee, M., 1997, "Computation of flow and heat transfer in 2D rib roughened passages," Proc. 2nd Int. Symp. on Turbulence, Heat and Mass Transfer, Delft.

Iacovides, H., and Raisee, M., 1999, "Recent Progress in Computation of Flow and Heat Transfer in Internal Cooling Passages of Turbine Blades," Int. J. of Heat \& Fluid Flow, 20, 320-328.

Johns, W.P. and Launder, B.E., 1972, "The prediction of laminarization with a two-equation model of turbulence," Int. J. Heat and Mass Transfer. 15, 301-314.

Jones, W.P. and Musonge, P., 1988, "Closure of the Reynolds stress and scalar flux equations," Phys. Fluids, 31, 3589-3604.

Kassinos, S.C., Langer, C.A., Haire, S.L. and Reynolds, W.C., 2000, "Structure-based modeling for wallbounded flows," Int. J. Heat and Fluid Flow, 21, 599-605.

Kato, M. and Launder, B. E., 1993, “The Modelling of Turbulent Flow around Stationary and Vibrating Square Cylinders," Proc. 9th Symposium on Turbulent Shear Flows, Kyoto.

Kawamura, H., and Kawashima, N.,1994, "A proposal for a k-E model with relevance to the near-wall turbulence,” Paper IO.1, Proc. Int. Symp. On turbulence, Heat and Mass Transfer, Lisbon

Kim, J., Moin, P., 1985, “Application of a fractional step-method to incompressible navier-stokes equations.," J. Comput. Phys., 59(2), 308-323.

Kim, J., Moin, P., and Moser, R., 1987, "Turbulence statistics in fully developed channel flow at low Reynolds number," J. Fluid Mech., 177, 133-166.

Kim, T. and Flynn, M.R., 1991, “Air Flow Patterns Around a Worker in a Uniform Freestream,” Am. Ind. Hyg. Assoc. J., 52(7), 287-296. 
Kondjoyan, A., Péneau, F., and Boisson, H.C., 2002, “Effect of high free-stream turbulence on heat transfer between plates and air flows : A review of existing experimental results," Int. J. Therm. Sci., 41, 116.

Kulmala, I, Säämänen, A. and Enbom, S., 1996, “The Effect of Contaminant Source Location on Worker Exposure in the near-wake region," Ann.Occup.Hyg., 40, 511-523.

Lam, C.K.G., and Bremhorst, K., 1981, "A modified form of the k- $\varepsilon$ model for predicting wall turbulence,” Journal of Fluids Engineering, 103, 456-460.

Lan, N.S., and Viswanathan, S., 2001, "Numerical simulation of airflow around a variable volume/constant face velocity fume cupboard," AIHAJ. 62(3), 303-312.

Launder, B.E., and Sharma, A., 1974, “Application of the Energy-Dissipation Model of Turbulence to the Calculation of Flow Near a Spinning Disk," Lett. Heat Mass Transfer, 1, 131-138.

Launder, B.E. and Spalding, D.B., 1974, “The Numerical Computation of Turbulent Flows," Computer Methods in Applied Mechanics and Engineering, 3, 269-289.

Launder, B.E., Reece, G.J. and Rodi, W., 1975, "Progress in the development of a Reynolds-stress turbulence closure," J. Fluid Mech., 68, 537-566.

Launder, B.E., 1989, "Second-moment Closure and its Use in Modeling Turbulent Industrial Flows," International Journal for Numerical Methods in Fluids, 9, 963-985.

Le, H., and Moin, P., 1992, "Direct numerical simulation of turbulent flow over a backward-facing step ," Stanford Univ., Center for Turbulence Research, Annual Research Briefs, 161-173.

Le, H., Moin, P. and Kim, J., 1997, "Direct numerical simulation of turbulent flow over a backward-facing step," J. Fluid Mech., 330, 349-374.

Lee, M.J., Kim, J. and Moin, P., 1990, "Structure of turbulence at high shear rate," J. Fluid Mech., 216, 561-583.

Leonard, B. P., 1979, “A stable and accurate convective modelling procedure based on quadratic upstream interpolation," Computer Methods in Applied Mechanics and Engineering, 19, 59-98.

Li, J., Yavuz, I., Celik, I.B., and Guffey, S.E., 2005, “A Numerical Study of Worker Exposure to a Gaseous Contaminant: Variations on Body Shape and Scalar Transport Model," Journal of Occupational and Environmental Hygiene, 2,323 - 334 
Li, J., Yavuz, I., Celik, I.B., Guffey, S.E. and Bird, A., 2003, "The Effect of Turbulence and Scalar Transport Models on Prediction of Worker Exposure to Aerosols," Proceedings of FEDSM'03, 4TH ASME_JSME Joint Fluids Engineering Conference, Honolulu, Hawaii, USA, July 6-11

Lien, F.S. and Leschziner, M.A., 1994, "Modeling the flow in a transition duct with a non-orthogonal FV procedure and low-Re turbulence-transport models," Proc. ASME FED Summer Meeting, Symposium on Advances in Computational Methods in Fluid Dynamics, 93-106.

Lien, F.S. and Leschziner, M.A., 1994, "Assessment of turbulence -transport models including non-linear RNG eddy-viscosity formulation and second-moment closure for flow over a backward facing step" Computers Fluids, 23, 8, 983-1004.

Lien, F.S., Kalitzin, G., and Durbin, P.A., 1998, "RANS modeling for compressible and transitional flows," Center for Turbulence Research, proceedings of the Summer Program 1998.

Lilly, D.K., 1966, "In the Application of the Eddy Viscosity Concept in the Inertial Subrange of Turbulence," NCAR Manuscript 123.

Longest, P.W., Kleinstreuer, C. and Kinsey, J.S., 2000, “Turbulent Three-dimensional Air Flow and Trace Gas Distribution in an Inhalation Test Chamber,” Journal of Fluids Engineering, 122, 403-411.

Lyn, D.A., and Rodi, W., 1994, "The unsteady shear layer formed by flow separation from the forward corner of a square 2-D cylinder," Journal of Fluid Mechanics, 267, 353-396.

Lyn, D.A., Einav, S., Rodi, W., and Park, J.H., 1995, “A laser Doppler velocimetry study of ensembleaveraged characteristic of the turbulent flow near wake of a square cylinder," J. Fluid Mech, 304, $285-319$.

Mathieu, J. and Scott, J., 2000, An Introduction to Turbulent Flow, Cambridge University Press.

Menter, F.R., 1994, "Two-Equation Eddy-Viscosity Turbulence Models for Engineering Applications," AIAA Journal, 32(8), 1598-1605.

Mills, A.F., 1999, Basic Heat and Mass Transfer, Prentice-Hall.

Moser, R. D., Kim, J., and Mansour, N. N., 1999, "Direct numerical simulation of turbulent channel flow up to $\mathrm{Re}_{\tau}=590, "$ Phys. Fluids, 11, 943-945.

Myong, H.K., and Kasagi, N., 1990, “A new approach to the improvement of k- $\varepsilon$ turbulence model for wall bounded shear flows," JSME Int. J., SeriesII, 33, 63-72. 
Myong, H.K. and Kasagi, N., 1990, "Prediction of anisotropy of the near wall turbulence with an anisotropic low-Reynolds number k- $\varepsilon$ turbulence model," J.Fluids Eng., 112, 521-524

Nagano, Y. and Hishida, M., 1987, "Improved form of the k- $\varepsilon$ model for turbulent shear flows," Trans. of ASME. 109, 156.

Nisizima, S. and Yoshizawa, A., 1987, “Turbulent channel and Couette flows using an anisotropic k- $\varepsilon$ model," AIAA J., 25, 414-420

Orszag, S.A., Yakhot, V., Flannery, W.S., Boysan, F., Choudhury, D., Maruzewski, J., and Patel, B., 1993, "Renormalisation group modeling and turbulence simulations," Near-Wall Turbulent Flows, (R.M.C. So, C.G. Speziale and B.E. Launder, Eds.), Elsevier, 1031-1046

Patel, V. C., Rodi, W. \& Scheuerer, G., 1985, "Turbulence Models for Near-Wall and Low Reynolds Number Flows: A Review,” AIAA J., 23, 1308-1319.

Péneau, F., Boisson, H.C., and Djilali, N., 2000, "Large eddy simulation of the influence of high freestream turbulence on spatially evolving boundary layer," Int. J. of Heat and Fluid Flow, 21, 640647.

Pope, S.B., 1975 “A more general effective-viscosity hypothesis”, J. Fluid Mech., 72, 331-340.

Raisee, M., Noursadeghi, A., and Iacovides, H., 2004, “Application of a non-linear k- $\varepsilon$ model in prediction of convective heat transfer through ribbed passages," Int. J. of Numerical Methods for Heat \& Fluid Flow, 14, 285-304.

Roache, P.J., 1994, "Perspective: A Method for Uniform Reporting of Grid Refinement Studies,” Journal of Fluids Engineering, 116, 405-413.

Rodi, W., 2001, "Large-Eddy Simulation of flow past bluff bodies," Closure Strategies for Turbulent and Transitional Flows, 361-391.

Rodi, W., and Mansour, N.N., 1993, “Low Reynolds number k- $\varepsilon$ modeling with the aid of direct numerical simulation data," Journal of Fluid Mech., 250, 509-529.

Rodi, W., Mansour, N.N., and Michelassi, V., 1993, “One-Equation Near-Wall Turbulence Modeling With the Aid of Direct Simulation Data, ” ASME J. Fluids Eng., 115, 196-205.

Rodi, W., Ferziger, J. H., Breuer, M., and Pourquie, M., 1997, "Status of Large Eddy Simulation: Result of a Workshop,” J. Fluids Eng., 119, 248-262. 
Rotta, J.C., 1951, “Statistische Theorie nichthomogener Turbulenz,” Z. Phys., 129, 547-572.

Rubinstein, R. and Barton, J.M., 1990, "Non-linear Reynolds stress models and renormalization group," Phys. Fluids, A2, 1472-1476.

Saamanen, A., Kulmala, I., Welling, I., Rosen, G., and Anderson I.M., 2000, "Person in a Uniform Airflow - Effects of Freestream Air Velocity and Body Convection," Proceedings of the Ventilation 2000, 6th International Symposium on Ventilation for Contaminant Control, 4-7 June, Helsinki, Finland.

Saga, T., Hu, H., Kobayashi, T., Murata, S. 0, Okamoto, K.and Nishio, S., 2000, “A Comparative Study of the PIV and LDV Measurements on a Self-induced Sloshing Flow,” Journal of Visualization, 3, $145-156$.

Schiestel, R., 1987, "Multiple time scale modeling of turbulent flows in one point closures," Phys. Fluids. 30722.

Shih, T. H., and Lumley, J. L., 1985, "Modeling of pressure correlation terms in Reynolds stress and scalar flux equation,” Tech. Rep. FDA-85-3, Cornell University.

Shih, T.H., Zhu, J. and Lumley, J.L., 1993, “A realizable Reynolds stress algebraic equation model,” NASA tech. memo. 105993.

Smagorinsky, J., 1963, "General Circulation Experiments with the Primitive Equations. I. The Basic Experiment," Month. Wea. Rev., 91, 99-164.

So, R.M.C., Zhang, H.S. and Speziale, C.G., 1991, "Near-wall modeling of the dissipation rate equation," AIAA Journal, 29, 2069-2076.

Spalat, P.R., 2000, “Strategies for turbulence modeling and simulations," International Journal of Heat and Fluid Flow, 21, 252-263.

Speziale, C.G., 1987, “On non-linear k-l and k- $\varepsilon$ models of turbulence,” J. Fluid Mech., 178, 459-475.

Speziale, C.G., Sarkar, S. and Gatski, T.B., 1991, "Modelling the pressure-strain correlation of turbulence: an invariant dynamical systems approach,” J. Fluid mech., 227, 245-272.

Spiegel, E. A., and Veronis, G., 1960, “On the Boussinesq approximation for a compressible fluid," The Astrophysical Journal, 131, 442-447.

Suga, K., 1995, "Development and application of a non-linear eddy viscosity model sensitized to stress and strain invariants," PhD Thesis, Faculty of Technology, University of Manchester. 
Sveningsson, A., 2003, “Analysis of the performance of Different V2-F turbulence models in a stator vane passage flow," Thesis for the Degree of Licentiate in Engineering.

Taneda, S., 1956, "Experimental investigation of the wake behind a sphere at low Reynolds number," $J$. Phys. Soc. Japan, 11, 1104-1108.

Taneda, S., 1978, "Visual observations of the flow past a circular cylinder performing a rotary oscillation," J. Phys. Soc. Japan, 45, 1038-1043.

Tritton, D. J., 1959, “Experiments on the flow past a circular cylinder at low Reynolds numbers," Journal of Fluid Mechanics, 6(4), 547

van Driest, E.R., 1956, “On Turbulent Flow Near a Wall,” Journal of the Aeronautical Sciences, 23, 10071011.

Voke, P.R., Chollet, J.P., and Kleiser, L., 1997, Direct and Large Eddy Simulation II, Kluwer Academic Publisher.

Wilcox, D.C., 1988, "Multi-scale model for turbulent flows," AIAA J., 26, 1311-1320.

Welling, I., Andersson, I.M., Rosen, G., Raisanen, J., Mielo, T., Marttinen K., and Niemela, K., 2000, "Contaminant Dispersion in the Vicinity of a Worker in a Uniform Velocity Field," Ann. Occup. Hyg. 44(3), 219-225.

Yakhot, V. and Orszag, S.A., 1986, "Renormalization Group Analysis of Turbulence. I. Basic Theory,” J. of Sci. Comp., 1, 3-51

Yap, C., 1987, "Turbulent Heat and Momentum Transfer in Recirculating and Impinging Flows," Ph.D. Thesis, University of Manchester, UK. 


\section{Appendix A. Methods for Numerical Uncertainty Estimation}

\section{(1) Least squares method}

With the least squares approach, $\phi_{0}\left(\cong \phi_{e x t}\right), \alpha$, and $\mathrm{p}$ are computed by minimizing the following function.

$$
\sigma\left(\phi_{0}, \alpha, p\right)=\sqrt{\sum_{i=1}^{n}\left(\phi_{i}-\phi_{0}-\alpha h_{i}^{p}\right)^{2}}
$$

where $\mathrm{n}$ is the number of grids available. The minimum of (A.1.1) is found by setting the derivatives of (A.1.1) with respect to $\phi_{0}, \alpha$, and $\mathrm{p}$ equal to zero, which leads to a non-linear system of equations. Solving the non-linear system yields values for $\phi_{0}, \alpha$, and $\mathrm{p}$.

\section{(2) Polynomial method}

This method uses the first few terms in the Taylor expansion of $\phi(h)$ to approximate $\phi(h)$. For instance, assuming the method is first-order, the first three terms are used if a set of 3 grids are available. That is

$$
\phi(h)=\phi(0)+a_{1} h+a_{2} h^{2}
$$

If 4 grids are available, the first four terms are used

$$
\phi(h)=\phi(0)+a_{1} h+a_{2} h^{2}+a_{3} h^{3}
$$

If the scheme is higher order $(\mathrm{p} \geq 2)$, this method will mean essentially a curve fit to the actual error function. For a fourth order method one has to keep at least 4 terms, i.e. five sets of calculations are needed.

The extrapolation to the limit approach is recommended to solve the equations formed by polynomial method. This approach uses the following formula to calculate the extrapolated solution $\phi^{(3)}(h)$ for 3 grids and $\phi^{(4)}(h)$ for 4 grids. 


$$
\phi^{(m)}(h)=\frac{\phi^{(m-1)}(\alpha h)-\alpha^{m} \phi^{(m-1)}(h)}{1-\alpha^{m}}
$$

$\mathrm{m}=1,2, \cdots$

It is easy to tabulate the sequential steps of the calculation procedure and to add more points later.

\section{(3) Power law method}

The Power law method proposed by Celik and Karatekin(1997) are used for 3 grids. The idea follows

$$
\begin{aligned}
& \phi(0)-\phi\left(h_{1}\right)=c h_{1}^{p} \\
& \phi(0)-\phi\left(h_{2}\right)=\operatorname{sign}\left(\frac{\varepsilon_{32}}{\varepsilon_{21}}\right) c h_{2}^{p} \\
& \phi(0)-\phi\left(h_{3}\right)=c h_{3}^{p}
\end{aligned}
$$

where $\varepsilon_{32} / \varepsilon_{32}=\left(\phi\left(h_{3}\right)-\phi\left(h_{2}\right)\right) /\left(\phi\left(h_{2}\right)-\phi\left(h_{1}\right)\right)$ the sign of which is positive for monotonic convergence and negative for non-monotonic convergence. There are 3 unknowns, $\phi(0), \mathrm{c}$, and $\mathrm{p}$. The same iterative method to solve (A.3.1)-(A.3.3) as done by Celik and Karatekin (1997) is implemented.

For 4 grids, the following can be applied

$$
\phi\left(h_{i}\right)-\phi(0)=a_{1} h_{i}^{p}+a_{2} h_{i}^{p+1} \quad i=1,2,3,4
$$

Non-monotonic convergence is facilitated if $\mathrm{a}_{1}$ and $\mathrm{a}_{2}$ are of opposite sign. It should be noted that for some cases there is no solution to Eq. (A.3.4). Those cases will be counted as unsuccessful outcomes.

\section{(4) Cubic spline method}

The well known natural cubic splines curve fitting technique is used to create the cubic splines between three points or four points. $\phi(0)$ can be found by extrapolating the curve for the interval closest to $h=0$. 


\section{(5) Approximate error spline (AES) method}

Still using Taylor series expansion for $\phi(h)$ and substituting $\alpha h$ for $h$, one obtains

$$
\phi(\alpha h)=\phi(0)+a_{1} \alpha h+a_{2}(\alpha h)^{2}+a_{3}(\alpha h)^{3}+\cdots
$$

The true error $\mathrm{E}_{\mathrm{t}}$ is given by

$$
E_{t}(\alpha, h) \equiv \phi(\alpha h)-\phi(0)=\sum_{k=1}^{\infty} a_{k} \alpha^{k} h^{k}
$$

and the approximate error Ea

$$
E_{a}(\alpha, h) \equiv \phi(\alpha h)-\phi(h)
$$

where $E_{t}(\alpha, h)$ is the true error and $E_{a}(\alpha, h)$ is the approximate error which presents the difference of the subsequent results with the fine grid and the coarse grid. So one has

$$
E_{a}(\alpha, h)=\sum_{k=1}^{\infty} a_{k}\left(\alpha^{k}-1\right) h^{k}
$$

Dividing (A.5.1) by (A.5.3) and moving $E_{a}(\alpha, h)$ to the right hand side yield

$$
E_{t}(\alpha, h)=\frac{1}{1-\frac{\sum a_{k} h^{k}}{\sum a_{k} \alpha^{k} h^{k}}} E_{a}(\alpha, h)
$$

letting

$$
\frac{\sum a_{k} h^{k}}{\sum a_{k} \alpha^{k} h^{k}}=b_{0}+b_{1} h+b_{2} h^{2}
$$

and expanding the 1.h.s. of the above equation and comparing it with the r.h.s. give 


$$
\begin{aligned}
& b_{0}=\frac{1}{\alpha} \quad b_{1}=\left(\frac{1-\alpha}{\alpha}\right) \frac{a_{2}}{a_{1}} \\
& b_{2}=\left(\frac{1-\alpha^{2}}{\alpha}\right) \frac{a_{3}}{a_{1}}-(1-\alpha)\left(\frac{a_{2}}{a_{1}}\right)^{2}
\end{aligned}
$$

Now Eq. (A.5.4) can be rewritten as

$$
E_{t}(\alpha, h)=\frac{1}{1-\left(b_{0}+b_{1} h+b_{2} h^{2}\right)} E_{a}(\alpha, h)
$$

In order to calculate $b_{0}, b_{1} \& b_{2}$, one needs to calculate $a_{1}, a_{2}, \& a_{3}$ first. It is seen from Eq. (A.5.3) that

$$
a_{k}=\frac{E_{a}{ }^{(k)}(\alpha, 0)}{k !\left(\alpha^{k}-1\right)} \quad k=1,2,3
$$

$\mathrm{E}^{(\mathrm{k})}$ is the $\mathrm{k}^{\text {th }}$ derivative of $\mathrm{E}$. Assuming that one has 3 grids and the solutions as $\left(h_{1}, \phi\left(h_{1}\right)\right)$, $\left(h_{2}, \phi\left(h_{2}\right)\right)$ and $\left(h_{3}, \phi\left(h_{3}\right)\right)$ with $h_{3}=\alpha h_{2}=\alpha^{2} h_{1}$. And noting that $E_{a}(\alpha, 0) \equiv 0$ leads to 3 points as $\left(h_{1}, E_{a}\left(\alpha, h_{1}\right)\right),\left(h_{2}, E_{a}\left(\alpha, h_{2}\right)\right)$ and $\left(0, E_{a}(\alpha, 0)\right)$ which involves the approximate error instead of the numerical solution $\widetilde{\phi}$ itself. Using the information on $\mathrm{E}_{\mathrm{a}}$ one can interpolate with cubic splines using two endslopes given by $E_{a}{ }^{\prime}(\alpha, 0) \cong 0$ and $E_{a}{ }^{\prime}\left(\alpha, h_{1}\right) \cong\left(E_{a}\left(\alpha, h_{1}\right)-E_{a}\left(\alpha, h_{2}\right)\right) /\left(h_{1}-h_{2}\right)$. These endslopes are acceptable at $\mathrm{h}=0$ for any scheme with order larger than 1 . For the first order methods, in general, the slope at $\mathrm{h}=0$ is not zero. One could still obtain excellent results using the zero slope assumption for the first order methods. Once $E_{a}{ }^{(k)}(\alpha, 0)$ is obtained, one can calculate $a_{k}$ from Eq (A.5.8). As one might notice, $b_{1}$ is singular at $\mathrm{h}=0$ if $E_{a}{ }^{\prime}(\alpha, 0)=0$. In order to avoid this singularity, $E_{a}{ }^{\prime}(\alpha, \varepsilon)$ can be used to represent $E_{a}{ }^{\prime}(\alpha, 0)$ by using finite differencing at $h=\varepsilon$ where $\varepsilon$ is a small value. Having obtained $b_{0}, b_{1}$ and $b_{2}, \phi(0)$ can be calculated from Eq. (A.5.7) together with the definition (A.5.1) and (A.5.2). 


\section{Appendix B. The simplification of the particle equation}

In the current case, the equation of motion of a particle is

$$
\frac{d u_{P}}{d t}=F_{D}\left(u-u_{P}\right)+g_{x}\left(\rho_{P}-\rho\right) / \rho_{P}+F_{x}
$$

since $\rho_{P} \cong \rho$ the balance in Eq.(1) will be between inertial and drag, i.e.

$$
\frac{\partial u_{P}}{\partial t}=F_{D}\left(u-u_{P}\right)
$$

within $\Delta t$ assume $\mathrm{u}=$ const, so one obtains

$$
\frac{\partial\left(u_{P}-u\right)}{\partial t}=F_{D}\left(u-u_{P}\right)
$$

or

$$
\frac{u-u_{P}}{\tau}=F_{D}\left(u-u_{P}\right)
$$

which means the relaxation time $\tau$ is given by

$$
\tau=\frac{1}{F_{D}}=\frac{\rho_{P} d_{P}{ }^{2}}{18 \mu} \cong 10^{-8} \mathrm{sec}
$$

One can then conclude that these particles resume fluid velocity in a very short time, hence one can conclude that $u_{P}=u$. 
Appendix C. User guide for the cubic turbulence model in DREAM code

\section{C.1 Flow chart}

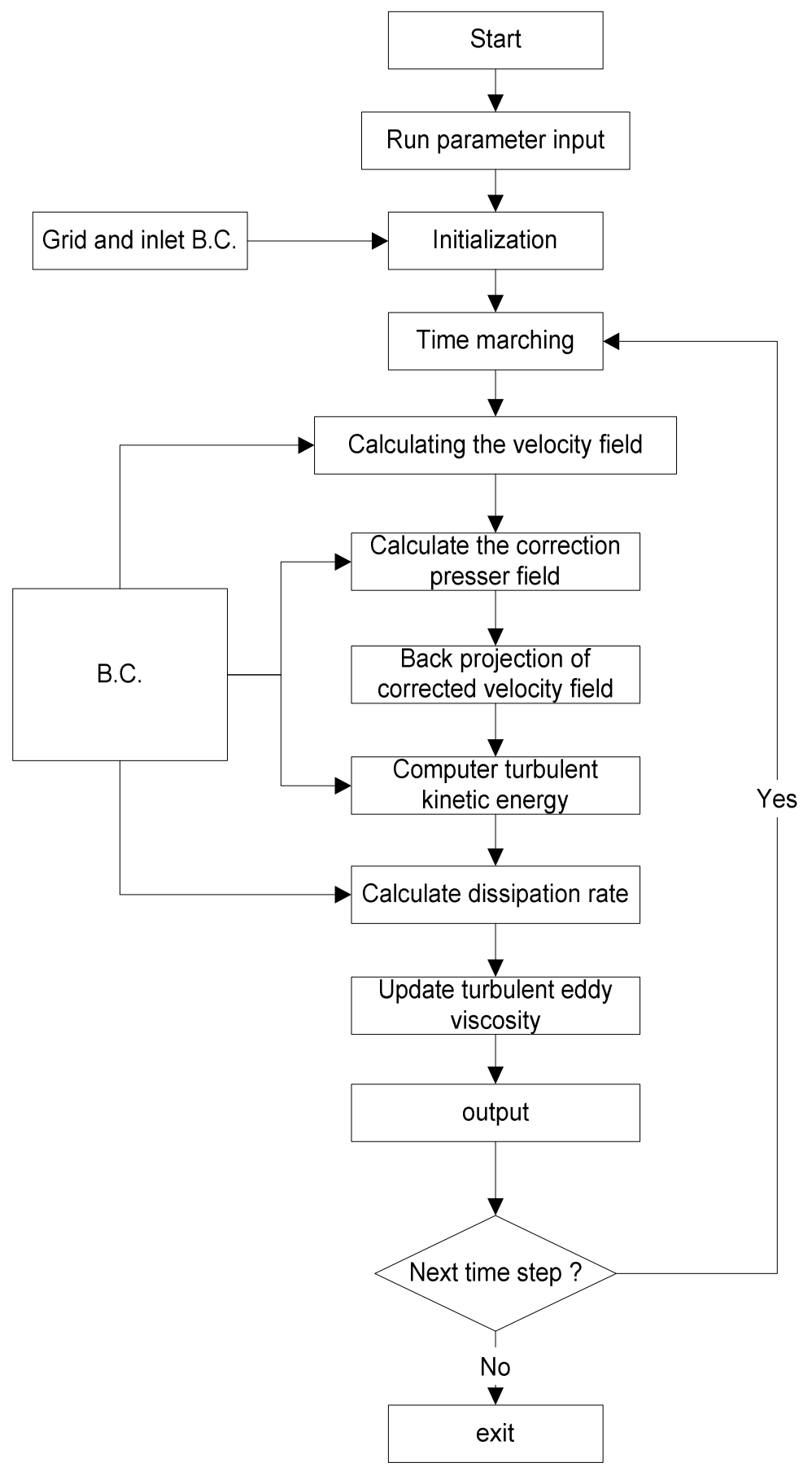

Figure C.1 The flow chart of DREAM with the cubic turbulence model 


\section{C.2 Description of subroutines for DREAM with the cubic turbulence model}

Bcond_ted.for: $\quad$ Sets the boundary conditions for $\varepsilon$

Bcond_tke,for Sets the boundary conditions for $\mathrm{k}$

Boundary.for Sets the boundary condition for different variables with different boundary types at different locations

Calc_uvel_impl.for: Calculates the u velocity component; boundary conditions are applied within the code

Calc_vvel_impl.for: Calculates the v velocity component; boundary conditions are applied within subroutine

Calc_wvel_impl.for: Calculates the w velocity component; boundary conditions are applied within subroutine

Calc_shear_stress.for Calculates Reynolds stresses for the whole domain

Calc_sor.for $\quad$ Calculates source terms for $\mathrm{k}$ and $\varepsilon$ equations

constants.for: All constants are defined in this location, including relaxation and implicitness factors for the solvers.

Dis_towall.for Calculates the shortest distance to wall

Dream.for: $\quad$ Main program; opens and closes most files; all subroutine calls are made from this program; correction of the velocities and application of boundary conditions is within this subroutine

fc_cblock.for: Defines all variables and allows for variables to be passes by defining only this common block, instead of individual variables.

fc_cparam.for: $\quad$ Sets the maximum grid that may be run on the computer.

fc_cpsolve.for: $\quad$ Sets up the common block parameters for the SIP3D, ICCG, and CGSTAB solvers

grid.for: Generate staggered grid in Cartesian coordinate system. Domain size is implemented in this location with units in meters 
initial.for: $\quad$ Sets up all initialization of velocity, $\mathrm{k}, \varepsilon$ and Reynolds stresses.

Inlet.for $\quad$ Read in or write out the inlet condictions

Interpolate.for: Interpolation scheme used for fully explicit schemes; User has option for central differencing, hybrid, upwinding first, third, and fifth order, central differencing scheme. Only written for uniform grids and must be modified for non-uniform grids

P_field.for: Calculates the pressure field through Poisson equation

Properties.for: Updates turbulent eddy viscosity

Print_utl.for output interested information

Psolvers.for provides three solvers for linear problems, ICCG: Incomplete Cholesky preconditioned Conjugate Gradient solver for symmetric matrices; CGSTAB solver; and SIP solver for 3D problems

Restart.for save and read information for restarting the program

Rhs_phi.for: The right hand side of each variable is computed using a general balance over a control volume and all field components may use this routine to calculate the r.h.s.

Scalar_implicit.for: calculates the scalar transport variable (i.e. k, $\varepsilon$, and temperature, etc.)

Shear_rot.for computers the shear and vorticity

Tur_model.for incorporates different turbulence models

Wall_functions.for: Calculates the $\mathrm{x}^{+}, \mathrm{y}^{+}, \mathrm{z}^{+}$wall units based on the law of the wall functions to determine $\mathrm{u}^{*}, \mathrm{v}^{*}, \mathrm{w}^{*}$ 